\title{
Directed Chaos in Magnetic Billiard Systems
}

\section{Dissertation}

zur Erlangung des Doktorgrades der Mathematisch-Naturwissenschaftlichen Fakultäten der Georg-August-Universität zu Göttingen

vorgelegt von

Manamohan Prusty

geboren in Dhenkanal (Orissa), Indien

Göttingen, 2006 
Referent :

Koreferent :

Tag der mündlichen Prüfung :
Prof. Dr. Theo Geisel

Prof. Dr. Kurt Schönhammer 


\section{To my best friend $\mathcal{E}$ my parents}





\section{Abstract}

\section{- - - English - - -}

In this thesis, we report our studies of various aspects of directed chaotic transport in the realm of quasi-one-dimensional billiard chains in the presence of a magnetic field perpendicular to the plane of the system. We treat all the particles (electrons) as classical objects without any kind of interaction effects. In particular we show that with proper parameters such systems can exhibit directed chaos even though the system is in equilibrium. For the first time we are able to apply a sum rule, originally presented in case of driven Hamiltonian systems (supporting directed chaos), and show that we can have an explicit expression for the average chaotic transport velocity inside the channel. We also show that the dispersion relation for our system is diffusive in nature and in certain circumstances we do observe long range effects, which can be diminished by proper adjustment in the boundary of the system. There has been a great amount of study in the field of conductance in nanowires based on the work of Landauer. This shows a relation between the conductance and the transmission characteristics of the system. Also with increase in the advancement of technological powers we are able to control the characteristics of the wire at the nano level. Thanks to these kind of applications, we show that the conductance in case of an infinite billiard chain with a single diffusive boundary (and the other is plane boundary supporting specular reflections), does not decrease to zero but rather goes to a finite constant. The other feature of the system is that the finite constant does not depend on the characteristic of the underlying chaotic dynamics, rather a novel construct of the regular phase space structure. This is in sharp contrast to Ohm's law and universal conductance fluctuations approaching zero in the limit of an infinite wire. Using a combination of two different probabilistic models we show that the conductance of the chaotic trajectories decreases exponentially as a function of length of the wire to this finite value. The consequence of the finite value is an effect of directed motion of chaotic trajectories. Finally we discuss the time-delay characteristics in case of a finite system and show that the behavior of directedness does show up in the distribution of transmission delay times of chaotic trajectories. However in the regular delay times we observe divergences going to infinite in number as the system approaches infinite. In contrast the delay time distribution of reflected trajectories shows universal power law behavior at long times. 


\section{- - - Deutsch - - -}

Diese Dissertation behandelt verschiedene Aspekte gerichteten chaotischen Transports im Kontext von quasi-eindimensionalen Billiardketten in Gegenwart eines senkrecht zur Ebene des Systems stehenden Magnetfelds. Alle Teilchen (Elektronen) werden als klassische Objekte ohne jegliche Wechselwirkung betrachtet. Im Besonderen wird gezeigt, dass mit geeigneten Parametern ein solches System gerichtetes Chaos aufweisen kann obwohl es sich im Gleichgewicht befindet. Wir konnten zum erstem Mal eine Summenregel anwenden, die ursprünglich in getriebenen Hamiltonschen Systemen benutzt wurde (gerichtetes Chaos unterstützend), und einen expliziten Ausdruck für die mittlere Chaotische Transportgeschwindigkeit im Kanal aufstellen. Außerdem wird gezeigt, dass die Dispersionsrelation für unser System diffusiver Natur ist und unter bestimmten Umständen langreichweitige Effekte zu beobachten sind, die durch geeignete Änderungrungen am Rand des Systems verhindert werden können. Es gibt eine Vielzahl von Untersuchungen auf dem Gebiet des Leitwerts in Nanodrähten basierend auf Arbeiten von Landauer. Dabei zeigt sich ein Zusammenhang zwischen dem Leitwert und den Transmissionseigenschaften des Systems. Mit zunehmendem technologischen Fortschritt wird man besser in der Lage sein, die Eigenschaften eines Drahtes auf nano-Ebene zu kontrollieren. Motiviert durch diese Anwendungen zeigen wir, dass der Leitwert im Falle einer unendlichen Billiardkette mit einer einzigen diffusiven Randbedingung (und ansonsten mit Spiegelreflexionen) nicht gegen Null geht, sondern gegen eine endliche Konstante. Eine Besonderheit des Systems ist, dass diese endliche Konstante nicht von den Eigenschaften der zugrundeliegenden chaotischen Dynamik abhängt, sondern ein neuartiges Konstrukt einer regulären Phasenraumstruktur darstellt. Dies steht in starkem Widerspruch zum Ohmschen Gesetz einerseits und auch zu universellen Leitwertfluktuationen, welche gegen Null gehen im Grenzübergang eines unendlich langen Drahtes. Unter Verwendung zweier verschiedener probabilistischer Modelle zeigen wir, dass der Leitwert der chaotischen Trajektorien als Funktion der Länge des Drahtes exponentiell auf einen endlichen Wert abfällt. Dieser endliche Wert ist eine Folge der gerichteten Bewegung chaotischer Trajektorien. Schließlich wird die Charakteristik der Verzögerungszeit im Falle eines endlichen Systems diskutiert und gezeigt, dass das gerichtete Verhalten chaotischer Trajektorien in der Verteilung der Verzögerungszeiten der Transmission auftritt. Hingegen divergieren die Verzögerungszeiten chaotischer Trajektorien im regulären Fall. Im Gegensatz dazu weist die Verteilung der Verzögerungszeit reflektierter Trajektorien universelles algebraisches Verhalten bei langen Zeiten auf. 


\section{Contents}

Abstract

Contents iii

List of Figures $\quad$ vii

List of Tables $\quad$ xxi

1 Introduction 1

1.1 Brownian Motion and Ratchets . . . . . . . . . . . . . . . . . . . . 1

1.2 Transport in Thermal Ratchets . . . . . . . . . . . . . . 3

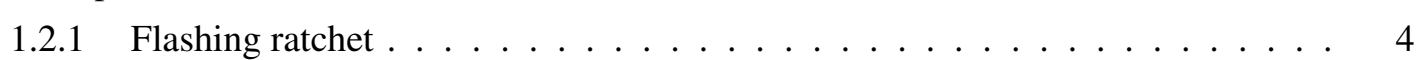

1.2.2 Parrondo's Games and Directedness . . . . . . . . . . . . . . . . 5

1.2.3 Parrondo's Games and Correlated Random Walk . . . . . . . . . . . . . . 7

1.3 Hamiltonian Ratchets . . . . . . . . . . . . . . . . . . . . 8

1.4 Transport Properties of Nanoelectric Structures $\ldots \ldots \ldots \ldots \ldots$

1.4.1 Ballistic Transport . . . . . . . . . . . . . . . . . . . . . . 11

1.4.2 Diffusive Transport . . . . . . . . . . . . . . . . . . . . . 13

1.5 Analysis and Synopsis . . . . . . . . . . . . . . . . . . . . . . . 14

2 Magnetic Billiard Chain with Periodic Structures 17

2.1 Introduction . . . . . . . . . . . . . . . . . . . . . . . . 17

2.2 Model of Periodic semi-circular Scatterers . . . . . . . . . . . . . . . . . . . 18

2.3 Phase Space Structure . . . . . . . . . . . . . . . . . . . . . . . 20

2.3 .1 Construction of Poincaré Map $\ldots \ldots \ldots \ldots \ldots \ldots$

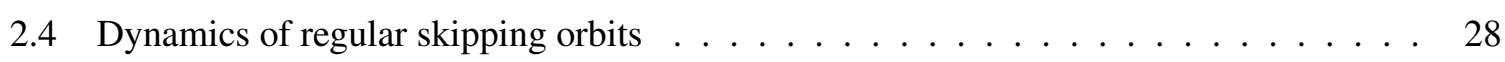

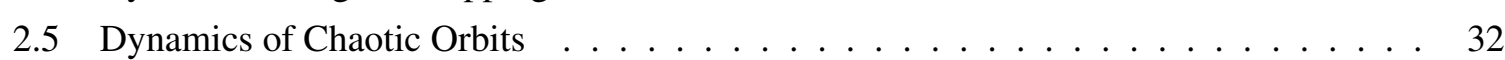

2.5 .1 Calculation of average chaotic transport velocity $\ldots \ldots \ldots 33$

2.5 .2 Dispersion of chaotic trajectories . . . . . . . . . . . . . . . . . 39 
2.6 Model with Convex Semi-circular Boundary . . . . . . . . . . . . . . . . . . . . . 43

2.6 .1 Average chaotic velocity . . . . . . . . . . . . . . . . . . . . . . . . . . . . 44

2.7 A comparison of the two models . . . . . . . . . . . . . . . . . . . 46

2.8 Summary and Conclusion . . . . . . . . . . . . . . . . . . . . . 50

3 Transport in a Magnetic Billiard Chain with a Rough Boundary 53

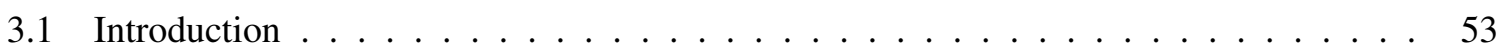

3.2 Billiard chain with a random corrugated surface $\ldots \ldots \ldots \ldots$. . . . . . . . 54

3.2.1 Average chaotic transport velocity . . . . . . . . . . . . . . . . . 58

3.2 .2 Diffusion Constant . . . . . . . . . . . . . . . . . . . . 60

3.3 Random Walk in One-Dimension . . . . . . . . . . . . . . . . . 63

3.4 Summary and Conclusion . . . . . . . . . . . . . . . . . . . . . . . 66

4 Transmission in a Finite Rough Magnetic Billiard 69

4.1 Introduction . . . . . . . . . . . . . . . . . . . . . . . . . 69

4.2 Conductance in a finite Wire . . . . . . . . . . . . . . . . 70

4.2.1 Transmission Probabilities based on Biased Diffusion . . . . . . . . . . . . . 74

4.2 .2 Persistent Random Walk . . . . . . . . . . . . . . . . . . . . . . . 77

4.3 Biased Persistent Random Walk (BPRW) . . . . . . . . . . . . . . . . . . . 78

4.4 Transfer Matrix Formalism for Transmission . . . . . . . . . . . . . . . . . . . . . 84

4.5 Ohm's Law: A FPE and PRW approach . . . . . . . . . . . . . . . . . . . . . 90

4.6 Recursive Formulation for Transmission . . . . . . . . . . . . . . . . . . . . . . 91

4.7 Summary and Discussion $\ldots \ldots \ldots \ldots \ldots \ldots 1 \ldots \ldots \ldots \ldots$

5 Delay Time Distribution $\quad 103$

5.1 Introduction . . . . . . . . . . . . . . . . . . . . . . . . 103

5.2 Connection between FPP and the probability density $\ldots \ldots \ldots$. . . . . . . 105

5.3 Distribution of delay times for the finite channel . . . . . . . . . . . . . . . . 107

5.3.1 Scaling of the Delay time distribution . . . . . . . . . . . . . . . . . 109

5.4 Distribution of Reflection Time Delays . . . . . . . . . . . . . . . . . . . . . . . . 112

5.5 Delay Time Distribution of the Regular Skipping Orbits . . . . . . . . . . . . . . . 115

5.5.1 Time Scales within the Distribution . . . . . . . . . . . . . . . . 116

5.5 .2 Special set of trajectories entering at $(0, b, \phi) \quad \ldots \ldots \ldots . \ldots . \ldots 119$

5.5 .3 General set of trajectories $\ldots \ldots \ldots \ldots$. . . . . . . . . . . . 122

5.5 .4 Occurrence of peaks in the probability distribution . . . . . . . . . . . . 127

5.6 Summary and Discussion . . . . . . . . . . . . . . . . . . . . . 130

6 Summary and Outlook 131 
Appendices

A Length of a section on the trajectory

B Jacobian of a transformation 139

C Calculation of $\langle\Delta x\rangle_{\phi}$ and $\langle\Delta l\rangle_{\phi}$

$\begin{array}{ll}\text { D Solution of the Biased Diffusion Equation } & 143\end{array}$

E Exit Probabilities for Biased Random Walk $\quad 145$

F Relation between components of scattering matrix and transfer matrix 149

G Transmission probabilities using the transfer matrix 151

$\begin{array}{ll}\text { H BPRW - A discrete approach } & 155\end{array}$

I Laplace transform solution for the FPP 161

$\begin{array}{ll}\text { References } & 165\end{array}$

$\begin{array}{ll}\text { Acknowledgments } & 171\end{array}$ 



\section{List of Figures}

1.1 Flashing Ratchet: A periodic asymmetric potential (piecewise linear saw-tooth potential) is switched on and off periodically in time. When the potential is off the Brownian particles diffuse and in the on state they settle down at the minimum of the potential. Due to the asymmetry in the potential larger number of Brownian particles get transported to a single direction (determined by the shape of the potential and the parameters of the model). This provides a net inflow of particle into a single direction. . . . . . . .

1.2 (a) The probability distribution of Game $\mathbf{A}$, as described in equation (1.1) and (b) The probability distribution of game $\mathbf{B}$, as in equation (1.2), using numerical simulations for the values of $\epsilon=0.1,0,-0.1$ (circle dashed, line and square dashed respectively). The data are obtained by simulating the game for $\eta=400$ times and the statistics is prepared with $10^{5}$ number of runs. For all the simulations, the initial capital is equal to zero at the beginning, and for each gain (loss) your capital is increased (decreased) by an amount equal to unity. . . . . . . . . . . . . . .

1.3 Numerical results of the combined game $\mathbf{A}$ and $\mathbf{B}$ when played randomly. The results shows that for small $\epsilon=0.001$, there is an overall increase of capital with the number of turns. The inset provides the probability distribution at the $400^{\text {th }}$ game, when played for $10^{5}$ number of runs. The simulations were obtained with an initial capital of amount zero. The vertical dashed line represents the average gain at the $400^{\text {th }}$ turn. . . . . . .

1.4 A particle at site $x$ coming from the previous site $x-1$ will have probability $p$ to transmit to site $x+1$ or else get reflected to site $x-1$ with probability $1-p$. This is called a correlated random walk. If the reflection rules are different for different direction of transport, then the correlated walk is biased. . . . . . . . . . .

1.5 Spatial distribution (a) of a continuous driven system, as in equation (1.5) showing the directedness of transport in a Hamiltonian ratchet. (c) shows the same kind of distribution in case of kicked Hamiltonian represented by equation (1.4). (b) and (d) shows the corresponding Poincaré sections of a unit shell with its embedded features. . 
1.6 Illustration of (a) Ballistic and (b) Diffusive regimes of transport. When the elastic mean path $l_{e}$ of the electron is larger than the system length $L$, the electron easily passes through the sample without any scattering providing a ballistic transport and if the elastic mean free path is smaller than the system length then the electron has to undergo multiple scatterings inside the sample providing a diffusive transport. . . . . . .

1.7 Sketch of the experimental setup for a transverse electron focusing. The electrons are injected through an emitter point contact $\mathbf{E}$ into the $\mathrm{Ag}$ crystal and focused into a point contact $\mathbf{C}$. Depending on the value of magnetic field the collector voltage will vary and we will observe peaks if the magnetic field satisfies equation 1.13$)$. . . . . . . . . .

1.8 Magnetic focusing of ballistic electrons in a Ag crystal. The peak (0) is caused by direct focusing into the collector, while the other peaks $(1,2, \ldots)$ are due to reflection from the boundary. (a) corresponds to the surface $\operatorname{Ag}(001), \mathbf{B} \|[100]$ and (b) results from $\operatorname{Ag}(011), \mathbf{B} \|[111]($ Reprinted with permission from [39]). . . . . . . . . . . .

2.1 The model of the magnetic billiard with periodic semi-circular scatterers of radius $R$. The displacement between the center of two nearby scatterers is $a$ and the width of the waveguide is $b$. A magnetic field perpendicular to the plane of the system distinguishes the movement of electrons from one direction (left to right) to the other (right to left) (for an example see figure $2.20 . \ldots \ldots \ldots \ldots$

2.2 In the presence of a magnetic field, perpendicular to the plane of the system, the trajectories of the electrons will consist of a special set of trajectories skipping (green curve) along the clean wall of the boundary whereas the motion along the other direction consists of trajectories hitting the semicircular obstacles (chaotic trajectories). As can be seen these chaotic trajectories (blue curve) contributes to the transport in the opposite direction of the regular skipping orbits. . . . . . . . . . . . . . .

2.3 Geometry showing the construction of Poincaré map. See details in the text. . . . . . .

2.4 Phase space portrait of the system shown in figure 2.1 $y$ and $\varphi$ are shown for $x=0$ $\bmod a$ and (a) $\cos \varphi>0$, (b) $\cos \varphi<0$. The data represent one chaotic and three skipping trajectories. The uniformity in the density of the chaotic region confirms the ergodicity property of the chaotic phase space. Pafameters used for the construction

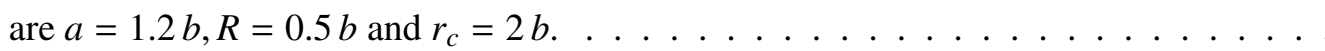


2.5 For high magnetic field $\left(r_{c}=0.7\right.$ for (a) and (b)) we can observe the occurrence of regular islands inside chaotic sea as in (a). Such type of periodic islands inside chaotic sea can occur due to periodic trajectories as shown in examples (e), (g) and (h). In particular, the black boundary near the border of the regular island in (a) corresponds to the Poincare map of the trajectory shown in (e). (c) and (d) (plotted for $r_{c}=0.6$ ) shows the Poincaré map of a typical chaotic trajectory (f) which keeps on moving in the direction of regular skipping orbits without being hit by the scatterer for a very long time. The occurrence of such islands and long range sticking orbits can significantly change the properties of electronic trapsport. The parameters used for (a)-(g) are $a=$ $1.2, b=1.0, R=0.5$. The cyclotron radius used for $(\mathrm{g})$ is $r_{c}=0.6$, while (h) have been plotted for $a=1.5, b=1.0, R=0.5, r_{d}=0.7$. The plot in (a) is for $\cos \varphi>0$ while the others $(b),(c)$ and $(d)$ are for $\cos \varphi<0 \ldots \ldots \ldots \ldots \ldots$

2.6 (a) Two transporting trajectories and (c) the corresponding Poincare map for the value of cyclotron radius $r_{c}=0.1$ and the scatterer radius $R=0.5$. The parameters used for the system are $a=1.2$ and $b=1$. The Poincare map in the negative direction of transport is for two regular skipping orbits. The empty looking regions, in the Poincaré map, are filled by pinned electrons as in (b). One regular skipping trajectory is seen in both panels of the Poincaré map. A regular skipping orbit will be seen in the positive panel of the phase space provided the center $\left(x_{c}, y_{c}\right)$ of the regular skipping orbit is such

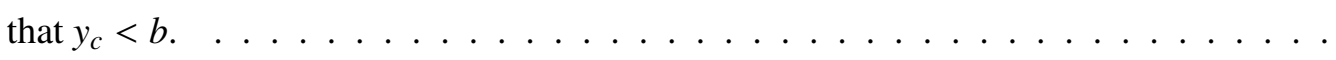

2.7 When the magnetic field is zero, for each trajectory moving in any direction there is always another trajectory moving in opposite direction, the result is a null average transport.

2.8 (a) The trajectory of an electron and (b) the corresponding Poincaré section when the magnetic field is very small. The value of cyclotron radius, $r_{c}=1000$ and other parameters of the system are same as in figure 2.6. This shows that almost all of the phase space are filled by a single chaotic trajectory leaving very little space for regular skipping drbits. Therefore, the average velocify of the chaotic trajectories will also be very

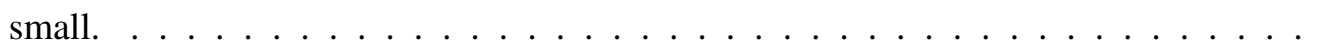

2.9 Variation of average velocity (for the parameters used in figure 2.8) for electrons starting with different initial conditions as a function of time. It shows that the value of average chaotic transport velodity (for almost all chaotic trajectories) remains close to zero for large cyclotron radius. . . . . . . . . . . . . . . . . .

2.10 The $y$-component of each arc's center of regularly skipping orbits is conserved through the entire length of the trajectory and the $x$-component of such trajectories will have a constant difference (longitudinal extension $\Delta x$ ) between any two nearby equivalent points on the trajectory. 
2.11 Calculation of time of travel for a section of arc in case of regular trajectories with nonzero average velocity. Since the speed $v_{0}$ is unity, the time for a section of the trajectory $=$ total length of the arc for the same section $(\Delta l) \ldots \ldots \ldots \ldots$

2.12 Velocity dependence of eight different regular skipping orbits as a function of time for different initial conditions shows a constant value depending on the initial position of the electron on the phase space. . . . . . . . . . . . . . . . . . . .

2.13 Velocity distribution of $N=5 \times 10^{5}$ skipping orbits equally distributed in phase space at different positions of time for the parameter values $b=1, a=1.2 b, r=2 b$ and $R=0.5 b$. For shorter time scales we can observe the appearance of peaks (dotted and dashed lines) in the distribution, while for very large times the distribution follows a smooth curve (dark line). . . . . . . . . . . . . . . . . . .

2.14 For the model of figure 2.1 and parameters of figure 2.4 the time averaged velocities of six different chaotic trajectories are shown as a function of time. For all the trajectories the average velocity approaches the analytical value predicted by equation (2.40) which is indicated by a horizontal line. . . . . . . . . . . . . . . . .

2.15 (a) Variation of average chaotic velocity (equation (2.40) as a function of its parameters namely cyclotron radius $r_{c}$ and the scatterer radius $R$, when $v_{0}=1, b=1$ and $a=1.2 b$. (b) The contour plot of the same function showing equi-velocity lines (larger values are shown with lighter shades)

2.16 (a) The dispersion of average chaotic velocities, for the model of figure $2.1, N=5 \times 10^{5}$ trajectories have been iterated for time $t=10^{3}$. The resulting distribution of average velocities is displayed as a histogram and compared to a Gaussian with same variance and mean (dotted line). The prediction of equation (2.40) for the average velocity is indicated by a vertical line. The numerical mean value of the distribution differs from that by $6.6 \times 10^{-5}$. (b) shows some of the higher moments of the distribution as a function of time. The straight lines indicate the corresponding moments $\langle(\bar{v}-$ $\left.\left.v_{\mathrm{ch}}\right)^{2}\right\rangle$ of a diffusively spreading Gaussian with $D=1.2$. Parameters are same as in the construction of figure $2.4 \ldots \ldots \ldots \ldots \ldots \ldots$

2.17 Characteristic large deviations arise from marginally stable periodic orbits. The full lines show two marginally periodic orbits. The dotted line is a non-periodic chaotic trajectory which remains in the vicinity of a marginally stable orbit for a long time. . .

2.18 Shown here the distribution of velocities for small obstacle radius of $R=0.01 b$ and no separation between any two consecutive semi-circular regions for the model of figure 2.1. The distribution show a Gaussian behavior. The dotted line show the corresponding Gaussian with same mean and variance. The analytical result of velocity from equation 2.40 ) is shown with a vertical dashed line . . . . . . . . . . . . . . . 
2.19 (a) A piece of chaotic trajectory is shown for $R=0.1 b$ and $a=2 R$. (b) One particular scattering of this trajectory from the lower boundary is magnified. Note that on this scale the curvature of the trajectory due to the magnetic field is hardly visible. (c) The normalized correlation between consecutive intersection angles of the segments with the line $y=R$ is shown with stars for the model of (a), with empty squares for $R=0.01 b$ and with black dots for the same $R$ but randomized $\xi_{n}$ (see text). The straight line shows the fitted $\operatorname{exponential} \exp (-1.65 n) \ldots \ldots \ldots$

2.20 Periodic semi-circular regions have been added to the upper boundary of a rectangular waveguide in construction of this model. This increases the total phase space volume available for an electron inside the channel. Parameters used for this model are $b=$ $1, a=1.2 b, R=0.5 b$ and $r_{c}=2 b . \ldots \ldots \ldots \ldots$

2.21 Phase space portrait for the parameters of figure 2.20 $y$ and $\varphi$ are shown for $x=0$ $\bmod a$ and (a) $\cos \varphi>0$, (b) $\cos \varphi<0$. The data represent five skipping and one chaotic trajectory. The uniformity in the density of the chaotic region confirms the ergodic property of the chaotic phase space. (c) The time averaged velocities of ten different chaotic trajectories is shown as a function of time. For all the trajectories the average velocity approaches the analytical value predicted by equation $(2.56)$ which is indicated by a horizontal line. . . . . . . . . . . . . . . . . .

2.22 The dispersion of average chaotic velocities, for the model of 2.20). $N=5 \times 10^{5}$ trajectories have been iterated for time $t=10^{3}$. The resulting distribution of average velocities is displayed as a histogram and compared to a Gaussian with same variance and mean (dotted line). The prediction of equation (2.56) for the average velocity is indicated by a vertical line. The numerical mean value of the distribution differs from that by $1.9 \times 10^{-5}$. Parameters are same as in the construction of figure $2.4 \ldots$. . .

2.23 Variation of average chaotic velocity (equation (2.56) with a negative sign) as a function of its parameters namely cyclotron radius $r_{c}$ and the scatterer radius $R$, when $v_{0}=$ $1, b=1$ and $a=1.2 b$. (b) The corresponding contour lines indicating equi-velocity lines (larger values are shown with lighter shades) $\ldots \ldots \ldots \ldots$. . . . . . . .

2.24 For small $R=0.01 b$, the dispersion of average chaotic velocities approaches a Gaussian with traces of large deviations for (a) figure 2.1 and (b) figure 2.20. $N=5 \times 10^{5}$ trajectories have been iterated for time $t=10^{3}$. The resulting distribution of average velocities is displayed as a histogram and compared to a Gaussian with same variance and mean (dotted line). The prediction of equations (2.40) and (2.56) for the average velocity is indicated by a vertical line in the respective distributions. Other parameters are same as in the construction of figure $2.20 \ldots \ldots \ldots \ldots$. . . . . . . . . 
2.25 (a) Variation of the variance $\sigma^{2}$ of the velocity distribution as a function of time for our models of (a) figure 2.1 and (b) figure 2.20. The parameters used are same as in the construction of figure 2.20. The plot show asymptotic decay in time as $1 / t$ with the co-efficient providing the Diffusion constant for the corresponding system. (b) The distribution of velocities for small obstacle radius of $R=0.01 b$ and no separation between any two consecutive semi-circular regions for the model of figure 2.20 shows that the distribution is indeed a Gaussian. The dotted line show the corresponding Gaussian with same mean and variance. The analytical result of velocity from equation $2.56)$ is shown with a vertical dashed line . . . . . . . . . . . . . . . .

3.1 The billiard model for directed chaos with two different kind of surfaces one which reflect the electrons specularly and at the other surface the reflections are essentially random (see text for details). Some typical electron trajectories (dashed line : regular, full line : chaotic) are shown when the lower wall is perfectly rough $(\alpha=0)$ such that the angles before and after a reflection are completely uncorrelated.

3.2 (a) Two trajectories (full line : chaotic, dashed line : regular) are shown for the model of figure 3.1. As the chaotic trajectory gets reflected from the rough wall of the channel it spans a horizontal distance $(\Delta x)$ during a time equal to the length of the corresponding section of the orbit $(\Delta l)$ (Since the the magnitude of velocity $v_{0}=1$ ). If $\phi$ denotes the initial angle of a trajectory at the lower wall of the channel then $\phi^{\prime}$ is the angle it will make th the upper wall of the channel. $\Delta x$ and $\Delta l$ for any section of a chaotic trajectory can be determined using these two variables ( $\phi$ and $\phi^{\prime}$ ) and the cyclotron radius $r_{c}$ (see text for details). Typical Poincaré maps for $x=0 \bmod 1$ when $r_{c}=b$ is shown for (b) $\cos \varphi>0$ and (c) $\cos \varphi<0$. A single chaotic trajectory fills both the regions of Poincaré map (shown here with dots) whereas the regular skipping orbits remain only to a single panel of the map (shown here like continuous lines in (c) for a set of them).

3.3 Chaotic phase space co-ordinate $(x, y, \varphi)$ can be represented in terms of $(x, \phi, l) . \phi$ is the inclination angle of the segment at the boundary and $l$ is the length of the point $(x, y, \varphi)$ from the reflection point at the boundary $\left(x_{0}, y_{0}=0\right)$. (See text for details of

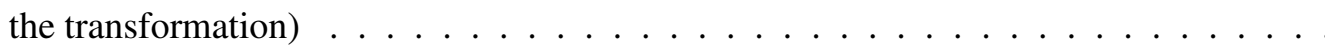

3.4 Dependence of the chaotic transport velocity (equation (3.20) $)$ and the diffusion constant (equation (3.29) ) on the cyclotron radius $r_{c}$ for random scattering at the lower wall $(\alpha=0)$.

3.5 Starting from the current segment (where $\left(\phi_{0}, l_{0}\right)$ is located), we can have $\mu$ identical segments before the current one and $v$ identical segments after the current one. Here this is shown for $\mu=2$ and $v=3$. The random reflections are represented by a black dot. The current point $\left(\phi_{0}, l_{0}\right)$ is shown diamond shaped. $\ldots \ldots \ldots \ldots$ 
3.6 Some higher moments of the distribution of $N=10^{4}$ time-averaged chaotic transport velocities in the magnetic billiard with a rough lower wall and $r_{c}=2 b$. The probability of specular reflection is (a) $\alpha=0$ and (b) $\alpha=3 / 4$. Straight lines indicate diffusive spreading with the diffusion constant given by equations $(3.30)$ and $(3.33)$. The latter correctly predicts that $D_{\text {ch }}$ is greater by a factor 7 in $(\mathrm{b}) \ldots \ldots \ldots \ldots$

3.7 The hopping process for the one-dimensional nearest neighbor random walk defined

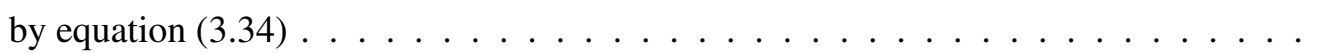

3.8 (a) The distribution of average velocities at various points of time $t=50,500$ and 1000 for the billiard chain with a rough boundary. The distribution very much agrees with the equation (3.41). The analytical value of average chaotic transport velocity as predicted from equation (3.20) has been shown with a vertical line. This predicted value nearly agrees with the statistical average value of $v_{\mathrm{ch}}=0.1695554$. (b) The small time distribution of velocity propagator at times $t=4$ (square), $6(\star), 8(+)$ and 10 (circle) for the billiard chain with a rough boundary. The analytical value of average chaotic transport velocity as predicted from equation (3.20) has been shown with a vertical line. For both of these $N=10^{7}$ number of phase space points have been chosen, with probability of inclination as in equation (3.1) along the line $y=0$ and $x \in(0,1)$, and propagated along the channel to find these distributions. . . . . . . . . . . . . .

3.9 The distribution of various moments (of velocities) for the billiard chain with a rough boundary obtained numerically (symbols) has been compared with the analytical prediction (straight line) provided in equation 3.42). Clear deviations are seen away from the power law behavior for smaller time scales. These deviations are attributed to correlations resulting from our distribution of particles. Nevertheless for larger time scales the results very much agree with the results of a Gaussian distribution. . . . . . . . .

4.1 In case of an electronic setup, the finite sized magnetic billiard (of length $L$ ) is coupled to two electron reservoirs placed on either side of the waveguide. The electron reservoirs can act either as a source (providing electrons for conduction) or sink (draining out electron from the waveguide) $\ldots \ldots \ldots \ldots$. . . . . . . . . 70

4.2 A 1D conductor connected to two reservoirs of chemical potentials $\mu_{1}$ and $\mu_{2}$. . . . . 71

4.3 (a) The total transmission $t(L)$ and $(b)$ the transmission $t_{\mathrm{ch}}^{\prime}(L)$ resulting from chaotic trajectories as a function of the system length for various values of the cyclotron radius. The figure $(a)$ shows that the total transmission approaches a constant value predicted by equation (4.13) (plotted with a horizontal line) and the chaotic transmission $t_{\mathrm{ch}}^{\prime}(L)$ decreases exponentially with the increase in system length. . . . . . . . . . . . . . .

4.4 The transmission probability $t_{\mathrm{ch}}^{\prime}(L)$ can be related to the exit probability $\pi_{L}^{\prime}(x)$ of a random walk starting at $x$ (inside the system) by a simple recursion relation (4.17). . . 
4.5 The variation of the Peclet length with the ratio of the cyclotron radius $r_{c}$ to the width of the channel $b$, when the magnetic field is not too strong $\left(r_{c} \geqslant 0.5 b\right) \ldots \ldots$

4.6 Comparison of numerical results for the chaotic transmission $t_{\mathrm{ch}}^{\prime}(L)$ (symbols) with the prediction of expression (4.25) (dashed line). The prediction was able to produce the length scale associated with the decay, but can not reproduce the correct pre-factor. . .

4.7 The asymmetric persistent walk (biased persistent random walk) takes place in 1D. A particle walks in the direction of motion of the previous step with probability $p^{+}$if it has arrived there from the left in the previous step and it reverses its direction with probability $q^{+}$. However if it has arrived from right then it continues to move in the left direction with probability $p^{-}$and reverses its movement with probability $q^{-} \ldots . .$.

4.8 The scattering model describing the asymmetric persistent walk (biased persistent random walk) (See figure 4.7 for details in 1D). . . . . . . . . . . . . . . . . . . . .

4.9 (a) The effective velocity in equation (4.45) as a function of the length of the waveguide remains a constant, whereas (b) the associated length scale $\Delta x$ given in equation (4.56) varies to a constan value. The vertical lines in (b) indicate the positions of twice the Peclet length $2 \lambda_{\mathrm{ch}} . \ldots \ldots \ldots \ldots \ldots \ldots$

4.10 In the framework of transfer matrix the total transfer matrix $T_{n}$ in $(a)$ can be broken up into $n$ equivalent transfer matrices $T$ as in $(b)$. Then the final probability $a_{n}=T_{n} a_{0}$ is related to $a_{0}$ with $n$ number of $T$ matrices as in equation (4.62) . . . . . . . . . . .

4.11 The final results for (a) total transmission (dashed line) from equation (4.76) is compared with the corresponding numerical results (in symbols) and ( $b$ ) the chaotic transmission (dashed line) from equation (4.75) is shown along with the numerical results (symbols). The predicted analytical results are in good agreement with the numerics even at small length scales. . . . . . . . . . . . . . . . . . . . .

4.12 For $(a)$ small and $(b)$ large values of cyclotron radius some of the trajectories will escape the system without being scattered within the channel. These type of systematic errors will show small deviations in the expected values of transmission probabilities. . . . .

4.13 Ratio of effective Peclet length $\tilde{\lambda}$ within the BPRW approach, predicted from equation (4.81), to the Peclet length of FPE approach $\lambda_{\mathrm{ch}}$. The ratio remains close to unity. The vertical lines show the position of $2 \lambda_{\mathrm{ch}}$ for a comparison with the figure 4.9 . . . . .

4.14 Scattering at a single impurity center. The particle entering from left will travel right with a transmission probability $t$, and will be reflected in the same direction with probability $r=1-t$. Similarly, the particle entering from right will be transmitted with probability $t^{\prime}$ and will be reflected with probability $r^{\prime}=1-t^{\prime} . \ldots . . . . .$. 
4.15 Scattering at two impurity centers. The particle entering from left/right might have to perform multiple maneuvers (multiple reflections) between the two centers before being transmitted/reflected in any of the directions. The total probability of transmission/reflection will be then the addition of all the possible paths leading to the final

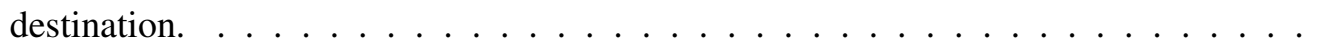

4.16 For a one loop transmission (path 1 (transmission $t$ ) $\rightarrow 2$ (reflection $r$ ) $\rightarrow 3$ (reflection $\left.r^{\prime}\right) \rightarrow 4$ (transmission $t$ ), denoted inside circles), the total contribution will be the multiplication of indiyidual probability contributions at each point of transmission/reflection. This provides a total contribution of $t r r^{\prime} t$ to one loop transmission. Then the total probability to get transmitted across the two sites will be the addition of many such loops. Similarly if the particle get reflected (path 1 (transmission $t) \rightarrow 2$ $($ reflection $r) \rightarrow 5$ (transmission $\left.t^{\prime}\right)$ ) the contribution to reflection will be $t r t^{\prime}$ and the total probability of reflection for two sites will be addition of several of these type of paths. (see text for details) . . . . . . . . . . . . . . . . .

4.17 (a) Scattering at three impurity centers. The particle entering from left/right will have to go through trajectories with fomplex patterns involving many orders of reflections between each sites with various degrees. Therefore, for simplification purpose, we replace the first two sites with an effective scatterer with appropriate reflection/transmission probabilities as shown in $(b) \ldots \ldots \ldots \ldots \ldots$

4.18 The results of the recursive formulation for the chaotic transmission $t_{n}^{\prime}(\times)$ are compared with the actual numerical data (lines) and the results of the transfer matrix formalism (in squares, from equations (4.64)) for scatterer segments of length (a) $L_{1}=1$ and (b) $L_{1}=5$. We observe good aqreement between the results of Recursive formalism and the transfer matrix formalisn, but they do not agree with the actual numerical results for small $L_{1}$. However the results nearly match each other with the numerical results for the value of $L_{1}=5$. This shows that there is a length scale associated with the scattering matrix formalism or the recursive formalism. . . . . . . . . . . .

4.19 Same as in figure 4.18, but only for $t_{n}$ when the length scale of the scatterer $L_{1}=1$. It shows the matching of transmission results with each other $(*$ :recursive, circle:transfer matrix and line:numerical data). The reason for this matching is that the asymptotic decay of $t_{n}$ to $\mu_{\text {reg }}$ completely takes over the discrepancies observed with the results of

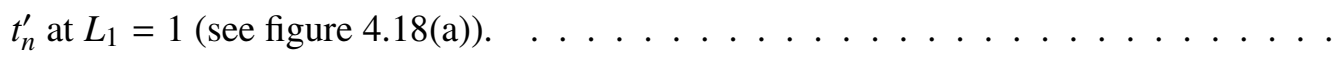

5.1 Diagrammatic relation between the occupational probability of a random walk (curved line) and the first passase probability (straight line). For the particle to be at $x$ at time $t$, it must reach $x$ at an earlier time $t^{\prime}$ and then return to the same position after an additional time of $t-t^{\prime} \ldots \ldots \ldots \ldots \ldots$. . . . . . . . . . . . 105 
5.2 For the electron entering at $x=0$ and exiting at $x=L$, the particle should pass through the intermediate point $x_{0}$ within the delay time and then exit from $x=x_{0}$ to $x=L$ within the first passage probability. This provides a recursion relation between the delay time distributions between the points $x=x_{0}$ and $x=L$ which is shown in equation (5.10). 108

5.3 The prediction of the equation (5.15) (plotted with lines) has been compared with the results of the transmission delay time distribution of the numerical simulation (plotted with symbols) obtain ed by simulating $N=10^{7}$ trajectories entering at $x=0$ equally distributed in the phase space i.e., with probability $P(\phi)=(1 / 2) \cos \phi,(\phi \in[-\pi / 2$ : $\pi / 2])$ and $P(y)=1,(y \in[0,1])$. The value of cyclotron radius used for the simulation is $r_{c}=4 b$ and $b=1 \ldots \ldots \ldots \ldots \ldots \ldots$

5.4 The results for the scaled distribution (5.18) (plotted using lines) for the transmission delay times has been compared with the results of the numerical simulation (in symbols). The conditions used for the numerical distribution are same as in figure (5.3). . . 110

5.5 The delay time distribution (symbols) has been plotted against an exponential fitted function (lines) as in equation 5.21). The results clearly shows the exponential nature of the delay time distribution for large values of $t$. The fitted exponential function agrees with numerical results especially for smaller values of $L$ and for $t>L / v_{\mathrm{ch}}$. . .

5.6 The distribution of reflection delay times for various lengths of the system $L=30,100$ and $L=1000$. It shows that the distribution is independent of the length of the system for large values of system length $L$. Inset shows that the distribution initially increases for a short while and then decreases. . . . . . . . . . . . . . . . . . . . . 112

5.7 The delay time distribution of reflected trajectories for $r_{c}=4$ (left) and $r_{c}=6$ (right) fitted against the function (5.26). The values of $\alpha, \beta$ and $\gamma$ are provided in table 5.2. The inset shows that the fitted function can not reproduce the results for very short times. 114

5.8 The delay time distribution of the regular skipping orbits show regular occurrence of divergences at some particular times marked by the vertical dashed line in (a) for the length of the system $L=20$. The number of these peaks gets increased in number as we go to higher lengths of the system size as shown in (b) for $L=200$. The distribution remains bounded within two the scales one equal to the length of the system and another $t_{c}$ provided by the average value of the critical value of velocity beyond which the trajectory becomes chaotic. The value of cyclotron radius chosen for these graphs is $r_{c}=4 b$, while the number of initial trajectories $N=10^{7}$ are equally distributed in the region of negative velocity of the Poincaré section. For clarity reasons we have discarded the chaotic tail in (a) . . . . . . . . . . . . . . . . . . . . 115 
5.9 For shorter system lengths, the distribution of delay times of electrons, moving from right to left of the channel, will show a chaotic tail. However the probability of chaotic trajectories getting transmitted against the drift decreases with the increase in the length of the system. The inset shows the distribution of only the regular skipping orbits (plotted in a different scale). As can be seen the distribution of regular skipping orbits is enclosed within two time scales $t_{0}$ and $t_{c}$ (see the text for details). The average chaotic time $t_{\mathrm{ch}}$ is shown with a vertical dotted line. . . . . . . . . . . . . . . 116

5.10 Any trajectory starting at any point $(x, y, \varphi)$ with $\varphi \in[\pi / 2,3 \pi / 2]$ to be a regular skipping orbit should follow the condition in equation (5.27). If the angle is such that $\varphi<\varphi_{\min }$ or $\varphi>\varphi_{\max }$, then the trajectory gets reflected randomly from the lower boundary making it a part of chaotic trajectories. . . . . . . . . . . . . . 117

5.11 A regular trajectory entering the channel at $(0, b, \phi)$ will eventually have to cross the entire length $L$ of the finite channel. Depending on the initial angle $\phi$, the trajectory can exactly fit into the entire length of the channel with an integer number of equal sections each of length $\Delta x$ (in which case the final section will have length zero) or it may not (leaving a remainder section of length $\delta x$ ). Accordingly the time will depend on $\Delta x$ and $\delta x$. See the text for details.

5.12 The center $\left(x_{c}, y_{c}\right)$ of the exit point and the beginning point for this last section of the trajectory of lateral distance $\delta x$ remains a constant. This provides a way for the calculation of the final angle $\varphi^{\prime}$ at the exit point. . . . . . . . . . . . . . 1

5.13 Variation of time $t(L, \phi)$ from equation $(5.42)$ as a function of the angular coordinate $\phi$ for the trajectories starting at the point $(0, b)$. The points marked with a $\times$ show the stationary points of the curve where the partial derivative of time $t(L, \phi)$ with respect to the angular coordinate $\phi$ vanishes. Because of this vanishing derivative the classical probability diverges at these points, providing peaks in the delay time distribution. . .

5.14 Any general regular electron entering the channel at $(0, y, \varphi)$ can be mapped to a point inside the reservoir $(x, b, \phi)$. Because of this mapping we extend the horizontal length of the trajectory by an amount $x$ into the reservoir. This simple construction helps to describe the dynamics in terms of the special set of trajectories (which are much more simpler). Nevertheless, we need to take care of this extra lateral displacement of $x$.

5.15 The variation of time $t(L, y, \varphi)$ as in equation (5.54) as a function $\varphi$ for various values of $y . y \simeq b$ corresponds to the outer curves and then it decreases to zero towards the inside. The critical average time $t_{c}$ is plotted with a dotted line and $\varphi_{\min }, \varphi_{\max }$ are the outer boundaries for the allowed values of $\varphi$ when $y \rightarrow b$. The different curves corresponding to different values of $y$ do not intersect each other. Th\&y initially decrease as $\varphi$ increases to $\pi$ and then increase with increasing the value of $\varphi \ldots \ldots \ldots 12$ 
5.16 The peaks in the distribution occur only when the derivative of $t(L, \phi)$ vanishes. These points corresponds to the end points of the strictures (thick black lines) on the higher values of $\varphi$ and reappearance of them on the lower value of $\varphi$. Moreover, the time derivative of the probability will follow a pattern as like these strictures. It is not clear if there is some correspondence between these strictures and the derivative of the probability distribution.

5.17 (a) If the lateral displacement $\Delta x$ is in commensuration with the length of the finite channel then all the points on the trajectory contribute to the same delay time (i.e., $\delta l_{1}-\delta l_{2}=0$ ), whereas if the trajectory is not in commensuration with the length of the channel, as in (b), then the points on the trajectory contribute to probabilities at different times $\left(\delta l_{1}-\delta l_{2} \neq 0\right)$. Therefore, in the former case we expect a peak in the distribution whereas in the latter case we may not expect one. . . . . . . . . . . .

5.18 The total time taken by the trajectory can be calculated using the addition of all the times taken by the electron for each individual sections of the trajectory before it comes out of the channel. . . . . . . . . . . . . . . . . . . . . . . . .

A.1 Section of a trajectory $(A O B)$ between two successive reflections from the lower boundary (points $A$ and $B$ ) is shown here with an intermediate point $O$ for $(a) \phi^{\prime}=0$ and (b) $\phi^{\prime} \neq 0 . \phi$ is the inclination angle of the arc with the lower boundary and $\phi^{\prime}$ is the inclination angle of the section of the arc at the upper wall of the channel. . . . . . . .

E.1 To calculate the exit probabilities, the particle starts its motion from $x=x_{0}$ at time $t=0$ and then moves to either of the directions. Depending on the direction of motion of the particle the exit probabilities will be different. . . . . . . . . . . . . . . .

F.1 Scattering model for BPRW describing transmission probabilities. The outgoing probabilities $\left(a_{+}, b_{-}\right)$relate themselves to the incoming ones $\left(a_{-}, b_{+}\right)$using a scattering matrix (equation (F.3) $)$. This can be modified to relate the right hand probabilities $\left(b_{-}, b_{+}\right)$ with the left hand probabilities $\left(a_{-}, a_{+}\right)$using a transfer matrix (equation $(\overline{F .60)})$. . . .

H.1 The persistent random walk and the phase space dynamics. $R$ is the fraction of regular trajectories which does not get scattered and move in a single direction (backward motion). $C$ is the fraction of chaotic trajectories out of which $f_{+}$is the fraction of chaotic particles which move in forward direction and $f_{-}$is the fraction of particles moving in the negative direction. The resultant bias of the chaotic trajectories is then given by $f_{+}-f_{-}$in stationary state. . . . . . . . . . . . . 157 
I.1 The solution of the random walker for a finite channel of length $L$. The random walker starts its movement from a point $x_{0}$ at time $t=0$ within the channel. On both sides of $x_{0}$ the solutions are different. In the positions greater than the value of $x_{0}$ the solution is given by $P_{>}(x . t)$ whereas for smaller values $x<x_{0}$ the solution is given by $P_{<}(x . t)$. The Laplace transform of currents to each ends are denoted by $J_{+}\left(x_{0}, s\right)$ for forward movement to $x=L$ and $J_{-}\left(x_{0}, s\right)$ for the backward movement to $x=0 \ldots \ldots \ldots$ 



\section{List of Tables}

5.1 Comparison of the values of $\mu$ obtained from expression (5.22) with the fitted value. . . 112

5.2 Values of $\alpha, \beta$ and $\gamma$ and the analytical value of $v^{2} /(2 D)$ for various values of cyclotron

radius. The table also provides an estimation for the value of $x_{0} \ldots \ldots \ldots$. . . . . . . 114 



\section{Chapter 1}

\section{Introduction}

"Why are things as they are and not otherwise?"

Johannes Kepler (1571-1630)

One of the important developments of the $20^{\text {th }}$ century was the developments in the semiconductor technology, which brought a revolution to the world, in the form of different devices (e.g., computers, digital cameras to name a few). This is due to the understanding of electronic transport in semiconductor devices. The initial development in research came from the Ohm's law characterizing the diffusive motion of electrons. Recently, the effect of non-diffusive properties has attracted a great deal of interest and has been studied in semiconductor nanostructures. In this thesis we focus on some of the aspects of this non-diffusive behavior, in the form of directed chaotic transport of electrons inside a billiardchannel in the presence of a perpendicular magnetic field. The idea of directed motion is not new, but has been first understood in case of ratchet systems. Below we describe the understanding of these systems.

\subsection{Brownian Motion and Ratchets}

In 1827, English botanist Robert Brown noticed that pollen grains suspended in water fluctuate about under the lens of the microscope. Others noticing the same phenomenon named it "Brownian motion" in honor of its discoverer. The phenomenon remained unexplained until Albert Einstein in 1905 decided to take a shot at a solution. What Einstein suggested was that the motion was due to the invisible water molecules hitting the visible particles and moving them a bit. Since the particles are hit continuously from all sides, they move in a random fashion. For a long time, life was good with this explanation, until Richard Feynman got his hands on the problem. Feynman suggested that we can build a tiny device that took advantage of Brownian motion [1]. The tiny device consists of a vane (on the right) and a ratcheted wheel (on the left) that is attached by an axel from which we can suspend 
something. The vanes are in a compartment filled with gas at temperature $T_{1}$, while the ratchet wheel is in another compartment of temperature $T_{2}$. Due to the bombardment of gas molecules on the vane, the vane oscillates and jiggles. Since the wheel at the other end of the axle turns only one way (because of the ratchet), motion in one direction will cause the axle to turn while motion in the other direction will not. Therefore, the wheel will turn slowly and may even be able to lift some weight. This was his idea of manufacturing a device out of the fluctuating motion of molecules.

Think what we could do if we can extract useful work from these vibrating molecules inside a system. We can recharge the batteries in our notebooks without ever having to plug it in and sell electricity to our neighbors. The possibilities are endless. But the question is if it can be done so? Sadly the answer is "it won't work". It's just another device violating the second law of thermodynamics and is also prohibited by statute. In the argument the temperature of the ratchet wheel does not play a role, therefore this machine would also work when $T_{2}=T_{1}$, which is a clear violation of the second law. This shows the weak point in this argument: the temperature of the ratchet wheel does play an important role. On the other hand if $T_{2}<T_{1}$, then the thermal motion of the pawl is of lower intensity, it gains the energy less often than the vanes does. As a consequence, the ratchet wheel rotates in the positive direction and is capable of lifting a body, if its mass is small enough.

It is here to note that, the presence of a ratchet introduces an essential asymmetry into the system. Therefore, at first sight, it appears that this asymmetry can prevent motion in one direction, while allowing the integration of momentum from molecular collisions, effectively rectifying thermal motion.

Feynman's ratchet device has a close connection to Maxwell's demon. In 1871, James Clerk Maxwell proposed a thought experiment. A wall separates two compartments filled with gas, and a little demon operates a door in the wall. It looks at oncoming gas molecules, and depending on their speeds it opens or closes the door, so that finally the fast molecules are collected in the right-hand compartment, and the slow molecules in the left-hand compartment. Because of the random kinetic energy of the molecules, the heat has been redistributed in such a way that useful work can be extracted from the system, which is again a violation of the second law of thermodynamics. The solution to this contradiction is that the demon itself is connected to the heat reservoir. A simple realization of this demon is a trapdoor on the right-hand side of the wall with a spring pushing it to the wall, so that the fast molecules can go across it from left to right, but the slow ones cannot. But this is just another form of the ratchet and pawl machine: the thermal motion of the spring opens and closes the door randomly, so the separation mechanism does not work after all.

However, Brownian motors do exist and work, although they do consume energy as they go. It turns out that if you start looking at the mechanisms by which DNA makes copies of itself [2] or the way proteins are folded as they are created, there is pretty convincing data to suggest that nature has engineered Brownian ratchets to assist in these fundamental biological processes [3, 4]. In real life many useful devices may utilize the ratchet mechanism, e.g., screwdriver, socket wrench, or a selfwinding pocket watch. The difference between Feynman's ratchet machine and the real life working 
machines is that the former has to be microscopic so that the thermal fluctuations can be effective, while the latter are macroscopic. As a consequence, the ratchet and pawl machine is exposed to equilibrium fluctuations, while working ratchet devices are driven by deterministic, non-equilibrium forces. The extra non-equilibrium forces, besides the ambient thermal fluctuations, make the microscopic mechanisms be able to do useful work. Therefore, Brownian ratchets may not be the means to generate free energy, but it may be core to understanding how we can extract useful work out of fluctuating systems. When molecules experience an asymmetry repeated along some generalized co-ordinate, but in a time-dependent, non-equilibrium fashion, they can generate a drift velocity by averaging over thermal noise $[5,6]$. The result is a Brownian molecular motor, i.e. a molecular machine that harvests thermally activated state-transitions to perform useful work. This mechanism has been used to explain the sophisticated operations of the molecular machines that transcribe genetic information [2].

A surge of interest in small scale systems (e.g., quantum dots, molecular motors, DNA transport etc) is to be found everywhere. This interest arises from the context: many of us find devices on the nanoscale and biological systems to be intriguing or fascinating. The enormous progress in the understanding of the nanoscale world has been fueled by developments in fields such as nanotechnology, protein folding, molecular motors, colloidal chemistry, to name but a few. In turn, the rapid development in these fields are made possible by the vastly improved ability to study and manipulate such systems, by the ever growing computational power to simulate their behavior accurately and by the parallel development of new theoretical tools. However the central interest have been based on the observation that on this small scale the effects of fluctuations become significant and even crucial. And, far from being a disturbing side-effect, it is now clear that noise may have an important or even an essential role in the operation of small-scale devices, leading to organized behavior that would not occur in the absence of noise. One example is the world of molecular motors [7], in which the energy from the random fluctuations is harnessed to produce directional motion of proteins. Other example include the transport properties of Brownian motors [6], DNA [4]. These examples are a part of the thermal ratchets, which uses the thermal fluctuations for uni-directional motion of the concerned Brownian particles.

\subsection{Transport in Thermal Ratchets}

If there is a thermal gradient in the Feynman's ratchet and pawl device $\left(T_{1} \neq T_{2}\right)$, then the device performs directed motion. However it is difficult to maintain large thermal differences over a small distance. Therefore, we need energy sources other than a thermal gradient to power a microscopic motor. If the system is spatially anisotropic, even if it is isothermal, Brownian motion can be biased in the presence of external non-equilibrium fluctuations $[6,8,9,10,11,12]$. The directedness of transport is due to a combination of diffusion and deterministic motion. It is also important that in such systems, the deterministic motion was due to the presence of an external time dependent periodic force whose 
time average is zero and the presence of thermal fluctuation. In general, the following principles are observed in most of the ratchet like systems: (i) Spatial periodicity and either invariance or periodicity under translations in time. (ii) All forces and gradients have to vanish after averaging over space, over time and over statistical ensembles. (iii) The system has to be driven out of thermal equilibrium and there should be no symmetries prohibiting a ratchet effect and (iv) There needs to be a finite amount of thermal noise inside the system. Let us consider few simple systems to show how they work.

\subsubsection{Flashing ratchet}

Consider an overdamped Brownian particle moving along a 1D asymmetric potential. The ratchet potential is asymmetric and periodic in space (see an example in figure 1.1) and is switched on and off periodically in time. This on and off mechanism of the potential introduces a non equilibrium

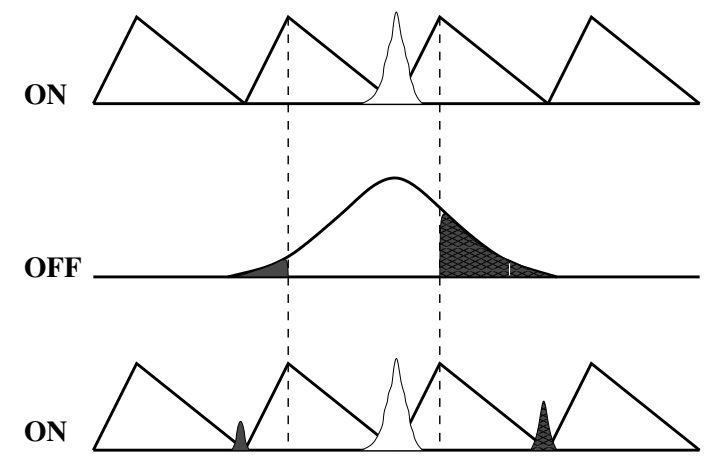

Figure 1.1: Flashing Ratchet: A periodic asymmetric potential (piecewise linear saw-tooth potential) is switched on and off periodically in time. When the potential is off the Brownian particles diffuse and in the on state they settle down at the minimum of the potential. Due to the asymmetry in the potential larger number of Brownian particles get transported to a single direction (determined by the shape of the potential and the parameters of the model). This provides a net inflow of particle into a single direction.

fluctuation and therefore contributes to the transport. Because of the on and off mechanism of the potential, this is called a flashing ratchet. In figure 1.1, we have shown a piecewise linear 'saw-tooth' potential acting as a ratchet. The diffusing particle starts its motion at $x=0$ in the off state. After time $T$, corresponding to the periodicity of the "flashing", a significant portion of probability gets beyond the top of the righthand tooth when switched on, while a negligible amount gets over the left hand part. Because of dissipation the Brownian particles will settle down into one of the bottom points in the potential. Thus, the Brownian particles can be transported in the right hand direction due to the asymmetry of the ratchet potential, provided the fluctuation frequency is properly chosen. In contrast if the parameters of the system are changed properly, then the direction of the particle can be reversed $[13,14,15,16]$. This simple example suggests that the directionality for the motion of a Brownian 
particle can be achieved.

\subsubsection{Parrondo's Games and Directedness}

Parrondo's games, devised by J. M. R. Parrondo, are inspired by Brownian ratchets. It consists of two games $\mathbf{A}$ and $\mathbf{B}$ each one of them can be fair i.e., each of them has the probability of winning $P(W)=$ probability of losing $P(L)=1 / 2$, or can be losing $P(W)=1 / 2-\epsilon$. On win, a player will be able to gain $€ 1$, or else the player loss $€ 1$ from his/her capital. However if both the games are played randomly then the outcome of the game may behave in an unexpected way. Instead of losing the game it can happen that the capital will go on increasing. Game A can be thought of tossing a weighted coin (or going on a biased random walk) [17]

$$
\text { Game A : }\left\{\begin{array}{l}
P(W)=\frac{1}{2}-\epsilon, \\
P(L)=\frac{1}{2}+\epsilon
\end{array} .\right.
$$

For $\epsilon=0$ the game is fair; and numerical simulation of the game shows that the probability curve is a Binomial distribution (see figure 1.2(a)). The figure also confirms that the game is losing for $\epsilon>0$ and fair for $\epsilon=0$. However game $\mathbf{B}$ can be thought of as a combination of two games as it is played with two biased coins, a 'bad coin' with probability of winning $p_{0}=1 / 10-\epsilon$ and a 'good coin' with probability of winning $p_{1}=3 / 4-\epsilon$. The player tosses the bad coin if his/her capital is a multiple of 3
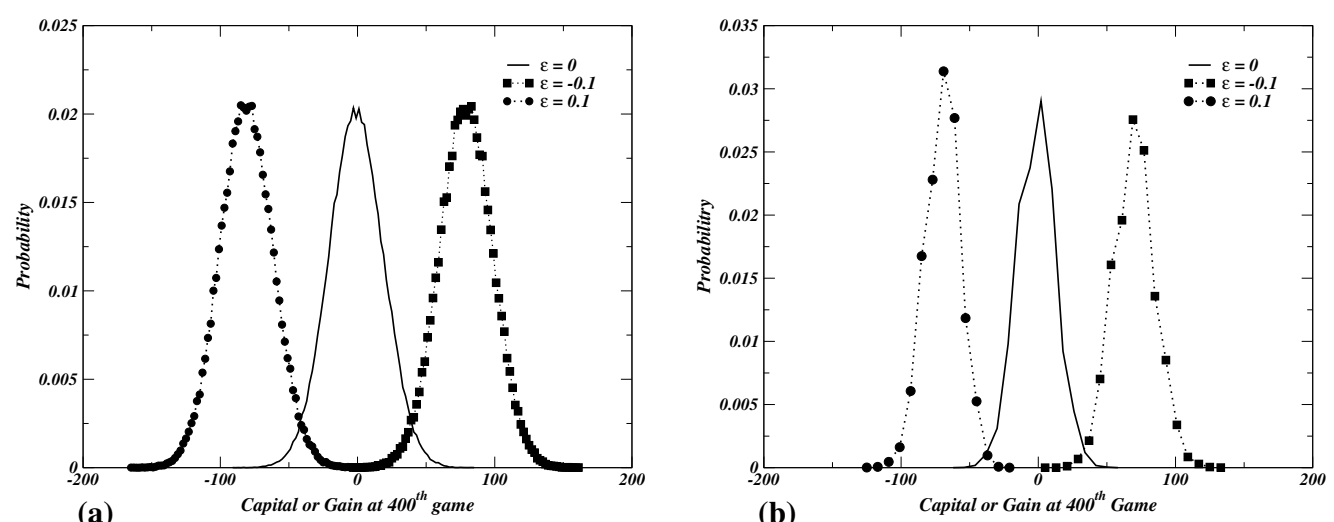

Figure 1.2: (a) The probability distribution of Game $\mathbf{A}$, as described in equation 1.1 and (b) The probability distribution of game $\mathbf{B}$, as in equation (1.2), using numerical simulations for the values of $\epsilon=0.1,0,-0.1$ (circle dashed, line and square dashed respectively). The data are obtained by simulating the game for $n=400$ times and the statistics is prepared with $10^{5}$ number of runs. For all the simulations, the initial capital is equal to zero at the beginning, and for each gain (loss) your capital is increased (decreased) by an amount equal to unity. 
otherwise the good coin is tossed.

$$
\text { Game B : }\left\{\begin{array} { l l } 
{ \text { capital } \quad \operatorname { m o d } 3 = 0 } \\
{ \text { capital } \quad \operatorname { m o d } 3 \neq 0 }
\end{array} \left\{\begin{array}{l}
P(W)=p_{0} \\
P(L)=1-p_{0} \\
P(W)=p_{1} \\
P(L)=1-p_{1}
\end{array} .\right.\right.
$$

Numerical simulation of this game is shown in figure 1.2(b). The simulation shows that for $\epsilon=0$ the game is fair and for positive values of $\epsilon$ there is a net loss of the player.

The paradox arises when the game $\mathbf{A}$ comes into play along with game $\mathbf{B}$. Since the game $\mathbf{A}$ is played with the same coin independent of the amount of capital it drives the probability to a uniform distribution. Although the game A consists of a negative trend of losing, it increases the probability of using the good coin for game $\mathbf{B}$. The result is an overall positive trend enough to make a win. Numerical simulation (shown in figure 1.3) of the combined game played randomly confirms this results. .

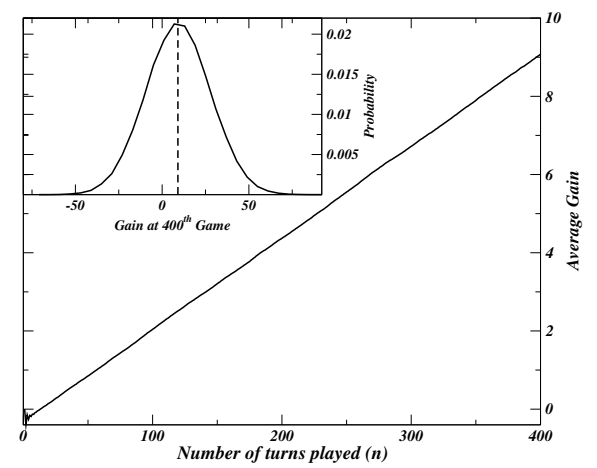

Figure 1.3: Numerical results of the combined game $\mathbf{A}$ and $\mathbf{B}$ when played randomly. The results shows that for small $\epsilon=0.001$, there is an overall increase of capital with the number of turns. The inset provides the probability distribution at the $400^{\text {th }}$ game, when played for $10^{5}$ number of runs. The simulations were obtained with an initial capital of amount zero. The vertical dashed line represents the average gain at the $400^{\text {th }}$ turn.

The connection between the rectification in the game and the ratchets will be clear if we think game $\mathbf{A}$ as a Brownian particle undergoing diffusion (when $\epsilon=0$ ), and game $\mathbf{B}$ acting as a ratchet potential. When we use the bad coin in game $\mathbf{B}$ the probability of winning is very low whereas when we use the good coin the most likely move will be gaining $€ 1$. Also the probability of using the bad coin is very small (1/3) compared to the use of good coin (2/3). Therefore it can be compared to a saw-tooth ratchet potential (see figure 1.1) with a short spatial interval when the force is negative and a longer interval when the force is positive. We already have discussed that the on-off mechanism of the flashing ratchet directs the diffusive particles to a single direction. Thus, on random playing, the ratchet potential of game $\mathbf{B}$ rectifies the fluctuations of game $\mathbf{A}$. Thus, there is an interplay between the fluctuation and the 
ratchet potential which rectifies the current for the particle.

\subsubsection{Parrondo's Games and Correlated Random Walk}

In the the coin flipping game of Parrondo the probability of winning on each play depends on the outcome of the previous play. Therefore, we need to add an extra bit of information of the previous step. In other words it can be described by using a correlated random walk. Figure 1.4 illustrates this model. The figure describes the movement of a particle based on the movement in the previous step.

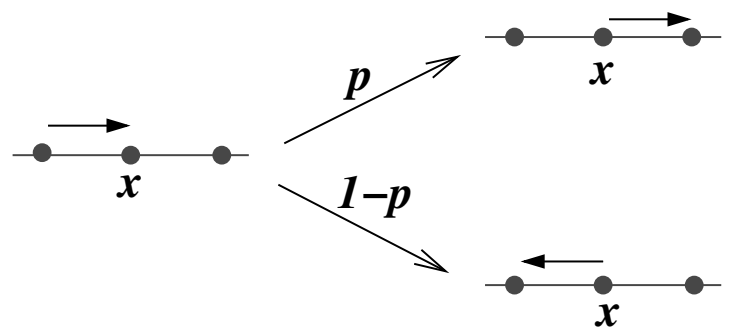

Figure 1.4: A particle at site $x$ coming from the previous site $x-1$ will have probability $p$ to transmit to site $x+1$ or else get reflected to site $x-1$ with probability $1-p$. This is called a correlated random walk. If the reflection rules are different for different direction of transport, then the correlated walk is biased.

The arrow indicates the direction of motion of the particle. It says that the particle has probability $p$ of continuing its motion in the direction it came, as in the previous step; and $1-p$ is the probability of reversing its motion in the next step. For $p=1 / 2$, the motion is uncorrelated and it gets back to the standard random walk. However if $p \neq 1 / 2$, then the probabilities for successive steps are dependent on each other. To get an biased correlated random walk, the probabilities should not only depend on the previous step but it needs to break the symmetry under parity. Based on its history Parrondo devised history based games [18] independent of the amount of capital which show up the paradox of winning the game even if the individual games are losing.

Apart from these examples of directed motion there are a lot more other kind of ratchet models e.g., vibrational ratchets, rocking ratchets and many more which show directed motion. See the Ref. [13] for a variety of examples. The transport of an overdamped particle, in all these thermal ratchets, can be described using a periodic potential breaking time reversal symmetry and subject to a driving force in the presence of thermal noise. The important features of all these ratchet models is that they incorporate noise (or some variants of it) in order to show directed transport. In contrast we can have ratchets in absence of any thermal noise. Such kind of ratchets are called Deterministic Ratchets. Important among these ratchets are the "Hamiltonian Ratchets" which conserve the total energy of the system. Below we discuss some of the features and implications for these models. 


\subsection{Hamiltonian Ratchets}

If the deterministic ratchet have a tendency to behave conservatively without any dissipation, they can be associated with Hamiltonian Ratchets. The salient feature of such ratchet systems in comparison with a dissipative system is the time-inversion invariance provided the time-periodic driving is symmetric. Another basic feature is the generic appearance of chaos. As a result, the behavior of the system depends in general on the initial conditions. Therefore such kind of systems can exhibit all types of behavior starting from diffusion [19] to Lev́y flights [20, 21, 22, 23, 24] and directed transport [25, 26, 27]. According to Curie's principle, if the spatial periodic potential $V(x)$ and the time $\tau$ periodic driving $y(t)$ are symmetric,

$$
\begin{gathered}
V(x)=V(2 L-x) \\
y(t+\tau / 2)=-y(t)
\end{gathered}
$$

then such a system cannot support a directed motion. In contrary, breaking of both the symmetries can support transport in Hamiltonian ratchets [28], and even breaking of one of these symmetries is generally sufficient to ensure a net motion of the particle [29]. To get an idea of transport in such deterministic systems let's consider the kicked Hamiltonian [28, 30]

$$
H(x, t)=\frac{p^{2}}{2}+\frac{K}{2 \pi} \sum_{n} \delta(t-n) .
$$

This system shows a robust directed behavior (see figure 1.5(c)). However in other examples as in [28]

$$
H(x, p, t)=\frac{p^{2}}{2}+V(x, t)
$$

with

$$
\frac{\partial V(x, t)}{\partial x}=\left(\frac{2 \pi}{\omega}\right)^{2}[\cos (2 \pi x)+0.6 \cos (4 \pi x+0.4)-2.3 \sin (2 \pi t)-1.38 \sin (4 \pi t+0.7)] .
$$

the peak is not so much pronounced but nevertheless it does show directedness of the chaotic trajectories (see figure $1.5(a)$ ).

Hamiltonian ratchets show directedness on the basis of broken spatio-temporal symmetries. But on the basis of the features of the phase space it has been shown that an embedded phase space with chaotic and regular properties can be enough to have directedness of chaotic trajectories. Based on the compactness of the phase space structure a sum rule [28] was presented providing a relation between the chaotic transport velocity of the chaotic sea and the corresponding regular counterparts. Nevertheless all such systems are rather explicitly depend on "time". The question arises if that can be a factor for 

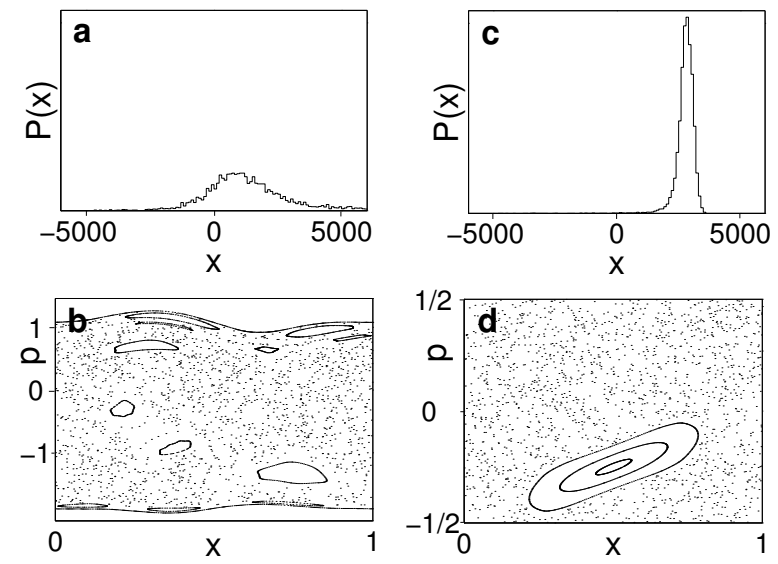

Figure 1.5: Spatial distribution (a) of a continuous driven system, as in equation (1.5) showing the directedness of transport in a Hamiltonian ratchet. (c) shows the same kind of distribution in case of kicked Hamiltonian represented by equation (1.4). (b) and (d) shows the corresponding Poincaré sections of a unit shell with its embedded features.

driving the system away from equilibrium and bring in the directedness of the chaotic trajectories? The answer lies within the realm of the underlying asymmetries in the phase space. Thus, only a symmetric phase space cannot show the property of directedness. Therefore, in principle a magnetic field, which can break the symmetry of the trajectories, can support the directionality of chaotic trajectories. The application of magnetic field lies in condensed matter physics and there is a vast amount of literature available for the study of resistances in Hall effects. So, we can rely on such kind of setups in order to show the directedness of chaotic trajectories. These kind of ballistic systems, with no disorders inside the system, can be modeled using the billiard models. The great advantage of a billiard system is that they themselves does not have any kind of potential within the system but are represented by specular or disorder reflections at the boundary. This makes such systems simple to study all kind of effects. In the second chapter and the chapters to follow we will see that the presence of a magnetic field in billiard chains having broken symmetry in the phase space indeed supports directedness of chaotic trajectories and based on the principles of sum rule we can explicitly calculate the average chaotic transport velocity on the basis of system parameters (so far all the analysis on the ratchets are based on numerical figures).

The applications of ratchet mechanism is wide ranged and the research includes its application in the areas of quantum dots [31], Josephson-junctions [32, 33, 34], optical lattice [35, 36], superconductors [37] and many more including the original idea to biological systems. Recently there have been a lot of study in the field of nanotechnology (starting from quantum dots to carbon nanotubes to molecular devices) because of their potential applications. So let us divert a bit from the directed motion to 
the field of mesoscopy and discuss some of the transport properties inside them.

\subsection{Transport Properties of Nanoelectric Structures}

Modern fabrication techniques (lithography, implantation, etching etc) allows to prepare structures which are significantly smaller than a micrometer from a bigger sample (e.g., an array of quantum dots), or by using smaller pieces to prepare a bigger one (e.g., carbon nanotubes, molecular wires). However one finds that the transport and many other effects are more or less common in all the materials. What counts, in these nanomaterial structures, are the intrinsic properties and different length scales associated with the carrier (electrons).

At zero temperature the electrons becomes stacked (due to Pauli's exclusion principle each electron occupies a given energy level) in energy to a maximum value called the Fermi Energy $E_{F}$. Its value depends on the concentration of the conduction electrons. The wavelength associated with this energy

$$
\lambda_{F}=\frac{2 \pi \hbar}{\sqrt{2 m^{*} E_{F}}}
$$

is called the 'Fermi wavelength' of the electron. In the above equation $m^{*}$ is the effective mass of the electron inside the sample.

However the conductor is not always a perfect conductor. There can be irregularities or impurities inside it. As a result the electron, as they travel, can get scattered from these centers unevenly. The 'mean free path' is a measure for the average distance between two such scattering events. If the scattering event is elastic then it is called elastic mean free path, or if it is inelastic then it is called inelastic mean free path. The elastic mean free path $l_{e}$ can be computed from the electron mobility $\mu_{e}$ (which is a measure of drift velocity in presence of an electric field) by using the equation

$$
l_{e}=\tau_{e} v_{F}=\frac{m^{*} \mu_{e}}{e} v_{F} .
$$

where $\tau_{e}$ is called the elastic scattering time.

As the electron get scattered from inside the conductor its phase get altered. Depending on the type of scattering event the change in phase can be different. In particular if the scattering is elastic with a static scattering center, then the phase is shifted by the same amount if the electron will travel the same path a second time. Therefore, in elastic scattering events, the phase of the electron is usually not randomized. In contrast, in inelastic scattering events the phase shift of the electron is different each time it gets scattered. Depending on the length of the sample this phase can influence the type of transport inside the conductor.

Moreover, In the presence of a magnetic field $B$ the electrons get deflected by the Lorenz force. Therefore, the free electrons will travel along a circular orbit whose radius, the cyclotron radius $r_{c}$, can 

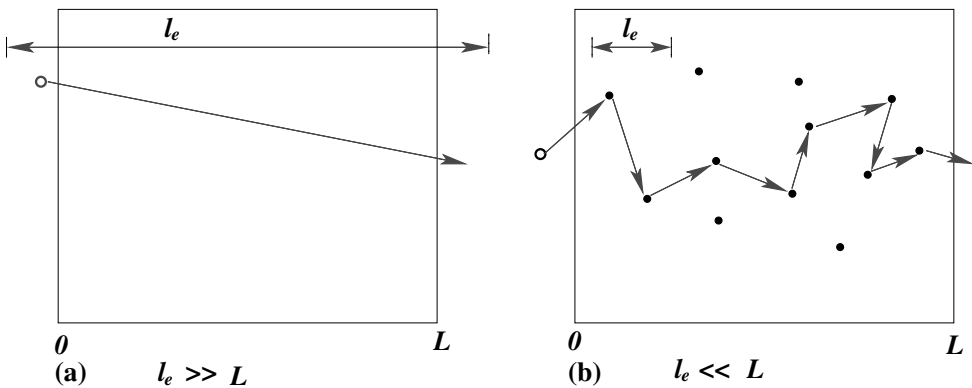

Figure 1.6: Illustration of (a) Ballistic and (b) Diffusive regimes of transport. When the elastic mean path $l_{e}$ of the electron is larger than the system length $L$, the electron easily passes through the sample without any scattering providing a ballistic transport and if the elastic mean free path is smaller than the system length then the electron has to undergo multiple scatterings inside the sample providing a diffusive transport.

significantly change the transport properties of the system (especially if $r_{c}$ approaches the dimension of the sample). The cyclotron radius can be calculated from the balance of the centrifugal force and the Lorenz force

$$
e v_{F} B=\frac{m^{*} v_{F}^{2}}{r_{c}} \Rightarrow r_{c}=\frac{m^{*} v_{F}}{e B}
$$

Using these length scales of the electron and the system length $L$ we can have different transport regimes inside the sample. In case the elastic mean free path is larger than the system length, the electron traverse the system without any scattering. Such a regime is called ballistic (see figure 1.6(a)). Depending on the magnitude of the Fermi wavelength $\lambda_{F}$ in comparison to the sample length it can be either classical ballistic (when $\lambda_{F}<L$ ) or quantum ballistic $\left(\lambda_{F}>L\right.$ ). However, if the elastic mean free path is smaller than the sample length many scattering events occur inside the sample. Therefore, the carriers will travel randomly inside the system. This regime is called diffusive (see figure 1.6(b)). But if the phase coherence length $l \varphi$ is larger than the system length $L\left(l_{\varphi}>L\right)$ then quantum effects, due to the wave nature of electron, are expected to play a role in the transport of the electron. This diffusive regime is called quantum diffusive regime.

\subsubsection{Ballistic Transport}

As discussed, if the elastic mean free path of the carrier electrons exceed the sample length of the system then ballistic transport can be observed in the sample. In order to study the transport properties of a mesoscopic nanostructure a current is flown through two contacts while the response is measured by two voltage probes. The ratio between the voltage and the current provides the bulk resistance of 
the sample. If the dimensions of the conductor is large the conductance is given by Ohm's Law [38]

$$
G=\sigma \frac{W}{L}
$$

where $\sigma$ is the conductivity which is a material parameter independent of the sample dimensions, $W$ is the width and $L$ is the length of the conductor. If this Ohmic scaling is to hold as the sample length is reduced, then the conductance should grow indefinitely. However, experimentally the conductance goes to a limiting value, if $l_{e} \ll L$. This resistance arises from the interface between the contacts and the conductor (which are very dissimilar materials). Therefore, this resistance is referred to as the contact resistance. Moreover the conductance depends on the number of transverse modes $N$ and goes down with discrete steps. The Landauer formula [38] provides the conductance as

$$
G=\frac{2 e^{2}}{h} N T
$$

where $T$ represents the average probability that an electron injected at one end gets transmitted to the other end.

\section{Magnetic Focusing}

In presence of a perpendicular magnetic field, a ballistic conductor shows that at particular magnetic fields the electrons can be focused into the collector region of the sample. In figure 1.7, we have shown the experimental setup for a beam of ballistic electrons in a Ag crystal [39]. On application of a

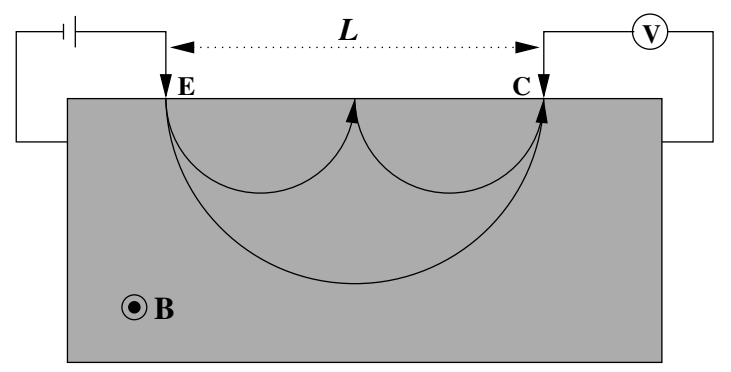

Figure 1.7: Sketch of the experimental setup for a transverse electron focusing. The electrons are injected through an emitter point contact $\mathbf{E}$ into the $\mathrm{Ag}$ crystal and focused into a point contact $\mathbf{C}$. Depending on the value of magnetic field the collector voltage will vary and we will observe peaks if the magnetic field satisfies equation (1.13).

magnetic field the electrons from the emitter $E$ gets deflected due to Lorenz force. If the magnetic field is such that the cyclotron radius $r_{c}$ satisfies the condition

$$
2 p r_{c}=L, \quad(p=1,2,3, \ldots)
$$




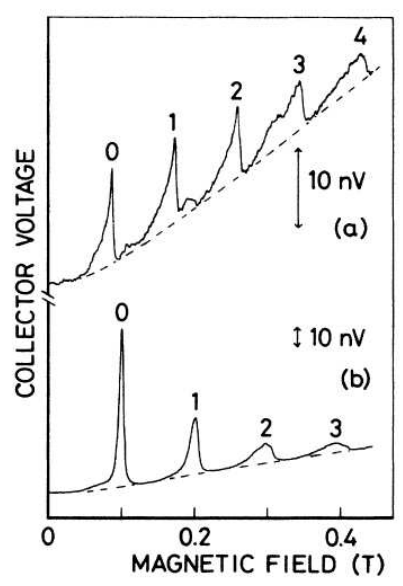

Figure 1.8: Magnetic focusing of ballistic electrons in a Ag crystal. The peak (0) is caused by direct focusing into the collector, while the other peaks $(1,2, \ldots)$ are due to reflection from the boundary. (a) corresponds to the surface $\operatorname{Ag}(001), \mathbf{B} \|[100]$ and (b) results from $\operatorname{Ag}(011), \mathbf{B} \|[111]$ (Reprinted with permission from [39]).

or in terms of the magnetic field

$$
B=\frac{2 m^{*} v_{F}}{e L} p, \quad(p=1,2,3, \ldots)
$$

then the electrons get focused to the collector $C$ with number of reflections given by $p-1$. The measured voltage at the collector contact is shown in figure 1.8. The peaks occur at magnetic field values given by equation (1.13). Now instead of varying the magnetic field $B$, if we think the point source at $E$ is such that it is providing the electrons within an angle $\varphi$, the angle being measured from the positive $x$-axis (along the length of the conductor), then we can expect the voltage to be peaking at those angles for which the electrons are collimated at the collector. In the last chapter, we will focus on these kind of trajectories to understand the occurrence of divergences in the delay time distribution (of an electron entering a magnetic billiard channel).

\subsubsection{Diffusive Transport}

Transport inside a sample is considered to be diffusive if the elastic mean free path of the conducting electrons is much smaller than the dimensions of the sample. In classical diffusive regime the electron coherence length is much smaller than the sample length, so the interference effects due to the wave nature of the electron can be neglected. In the quantum limit (when the phase coherence length of the sample is of the order of the sample length) conductance fluctuations and other effects can be observed. The classical diffusive conductance of a wire is related to the electron concentration $n_{e}$ and the elastic 
scattering time $\tau_{e}$ by

$$
\sigma=\frac{e^{2} n_{e} \tau_{e}}{m^{*}} .
$$

\section{Classical size effects}

In small samples, like wires with a small width, the boundary can have a large effect on the resistivity. In general there will be two types of scattering at the boundary of the sample. The particles can get reflected specularly (i.e., they follow the laws of reflection) or they can get reflected at random (diffusive scattering). In the former case the momentum of the particle is conserved along the wall, while the momentum is reversed along the perpendicular to the wall. Therefore, the conductivity does not get affected due to specular reflection. While in the later case the scattering at the boundary can be considered as an additional scattering center. The nature of reflection (specular or diffusive) depends of the roughness of the boundary and the Fermi wavelength of the electron. In general if the Fermi wavelength is much smaller than the roughness then the scattering is diffusive. Therefore, in metallic conductors the scattering is diffusive due to small Fermi wavelength whereas in semiconductor samples the scattering can be specular due to large value of Fermi wavelength.

\section{Diffusive motion in a magnetic field}

Due to the Lorenz force, in presence of a magnetic field, the electron trajectories follow a circular orbit. In the limit of moderate magnetic field so that

$$
2 r_{c} \simeq W
$$

we find an increased probability that the electrons will hit the boundary Therefore, the electrons which move along the wire get diverted towards the boundary. A further increase in the magnetic field $r_{c} \ll W$ will reduce the probability to hit both the boundary and to reverse its forward movement. As a result the resistance get decreased. As we would see a moderate magnetic field can be enough to bring in backscattering making the dynamics of the electrons diffusive.

\subsection{Analysis and Synopsis}

It was Feynman's idea which bring in revolutions to the field of getting useful work done out of thermal fluctuations. But the study did not remain limited to the field of biological motors and spread to the areas of driven Hamiltonian systems. In Hamiltonian systems we no longer rely on the thermal fluctuations but the underlying chaotic dynamics of the system supports directedness. Due to the diffusive characteristic of an electron in presence of a moderate magnetic field the electron inside a 1D wire (e.g., a chain of quantum dots) can have diffusive motion. Nevertheless we can de-symmetrise 
the boundary of the wire so that it can support a directed transport inside the chaotic sea (even at thermal equilibrium). In the next (second) chapter we study two toy models of quasi-one-dimensional billiard chains (waveguide or channel) in the presence of a perpendicular magnetic field to show this kind of directed motion. The geometry of the waveguides consists of one clean wall scattering the electrons specularly (obeying laws of reflection). The other wall of the channel is distorted in a periodic manner resembling the shape of semicircular discs. The presence of the magnetic field makes some of the electron trajectories to skip regularly in a single direction along the clean wall of the channel. In the other direction there is strong back scattering and the dynamics is chaotic. Consequently all the dynamical quantities like the average transport velocity or the spreading of the distribution of particles is different for the two directions of transport. For these kinds of systems a suitable value of magnetic field will lead to a mixed phase space where all the trajectories are either chaotic or regularly skipping leaving behind little space for periodic trajectories. This results in an asymmetric phase space with a boundary separating the region of chaotic trajectories from the region of regularly skipping ones. As a result of this difference in phase space and the time reversal symmetry breaking (because of the magnetic field) we are able to show that a system in thermal equilibrium can exhibit directed chaos (ratchet like motion). The main feature of this chapter is the calculation of an analytical expression for the asymptotic transport velocity of any chaotic trajectory, the dispersion of the velocities about the mean value and the analysis of results at certain limits of the scatterer. The contents of this chapter and the following chapter are based on the work published in Ref. [40].

The third chapter is dedicated for the study of the aspects of chaotic transmission when one of the walls of the billiard chain is disordered while the other remains clean (no disorders or whatsoever). We will show that for such chaotic systems the net transport resulting from all the chaotic trajectories cannot be zero but it decreases to a finite constant. As one of the walls of the channel is clean, the moving electrons hitting this wall will get reflected specularly. The characteristic of the other wall can be thought of as a wall with high degree of roughness or having closely packed random etching. Thus the electrons getting reflected from this wall violates the laws of reflection and scatter randomly. This random reflection renders the dynamics of the trajectories to behave chaotically. The beauty of this model is that we can use few parameters of these chaotic trajectories to obtain the analytical expressions for the asymptotic chaotic velocity and the diffusion constant. This would have been impossible in case of earlier models (of chapter 1) without numerics.

Due to increase in the study of conductance in mesoscopic nanostructures, in the fourth chapter, we extend the study of the random billiard model (studied in the third chapter) to understand the conductance (in terms of transmission) in a finite wire. Using the fact that some of the trajectories transport in a single direction without any change in the property of trajectories indefinitely (trajectories which get reflected specularly only from the clean wall of the channel), we calculate the asymptotic value of the transmission. For the calculation of the decrease in the transmission to this value we will use several methods. Initially we use the model of a one-dimensional biased random walk to show that for 
very large system length, the transmission decreases exponentially with a characteristic length scale. However the prediction of the pre-factor cannot be determined correctly from this model. To determine the pre-factor we will use the biased correlated random walk where at each point along the line of walk the reflection probabilities are direction dependent. Then using a transfer matrix formalism we will calculate the transmission probabilities and therefore the correct pre-factor, by recognizing that the asymptotic transmission has to be exclusively through regular skipping orbits only. In addition to these we provide another simple method using few recurrence relations and the transmission at a finite length of the system to obtain the transmission at any lengths of the system. Theoretically the method of persistent random walk is quite interesting as it provides various aspects to the length scales of the system. The transmission mechanism in this chapter are mainly based on the work published in Ref. [41].

Owing to the study of time delays in mesoscopic systems, showing universal properties in the distribution of time delays, the fifth chapter is dedicated to the study of the distributions of the time taken by a particle entering at one end of a finite disordered channel before being ejected out at any one of the ends of the channel. Depending on the initial condition, a particle can come out of the system in several ways. They are the following ::

1. If the electron enters the channel through the regular skipping orbits, it will only transmit through the channel (in a single direction).

2. When the electron enters in the direction of regular trajectories but with an initial condition supporting chaotic trajectories, it can transmit or get reflected from the channel. However if the system length is very large their is a high probability that it will get reflected.

3. A particle can transmit in the direction of chaotic flow. There is alway a finite probability for this. However because of the equilibrium condition some has to get reflected as well. Depending on weather the particle get reflected or transmitted we will have different distributions.

We will show that for large system sizes the probability to get reflected at any time is independent of the length of the system. However the probability of transmission at any length of the system has different kinds of distributions for different directions of transport. When the direction of transport is chaotic the distribution shows a peak at the position of chaotic velocity but we observe pulsating behavior in the probability when the direction of transmission is opposite (to the chaotic trajectories). This shows that the directedness of the chaotic trajectories and the regular ones is retained in the distribution of delay times. This is particularly interesting from the view of physics. Because here we have a system which behave differently to the time responses for different direction of transmission even though the total transmission does not depend on the direction of transport. Moreover we qualitatively and quantitatively analyze the nature of distributions using a the biased diffusion equation (in case of chaotic trajectories) and the nature of trajectories (in case of regularly skipping trajectories). 


\title{
Chapter 2
}

\section{Magnetic Billiard Chain with Periodic Structures}

\author{
"Everything that can be counted does not necessarily count; everything that counts cannot \\ necessarily be counted."
}

Albert Einstein (1879-1955)

\subsection{Introduction}

Recently there is a great interest to understand transport phenomena in mesoscopic nanoscale systems. The trend is due to growing demand for smaller and smaller devices. But as the system size decreases, it shows quantum effects, starting from conductance fluctuations and localization effects in a disordered wire to ballistic properties. The ballistic properties are studied using quantum dot structures where the length scale of the system is much smaller than the electron mean free path. These ballistic dots can be viewed as the analog of classical scattering billiards. There electrical properties has attracted a great amount of interest to probe for quantum chaos [42]. With the technology in hand it has been possible to create mesoscopic billiards where the electron motion is essentially ballistic [43]. In some mesoscopic systems the behavior of macroscopic observables can be much more complicated and may be related to the classical dynamics of the system. The transport in such systems can arise due to equilibrium or non-equilibrium phenomena. The non-equilibrium phenomena can arise due to the presence of a driving [28]. It is the properties of this driving which can show a directed transport in such systems. This phenomena of directed transport was originally the idea of biological systems where a uni-directional flow of particles is observed in presence of stochastic noise. Although this idea did not remain with the biological motors it spread to be implemented in condensed matter systems forming a part of so called deterministic ratchets. In non-equilibrium systems this has been modeled 
with an explicit time dependent potential. It has been shown that the driving can break the symmetry in the phase space, which could result in directed transport. Most often such systems contain either regular islands or explicit difference in phase space structures, like variation of momentum distribution [36, 44], providing a net non-zero chaotic transport.

However there is lot to be done in equilibrium systems. Most of the study in equilibrium systems have been done mainly in billiard systems or mesoscopic structures (array of dots/anti-dots). It has been shown that directed transport can be achievable in these systems if the phase space is chaotic and contains two disjoint invariant components in phase space [45]. Nonetheless there are numerical evidences that systems with weaker dynamical stochasticity may exhibit normal transport [46, 47, 48, 49], and systems such as a class of triangular billiards may be mixing [50, 51]. There is also numerical evidence of diffusive transport in a class of dynamical systems with no dynamical chaos (in the sense of exponential separation of nearby trajectories) using polygonal billiards [52]. In mesoscopic systems the study is very wide, due to applications in field of quantum computation [53] to solid-state devices. Recently there is evidence of ratchet effects in molecular wires [54]. In contrast, we use much simpler models of a class of periodic Sinai billiards in presence of a perpendicular magnetic field to show that directed transport of chaotic trajectories can be achievable. The directed transport of chaotic trajectories was a consequence of broken time reversal symmetry (due to presence of magnetic field) and the structural geometry of the system. It is due to these two factors that the equilibrium transport, at certain magnetic field, can be broken into two disjoint sets one set containing essentially chaotic trajectories and the other invariant set containing regularly skipping orbits. The net effect will show in having a directed flow of chaotic trajectories in a single direction. Using the ergodic properties of the system and a simple sum rule we show that the asymptotic transport velocity remains a constant independent of the origin of a chaotic trajectory. Even though most of the work done earlier are based on numerical figures, we attempt for the first time to provide an analytical expression, in terms of the system parameters, for the average chaotic transport velocity.

These types of directed chaos systems has also been studied by others in different contexts. In [55] K. B. Efetov discussed quantum disordered problems with a direction (imaginary vector potential) using weakly asymmetric or non-Hermitian random matrices. These set of problems were named "directed quantum chaos". But in our models we will see that the chaotic trajectories do travel in a single direction. The equivalence can be established by a quantization of the chaotic part of our system.

\subsection{Model of Periodic semi-circular Scatterers}

The initial toy model we studied is shown in figure 2.1. The system consists of a waveguide of infinite length in which one wall is perfectly straight, $y_{1}(x)=b$ and the opposite wall is distorted 
periodically, $y=y_{2}(x) \geqslant 0$ with

$$
y_{\max }=\max _{x} y_{2}(x)<b
$$

In this model this is achieved by a chain of semi-circular obstacles (scatterers) with radius $R=y_{\max }$. The centers of any two consecutive scatterers are separated by a length $a$ (in other words the periodicity of our system is $a$ ). In addition to these geometric parameters we have an external magnetic field $B$ perpendicular to the plane of the system. We know from classical mechanics that for Hamiltonian

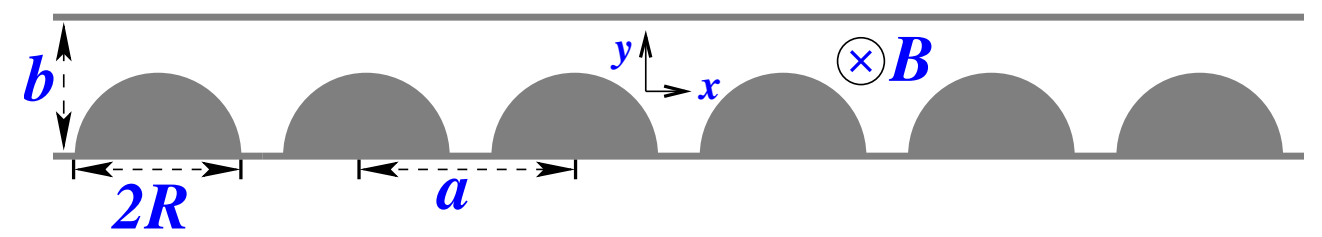

Figure 2.1: The model of the magnetic billiard with periodic semi-circular scatterers of radius $R$. The displacement between the center of two nearby scatterers is $a$ and the width of the waveguide is $b$. A magnetic field perpendicular to the plane of the system distinguishes the movement of electrons from one direction (left to right) to the other (right to left) (for an example see figure 2.2).

systems the total energy of the system is conserved. As there is no potential in the system the total energy is equal to the kinetic energy i.e.,

$$
E=\frac{1}{2} m v_{0}^{2}
$$

where $m=$ mass and $v_{0}=$ magnitude of the velocity of the particle inside the system. Therefore we will consider the dynamics of our model on the energy shell corresponding to the velocity $v_{0}$. For numerical calculations we will use dimensionless units in which $v_{0}=b=1$, i.e., we measure the velocity in units of $v_{0}$, lengths in units of $b$ and time in units of $b / v_{0}$. As a consequence, the geometric length of a trajectory is equal to the elapsed time. Furthermore we consider electrons as our billiard particles. Due to the perpendicular magnetic field the trajectories of electrons inside the waveguide consist of circular arcs with cyclotron radius

$$
r_{c}=\frac{m v_{0}}{e B},
$$

where $e=$ magnitude of the electron charge. This can be derived from the equilibrium of centrifugal force $m v_{0}^{2} / r_{c}$ and the magnetic Lorenz force $e \mathbf{v} \times \mathbf{B}$ at any point on the trajectory. Since the speed of the electron $v_{0}$ has been fixed to unity, we will use this cyclotron radius to parameterize the magnetic field.

The magnetic field leads to a special set of trajectories skipping along the clean wall of the channel (green curve in figure 2.2). Such trajectories keep on moving towards left without any obstructions. We can use simple geometry to describe the transport due to these regular skipping trajectories (See 


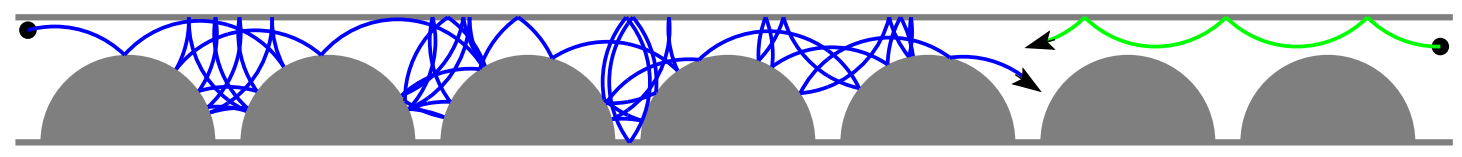

Figure 2.2: In the presence of a magnetic field, perpendicular to the plane of the system, the trajectories of the electrons will consist of a special set of trajectories skipping (green curve) along the clean wall of the boundary whereas the motion along the other direction consists of trajectories hitting the semicircular obstacles (chaotic trajectories). As can be seen these chaotic trajectories (blue curve) contributes to the transport in the opposite direction of the regular skipping orbits.

section 2.4). In contrast, trajectories colliding with the distorted wall of the channel can be chaotic (blue curve in figure 2.2). As can be seen such trajectories will be transporting to the right i.e., opposite to the regular skipping orbits. Therefore, this system shows directed chaos and the transport due to the chaotic trajectories compensates for the regular skipping orbits such that the net transport at equilibrium is zero. We would like to know the average speed contributed by such a chaotic particle using the dynamics of the trajectories in the phase space. This requires the understanding of the dynamics of the electrons in the phase space, which is being discussed in the following section.

\subsection{Phase Space Structure}

Since the geometry of our system is 2-dimensional, the phase space of the system can be described by the position $(x, y)$ and the momentum $\left(\overrightarrow{p_{x}}, \overrightarrow{p_{y}}\right)$ of the electron at any point inside the available space. However we know that the energy is conserved, i.e.,

$$
E=\frac{1}{2 m}\left(p_{x}^{2}+p_{y}^{2}\right)=\text { constant } .
$$

This provides us with one constraint and reduces the number of phase space variables to three. Since the momentum is a vector variable and can be described by its magnitude and the corresponding angle

$$
\vec{p}=\overrightarrow{p_{x}}+\overrightarrow{p_{y}}=p(\hat{i} \cos \varphi+\hat{j} \sin \varphi)
$$

we will use the position $(x, y)$, and the angle $\varphi$ between the velocity vector and the $x$-axis (figure 2.3) to describe the phase space point on the energy shell. In these coordinates, the invariant measure for a small volume component in the phase space is

$$
d \mu=d x d y d \varphi
$$


At equilibrium, since all the positions and momenta are equally probable, the total phase space is uniform and will be filled by the orbits skipping along the clean wall of the channel and the chaotic trajectories (trajectories hitting the scatterers). However the structure of the phase space will differ for different values of the cyclotron radius. To get a general impression of the phase space we can construct a Poincaré section by marking $y$ and $\varphi$ whenever a trajectory crosses the boundary of a unit cell, $x=0$ $\bmod a$, i.e., whenever the horizontal position of the trajectory, $x$ is halfway between two obstacles. Depending on the direction of crossing (left to right for positive $x$-velocity, $v_{x}>0$ and right to left for negative $x$-velocity, $v_{x}<0$ ) we will obtain two panels for the Poincaré section. The details of this construction is described below.

\subsubsection{Construction of Poincaré Map}

A surface of section, also called a Poincaré section, is a way of presenting a trajectory in ndimensional phase space in a (n-1)-dimensional space. By picking one phase element constant an intersection surface is obtained. The dynamics generates a map from the surface of section to itself. For the construction of this Poincaré Map in our system we choose the constant phase space element to be the position between two scatterers $(x=0 \bmod a)$. When a trajectory crosses this point we note the value of $y$ and $\varphi$. Then the value of $y$ is plotted against $\sin \varphi$ to obtain the desired map. The reason for choosing $\sin \varphi$ is the following. Consider a trajectory passing through the desired value of $x$ (between the mid point of any two semi-circular scatterer) at a point $y$ with the velocity vector making an angle $\varphi$ with the $x$-axis (figure 2.3). Then for small changes in the co-ordinate of the particle, $d x, d y$ and $d \varphi$,

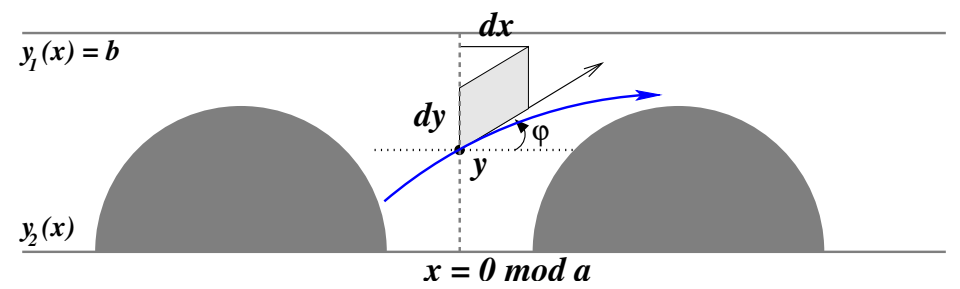

Figure 2.3: Geometry showing the construction of Poincaré map. See details in the text.

the probability $d s$ that an electron will leave at an angle $\varphi$ with the normal to the surface is provided by the Knudsen cosine law,

$$
d s \propto d \mu \cos \varphi .
$$

In other words, the number of particles crossing at an angle $\varphi$ at a particular value of $x$ will be

$$
d \mu_{\perp}=d y d \varphi \cos \varphi
$$


$d \mu_{\perp}$ provides us the invariant measure of the resulting Poincaré Map. In our model, at equilibrium, all the points inside the available phase space volume are equally probable. Therefore, if we fix the position $x$ then we need to plot $y$ against $\sin \varphi$ for the construction of Poincaré map in order to show that there is uniform distribution of points. As already discussed, depending on the sign of velocity $v_{x}=v_{0} \cos \varphi$ of the electron, the Poincare map will consist of two panels. One for the positive and another for the negative values of the velocity $v_{x}$.

We have fixed the width $b$ and the velocity $v_{0}$ of the electron to unity. Therefore, if we fix the radius $R$ of the semicircular scatterer, then we remain with only one parameter namely the external magnetic field (or the cyclotron radius $r_{c}$ ) which can be varied at will. Below we describe qualitatively the appearance of various types of phase space structures for different values of magnetic field.

\section{Case 1 : Special case of a moderate magnetic field $\left(r_{c}>b\right)$}

When the strength of the magnetic field is moderate such that the cyclotron radius $r_{c}>b$, we can observe mainly two kinds of trajectories. One consists of regular trajectories skipping along the clean wall of the waveguide without hitting the semicircular scatterers, while the other consists of trajectories which hit the semicircular scatterers. Such trajectories can be considered as chaotic trajectories. Figure 2.2 shows two such typical trajectories and figure 2.4 shows the Poincaré map for this case of a moderate magnetic field. The scattered points which are seen to be uniformly distributed in both the panels of the plot is a result of a single chaotic trajectory while the continuous looking lines on the negative panel of the Poincaré map is the result of different regular skipping orbits. The Poincaré map shows that
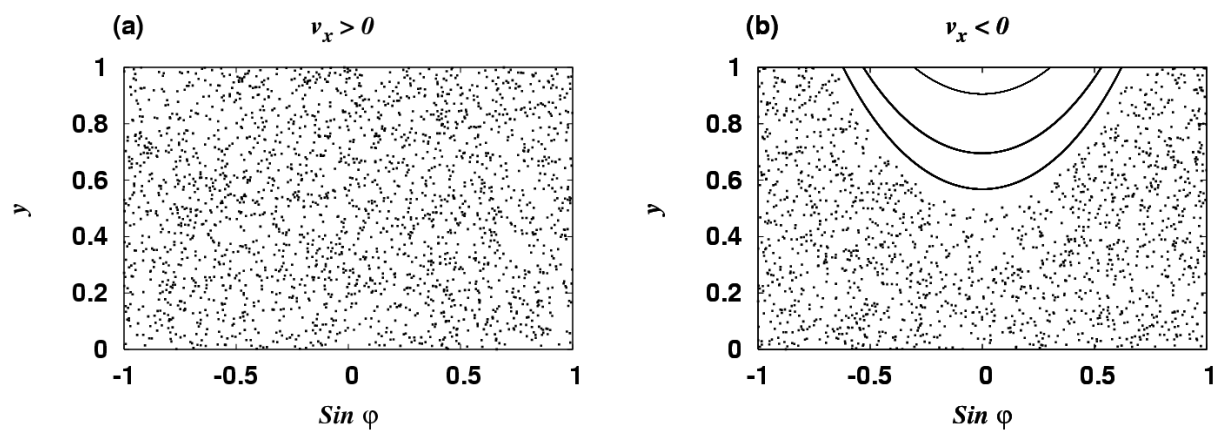

Figure 2.4: Phase space portrait of the system shown in figure 2.1, $y$ and $\varphi$ are shown for $x=0 \bmod a$ and (a) $\cos \varphi>0$, (b) $\cos \varphi<0$. The data represent one chaotic and three skipping trajectories. The uniformity in the density of the chaotic region confirms the ergodicity property of the chaotic phase space. Parameters used for the construction are $a=1.2 b, R=0.5 b$ and $r_{c}=2 b$.

the entire phase space consists of mainly two kinds of trajectories: the skipping trajectories remaining entirely on the negative panel of the Poincare map and the chaotic trajectories which are observed in both directions of the Poincaré map, both separated by a common boundary. The presence of mainly 
two kinds of trajectories leaving behind very negligible space for regular islands inside the chaotic sea makes this case very special. Latter on (see section 2.5) we will use this feature of the phase space in order to calculate an average transport velocity for the chaotic trajectories.

\section{Case 2 : Case of a stronger magnetic field}

If the magnetic field is strong enough then we can observe regular islands in the chaotic sea. Such regular islands inside a chaotic sea are a consequence of periodic orbits inside the system. This is confirmed in figure 2.5. These kind of features are prominent if the value of cyclotron radius is such that $(b-R) / 2<r_{c} \leqslant b$. Trajectories near to such periodic trajectories remain longer and longer near to them and influence the transport dramatically. With the increase in the magnetic field, the number of periodic islands increases in number. As we increase the magnetic field further $r_{c} \leqslant(b-R) / 2$ the scenario changes due to appearance of pinned cyclotron orbits. Also in this case parts of the regular skipping orbits is seen to have a positive component of velocity $v_{x}=v_{0} \cos \varphi$. This corresponds to a very strong magnetic field which we discuss below.

Case 3 : A very strong magnetic field $\left(r_{c} \leqslant(b-R) / 2\right)$

If the magnetic field is such that $2 r_{c}=b-R$ then we can have at least one trajectory pinned to a point inside the system. This trajectory will make $y=b$ and $y=b-R$ as its two tangents. If the magnetic field is increased further then the number of pinned circular orbits grow. Thus the components of the velocity along the direction of the waveguide ( $x$-axis) oscillates about zero. Also we can observe some phase space component of the regular skipping orbits, which on an average transport in the negative direction, can be seen filling some of the regions in the positive panel of the Poincaré map. For example, this will be the case if the center $\left(x_{c}, y_{c}\right)$ of the regular skipping orbits remains below the upper wall of the channel i.e., if the regular skipping orbit is described by a point on the trajectory $(x, y, \varphi)$ then $y_{c}$ is such that $y_{c}=y-r_{c} \cos \varphi<b$. Such trajectories get reflected from the upper wall of the channel with a steep angle $\varphi>3 \pi / 2$. The occurrence of pinned orbits and the positive phase space components for the negatively transporting skipping orbits is confirmed in figure 2.6. The pinned electrons will not play any major role in equilibrium directed transport of electrons inside the waveguide. Nevertheless there will be transport arising from the edge states. These edge states play a prominent role in conduction of electrons inside a wire [56, 57].

\section{Case 4: Very low magnetic field $\left(r_{c} \rightarrow \infty\right)$}

In the other extreme, the strength of the magnetic field can be very low. Therefore the electrons would feel very low influence from the presence of the magnetic field. So, all the electron trajectories will look nearly like straight lines. When the perpendicular magnetic field is exactly equal to zero, 

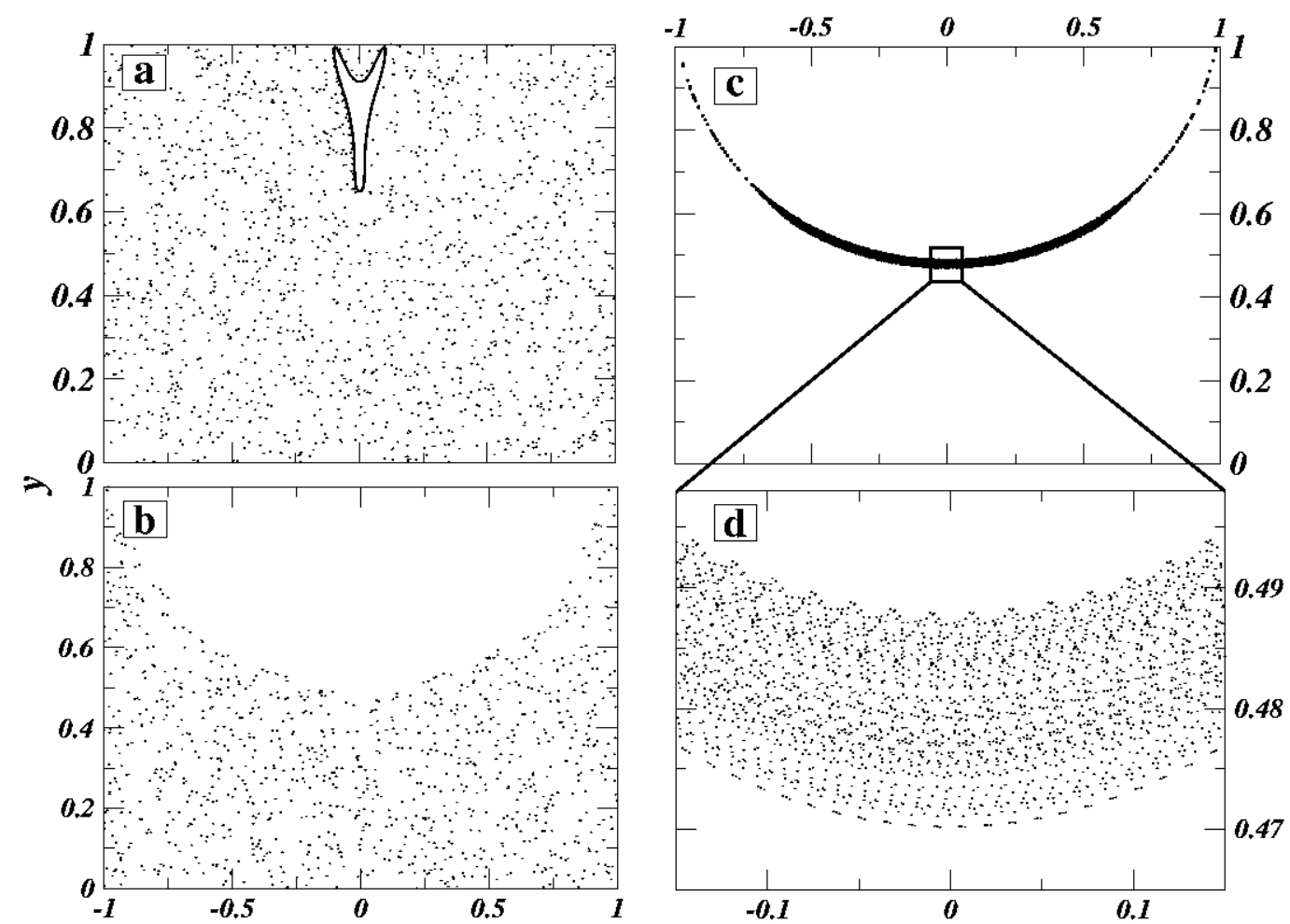

$\operatorname{Sin} \varphi$

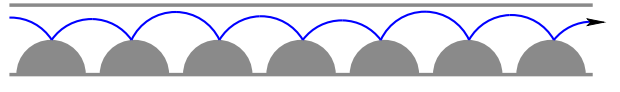

(e)

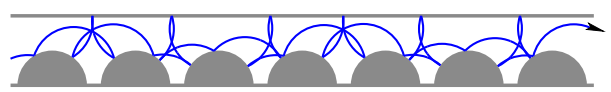

(g)

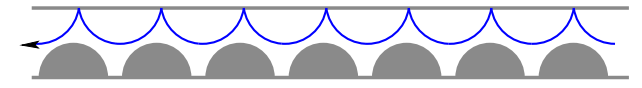

(f)

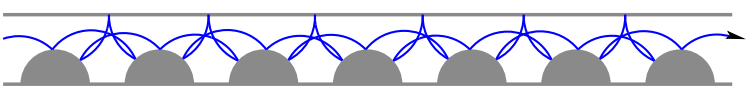

(h)

Figure 2.5: For high magnetic field ( $r_{c}=0.7$ for (a) and (b)) we can observe the occurrence of regular islands inside chaotic sea as in (a). Such type of periodic islands inside chaotic sea can occur due to periodic trajectories as shown in examples (e), $(\mathrm{g})$ and $(\mathrm{h})$. In particular, the black boundary near the border of the regular island in (a) corresponds to the Poincare map of the trajectory shown in (e). (c) and (d) (plotted for $r_{c}=0.6$ ) shows the Poincaré map of a typical chaotic trajectory (f) which keeps on moving in the direction of regular skipping orbits without being hit by the scatterer for a very long time. The occurrence of such islands and long range sticking orbits can significantly change the properties of electronic transport. The parameters used for (a)-(g) are $a=1.2, b=1.0, R=0.5$. The cyclotron radius used for $(\mathrm{g})$ is $r_{c}=0.6$, while (h) have been plotted for $a=1.5, b=1.0, R=0.5, r_{c}=0.7$. The plot in (a) is for $\cos \varphi>0$ while the others (b),(c) and (d) are for $\cos \varphi<0$. 


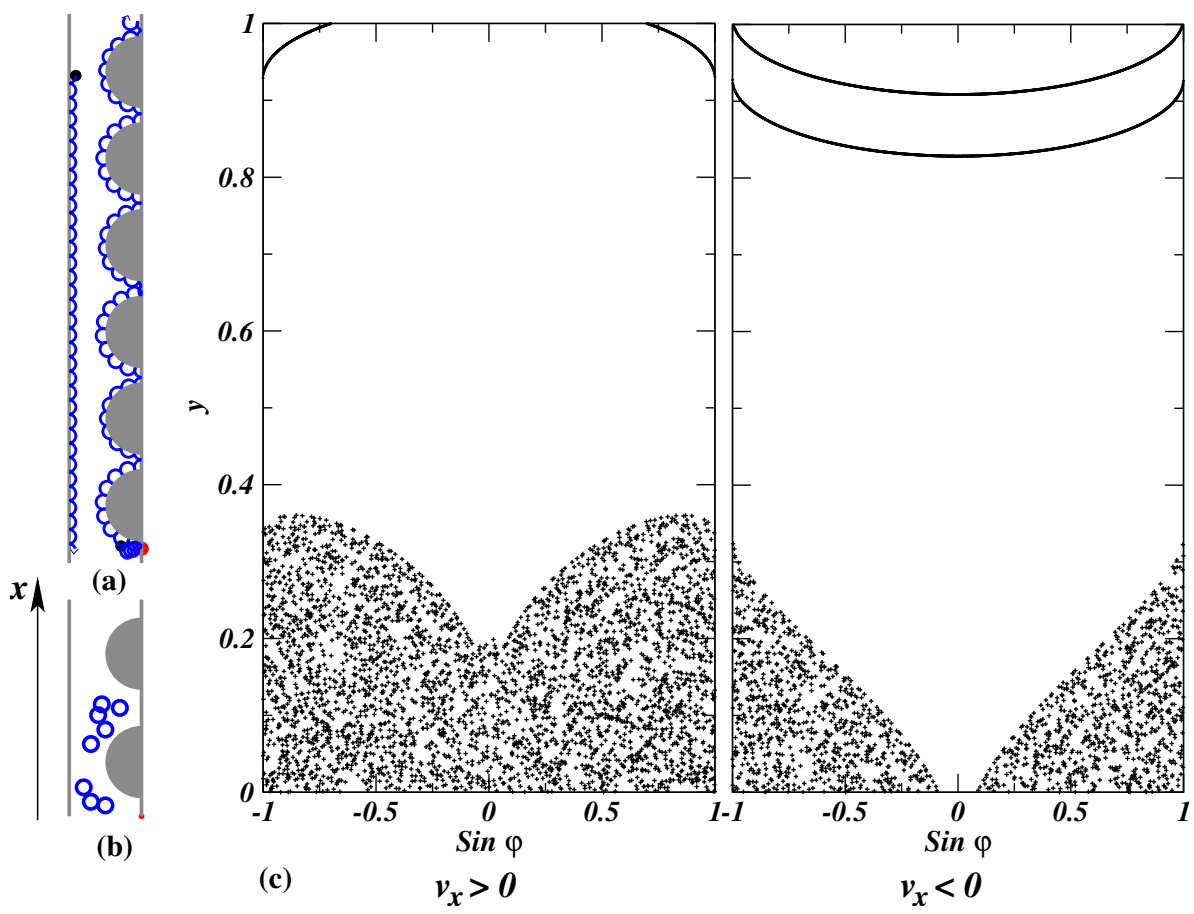

Figure 2.6: (a) Two transporting trajectories and (c) the corresponding Poincaré map for the value of cyclotron radius $r_{c}=0.1$ and the scatterer radius $R=0.5$. The parameters used for the system are $a=1.2$ and $b=1$. The Poincaré map in the negative direction of transport is for two regular skipping orbits. The empty looking regions, in the Poincaré map, are filled by pinned electrons as in (b). One regular skipping trajectory is seen in both panels of the Poincaré map. A regular skipping orbit will be seen in the positive panel of the phase space provided the center $\left(x_{c}, y_{c}\right)$ of the regular skipping orbit is such that $y_{c}<b$.

the trajectories of the electrons will consist of straight lines. For no magnetic field we can argue that the net transport for any chaotic particle is zero along the waveguide. The argument is following. With no magnetic field inside the system, dynamics of the system becomes time-symmetric i.e., for any trajectory moving in any direction at any point in the phase space, we will have a counter moving

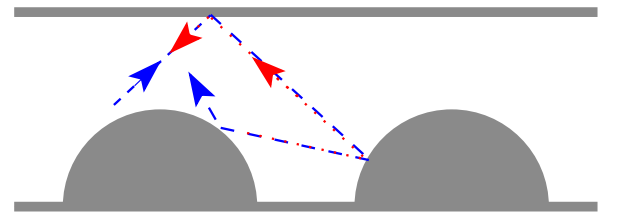

Figure 2.7: When the magnetic field is zero, for each trajectory moving in any direction there is always another trajectory moving in opposite direction, the result is a null average transport. 
trajectory moving in opposite direction at the same point in the phase space (Figure 2.7). This is equivalent to say that for any momentum vector in the phase space, we will have another momentum vector contributing in the opposite direction. The net result is no average transport inside the system. But if the magnetic field is very small we have the net momentum vector of the chaotic trajectories not zero, but very small. This will result in a net transport of chaotic trajectories in a single direction, with a very low average velocity along the direction of the channel. This can be seen from the construction of Poincaré map for a chaotic trajectory. In figure 2.8, we have shown a trajectory and the corresponding Poincaré map for the same value of parameters used for the construction of figure 2.6. The only difference is, this time we have used a very large value for the cyclotron radius $r_{c}=1000$.
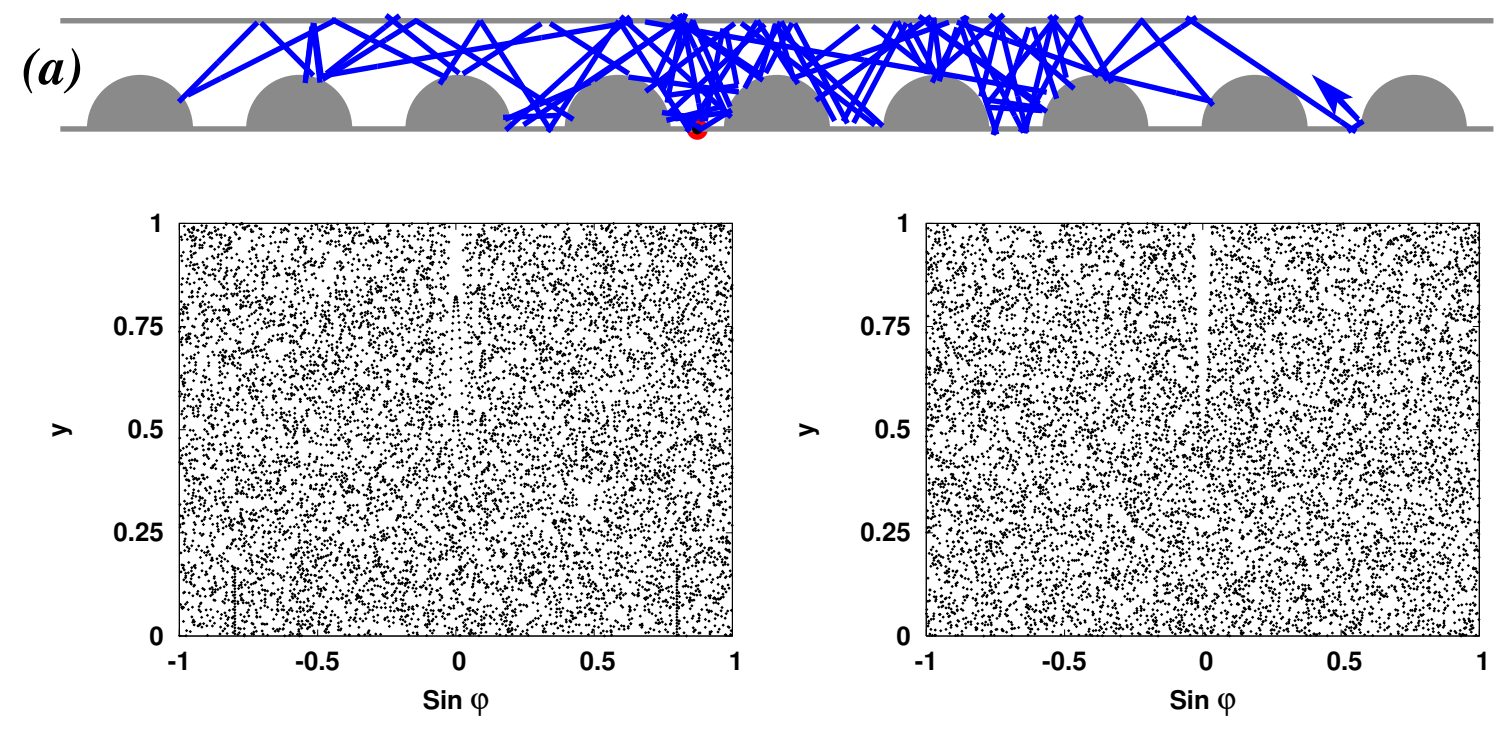

(b)

$$
v_{x}>0
$$$$
v_{x}<0
$$

Figure 2.8: (a) The trajectory of an electron and (b) the corresponding Poincare section when the magnetic field is very small. The value of cyclotron radius, $r_{c}=1000$ and other parameters of the system are same as in figure 2.6. This shows that almost all of the phase space are filled by a single chaotic trajectory leaving very little space for regular skipping orbits. Therefore, the average velocity of the chaotic trajectories will also be very small.

There are significant differences in the Poincaré sections of very large (figure 2.6) and very small (figure 2.8) magnetic field. In the former case, we observe most of the regions in the Poincaré map, are filled by pinned cyclotron orbits. Whereas in the latter case we do observe some unfilled regions as well (when $|\sin \varphi| \sim 0$ ). But they are very small. The cause can be explained as follows. In motion from left to right (i.e., for a chaotic trajectory) if the velocity vector has an angle nearly equal to zero to the $x$-axis, and the initial $y$ value of the trajectory is above $R$, it will travel longer and longer distance before 
it can get scattered by a scatterer. Therefore, the Poincaré section for these trajectories will be restricted to a very large value of velocity near to $\cos \varphi \sim 1$ (or $\sin \varphi \sim 0$ ). In the opposite direction (i.e., when we have motion from right to left or $v_{x}<0$ ), the unfilled regions are filled by regular trajectories which keep on moving in a single direction without much distortions on its path. We also can observe that a chaotic trajectory nearly fills the entire domain of the Poincaré section (both in the positive and the negative panels). Thus, for a chaotic electron, the average velocity arising from the positive direction of travel nearly cancels the motion in the opposite direction. Using this we conclude, for very small value of magnetic field (or large value of cyclotron radius) the net velocity for the chaotic trajectories will always remain close to zero. It can be verified from the plot of average velocity $\left\langle v_{x}\right\rangle=\left\langle v_{0} \cos \varphi\right\rangle$ as a function of time. This is shown in figure 2.9 for different initial conditions, for several trajectories, of an electron inside the phase space. When the magnetic field is moderate we have seen the phase space mainly consisting of two components one chaotic and another regularly skipping. This case of very low magnetic field belongs to this category of mainly two components in the phase space. Therefore, this can be treated more elaborately which has been discussed in section 2.5

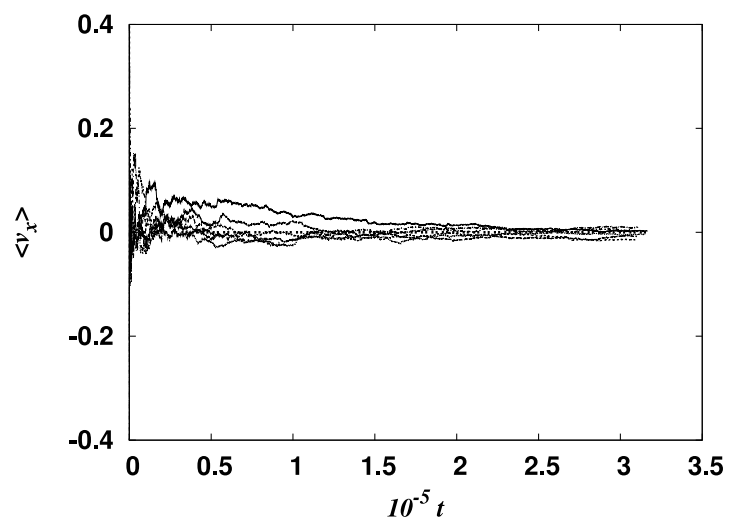

Figure 2.9: Variation of average velocity (for the parameters used in figure 2.8) for electrons starting with different initial conditions as a function of time. It shows that the value of average chaotic transport velocity (for almost all chaotic trajectories) remains close to zero for large cyclotron radius.

We are interested in the quantitative understanding of the chaotic transport in case of a moderate magnetic field when the phase space mainly consist of two components. This will involve the understanding of the regular skipping orbits (as the net equilibrium transport of the chaotic and the regular skipping orbits cancel each other). Therefore, we need to discuss the dynamics of the regular skipping trajectories. The following section deals with these skipping orbits. 


\subsection{Dynamics of regular skipping orbits}

The electrons which do not get scattered from the semi-circular obstacles will keep on moving in a single direction after getting reflected from the clean wall of the channel. Because of this skipping nature, we call them the regular skipping orbits (or simply regular orbits). These trajectories are different from the periodic orbits forming islands inside the chaotic sea. These skipping orbits will have a second constant of motion besides the energy, namely the $y$-component of the current arc's center $y_{c}$ (see figure 2.10)

$$
y_{c}(y, \varphi)=y-r_{c} \cos \varphi .
$$

Therefore, these skipping orbits constitute a regular (integrable) component of the phase space which comprises all phase space points satisfying the condition

$$
\cos \varphi \leqslant \frac{y-R}{r_{c}}-1
$$

that is all arcs whose lowest point is still above the top of the obstacles, $y_{c}(y, \varphi)-r_{c} \geqslant y_{\max }$. It is clear that trajectories with such initial conditions can never be scattered. Nonetheless, one might argue

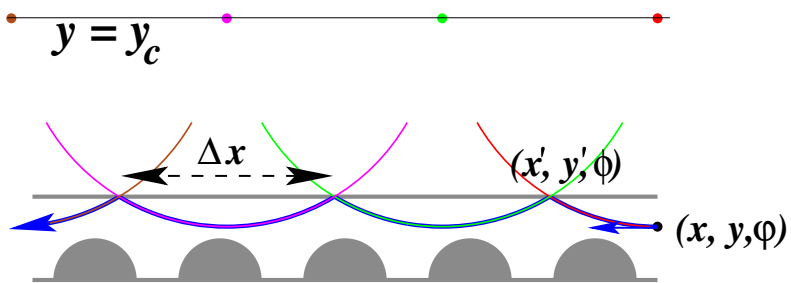

Figure 2.10: The $y$-component of each arc's center of regularly skipping orbits is conserved through the entire length of the trajectory and the $x$-component of such trajectories will have a constant difference (longitudinal extension $\Delta x$ ) between any two nearby equivalent points on the trajectory.

that there can exist skipping orbits violating the condition in equation (2.10). Say the lowest point of the current arc is below $y_{\max }$ but falls into the gaps between two obstacles (see an example in figure 2.5 (f)). In spite of that, if one continues the trajectory it will finally hit one of the scatterers, at least if the longitudinal extension $\Delta x$ of the arcs is not rationally related to the spatial period $a$ of the system. Thus, exceptional skipping orbits within the chaotic component might exist but must have measure zero in phase space.

To calculate the average velocity of a regular skipping orbit, let's assume the point $(x, y, \varphi)$ is in the regular skipping component of the phase space and this trajectory gets reflected at an angle $\phi$ from the upper wall of the channel. Then the points $(x, y, \varphi)$ inside the system and the point $\left(x^{\prime}, y^{\prime}=b, \phi\right)$ (See 
figure 2.10) on the upper wall of the channel are related by

$$
\begin{aligned}
\cos \phi & =\frac{b-y}{r_{c}}+\cos \varphi \\
y^{\prime} & =b \\
x^{\prime} & =x+r(\sin \varphi-\sin \phi)
\end{aligned}
$$

These relations can be derived from the constancy of the center of the trajectory on that section of the orbit. Now using the variable $\phi$ we can calculate the horizontal length traveled by the skipping trajectory between two consecutive reflections

$$
\Delta x(\phi)=2 r_{c} \sin \phi,
$$

during which the electron will travel for a time equal to the length of the arc of that section

$$
\Delta l(\phi)=2 r_{c}(\phi-\pi)
$$

This is shown in the geometrical construction of a section of a trajectory in figure 2.11. Dividing the

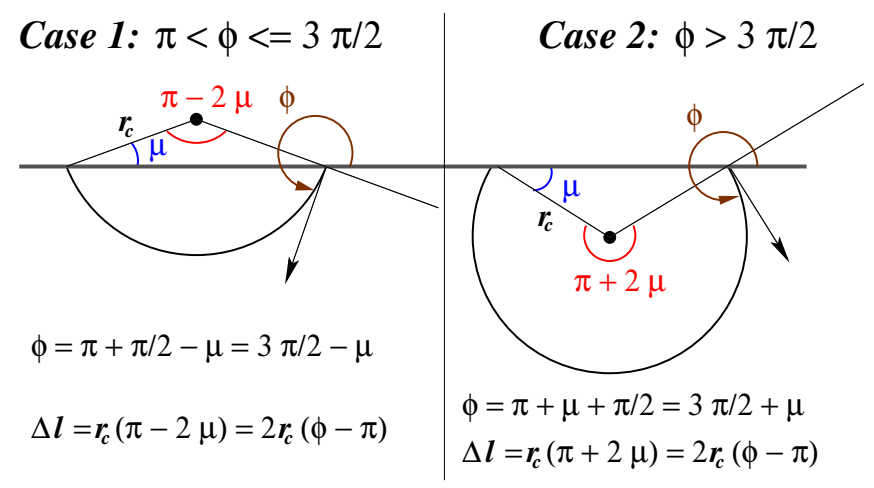

Figure 2.11: Calculation of time of travel for a section of arc in case of regular trajectories with nonzero average velocity. Since the speed $v_{0}$ is unity, the time for a section of the trajectory $=$ total length of the arc for the same section $(\Delta l)$.

total length of the channel into $N$ number of sections each measuring length $\Delta x$ during which the time of travel will be $\Delta l$, will provide the average velocity of the particle

$$
\left\langle v_{\text {reg }}(\phi)\right\rangle=\langle\cos \varphi\rangle_{\text {reg }}=\frac{L}{T}=\frac{N \Delta x(\phi)+\delta x}{N \Delta l(\phi)+\delta l}=\frac{\Delta x(\phi)+\delta x / N}{\Delta l(\phi)+\delta l / N}
$$

where $\delta x$ and $\delta l$ contributes to the shifting of the trajectories in mapping the entire length of the channel $L$ into individual sections of the trajectory and $T$ defines the total time of travel for the electron to pass 
through this length, starting from the initial point $(x, y, \varphi)$. As the time approaches infinity $T \rightarrow \infty$, the distance between the points $(x, y, \varphi)$ and $\left(x^{\prime}, b, \phi\right)$ will be very small compared to the total length of the trajectory $\delta x / N \rightarrow 0, \delta l / N \rightarrow 0$ and thus can be neglected in the calculation of average velocities. Therefore,

$$
\left\langle v_{\mathrm{reg}}(\phi)\right\rangle \approx \frac{\Delta x(\phi)}{\Delta l(\phi)}=\frac{2 r_{c} \sin \phi}{2 r_{c}(\phi-\pi)}=-\frac{\sin \mu}{\mu}, \quad(N \rightarrow \infty),
$$

where $\mu=\phi-\pi$ is the angle made by the trajectory if we measure it from the direction of negative $x$-axis. Thus the average velocity for such a trajectory approaches quickly to a constant which depends only on the initial starting angle at the upper wall of the channel (provided the length of system is very

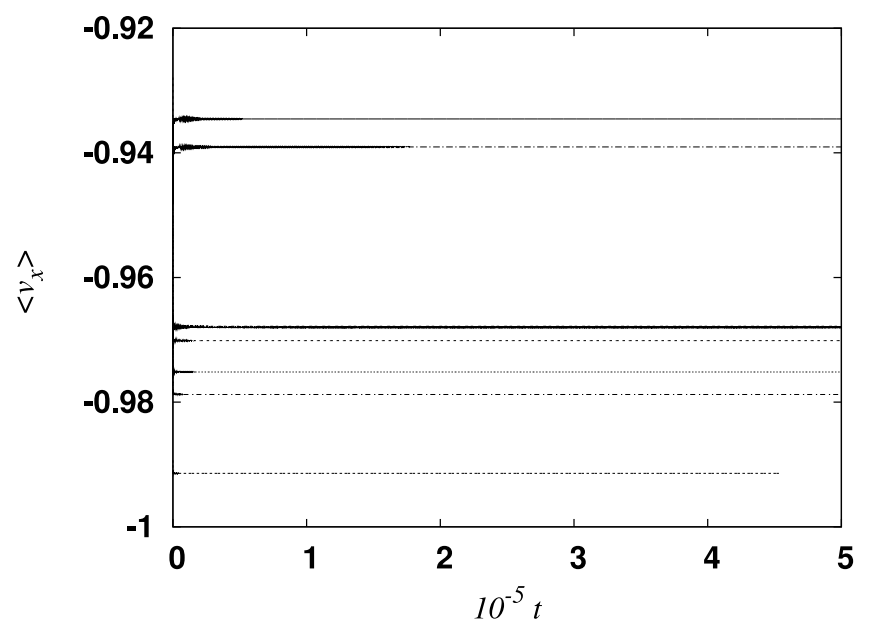

Figure 2.12: Velocity dependence of eight different regular skipping orbits as a function of time for different initial conditions shows a constant value depending on the initial position of the electron on the phase space.

large). This is displayed in figure 2.12 for eight different trajectories. The figure also confirms that the average velocities of the regular skipping trajectories are always negative, independent of the strength of the magnetic field (as long as the trajectory remains regularly skipping). This is a consequence of our convention of the direction of magnetic field (If we change the direction of the magnetic field, it will result in a change of the sign for the velocities of the regular and chaotic components). Moreover the maximum value of $\varphi$ allowed at any point $y$ for a regular skipping trajectory can be obtained from equation (2.10)

$$
\varphi_{\max }=\arccos \left(\frac{y-R-r_{c}}{r_{c}}\right) .
$$

But if $y=b$ (in performing the transformation from $(x, y, \varphi)$ to $\left(x^{\prime}, b, \phi\right)$ or if the trajectory starts at the 
upper wall of the channel) then the maximum allowed angle

$$
\phi_{\max }=\arccos \left(\frac{b-R-r_{c}}{r_{c}}\right) .
$$

Thus, the value of the average velocity, of minimum magnitude, for the regular trajectories

$$
\left\langle v_{r}(\phi)\right\rangle_{\min }=\frac{\sin \phi_{\max }}{\phi_{\max }-\pi}=-\frac{\sqrt{(b-R)\left(2 r_{c}+R-b\right)}}{r_{c} \arccos \left(\frac{R+r_{c}-b}{r_{c}}\right)} .
$$

On the other hand, the minimum value of angle allowed for a trajectory starting at $y=b$ is equal to $\pi$. This will provide the maximum magnitude for the average velocity of the regular skipping trajectories

$$
\left\langle v_{r}(\phi)\right\rangle_{\max }=\lim _{\phi \rightarrow \pi^{+}} \frac{\sin \phi}{\phi-\pi}=-1 .
$$

This is reflected in the distribution of velocities (figure 2.13) for the regular trajectories having a maximum cutoff point around the average velocity, $\langle v\rangle \sim-0.915$ and a minimum cutoff point at $\langle v\rangle=-1$. These results have been obtained by substituting $b=1, R=0.5, r_{c}=2$, in the equations (2.18) and (2.19). To obtain the distribution in figure 2.13, for the average velocities of the regular skipping orbits

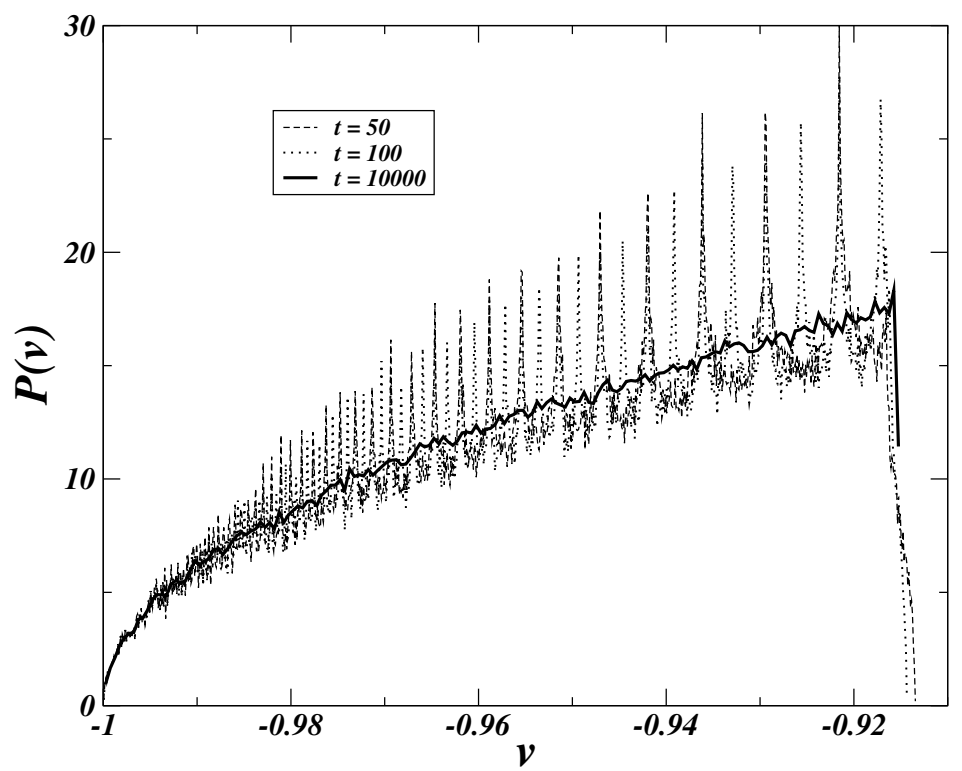

Figure 2.13: Velocity distribution of $N=5 \times 10^{5}$ skipping orbits equally distributed in phase space at different positions of time for the parameter values $b=1, a=1.2 b, r=2 b$ and $R=0.5 b$. For shorter time scales we can observe the appearance of peaks (dotted and dashed lines) in the distribution, while for very large times the distribution follows a smooth curve (dark line). 
along the direction of channel, we simulate $N=5 \times 10^{5}$ number of regularly skipping orbits equally distributed in the phase space of the regular region, for the set of parameters $b=1, a=1.2 b, r_{c}=2 b$ and $R=0.5 b$, and make a distribution plot of the average velocities at various time scales. As the trajectories transport inside the channel we note the time and the corresponding average velocity at that time. The data are then gathered to make the required statistical distribution. For moderate magnetic field $\left(r_{c}>b\right)$, the distribution of regular velocities confirms

1. The existence of an upper limit and a lower limit (as provided by the equations (2.18) and (2.19) respectively) for the magnitude of velocities of regular skipping orbits,

2. No matter whatever the time may be the distribution of average velocities, for all regular trajectories remains almost a constant (apart from the appearance of peaks for shorter times) with variation of time.

As can be seen from the distribution, for finite time the distribution shows occurrence of peaks in the distribution. The question arises, if there is some pattern of these peaks or they come out at random? The initial impression from the distribution is that they have some pattern. As the time increases by a factor of two, the number of peaks seems also getting increased by two. In general we can say that, between the peaks occurring at time $t$, new peaks emerge in between these peaks at time $2 t$. The reason for peaks at shorter times is a consequence of exact divisibility of the total length of the channel by the horizontal distance $\Delta x$ i.e., when $L=N \Delta x, N$ being an integer. But, we will wait until the fourth chapter to understand the occurrence of these peaks, where we will study the delay time distribution of chaotic and regular transmission for a finite length of the waveguide.

Equation (2.18) has one more significance. For a real number value of the square root, the argument of the square root function must be positive definite i.e., $(b-R)\left(2 r_{c}+R-b\right) \geqslant 0$. For transport to occur we need the width of the channel greater than the scatterer radius, $b>R \Rightarrow b-R>0$. Therefore for a real value of velocity we need

$$
\left(2 r_{c}+R-b\right) \geqslant 0 .
$$

This says that for equation (2.18) to be valid we need $2 r_{c} \geqslant b-R$. This will ensure us a minimum value for the magnitude of velocity with expression given in equation (2.18). When this inequality fails i.e., $2 r_{c}<b-R$, in other words we allow the existence of pinned orbits inside the system the minimum value of average velocity becomes zero (due to the existence of pinned orbits which have average transport zero inside the channel).

\subsection{Dynamics of Chaotic Orbits}

Unlike the regular skipping orbits, which can be treated simply on the basis of the angle made by the trajectory at the upper boundary $\phi$, the treatment of chaotic trajectories is not easy. The reason 
being the multiple reflections from the scatterers, which render them chaotic. However if the phase space of the system is simple (as in the case of a moderate magnetic field), the statistical properties of the chaotic orbits can be handled more easily. Thus, for simplicity we assume no periodic islands inside the chaotic sea and a moderate magnetic field inside the system. In other words the magnetic field is such that $r_{c}>b$ and the phase space mainly consists of two manifolds.

At moderate magnetic field $\left(r_{c}>b\right)$, we do not observe any pinned orbits nor do we observe any periodic islands inside the chaotic sea. Therefore, the trajectories inside the system will mainly be either chaotic or regularly skipping. Nonetheless, one might argue that there can exist skipping orbits inside the chaotic sea. Say the lowest point of the current arc is below $y_{\max }$ but falls into the gap between two obstacles or the trajectory is such that it does not get obstructed by the scatterer. In spite of that, if one continues the trajectory it will finally hit one of the scatterers, at least if the longitudinal extension of the arcs is not rationally related to the spatial period $a$ of the system. Thus, exceptional skipping orbits within the chaotic component might exist but must have measure zero in phase space. Hence they can be neglected for the purpose of statistical analysis. Also, as the parameters of the system do not get altered in the course of time, the regular phase space volume and the chaotic phase space volumes remains conserved. Moreover the chaotic phase space volume is ergodic (see figure 2.4). The ergodicity of phase space means we can fill the entire chaotic phase space with a single chaotic trajectory. By starting a chaotic trajectory with almost any initial condition and mapping all the points on the trajectory to the unit cell, as the trajectory evolves with time; if we find that the initial trajectory fills the entire phase space volume of the chaotic area, this will indicate that the chaotic phase space is ergodic. Numerical evidence have been gathered for our model by checking that individual chaotic trajectories cover the available chaotic phase space region uniformly (see figure 2.4). Therefore, as the time grows to infinity, the statistical properties of chaotic trajectories will remain independent of the precise initial condition. To be specific the chaotic trajectories will all acquire an average velocity independent of the initial condition. As the regular trajectories have a negative average velocity, the chaotic trajectories will acquire a positive average velocity such that the net current inside the system remains zero (at equilibrium).

\subsubsection{Calculation of average chaotic transport velocity}

The calculation of average quantities for the chaotic components requires to trace a trajectory for an infinite time. However we can use the underlying equilibrium of the phase space components and the simple phase phase structure of two main components in order to calculate an average velocity for the chaotic trajectories. The condition of equilibrium says that at thermal equilibrium the net current arising from all the components inside the phase space must vanish. Therefore, if we assume $v_{\text {reg }}$ as the average velocity of all the regular skipping trajectories and $v_{\mathrm{ch}}$ is the average velocity of all the chaotic trajectories then by taking into account the periodic islands (per) and pinned (pin) orbits, if any, we can 
write

$$
\sum_{i} \Omega_{i} v_{i}=0
$$

where $\Omega_{i}$ is the phase space volume occupied by the $i$-th component ( $i \in\{\mathrm{ch}$, reg, per, pin $\}$ ) and the sum is over all the individual components of the phase space. Also, the total phase space volume of the unit cell $\Omega_{\mathrm{uc}}$ is the sum of the volumes of the individual components

$$
\Omega_{\mathrm{uc}}=\sum_{i} \Omega_{i}
$$

As the pinned orbits has average transport velocity zero inside the system, the equation (2.21) can be rewritten for the average chaotic velocity

$$
v_{\mathrm{ch}}=\frac{-\left(\Omega_{\mathrm{reg}} v_{\mathrm{reg}}+\Omega_{\mathrm{per}} v_{\mathrm{per}}\right)}{\Omega_{\mathrm{uc}}-\left(\Omega_{\mathrm{reg}}+\Omega_{\mathrm{per}}+\Omega_{\mathrm{pin}}\right)} .
$$

For the case of a moderate magnetic field, as the periodic islands have a measure zero in the phase space, $\Omega_{\text {per }} \rightarrow 0$ and there are no pinned orbits $\Omega_{\text {pin }}=0$ inside the system, the equation (2.23) provides an expression for the average chaotic transport velocity

$$
v_{\mathrm{ch}}=\frac{-\Omega_{\mathrm{reg}} v_{\mathrm{reg}}}{\Omega_{\mathrm{uc}}-\Omega_{\mathrm{reg}}} .
$$

On the other hand, the chaotic phase space is ergodic, which says that as times goes to infinity the chaotic component of the phase space can be entirely filled by a single trajectory. Therefore, the time average chaotic transport velocity for almost all chaotic trajectories converges to the corresponding phase space average

$$
\left\langle v_{x}\right\rangle_{\mathrm{ch}}=\lim _{T \rightarrow \infty} \frac{1}{T} \int_{0}^{T} v_{0} \cos \varphi(t) d t=\Omega_{\mathrm{ch}}^{-1} \int_{\mathrm{ch}} v_{0} \cos \varphi d \mu,
$$

where $d \mu=d x d y d \varphi$ is the invariant phase space measure and the integration is over the entire chaotic phase space region. This is illustrated in figure 2.14, In general there is no reason to expect $\left\langle v_{x}\right\rangle_{\mathrm{ch}}=0$ in equation (2.25). However, an average over $\cos \varphi$ is obviously zero, if for any point $(x, y, \varphi)$ contributing in the domain of integration also the point $(x, y, \pi+\varphi)$ with opposite velocity direction contributes. For example this will be the case if the integration domain extends over the entire phase space of the unit cell.

$$
v_{\mathrm{uc}}=\left\langle v_{0} \cos \varphi\right\rangle_{\mathrm{uc}}=\Omega_{\mathrm{uc}}^{-1} v_{0} \int_{\mathrm{uc}} \cos \varphi d \mu=0 .
$$

Hence in a complete chaotic billiard we will have $\left\langle v_{x}\right\rangle_{\mathrm{ch}}=0$, i.e., there will be no directed chaos. Similarly, in any system with a single chaotic component and time-reversal symmetry there can be no 
directed chaos even if stable islands are present. On the other hand directed chaos can exist in fully chaotic systems with time-reversal symmetry if the phase space contains two invariant components [45]. It also has been observed that a fully chaotic Hamiltonian can support directed chaos if the system involves broken spatiotemporal symmetries with an asymmetric diffusion in momentum (nonuniform phase space distribution) [36, 44]. In our system this is not the case, however at moderate magnetic field we can decompose the full phase space into two components namely the chaotic and the regular skipping orbits. Thus

$$
\Omega_{\mathrm{uc}} v_{\mathrm{uc}}=v_{\mathrm{reg}} \Omega_{\mathrm{reg}}+v_{\mathrm{ch}} \Omega_{\mathrm{ch}}=0,
$$

This is the condition of equilibrium which says that the net current inside the system must be zero. Hence it is same as the equation (2.24) and is analogue to the sum rule derived in [28] for the chaotic transport velocity in driven 1D systems. However in the present case of magnetic billiards we can calculate the expression for $v_{\text {ch }}$ explicitly. For this we note that the total phase space volume is the sum of the volumes of the individual components

$$
\Omega_{\mathrm{uc}}=\Omega_{\mathrm{reg}}+\Omega_{\mathrm{ch}},
$$

and in terms of integrations over the phase space domain

$$
v_{\mathrm{reg}} \Omega_{\mathrm{reg}}=\int_{\mathrm{reg}} v_{0} \cos \varphi d \mu, \quad \text { and } \quad v_{\mathrm{ch}} \Omega_{\mathrm{ch}}=\int_{\mathrm{ch}} v_{0} \cos \varphi d \mu
$$

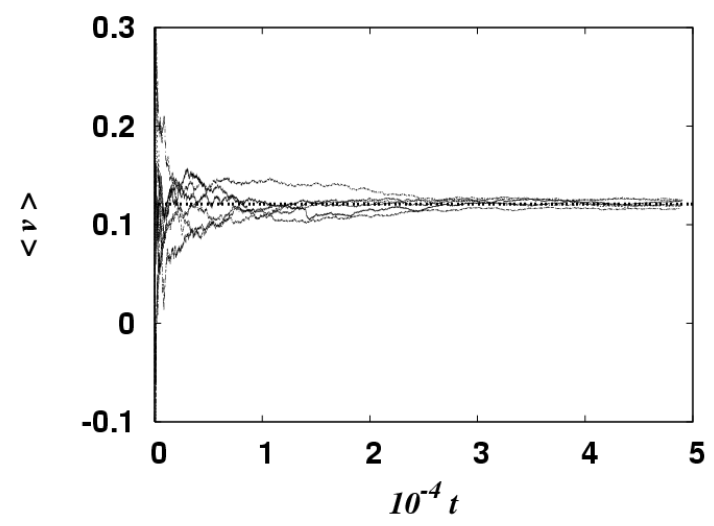

Figure 2.14: For the model of figure 2.1 and parameters of figure 2.4, the time averaged velocities of six different chaotic trajectories are shown as a function of time. For all the trajectories the average velocity approaches the analytical value predicted by equation (2.40) which is indicated by a horizontal line. 
where the integration is over the corresponding domains. Therefore, using equation (2.27), we can write

$$
\int_{\mathrm{ch}} \cos \varphi d \mu=-\int_{\text {reg }} \cos \varphi d \mu \text {. }
$$

Combining equations (2.25), (2.28) and (2.30), we obtain

$$
\left\langle v_{x}\right\rangle_{\mathrm{ch}}=v_{\mathrm{ch}}=-\frac{\int_{\mathrm{reg}} v_{0} \cos \varphi d \mu}{\int_{\mathrm{uc}} d \mu-\int_{\mathrm{reg}} d \mu} .
$$

The total phase space volume of the unit cell

$$
\Omega_{\mathrm{uc}}=\int_{\mathrm{uc}} d \mu=\int_{\mathrm{uc}} d x d y d \varphi=2 \pi\left(a b-\frac{\pi}{2} R^{2}\right)
$$

and the regular skipping trajectories can be distinguished from the chaotic trajectories because they always remain above the semi-circular scatterer placed at the lower boundary. To know which trajectories always remain above $y_{\max }$, we assume a trajectory starting with initial position $(x, y, \varphi)$ in the phase space. Then the $y$-component of the center of this trajectory $y_{c}$ needs to satisfy

$$
y_{c}-r=y-r_{c}(\cos \varphi+1) \geqslant R .
$$

Assuming $\varphi=\pi+\alpha$, we can rewrite the above equation (2.33) as

$$
y-r_{c}(1-\cos \alpha) \geqslant R \quad \text { or } \quad \cos \alpha \geqslant 1-\frac{y-R}{r_{c}} .
$$

With the inequality replaced by equality, this will provide the limiting value for the angle $\alpha$ (and therefore of $\varphi$ ) for which a skipping trajectory, starting at $y$ with initial angle $\varphi$, will have its minimum at the maximum value of the lower wall. This optimum value is

$$
\alpha(y)=\arccos \left(1-\frac{y-R}{r_{c}}\right),
$$

and all the other possible angles defining a regular skipping trajectory starting at $(x, y, \varphi)$ will lie within the closed interval

$$
\varphi \in[\pi-\alpha(y), \pi+\alpha(y)]
$$

The former value can be obtained by noticing that if $\pi+\alpha$ is a regularly skipping point at $(x, y \neq b)$ then there exists another point $\left(x^{\prime}, y\right)$ on the same section of the trajectory with angle $\pi-\alpha$, they being differed by a horizontal distance of magnitude $2 r \sin \alpha$. Also for a trajectory to be regularly skipping the value of $y$ should remain within the maximum of the lower boundary and the minimum of the upper 
boundary i.e., $y \in[R, b]$. So, we can write

$$
\int_{\text {reg }} d \mu(\ldots)=\int_{0}^{a} d x \int_{R}^{b} d y \int_{\pi-\alpha(y)}^{\pi+\alpha(y)} d \varphi(\ldots)
$$

where (...) can be any observable quantity. For calculation of the phase space volume (...) is equal to unity and for transport velocity along the length of the channel $(\ldots)=v_{0} \cos \varphi$. Thus the volume of the regular phase space

$$
\begin{aligned}
\Omega_{\mathrm{reg}} & =\int_{\mathrm{reg}} d \mu=\int_{0}^{a} d x \int_{R}^{b} d y \int_{\pi-\alpha(y)}^{\pi+\alpha(y)} d \varphi=a \int_{R}^{b} 2 \alpha(y) d y \\
& =2 a \int_{R}^{b} d y \arccos \left(1-\frac{y-R}{r_{c}}\right)=-2 a r_{c} \int_{1}^{1-(b-R) / r_{c}} \arccos t d t \\
& =2 a r_{c}(\sin \theta-\theta \cos \theta)
\end{aligned}
$$

where $\theta=\alpha(b)=\arccos \left[1-(b-R) / r_{c}\right]$ denotes the maximum deviation of the limiting skipping orbit from the horizontal. Similarly,

$$
\begin{aligned}
\Omega_{\mathrm{reg}} v_{\mathrm{reg}} & =v_{0} \int_{\mathrm{reg}} \cos \varphi d \mu=v_{0} \int_{0}^{a} d x \int_{R}^{b} d y \int_{\pi-\alpha(y)}^{\pi+\alpha(y)} \cos \varphi d \varphi=-v_{0} a \int_{R}^{b} 2 \sin [\alpha(y)] d y \\
& =-2 a v_{0} \int_{R}^{b} \sin \left[\arccos \left(1-\frac{y-R}{r_{c}}\right)\right] d y=-2 a v_{0} \int_{R}^{b} \sqrt{1-\left(1-\frac{y-R}{r_{c}}\right)^{2}} d y \\
& =2 a r_{c} v_{0} \int_{1}^{1-(b-R) / r_{c}} \sqrt{1-t^{2}} d t=v_{0} a r_{c}[\cos \theta \sin \theta-\arcsin (1)+\arcsin (\cos \theta)] \\
& =v_{0} a r_{c}\left[\cos \theta \sin \theta-\frac{\pi}{2}+\frac{\pi}{2}-\theta\right] \\
& =-a r_{c} v_{0}(\theta-\sin \theta \cos \theta) .
\end{aligned}
$$

Finally substitution of these equations into relation (2.31) yields

$$
v_{\mathrm{ch}}=\frac{v_{0}}{2} \frac{\theta-\sin \theta \cos \theta}{\left[\pi /\left(a r_{c}\right)\right]\left(a b-\frac{\pi}{2} R^{2}\right)-(\sin \theta-\theta \cos \theta)}
$$

for the chaotic transport velocity. This result is confirmed numerically in figures 2.14 .

For small magnetic field (or large cyclotron radius $r_{c} \rightarrow \infty$ ), when our assumption of a two component phase space is justified best, we can approximate equation (2.40) to the leading order in cyclotron 
radius. We find

$$
\theta \approx \sqrt{\frac{2(b-R)}{r_{c}}}
$$

and

$$
v_{\mathrm{ch}} \approx v_{0} \frac{4 a}{3 \Omega_{\mathrm{uc}}} \sqrt{\frac{2(b-R)^{3}}{r_{c}}} .
$$

This shows explicitly that asymptotically with increasing the cyclotron radius, the chaotic transport decreases like a power law with a coefficient $1 / 2$, and vanishes in the absence of a magnetic field $\left(r_{c} \rightarrow \infty\right)$. This confirms the qualitative argument discussed in section 2.3.1, as to why for low magnetic field the average chaotic transport velocity is small inside the channel.

In figure 2.15, we plot the average chaotic velocity from equation (2.40) as a function of cyclotron radius $r_{c}$ and the obstacle radius $R$. For this plot we have fixed the width of the channel to be unity $(b=1)$ and the periodicity of the system $a=1.2 b$. As expected for very low magnetic field (high
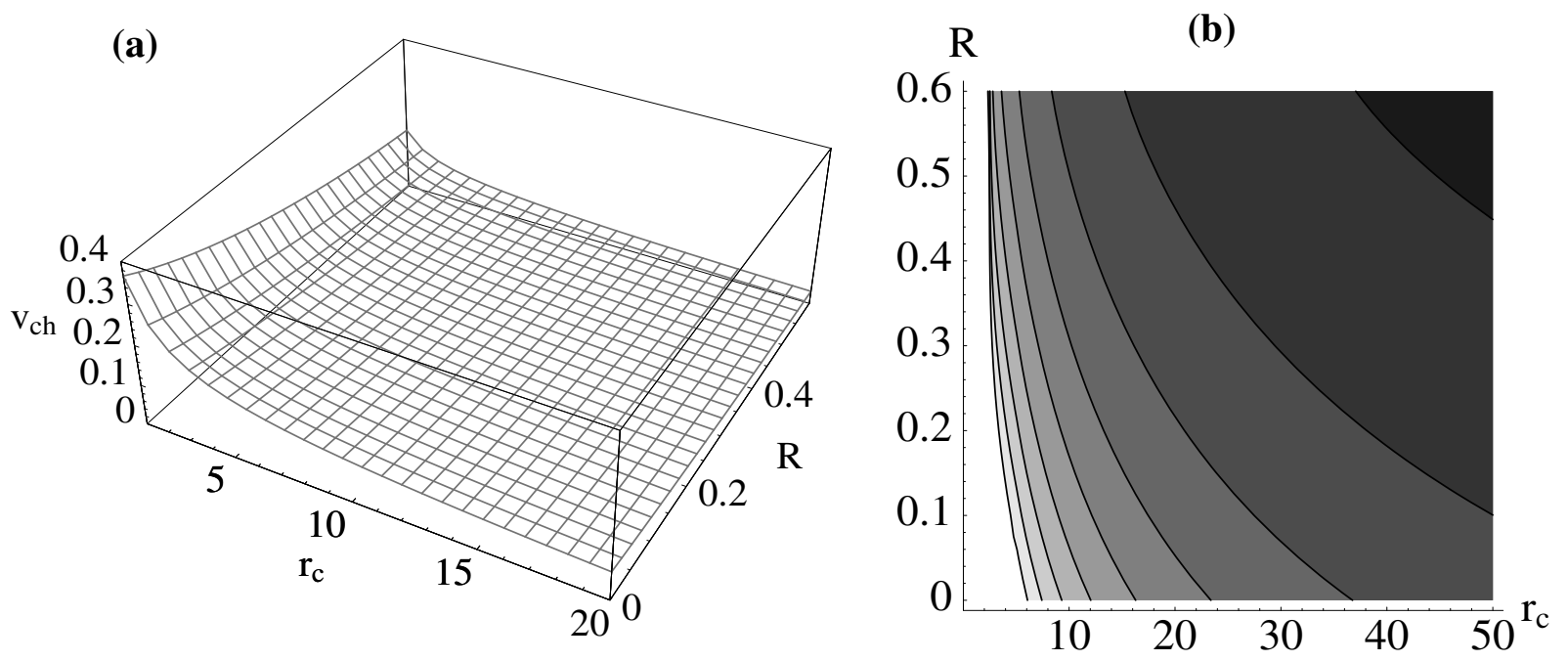

Figure 2.15: (a) Variation of average chaotic velocity (equation (2.40) ) as a function of its parameters namely cyclotron radius $r_{c}$ and the scatterer radius $R$, when $v_{0}=1, b=1$ and $a=1.2 b$. (b) The contour plot of the same function showing equi-velocity lines (larger values are shown with lighter shades) .

cyclotron radius) the average chaotic transport velocity approaches zero as $r_{c}^{-1 / 2}$. As the value of magnetic field increases the system goes towards a more symmetric way of transporting the chaotic electrons and when the magnetic field vanishes the system becomes exact time-reversal symmetric resulting in no net average transport of chaotic electrons. We also can obtain some outlook as to how we can obtain maximum current for chaotic transport. Looking at the figure, we observe that for smaller scatterer radius and smaller cyclotron radius our system supports maximum current. Even when the separation between two neighbor scatterers becomes zero $(R=0.6$ in the figure), we won't obtain 
maximum current. But rather when we allow some space between the scatterers we obtain maximum current inside the system. And for nearly zero scatterer radius i.e., when we have maximum gap between two neighbor scatterers, we obtain maximum average chaotic current inside the billiard chain. The reason being, for very small obstacles the phase space volumes of the chaotic and the regularly skipping trajectories are nearly equal

$$
\Omega_{\text {reg }} \lesssim \Omega_{\text {ch }} \Rightarrow v_{\text {ch }} \lesssim-v_{\text {reg }}
$$

using equation (2.27). This will provide the maximum value for the average chaotic velocity to be

$$
v_{\mathrm{ch}} \approx\left|v_{\mathrm{reg}}\right|
$$

\subsubsection{Dispersion of chaotic trajectories}

Using the equation (2.40) we can precisely calculate the average chaotic transport velocity of many chaotic trajectories at a given moment of time, or the long time average of a typical chaotic trajectory. However this does not explain how a trajectory converges to its asymptotic value. In other words we have no precise information about the dispersion of an ensemble of chaotic trajectories. It is known that for sufficiently fast decay of correlations, the chaotic dispersion is diffusive,

$$
\left\langle\left[x(t)-v_{\mathrm{ch}} t\right]^{2}\right\rangle=D t
$$

$D$ being the diffusion constant. In this case the distribution of time averaged velocities

$$
\bar{v}_{t}=\frac{x(t)-x(0)}{t}
$$

is for long times a Gaussian with variance $D / t$,

$$
P\left(\bar{v}_{t}\right)=\sqrt{\frac{t}{2 \pi D}} \exp \left[-\frac{t\left(\bar{v}_{t}-v_{\mathrm{ch}}\right)^{2}}{2 D}\right]
$$

In figure 2.16 we compare this hypothesis to the chaotic dispersion for the parameters of figure 2.4 Clear deviations from the diffusive behavior are observed in the tails of the distribution and for its higher moments. After iterating $N=5 \times 10^{5}$ trajectories equally distributed in phase space to time $t=10^{3}$ the average chaotic velocity very well agrees with the value predicted by the analytical equation (2.40). However, we can observe asymmetries around $v_{\mathrm{ch}}$ and compared to a Gaussian, large deviations from the mean value are enhanced. For the considered time interval the variance of the distribution $(+)$ is fitted well by the diffusive prediction $D / t$. However with increasing $n$ the higher moments $\left\langle\left(v-v_{\mathrm{ch}}\right)^{n}\right\rangle$ of the velocity distribution deviate more and more from the corresponding time dependent Gaussian 

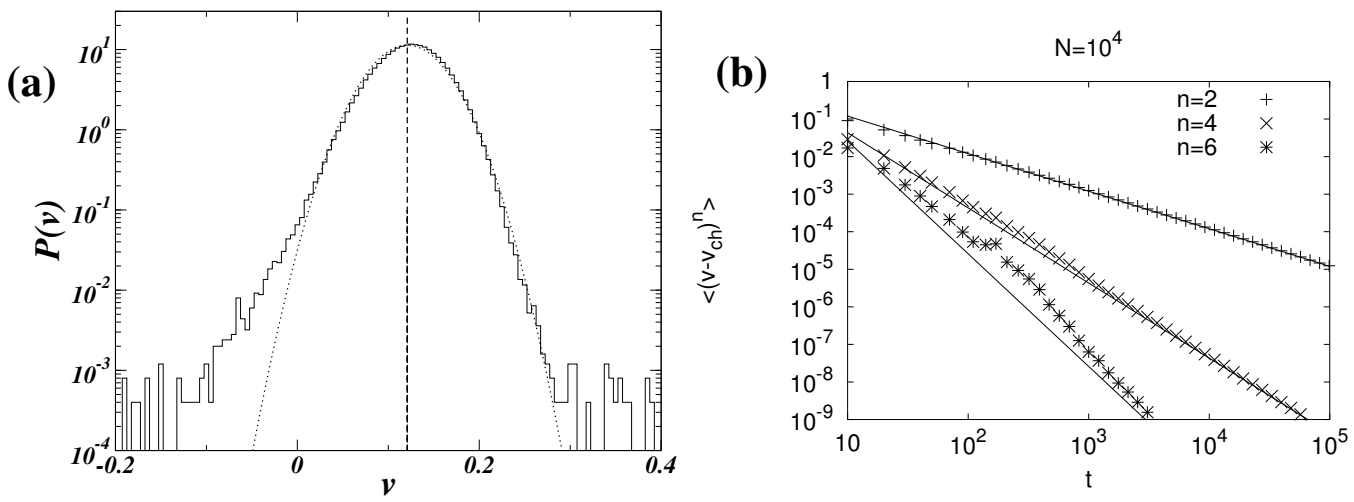

Figure 2.16: (a) The dispersion of average chaotic velocities, for the model of figure 2.1, $N=5 \times 10^{5}$ trajectories have been iterated for time $t=10^{3}$. The resulting distribution of average velocities is displayed as a histogram and compared to a Gaussian with same variance and mean (dotted line). The prediction of equation (2.40) for the average velocity is indicated by a vertical line. The numerical mean value of the distribution differs from that by $6.6 \times 10^{-5}$. (b) shows some of the higher moments of the distribution as a function of time. The straight lines indicate the corresponding moments $\left\langle\left(\bar{v}-v_{\mathrm{ch}}\right)^{2}\right\rangle$ of a diffusively spreading Gaussian with $D=1.2$. Parameters are same as in the construction of figure 2.4

$(X, *$ versus full line). This is no surprise as in systems with a mixed phase space anomalous diffusion is typical. Indeed, in previous studies of directed chaos in such systems the observed velocity distributions were always non-Gaussian [27, 58, 59]. They displayed traces of Lévy walks and anomalous diffusion which were even stronger than those in figure 2.16, and it was not possible to adjust the model parameters such that the directed chaos coexists with normal diffusion. As shown in figure 2.17, few orbits keep on moving in a single direction without being hit by the scatterer. Such periodic trajectories

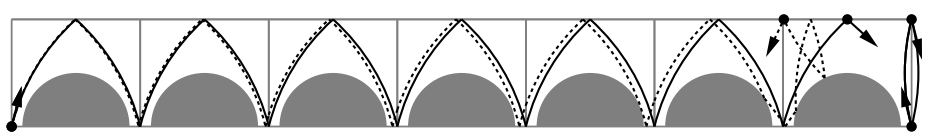

Figure 2.17: Characteristic large deviations arise from marginally stable periodic orbits. The full lines show two marginally periodic orbits. The dotted line is a non-periodic chaotic trajectory which remains in the vicinity of a marginally stable orbit for a long time.

translate along the channel without changing their shape. Nevertheless they will constitute a set of measure zero in the phase space since the angle of the trajectory cannot be varied without destroying the orbit (dotted line in figure 2.17). These trajectories are a source of non-exponential decay of correlations. They lead to anomalous diffusion even in completely chaotic systems like the Sinai or the stadium billiard [60, 61, 62]. In our system generic chaotic trajectories can remain in their vicinity 
for a long time and during these episodes keep the velocity substantially above or below the average. Therefore it is natural that the specifications of geometry has a large impact on the nature of chaotic transport inside the system. Hence it is quite remarkable that in our model a slightly modified geometry leads to a velocity distribution which numerically could not be distinguished from a Gaussian. In figure 2.18 we have repeated the plot of the distributions of velocities for $N=5 \times 10^{5}$ number of chaotic trajectories equally distributed in phase space simulated for time $t=10^{3}$ inside the channel. The results agree with a characteristic Gaussian about the mean value. For this figure we have (i) the semicircular

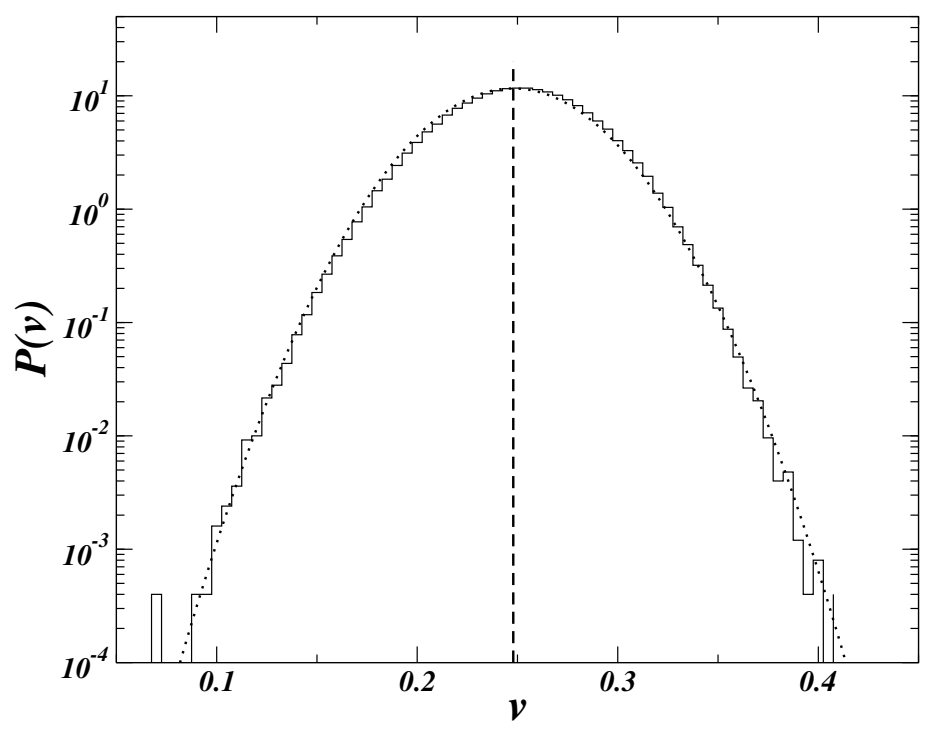

Figure 2.18: Shown here the distribution of velocities for small obstacle radius of $R=0.01 b$ and no separation between any two consecutive semi-circular regions for the model of figure 2.1. The distribution show a Gaussian behavior. The dotted line show the corresponding Gaussian with same mean and variance. The analytical result of velocity from equation (2.40) is shown with a vertical dashed line.

regions have much smaller radius than in figure 2.1 and (ii) neighboring scatterers are tangent rather than separated by a gap (see figure 2.19), so the periodicity of the chain equal to twice the radius of the semi-circular region. Because of this reduction of the gap between any two nearby semi-circular regions the number of un-scattered trajectories gets reduced to nearly zero leaving behind all those trajectories which always get scattered from the semicircular regions. Therefore long range connections of the trajectories also get reduced in number. As a result very few (almost no) trajectories contribute to Lev́y flight like motion in the system. Hence, it is natural that the chaotic dispersion shows much less anomalous diffusion (see figure 2.18) when the specific geometry prevents the existence of marginally stable trajectories.

In contrast, it is not immediately clear why reducing the radius of the scatterers should further 

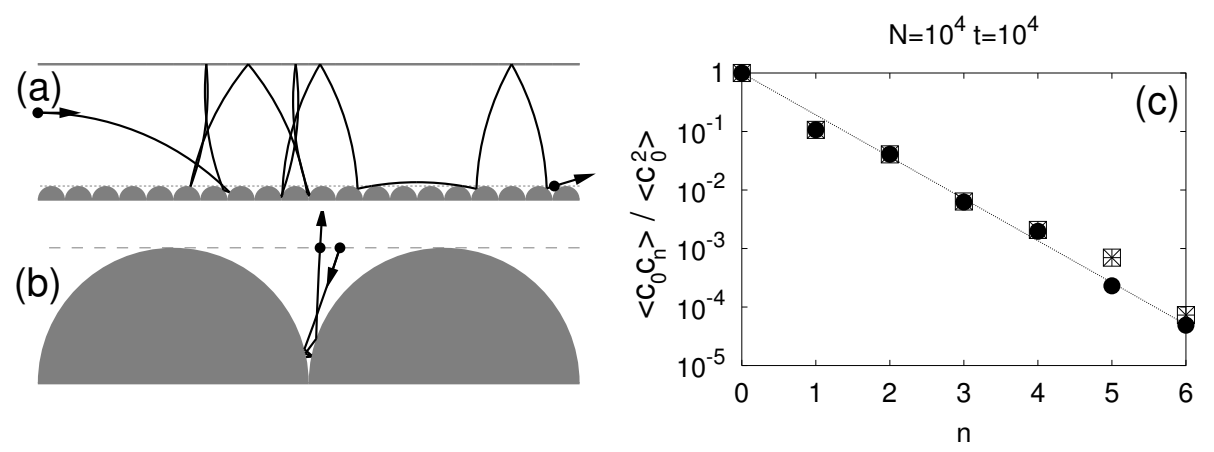

Figure 2.19: (a) A piece of chaotic trajectory is shown for $R=0.1 b$ and $a=2 R$. (b) One particular scattering of this trajectory from the lower boundary is magnified. Note that on this scale the curvature of the trajectory due to the magnetic field is hardly visible. (c) The normalized correlation between consecutive intersection angles of the segments with the line $y=R$ is shown with stars for the model of (a), with empty squares for $R=0.01 b$ and with black dots for the same $R$ but randomized $\xi_{n}$ (see text). The straight line shows the fitted exponential $\exp (-1.65 n)$

suppress anomalous diffusion in our model. In order to find an intuitive explanation for this numerical fact we consider now a different Poincaré section defined by the intersections of a trajectory with the line $y=R$. Note that regular skipping orbits do not at all intersect this line. In analogy to equation (2.8) the invariant measure restricted to the surface of section is now

$$
d \mu_{\|}=d y d \varphi \sin \varphi=d y d c
$$

with $c=\cos \varphi$. Hence, a typical chaotic trajectory is represented in this Poincaré section by points $\left(\xi_{n}, c_{n}\right)$ which uniformly cover the area $[0,1] \times[-1,1] . \xi_{n}=\left(x_{n} / a\right) \bmod 1$ denotes here the position of the intersection relative to the unit cell. Following a transition across $y=R$ from below, $\left(\xi_{n-1}, c_{n-1}\right)$, for a chaotic trajectory, there will always be an intersection from above which is denoted by $\left(\xi_{n}^{\prime}, c_{n}^{\prime}\right)$. Geometry requires $c_{n}^{\prime}=c_{n-1}$.

For small obstacle radius transport is mainly due to the long segments of the trajectory above $y=R$ (figure 2.19). Each segment is either a single circular arc or a combination of two arcs which are identical up to a reflection. Segment $n$ is entirely characterized by the value of $c_{n}$. Therefore the statistics of the $c_{n}$ determine the transport properties. In particular, the velocity dispersion will be diffusive if correlations between consecutive angles decay sufficiently fast. Figure 2.19 (c) shows that for sufficiently small obstacle radius $\left\langle c_{0} c_{n}\right\rangle$ indeed decays very fast as a function of $n$ (exponentially fast to a good approximation). This observation can be understood as follows: As $R \rightarrow 0$, a segment $\left(\xi_{n-1}, c_{n-1}\right) \rightarrow\left(\xi_{n}^{\prime}, c_{n}^{\prime}\right)$ traverses more and more unit cells between its end points. As a consequence, one expects an extremely rapid variation of $\xi_{n}^{\prime}$ as a function of $c_{n-1}$. This suggests to replace $\xi_{n}^{\prime}$ by a 
random variable. Figure 2.19 (c) confirms that this approximation is very good for the parameters of Figure 2.19(a) or even smaller obstacle radius $R$. Note that the argument leading to this randomization does not depend on the value of the cyclotron radius $r_{c}$. In particular, it remains valid also in the absence of a magnetic field, $r_{c}=\infty$.

To summarize this model, for a moderate magnetic field the system sustains either a chaotic trajectory or a regular skipping orbit. Although there will be some periodic trajectories inside the chaotic region they will have measure zero. This simple phase structure helps the chaotic trajectories to be directed into a single direction (opposite of the regular skipping orbits). From now on we will assume that we are working on this regime of magnetic field and try to understand other phenomena that can arise in the system. Before going further let us try to think what will be the criteria for transport inside such systems. If this is the only geometry that could provide a way of chaotic transport in the presence of a magnetic field?

\subsection{Model with Convex Semi-circular Boundary}

In the previous sections we considered a model of periodic semicircular scatterers inside a waveguide to understand the phenomena of directed chaos. To increase the understanding further in other kind of systems we consider a different geometry shown in figure 2.20. In the previous model the boundary is deformed in a semi-circular way such that the net phase space volume available for the electron is decreased. However in the current example we consider the same kind of deformations in the system but with a slight modification such that the net phase space volume $\Omega_{\mathrm{uc}}$ of the system is increased. For simplicity we concentrate on a moderate magnetic field so that the phase space comprises of mainly chaotic and regular regions without much embedded features (like periodic islands) inside the chaotic sea.

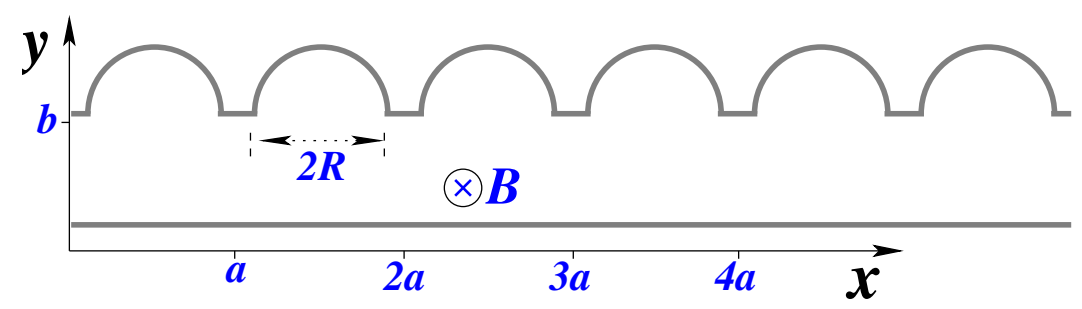

Figure 2.20: Periodic semi-circular regions have been added to the upper boundary of a rectangular waveguide in construction of this model. This increases the total phase space volume available for an electron inside the channel. Parameters used for this model are $b=1, a=1.2 b, R=0.5 b$ and $r_{c}=2 b$. 

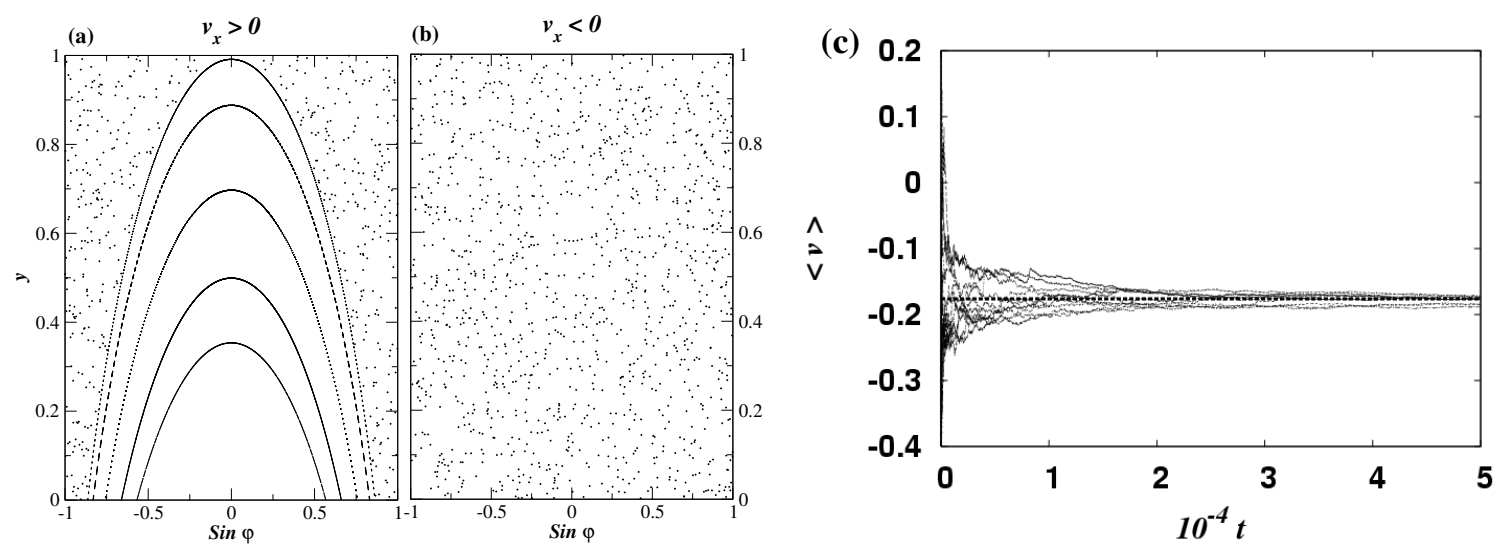

Figure 2.21: Phase space portrait for the parameters of figure 2.20, $y$ and $\varphi$ are shown for $x=0$ $\bmod a$ and (a) $\cos \varphi>0$, (b) $\cos \varphi<0$. The data represent five skipping and one chaotic trajectory. The uniformity in the density of the chaotic region confirms the ergodic property of the chaotic phase space. (c) The time averaged velocities of ten different chaotic trajectories is shown as a function of time. For all the trajectories the average velocity approaches the analytical value predicted by equation (2.56) which is indicated by a horizontal line.

\subsubsection{Average chaotic velocity}

The chaotic phase space of this model is ergodic. This can be confirmed numerically by the construction of a Poincaré map (shown in figure 2.21). Moreover by the value of chosen magnetic field, we can observe that the trajectories, of an electron inside the system are either getting scattered from the deformed upper wall or supports skipping orbits near the lower wall of the channel. However there is a small difference in comparison to the previous model. In the previous model the average chaotic velocity of any chaotic trajectory is positive (asymptotically). This was the result of the counter moving skipping orbits which have a net negative velocity. In contrast this model has a net positive velocity supported by the regular skipping orbits. Therefore, we expect the average chaotic velocity at infinite time should reach a negative value.

Ergodicity in the chaotic phase space suggests that for almost all chaotic trajectories the chaotic transport velocity converges to the corresponding phase space average. This is shown in figure 2.21(c). Therefore, we can use the equation (2.24) or (2.31) to find an expression for the average chaotic transport velocity. These equations involve the calculations of contributions from regular skipping orbits. We note that the maxima of a regular skipping trajectory should always lie below $y=b$ (the minima of the upper wall) i.e, it needs to satisfy

$$
y_{c}+r_{c} \leqslant b \Rightarrow y-r_{c} \cos \varphi+r_{c} \leqslant b .
$$

If the maxima goes beyond this region, then the trajectory will hit the deformed part of the wall; 
the result is a chaotic trajectory. However in this region, it can support some exceptional periodic orbits. They might result from periodic orbits having the longitudinal length relating rationally to the periodicity $a$ of the waveguide (without hitting the deformed wall). Therefore some exceptional periodic trajectories will be present in the chaotic sea but will have measure zero i.e., a small deviation from the initial point will be sufficient to divert the trajectory to become chaotic.

Condition (2.49) ensures that a trajectory starting at $(x, y, \varphi)$ is regular. If we choose the upper limit on the angular component at $(x, y)$ to be $\alpha(y)$, such that the trajectory still remains regular then it must make the minimum of the upper boundary a tangent. This limiting trajectory satisfy

$$
y-r_{c} \cos \alpha(y)+r_{c}=b .
$$

Therefore,

$$
\alpha(y)=\arccos \left(1+\frac{y-b}{r_{c}}\right) .
$$

By symmetry we can come to a conclusion that all those regular skipping trajectories at $(x, y)$ will have angular components within $[-\alpha(y), \alpha(y)]$. Therefore for calculations over regular components we can write

$$
\int_{\text {reg }} d \mu(\ldots)=\int_{0}^{a} d x \int_{0}^{b} d y \int_{-\alpha(y)}^{\alpha(y)} d \varphi(\ldots)
$$

where $(. .$.$) can be any measurable quantity of the phase space. For this second model the total phase$ space volume

$$
\Omega_{\mathrm{uc}}=\int_{\mathrm{uc}} d \mu=\int_{\mathrm{uc}} d x d y d \varphi=2 \pi\left(a b+\frac{\pi}{2} R^{2}\right) .
$$

Using the transformation provided in equation (2.52), we can calculate the volume of the regular skipping components

$$
\begin{aligned}
\Omega_{\mathrm{reg}} & =\int_{\mathrm{reg}} d \mu=\int_{0}^{a} d x \int_{0}^{b} d y \int_{-\alpha(y)}^{\alpha(y)} d \varphi=a \int_{0}^{b} 2 \alpha(y) d y \\
& =2 a \int_{0}^{b} d y \arccos \left(1+\frac{y-b}{r_{c}}\right) \\
& =2 a r_{c} \int_{1-b / r_{c}}^{1} \arccos t d t \\
& =2 a r_{r}(\sin \theta-\theta \cos \theta)
\end{aligned}
$$

where $\theta=\alpha(0)=\arccos \left[1-b / r_{c}\right]$ denotes the maximum deviation of the limiting skipping orbits from the horizontal i.e, it is the maximum angle made by any skipping trajectory which will make the line 
$y=b$ as its tangent. Similarly

$$
\begin{aligned}
\Omega_{\mathrm{reg}} v_{\mathrm{reg}} & =\int_{\text {reg }} v_{0} \cos d \mu=\int_{0}^{a} d x \int_{0}^{b} d y \int_{-\alpha(y)}^{\alpha(y)} v_{0} \cos \varphi d \varphi=a v_{0} \int_{0}^{b} 2 \sin [\alpha(y)] d y \\
& =2 a v_{0} \int_{0}^{b} \sin \left[\arccos \left(1+\frac{y-b}{r_{c}}\right)\right] d y=2 a v_{0} \int_{0}^{b} \sqrt{1-\left(1+\frac{y-b}{r_{c}}\right)^{2}} d y \\
& =2 a v_{0} r_{c} \int_{1-b / r_{c}}^{1} \sqrt{1-t^{2}} d t=a v_{0} r_{c}[\arcsin (1)-\cos \theta \sin \theta-\arcsin (\cos \theta)] \\
& =a v_{0} r_{c}(\theta-\sin \theta \cos \theta)
\end{aligned}
$$

Combining all of these values into equation (2.31), we find

$$
v_{\mathrm{ch}}=\frac{v_{0}}{2} \frac{-(\theta-\sin \theta \cos \theta)}{\left[\pi /\left(a r_{c}\right)\right]\left(a b+\frac{\pi}{2} R^{2}\right)-(\sin \theta-\theta \cos \theta)}
$$

for the average chaotic transport velocity. The result is confirmed in figure 2.21(c). We note that this expression is the same as the equation (2.40) upto the changed expression for the unit cell phase space volume and the value of $\theta$.

To know the behavior of our system at small magnetic field (large cyclotron radius), we make a large $r_{c}\left(r_{c} \rightarrow \infty\right)$ expansion of equation (2.56). We find

$$
\theta \approx \sqrt{\frac{2 b}{r_{c}}}
$$

and

$$
v_{\mathrm{ch}} \approx-v_{0} \frac{4 a}{3 \Omega_{\mathrm{uc}}} \sqrt{\frac{2 b^{3}}{r_{c}}} .
$$

This shows that for no magnetic field there will not be any transport for the chaotic trajectories. This can be concluded on the basis of time-reversal symmetry as well. When there is no magnetic field inside our system, for an electron moving in any direction at a point in the phase space there will be an equal probability moving in the negative direction as well. The net result will be no average velocity for the chaotic trajectories.

\subsection{A comparison of the two models}

We have already noticed the similarities in the expressions of the chaotic transport velocities (equations (2.40) and (2.56) ) due to the change in the unit cell phase space volume and the maximum devia- 
tion angle $\theta$. The change in the phase space volume is due to the cut-out or attachment of a semicircular region into the unit cell and the change in the expression of $\theta$ is due to the placement of the semi-circular regions. Also we have discussed the dispersions of velocities in the previous model. In the earlier model the distribution of velocities, figure 2.16, shows that the large deviations get enhanced from the predicted average chaotic velocity. After iterating same number of trajectories (i.e., $N=5 \times 10^{5}$ number

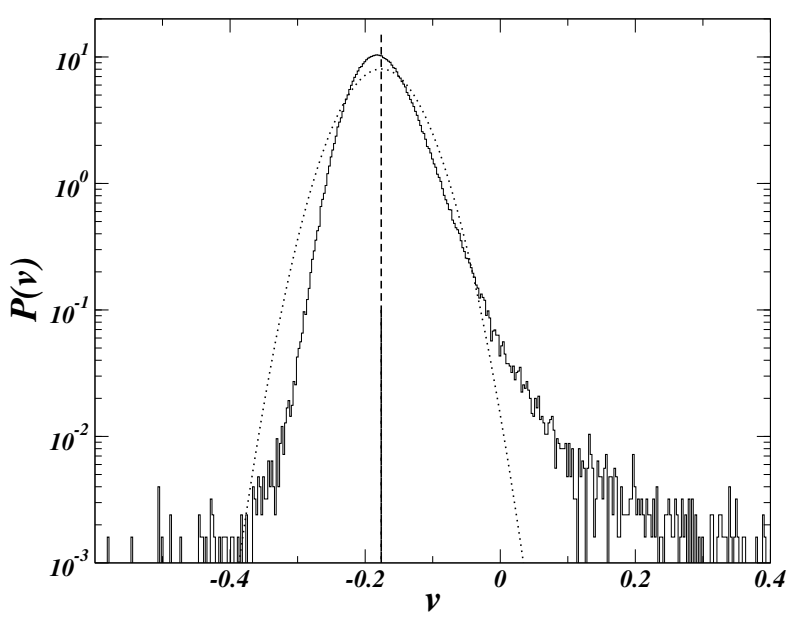

Figure 2.22: The dispersion of average chaotic velocities, for the model of (2.20). $N=5 \times 10^{5}$ trajectories have been iterated for time $t=10^{3}$. The resulting distribution of average velocities is displayed as a histogram and compared to a Gaussian with same variance and mean (dotted line). The prediction of equation (2.56) for the average velocity is indicated by a vertical line. The numerical mean value of the distribution differs from that by $1.9 \times 10^{-5}$. Parameters are same as in the construction of figure 2.4 .

of points equally distributed in chaotic phase space) for the same time $t=10^{3}$, we come to a similar conclusion (see figure 2.22) in case of the second model. Nonetheless there is a small difference in the inclination of velocities towards a particular direction. In the former model, there is a tendency of trajectories to back scatter providing the tail of the distribution enhanced in the direction of negative velocity. In the current model, this is observed in having a forward scattering of the trajectories i.e., large deviations are observed towards the positive direction of velocity. There is one more difference as well. In the previous model we find that the distribution looks more or less a Gaussian about the mean value, but in the latter model we see deviations from this Gaussian behavior even near to the average value. This results from the long-range correlations of chaotic trajectories. In the former model the chaotic correlations decay much faster. This is because there a trajectory is unlikely to avoid a collision with the obstacles (provided $R$ is not very small). However in the present model a chaotic trajectory can make several reflections from the straight part of the boundary segments (segments between the semi-circular regions) before getting scattered.

For a detailed variation of the average velocity with the cyclotron radius $r_{c}$ and the radius of the 
semi-circular region $R$, we plot equation $(2.56)$ as a function of these parameters. This is shown in figure 2.23 for the periodicity $a=1.2 \mathrm{~b}$. We can observe similar behavior as in the earlier model (figure
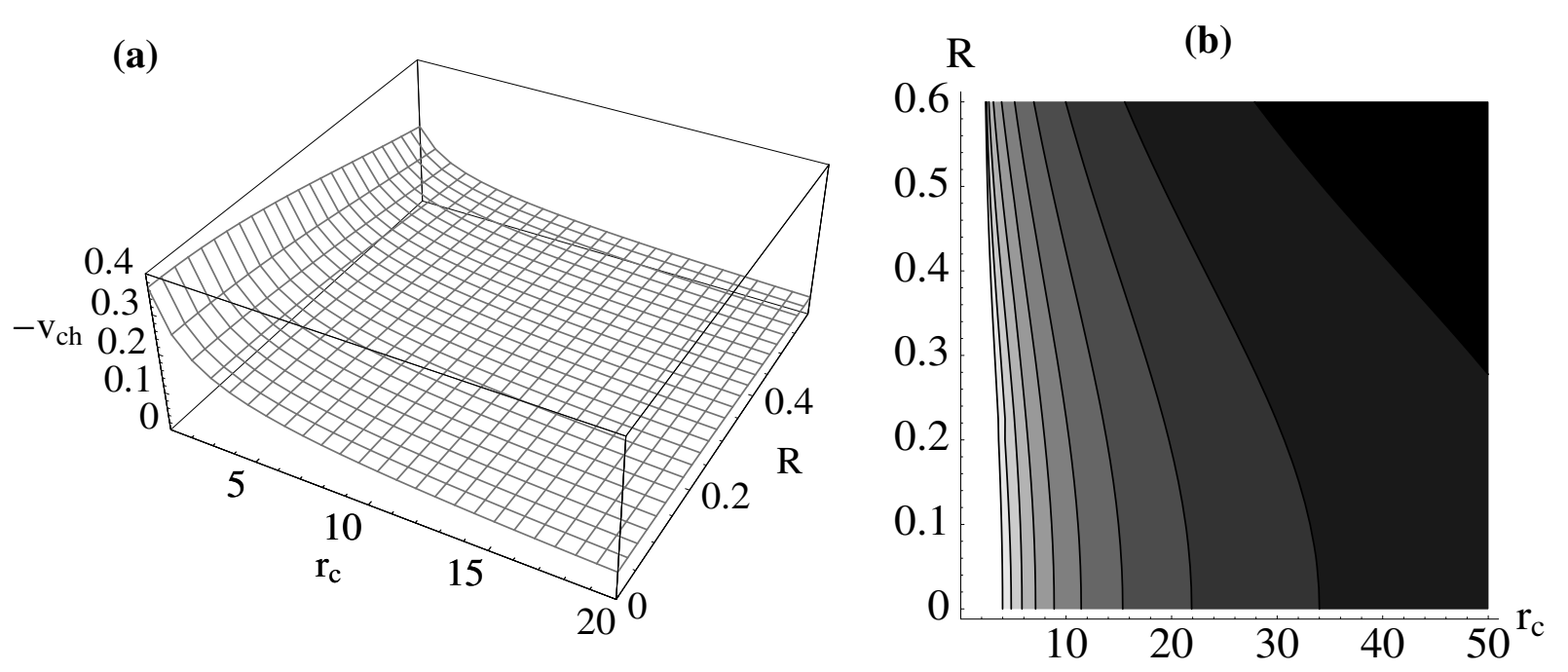

Figure 2.23: Variation of average chaotic velocity (equation (2.56) with a negative sign) as a function of its parameters namely cyclotron radius $r_{c}$ and the scatterer radius $R$, when $v_{0}=1, b=1$ and $a=1.2 b$. (b) The corresponding contour lines indicating equi-velocity lines (larger values are shown with lighter shades).

2.15). The figure also confirms that the current inside the system will be maximal if we allow a smaller radius for the semi-circular extended region.

A closer look at equations (2.40) and (2.56), shows that the value of maximum deviation from the horizontal are different. In the former model we have

$$
\theta=\arccos \left(1-\frac{b-R}{r_{c}}\right)
$$

whereas in the latter model we observe

$$
\theta=\arccos \left(1-\frac{b}{r_{c}}\right)
$$

These two values approach each other as $R \rightarrow 0$. Moreover, in this limit, the total phase space volume $\Omega_{\mathrm{uc}}$ approach each other. In this regime it is interesting to note that the magnitude of the average chaotic transport velocities (equations (2.40) and (2.56)) also approach each other. So here are two different models where the chaotic trajectories get scattered from the semi-circular regions in a different manner (in the former model they get scattered from a bulge whereas in the latter model they get scattered from a dip), but the average chaotic velocity nearly remains the same. To understand what made them 

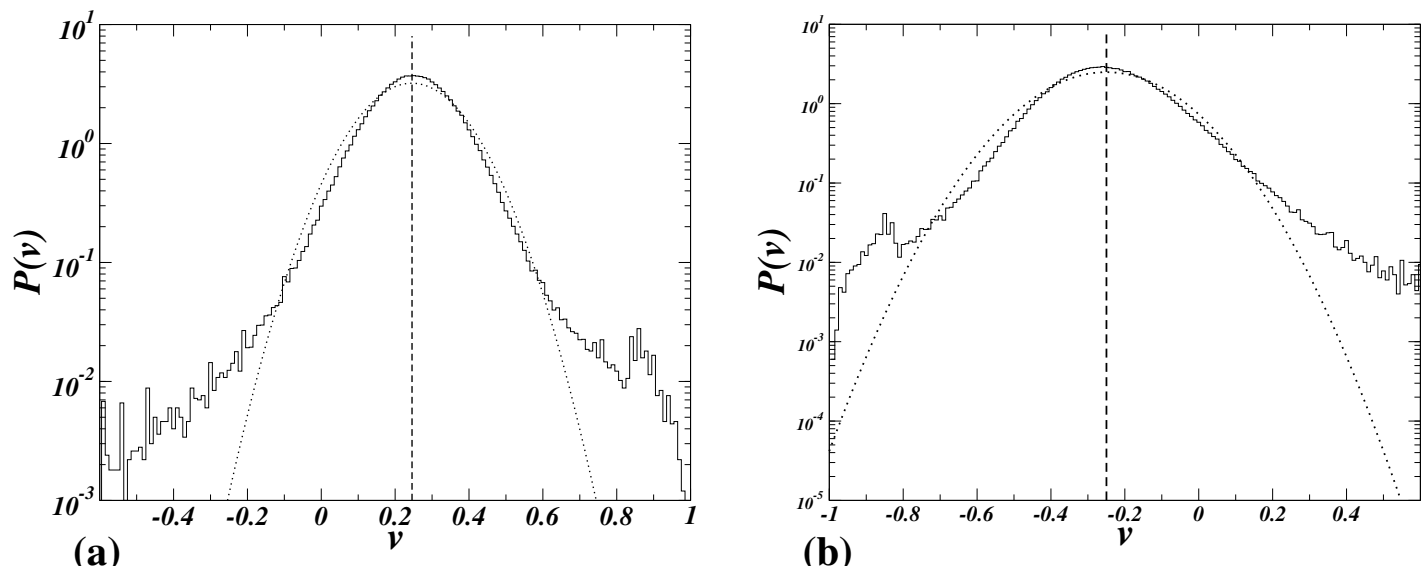

Figure 2.24: For small $R=0.01 b$, the dispersion of average chaotic velocities approaches a Gaussian with traces of large deviations for (a) figure 2.1 and (b) figure 2.20, $N=5 \times 10^{5}$ trajectories have been iterated for time $t=10^{3}$. The resulting distribution of average velocities is displayed as a histogram and compared to a Gaussian with same variance and mean (dotted line). The prediction of equations (2.40) and (2.56) for the average velocity is indicated by a vertical line in the respective distributions. Other parameters are same as in the construction of figure 2.20 .

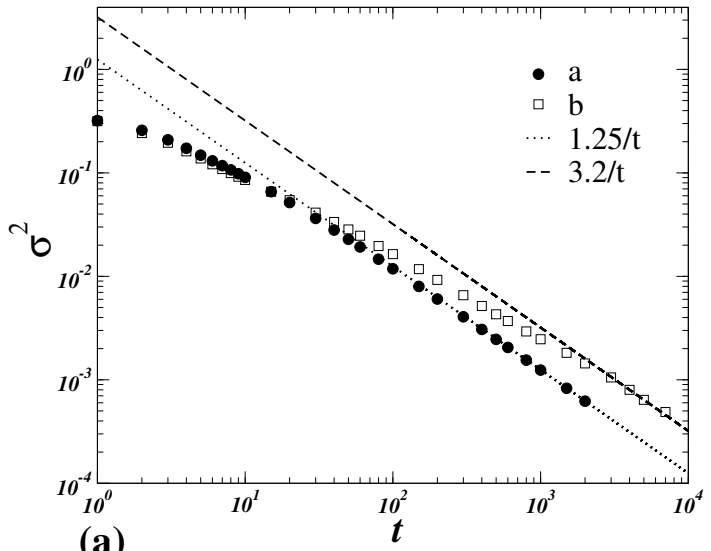

(a)

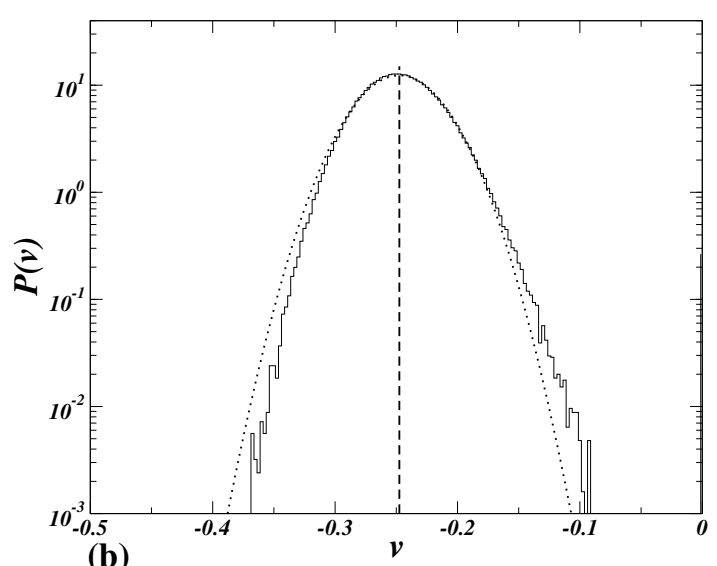

(b)

Figure 2.25: (a) Variation of the variance $\sigma^{2}$ of the velocity distribution as a function of time for our models of (a) figure 2.1 and (b) figure 2.20. The parameters used are same as in the construction of figure 2.20, The plot show asymptotic decay in time as $1 / t$ with the co-efficient providing the Diffusion constant for the corresponding system. (b) The distribution of velocities for small obstacle radius of $R=0.01 b$ and no separation between any two consecutive semi-circular regions for the model of figure 2.20 shows that the distribution is indeed a Gaussian. The dotted line show the corresponding Gaussian with same mean and variance. The analytical result of velocity from equation (2.56) is shown with a vertical dashed line. 
similar we simulate both the systems with a value of $R=0.01 b$ (keeping all other parameters same i.e, $b=1, a=1.2 b$ and $r_{c}=2 b$ ). The resulting distributions are shown in figure 2.24, As expected the magnitude of average chaotic transport velocity approach a common value. Also the distributions look more or less symmetric about their respective mean values. But there is a difference in the variance of the distributions. From this we may come to a conclusion that for same model parameters the variance of the distributions for our two waveguides vary at a different rate. To support this conclusion the variation of variance as a function of time has been plotted in figure 2.25 for the parameter values of $b=1, a=1.2 b, R=0.5 b$ and $r_{c}=2 b$. The figure shows the variation of variance $\sigma^{2}$ of the velocity distribution (refer to equation (2.47) ) as a function of time $t$. As can be seen, asymptotically the variance decreases as $1 / t$, with a constant coefficient providing the value of diffusion constant. But for smaller time scales the inverse power law of time fails. This is a consequence of correlations arising from trajectories sticking near to a periodic orbit (figure 2.17). Large deviations in the velocity distribution for both the systems, from the mean value, is also a consequence of these type of sticking trajectories. These correlations vanish if we allow the electron to roam around for a sufficient amount of time inside the channel or if we allow the parameters of the model such that the semi-circular regions make tangent to each other (such that the periodicity of the channel $a=2 R$ ) and do not allow gaps between them. This is confirmed from the distributions in figure 2.18 and figure 2.25 (b).

\subsection{Summary and Conclusion}

In this chapter we have introduced and studied two models with slight modifications in their geometries (in the former model we have a semicircular constriction whereas in the latter case we have a semicircular extension) which supports directed chaos in the presence of an appropriate magnetic field perpendicular to the plane of the waveguides. The most appealing feature of these models is that the phase space has, to a good approximation, a very simple structure which can be understood both intuitively and analytically. It consists of two invariant manifolds one chaotic and another regular. The latter is formed by trajectories skipping periodically along one perfectly straight wall of the channels. The other wall has semicircular distortions, periodic along the channels. This leads to backscattering and thus to a chaotic phase space component. Both the phase space structure and the broken time reversal symmetry are dependent on the presence of the transversal magnetic field acting on the electrons (in general they can be any kind of charged particle). For a very small magnetic field the phase space volume of the regular skipping orbits goes to zero and accordingly the chaotic transport is also very small. For stronger magnetic fields the phase space structure changes as more and more regular regions appear. Finally, for a very strong magnetic field, free cyclotron orbits (pinned trajectories) cover most of the phase space. In this regime $v_{\mathrm{ch}}$ approaches a non-zero constant, but at the same time the chaotic fraction of the phase space goes to zero.

For 1D driven Hamiltonian systems it has been shown that directed chaos may exist if the periodic 
driving is such that (i) all generalized time-reversal symmetries are broken [26] and (ii) the system has a mixed phase space in which regular and chaotic dynamics coexist [28]. Examples of this type have been investigated in a number of publications $[26,27,28,59,63]$, and closely related phenomena were realized experimentally with cold atoms in pulsed optical potentials [64, 65]. The driven Hamiltonian systems with directed chaos can generate directed transport in ensembles of particles which have initially a thermal distribution in phase space [26, 27, 28, 59, 64, 65]. They do so without an external bias force and under the influence of a potential which is periodic both in space and in time. This is very similar to the concept of stochastic ratchets (Brownian motors) $[1,3,6,6,7]$. Hence the term Hamiltonian ratchet is appropriate for the models investigated in $[26,27,28,59,64]$ and also for the atom-optics setup studied experimentally in [65]. However, magnetic billiards with directed chaos are no Hamiltonian ratchets and cannot generate directed transport from thermal ensembles. The reason is the conservation of energy. We have seen that the total transport from all regular and chaotic phase space components of an energy shell must vanish. Therefore directed transport in magnetic billiards requires control over the initial conditions beyond prescribing a certain distribution of energy values. For example, electrons could be placed selectively into the chaotic phase space component of our billiard chain if they enter the system from a lead which is attached to the distorted wall of the waveguide. In contrast we have addressed the question of directed chaos in a different situation which might be relevant in the context of solid-state physics. We have studied quasi-one-dimensional systems (billiard chains) in the presence of a transversal magnetic field breaking time-reversal symmetry and (ii) an asymmetry in the configuration of scatterers. Both these properties of the system provides a mixed phase space. In this way, we have considerably extended a preceeding study of directed chaos in magnetic billiards [66] because the specific geometry which we propose leads to a particularly simple phase space structure. This allows for detailed analytical calculations and provides sufficient insight in order to control the transport properties of our model with a few geometric parameters. For example, we obtain an explicit expression for the chaotic transport velocity. We can also understand in some detail the velocity dispersion of the chaotic trajectories. In known examples for directed chaos [26, 27, 28, 59] this dispersion was found to be non-Gaussian, with clear signatures of Lévy walks. In contrast, we identify a parameter that can be tuned such that the dispersion becomes essentially diffusive while $v_{\text {ch }} \neq 0$ remains constant. This might be a desirable feature in applications.

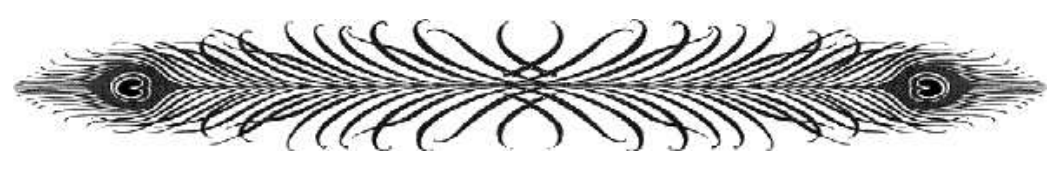





\section{Chapter 3}

\section{Transport in a Magnetic Billiard Chain with a Rough Boundary}

What is is what must be.

Gottfried Wilhelm von Leibnitz (1646-1716)

\subsection{Introduction}

In the previous chapter we have looked into two kinds of models, differed by a slight modifications of boundaries, which has supported the motion of chaotic trajectories into a single direction. The directionality of the chaotic orbits is a consequence of the motion of regular skipping orbits moving in the opposite direction. Also we have seen that as the radius of the semi-circular regions decreases to zero and simultaneously they make each other tangents at the contact between them (i.e., the separation between the semi-circular regions becomes zero), the velocity distribution approaches a Gaussian. In other words by tuning the radius of the semi-circular regions we can make the dispersion look like diffusive. We have seen, in this regime, how the detailed dynamics inside the lower boundary of the waveguide is irrelevant. This will hold true as long as the dynamics therein is chaotic. Therefore, the essential properties of our model won't get affected if we replace one of the boundary layers (say the lower boundary) by an idealized rough surface and describe the scattering from this wall probabilistically. In the next section (section 3.2) we follow this approach to obtain a better analytical understanding of such systems. For the sake of simplicity we will continue to refer the trajectories which hit the lower wall as chaotic although the dynamics is not deterministic anymore. This is justified since we have already explained (in the previous chapter) how random scattering may arise from a deterministic chaotic system in a certain limit. Nevertheless there are also other mechanisms such as thermal or quantum fluctuations which may lead to the same probabilistic model. Also we will discuss how in this case the 
dynamics can be replaced by the dynamics of a random walk. With the advancement of technologies we can construct any kind of random surfaces [67], and rough billiards are nowadays standard models for electron dynamics on mesoscopic scales [68, 69]. However, the system which we study here seems to be the first such model with a mixed regular-random phase space and is thus interesting in its own right. Therefore, the study of surfaces with corrugations deserves a special place.

\subsection{Billiard chain with a random corrugated surface}

The model we will be discussing is shown in figure 3.1. It consists of a 2D billiard channel with two parallel straight walls. A transversal magnetic field perpendicular to the plane of the surface of the billiard helps the electrons (charged billiard balls) to move in cyclotron orbits with radius $r_{c}$ inside the channel. The upper wall of the channel, being straight without corrugations, reflects the electrons specularly from its surface; whereas the lower wall can be thought of supporting closely packed obstructions (as in the previous chapter) or is disordered such that the electrons get reflected at random (thereby, determining the scattering at the lower wall probabilistically).

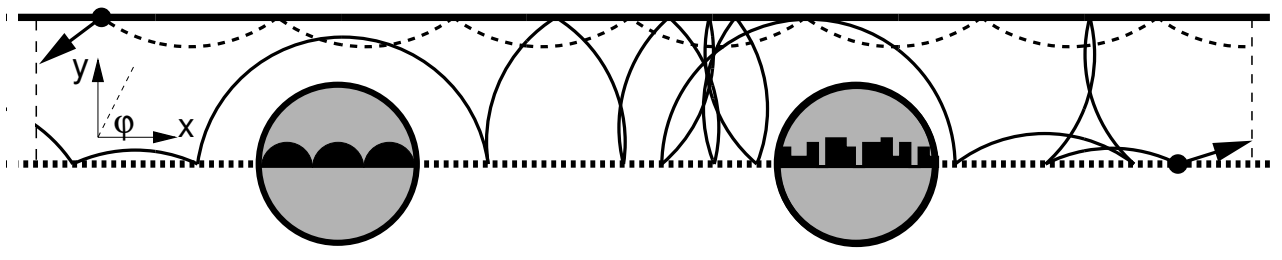

Figure 3.1: The billiard model for directed chaos with two different kind of surfaces one which reflect the electrons specularly and at the other surface the reflections are essentially random (see text for details). Some typical electron trajectories (dashed line : regular, full line : chaotic) are shown when the lower wall is perfectly rough $(\alpha=0)$ such that the angles before and after a reflection are completely uncorrelated.

In order to define this simplified model we must prescribe the probability for a trajectory to leave the lower wall at an angle $0 \leqslant \phi \leqslant \pi$. To keep consistency with the invariant measure $d \mu_{\|}$, equation (2.48), requires

$$
P(\phi)=\frac{1}{2} \sin \phi \quad(0 \leqslant \phi \leqslant \pi) .
$$

In terms of $c=\cos \phi$ this amounts to

$$
P(c)=\frac{1}{2} \quad(-1 \leq c \leq+1)
$$

for the probability density averaged over all chaotic trajectories. We can account for possible correlations between the segments of a trajectory by introducing a probability $\alpha$ for a specular reflection at the 
lower boundary. The probability density for $c_{n}$ is then

$$
P\left(c_{n}\right)=\alpha \delta\left(c_{n}-c_{n-1}\right)+\frac{1-\alpha}{2} .
$$

While for $\alpha=0$ there are no correlations between consecutive values of $c_{n}$

$$
\begin{aligned}
\left\langle c_{0} c_{n}\right\rangle & =\delta_{n, 0} \int_{-1}^{+1} d c P(c) c^{2} \\
& =\frac{\delta_{n, 0}}{3} \quad(\alpha=0),
\end{aligned}
$$

the possibility of specular reflections leads to exponentially decaying correlations

$$
\begin{aligned}
\left\langle c_{0} c_{n}\right\rangle & =\frac{1}{2} \int_{-1}^{1} d c_{0} \int_{-1}^{1} d c_{1} \ldots \int_{-1}^{1} d c_{n} \alpha \delta\left(c_{1}-c_{0}\right) \alpha \delta\left(c_{2}-c_{1}\right) \ldots \alpha \delta\left(c_{n}-c_{n-1}\right) c_{0} c_{n} \\
& =\frac{\alpha^{n}}{3} \quad(\alpha \neq 0) .
\end{aligned}
$$

For $\alpha=0$, we have shown a Poincaré map of the system in figure 3.2(b) and (c). As can be seen the Poincaré map shows that the chaotic part of the phase space is ergodic. Also since the upper wall of the channel does not contain any irregularities, the trajectories getting reflected only from this wall will be regular skipping trajectories. Therefore, we still can use the method of separation of regular and skipping trajectories and get an analytical expression for the average velocity of the chaotic trajectories. This will lead to an expression (2.40) or (2.56) but with the value of $R=0$ and any arbitrary $a$.

Alternatively, we note that, for each segment of a chaotic trajectory we can express the horizontal distance between its endpoints as

$$
\Delta x(\phi)=2 r_{c}\left(\sin \phi-\sin \phi^{\prime}\right)
$$

On the other hand the total length of the segment is

$$
\Delta l(\phi)=2 r_{c}\left(\phi-\phi^{\prime}\right) .
$$

Here $\phi$ and $\phi^{\prime}$ denote the angles of intersection with the lower and the upper wall of the channel, respectively (see figure 3.2(a)). In terms of the former, the latter is given by

$$
\phi^{\prime}=\left\{\begin{array}{ll}
\arccos \left(\cos \phi+\frac{b}{r_{c}}\right) & \left(\cos \phi \leqslant 1-\frac{b}{r_{c}}\right) \\
0 & \left(\cos \phi>1-\frac{b}{r_{c}}\right)
\end{array},\right.
$$


(a)

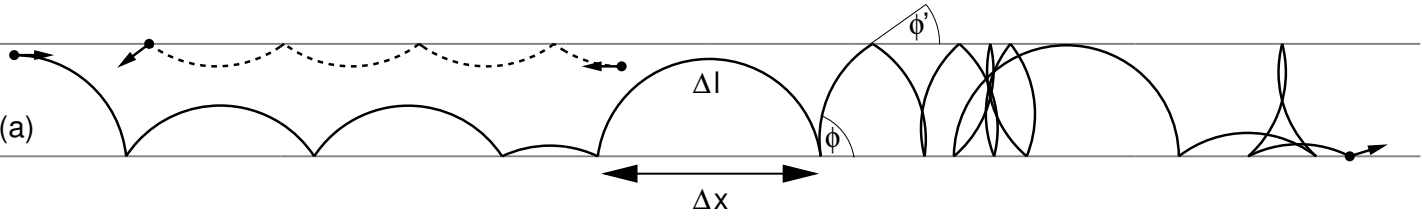

(b)

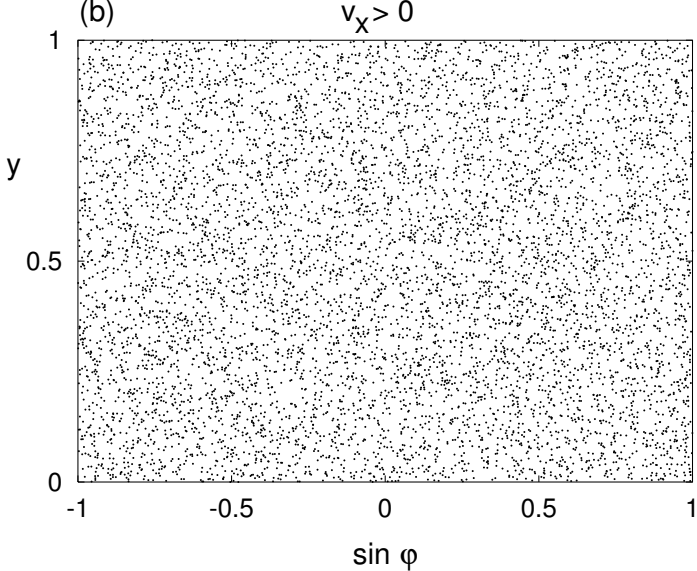

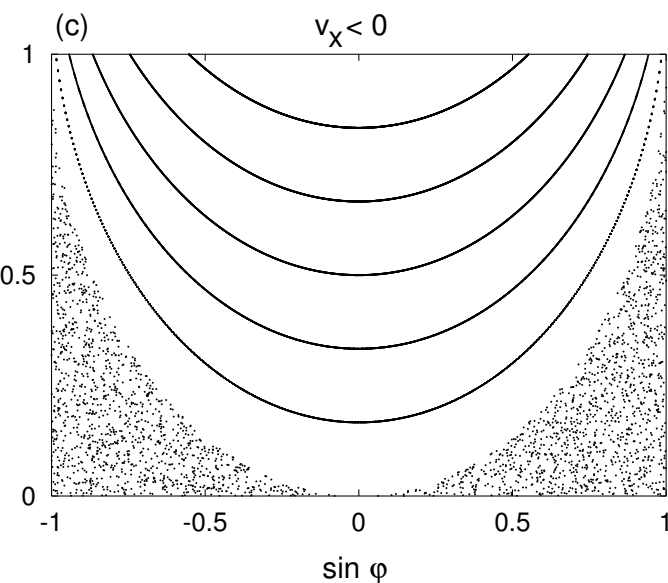

Figure 3.2: (a) Two trajectories (full line : chaotic, dashed line : regular) are shown for the model of figure 3.1. As the chaotic trajectory gets reflected from the rough wall of the channel it spans a horizontal distance $(\Delta x)$ during a time equal to the length of the corresponding section of the orbit $(\Delta l)$ (Since the the magnitude of velocity $v_{0}=1$ ). If $\phi$ denotes the initial angle of a trajectory at the lower wall of the channel then $\phi^{\prime}$ is the angle it will make at the upper wall of the channel. $\Delta x$ and $\Delta l$ for any section of a chaotic trajectory can be determined using these two variables $\left(\phi\right.$ and $\left.\phi^{\prime}\right)$ and the cyclotron radius $r_{c}$ (see text for details). Typical Poincaré maps for $x=0 \bmod 1$ when $r_{c}=b$ is shown for (b) $\cos \varphi>0$ and (c) $\cos \varphi<0$. A single chaotic trajectory fills both the regions of Poincaré map (shown here with dots) whereas the regular skipping orbits remain only to a single panel of the map (shown here like continuous lines in (c) for a set of them).

where the second line extends the definition of $\phi^{\prime}$ to those segments of a trajectory which do not reach the upper wall. Details of these calculations are given in Appendix A.

With the help of the angle $\phi$ we can provide an alternative parameterization of the chaotic component of phase space. Instead of $(x, y, \varphi)$ we can refer to one of its points by the coordinates $(x, \phi, l)$ (see figure 3.3). As mentioned above $\phi$ is the inclination angle of the segment at its initial point, and $0 \leq l \leq \Delta l(\phi)$ is the path length measured from there. Formally the transformations between $(x, \phi, l)$ and $(x, \varphi, y)$ are given by 


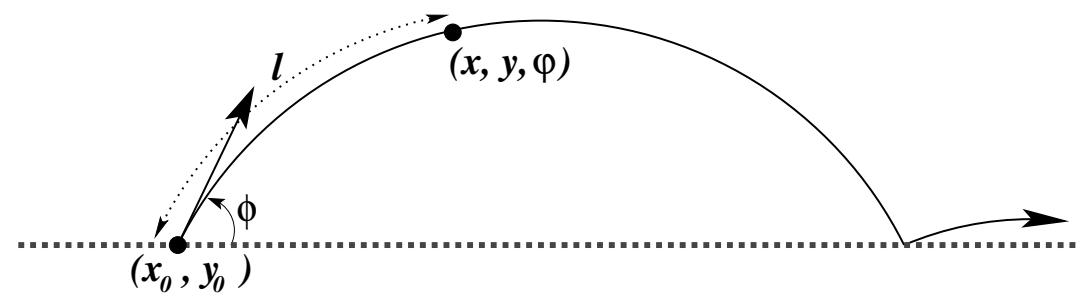

Figure 3.3: Chaotic phase space co-ordinate $(x, y, \varphi)$ can be represented in terms of $(x, \phi, l) . \phi$ is the inclination angle of the segment at the boundary and $l$ is the length of the point $(x, y, \varphi)$ from the reflection point at the boundary $\left(x_{0}, y_{0}=0\right)$. (See text for details of the transformation)

$$
\begin{aligned}
& \varphi(\phi, l)= \begin{cases}\phi-\frac{l}{r_{c}} & l \leqslant \frac{\Delta l(\phi)}{2} \\
-\varphi(\phi, \Delta l-l) & l>\frac{\Delta l(\phi)}{2}\end{cases} \\
& y(\phi, l)=r_{c} \cos \varphi(\phi, l)-r_{c} \cos \phi,
\end{aligned}
$$

where the second line of equation (3.10) expresses the reflection symmetry of the trajectory segments. The last equation (3.11) for the transformation can be derived from the fact that the point $\left(x_{0}, y_{0}=0, \phi\right)$ and $(x, y, \varphi)$ has a common center $\left(x_{c}, y_{c}\right)$ given by

$$
\left(\begin{array}{l}
x_{c} \\
y_{c}
\end{array}\right)=\left(\begin{array}{l}
x_{0}+r_{c} \sin \phi \\
y_{0}-r_{c} \cos \phi
\end{array}\right)=\left(\begin{array}{c}
x+r_{c} \sin \varphi \\
y-r_{c} \cos \varphi
\end{array}\right) \text {. }
$$

Then in terms of $\phi$ and $l$ we can express the chaotic trajectory contributions as

$$
\int_{\mathrm{ch}} d x d y d \varphi(\ldots)=\int_{\mathrm{ch}} d x d l d \phi\left|\frac{\partial(x, y, \varphi)}{\partial(x, l, \phi)}\right|(\ldots)
$$

where, $\frac{\partial(x, y, \varphi)}{\partial(x, l, \phi)}$ is the Jacobian matrix of the transformation from $(x, y, \varphi)$ to $(x, \phi, l)$ and $(\ldots)$ can be any measurable quantity of the chaotic phase space component (e.g., velocity $v_{x}$ ). Using the expressions given in equations (3.9)-(3.11) we can compute the determinant of this matrix to be

$$
\left|\frac{\partial(x, y, \varphi)}{\partial(x, l, \phi)}\right|=\sin \phi=2 P(\phi)
$$

Details of this calculation are given in Appendix B

Using these transformations we can compute arbitrary averages over the chaotic phase space com- 
ponents in terms of $\phi$ and $l$ as

$$
\begin{aligned}
\langle\ldots\rangle_{\mathrm{ch}} & =\Omega_{\mathrm{ch}}{ }^{-1} \int_{\mathrm{ch}} d \mu(\ldots)=\frac{\int_{\mathrm{ch}} d x d y d \varphi(\ldots)}{\int_{\mathrm{ch}} d x d y d \varphi} \\
& =\frac{\int_{0}^{\pi} d \phi P(\phi) \int_{0}^{\Delta l(\phi)} d l(\ldots)}{\int_{0}^{\pi} d \phi P(\phi) \int_{0}^{\Delta l(\phi)} d l} \\
& =\langle\Delta l\rangle_{\phi}^{-1} \int_{0}^{\pi} d \phi P(\phi) \int_{0}^{\Delta l(\phi)} d l(\ldots),
\end{aligned}
$$

where $\langle\ldots\rangle_{\phi}$ denotes the average of $(\ldots)$ over the probability density $P(\phi)$. Now we can use equation (3.15) to calculate the average chaotic transport velocity and the diffusion constant.

\subsubsection{Average chaotic transport velocity}

Using the above expression (3.15) we can provide an alternate derivation for the chaotic transport velocity in the case $R=0$. Using equation (3.7) we obtain

$$
\begin{aligned}
\int_{0}^{\Delta l(\phi)} d l \cos \varphi(\phi, l) & =\int_{0}^{\Delta l(\phi) / 2} d l \cos \left(\phi-\frac{l}{r}\right)+\int_{\Delta l(\phi) / 2}^{\Delta l(\phi)} d l \cos \left(-\phi+\frac{\Delta l(\phi)-l}{r}\right) \\
& =2 r_{c}\left[\sin \left(\frac{\Delta l(\phi)}{2 r_{c}}-\phi\right)+\sin \phi\right] \\
& =2 r_{c}\left(\sin \phi-\sin \phi^{\prime}\right)=\Delta x(\phi)
\end{aligned}
$$

which is obvious from the geometrical meaning of $\Delta x$ and $\Delta l$. With this identity we can apply equation (3.15) to equation (2.25) and obtain

$$
\begin{aligned}
v_{\mathrm{ch}} & =v_{0}\langle\Delta l\rangle_{\phi}^{-1} \int_{0}^{\pi} d \phi P(\phi) \int_{0}^{\Delta l(\phi)} d l \cos \varphi \\
& =v_{0}\langle\Delta l\rangle_{\phi}^{-1} \int_{0}^{\pi} d \phi P(\phi) \Delta x(\phi) \\
& =v_{0} \frac{\langle\Delta x\rangle_{\phi}}{\langle\Delta l\rangle_{\phi}}
\end{aligned}
$$

Equations (3.6) and (3.7) allow us to understand the chaotic transport as a random walk along the $x$-axis without any reference to the transversal motion. The discrete steps of this walk are $\Delta x(\phi)$ and the corresponding time increments are $\Delta l(\phi) / v_{0}$. Step $n$ is chosen according to the probability density 
in equation (3.3). From this random walk point of view the representation of average chaotic velocity, as in equation (3.17), is very intuitive: a long trajectory consists of $N \rightarrow \infty$ steps distributed according to $P(\phi)$. Therfore the distance between the end points of the trajectory is $\Delta X=N\langle\Delta x\rangle_{\phi}$ while the total time increment is $\Delta T=N\langle\Delta l\rangle_{\phi} / v_{0}$. The average longitudinal component of the velocity is then $\Delta X / \Delta T$ which is equivalent to equation (3.17). Explicit averaging over $\phi$ yields

$$
\langle\Delta x\rangle_{\phi}=r_{c} \int_{0}^{\pi} d \phi \sin \phi\left(\sin \phi-\sin \phi^{\prime}\right)=\frac{r_{c}}{2}(\theta-\sin \theta \cos \theta) .
$$

In agreement with the values of $\theta$, as in equations (2.59) and (2.60), we have substituted $\cos \theta=1-b / r_{c}$ to obtain this result. Similarly for average length of a segment we have

$$
\langle\Delta l\rangle_{\phi}=r_{c} \int_{0}^{\pi} d \phi \sin \phi\left(\phi-\phi^{\prime}\right)=r_{c}\left[\pi \frac{b}{r_{c}}-(\sin \theta-\theta \cos \theta)\right] .
$$

The explicit derivations of these results has been presented in Appendix $\mathrm{C}$. Combining equations (3.17)-(3.19) we find the expected result for the average chaotic transport velocity, namely equation (2.40) or equation (2.56) with $R=0$ (and arbitrary $a$ )

$$
v_{\mathrm{ch}}=\frac{v_{0}}{2} \frac{\theta-\sin \theta \cos \theta}{\left(\pi b / r_{c}\right)-(\sin \theta-\theta \cos \theta)} .
$$

We note that for equation (3.20) to be valid $\theta$ must satisfy certain conditions, namely

$$
\begin{aligned}
& |\cos \theta| \leqslant 1 \quad \Rightarrow \quad-1 \leqslant\left(1-\frac{b}{r_{c}}\right) \leqslant 1 \\
& \text { or } 2 r_{c} \geqslant b>0 \text {. }
\end{aligned}
$$

Therefore, if $2 r_{c}<b$ the equation (3.20) cannot be applied any more. Because in this regime a third phase space component of pinned cyclotron orbits appears in addition to regular skipping and chaotic trajectories. Hence, the phase space sum rule for $v_{\mathrm{ch}}$ must be modified to account for these free cyclotron orbits. In contrast we still can apply equation (3.17), but now we have $\phi^{\prime}=0$ for all $\phi$ since no chaotic trajectories will ever reach the upper wall of the waveguide. Therefore, we have

$$
\langle\Delta x\rangle_{\phi}=2 r_{c}\langle\sin \phi\rangle_{\phi}=2 r_{c} \frac{1}{2} \int_{-1}^{+1} d \cos \phi \sin \phi=\frac{\pi}{2} r_{c}
$$

and

$$
\langle\Delta l\rangle_{\phi}=2 r_{c}\langle\phi\rangle_{\phi}=\pi r_{c} .
$$




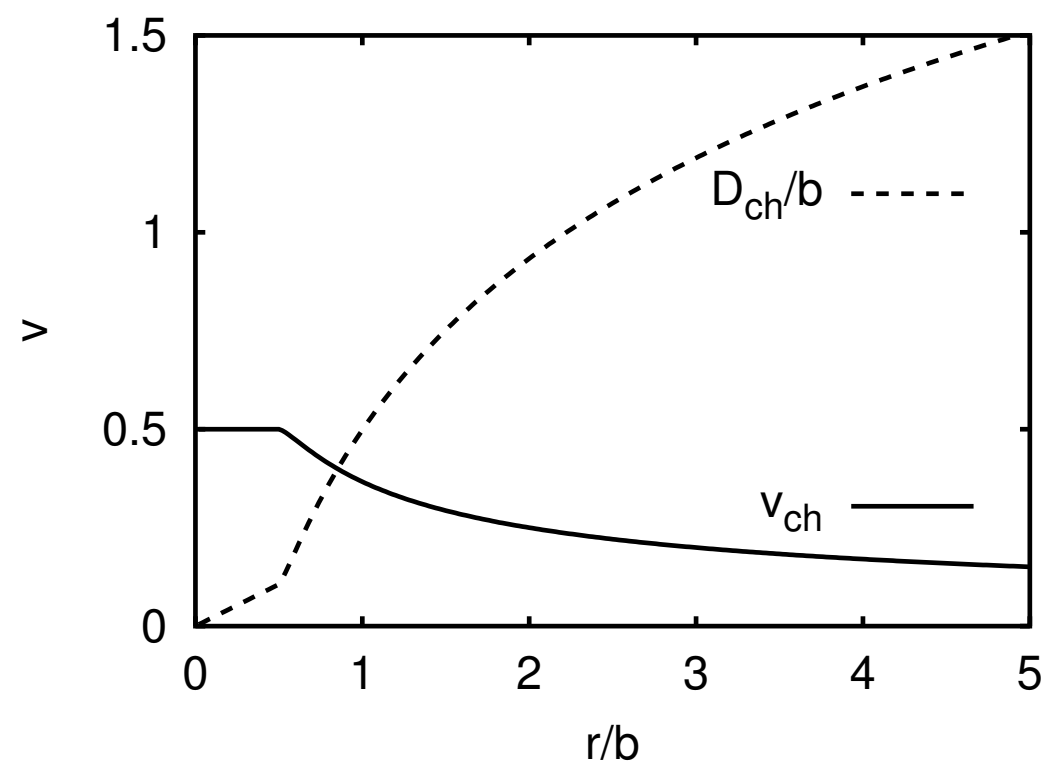

Figure 3.4: Dependence of the chaotic transport velocity (equation (3.20) ) and the diffusion constant (equation (3.29) ) on the cyclotron radius $r_{c}$ for random scattering at the lower wall $(\alpha=0)$.

Finally this gives

$$
v_{\mathrm{ch}}=\frac{v_{0}}{2} \quad\left(2 r_{c}<b\right) .
$$

Figure 3.4 shows the equation (3.20) as a function of the cyclotron radius along with the equation (3.24).

\subsubsection{Diffusion Constant}

Using the methods of the previous subsection we can also calculate the diffusion constant $D$ for our simplified model of a channel with a rough boundary. To start with we will use the well known Green-Kubo relation [70, 71, 72] which represents $D$ as an integral over the velocity autocorrelation function,

$$
D=\int_{-\infty}^{\infty} d t\left\langle\tilde{v}_{x}(0) \tilde{v}_{x}(t)\right\rangle
$$

Here $\tilde{v}_{x}(t)=v_{0} \cos \varphi(t)-v_{\mathrm{ch}}$ denotes the fluctuation of the transport velocity around its mean value and the average is taken over all the possible chaotic trajectories. If there are no correlations between different segments of a trajectory i.e., $\alpha=0$, then we can restrict the integral over time $t$ to the segment containing the phase space point $\left(\phi_{0}, l_{0}\right)$ at $t=0$ i.e., the interval over time $t$ will be restricted to

$$
-\frac{l_{0}}{v} \leqslant t \leqslant \frac{\Delta l\left(\phi_{0}\right)-l_{0}}{v_{0}} .
$$


Now we will use equation (3.15) to compute the averages

$$
\left.D_{0}=\frac{1}{\langle\Delta l\rangle_{\phi}} \int_{0}^{\pi} d \phi_{0} P\left(\phi_{0}\right) \int_{0}^{\Delta l\left(\phi_{0}\right)} d l_{0} \int_{-l_{0} / v_{0}}^{\left(\Delta l\left(\phi_{0}\right)-l_{0}\right) / v_{0}} d t\left[v_{0} \cos \varphi\left(\phi_{0}, l_{0}\right)-v_{\mathrm{ch}}\right]\left[v_{0} \cos \varphi(t)-v_{\mathrm{ch}}\right)\right]
$$

Substituting $l=l_{0}+v_{0} t$, we will obtain

$$
\left.D_{0}=\frac{1}{\langle\Delta l\rangle_{\phi}} \int_{0}^{\pi} d \phi_{0} P\left(\phi_{0}\right) \int_{0}^{\Delta l\left(\phi_{0}\right)} d l_{0} \int_{0}^{\Delta l\left(\phi_{0}\right)} d l v_{0}^{-1}\left[v_{0} \cos \varphi\left(\phi_{0}, l_{0}\right)-v_{\mathrm{ch}}\right]\left[v_{0} \cos \varphi\left(\phi_{0}, l\right)-v_{\mathrm{ch}}\right)\right]
$$

Here we will use the identity given in equation $(3.16)$ to find

$$
\begin{aligned}
D_{0} & =\frac{1}{\langle\Delta l\rangle_{\phi}} \int_{0}^{\pi} d \phi_{0} P\left(\phi_{0}\right) \frac{1}{v_{0}}\left[v_{0} \Delta x\left(\phi_{0}\right)-v_{\mathrm{ch}} \Delta l\left(\phi_{0}\right)\right]\left[v_{0} \Delta x\left(\phi_{0}\right)-v_{\mathrm{ch}} \Delta l\left(\phi_{0}\right)\right] \\
& =\frac{v_{0}}{\langle\Delta l\rangle_{\phi}} \int_{0}^{\pi} d \phi_{0} P\left(\phi_{0}\right)\left[\Delta x\left(\phi_{0}\right)-\frac{v_{\mathrm{ch}}}{v_{0}} \Delta l\left(\phi_{0}\right)\right]^{2} \\
& =v_{0} \frac{\left\langle\left[\Delta x-\left(v_{\mathrm{ch}} / v_{0}\right) \Delta l\right]^{2}\right\rangle_{\phi}}{\langle\Delta l\rangle_{\phi}}
\end{aligned}
$$

This result reduces the calculation of diffusion constant to a much simpler form which is numerically straight forward and is shown in figure 3.4. For strong magnetic field i.e., when $2 r_{c}<b$, we can compute the diffusion constant explicitly as

$$
\begin{aligned}
D_{0} & =v_{0} \frac{\int_{0}^{\pi} d \phi\left(2 r_{c} \sin \phi-\left(v_{\mathrm{ch}} / v_{0}\right) 2 r_{c} \phi\right)^{2} \sin \phi}{\int_{0}^{\pi} d \phi 2 r_{c} \phi \sin \phi} \\
& =2 r_{c} v_{0} \frac{1}{\pi}\left[\frac{4}{3}-\left(\pi^{2}-4\right)\left(\frac{v_{\mathrm{ch}}}{v_{0}}\right)^{2}-\pi^{2} \frac{v_{\mathrm{ch}}}{2 v_{0}}\right] \\
& =\frac{2 r_{c} v_{0}}{3 \pi} \quad\left(2 r_{c}<b\right),
\end{aligned}
$$

using the value of $v_{\mathrm{ch}}$ from equation (3.24). This reproduces the initial linear dependence of the diffusion constant on the cyclotron radius in figure 3.4

However if we allow correlations i.e., $\alpha \neq 0$ then complications arise as several segments of a trajectory can be identical. Accordingly, the section of the trajectory which is correlated with the initial point $\left(\phi_{0}, l_{0}\right)$ is longer. Thus the integration has to be extended over a longer range. To calculate this range we assume $\mu$ identical segments before the current one (where $\left(\phi_{0}, l_{0}\right)$ is located). Similarly we 


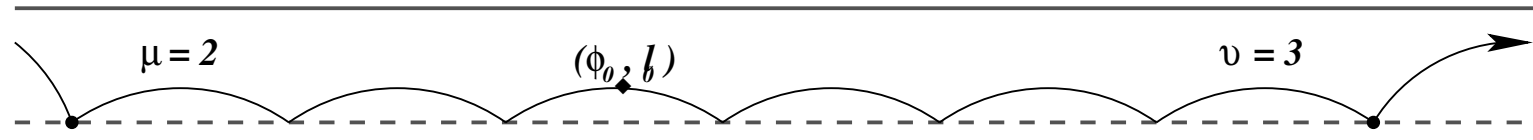

Figure 3.5: Starting from the current segment (where $\left(\phi_{0}, l_{0}\right)$ is located), we can have $\mu$ identical segments before the current one and $v$ identical segments after the current one. Here this is shown for $\mu=2$ and $v=3$. The random reflections are represented by a black dot. The current point $\left(\phi_{0}, l_{0}\right)$ is shown diamond shaped.

assume $v$ identical segments after the current one (see figure 3.5). Then the range of integration over $t$ in equation (3.27) will extend over

$$
-l_{0}-\mu \Delta l\left(\phi_{0}\right) \leqslant v_{0} t \leqslant \Delta l\left(\phi_{0}\right)-l_{0}+v \Delta l\left(\phi_{0}\right)
$$

i.e., the range of integration over $l$ in equation (3.28) will extend over

$$
-\mu \Delta l\left(\phi_{0}\right) \leqslant l \leqslant v \Delta l\left(\phi_{0}\right) .
$$

This increases the integral by a factor of $\mu+v+1$. In addition after all the identical segments on either side of the current segment there will be a random reflection. The probability for a random reflection before $\mu$ identical segments will be $\alpha^{\mu}(1-\alpha)$. Similarly the probability for a random reflection after the current one will be $\alpha^{v}(1-\alpha)$. Averaging with these probabilities over $\mu, v$ will yield

$$
\begin{aligned}
D_{\alpha} & =\sum_{\mu=0}^{\infty} \sum_{v=0}^{\infty} \alpha^{\mu}(1-\alpha) \alpha^{v}(1-\alpha)(\mu+v+1) D_{0} \\
& =\frac{1+\alpha}{1-\alpha} D_{0} .
\end{aligned}
$$

This is in perfect agreement with a correlated random walk [70]. The results are verified in figure 3.6 ,

We have already discussed that the billiard chain with a rough wall can be thought of as a random walk with step size $\Delta x$ in time $\Delta l / v_{0}$. The question arises if we can visualize the properties of our system using a random walk? Since our model shows a directed behavior along with diffusion of the particles (electron), it would be wise to think of a random walk model which also incorporates these properties. The answer lies with the biased random walk. In the next section, we will be discussing about this random walk and find out if it can represent some properties of our system. 

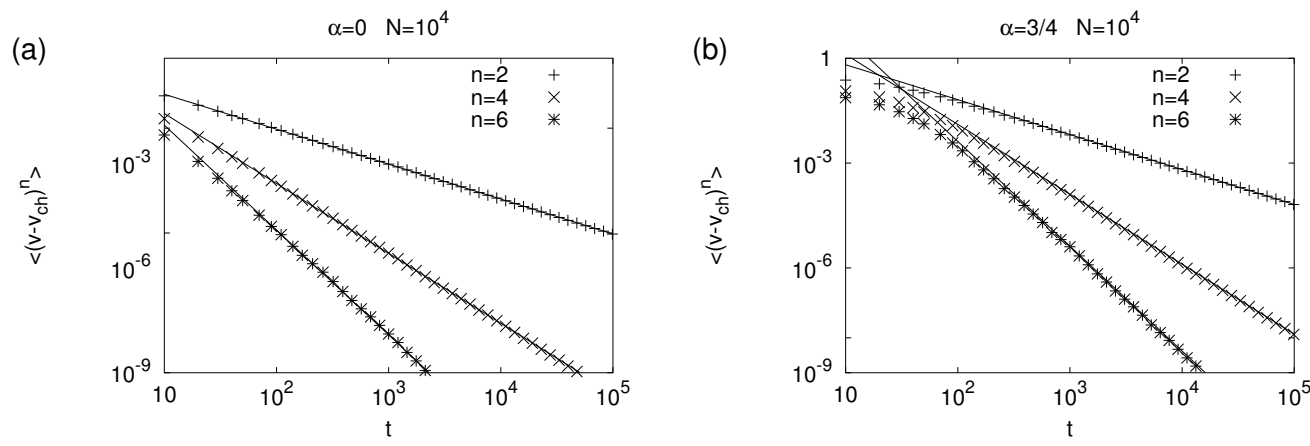

Figure 3.6: Some higher moments of the distribution of $N=10^{4}$ time-averaged chaotic transport velocities in the magnetic billiard with a rough lower wall and $r_{c}=2 b$. The probability of specular reflection is (a) $\alpha=0$ and (b) $\alpha=3 / 4$. Straight lines indicate diffusive spreading with the diffusion constant given by equations (3.30) and (3.33). The latter correctly predicts that $D_{\mathrm{ch}}$ is greater by a factor 7 in (b).

\subsection{Random Walk in One-Dimension}

Consider that the states of a system are the lattice points on a line with unit spacing $\ldots x-1, x, x+1 \ldots$ At times $N=0,1,2, \ldots$ a particle hops between neighboring sites on this one-dimensional chain (see figure 3.7). Let $\mathrm{p}$ be the hopping probability that a particle moves to the right direction and $q=1-p$ be the probability that the particle hops in the left direction. Let $P(x, N)$ be the total probability (occupational probability) that the particle is at site $x$ at the $N^{\text {th }}$ time step. Then the evolution of the occupation probability can be described using a master equation [73, 74]

$$
P(x, N+1)=p P(x-1, N)+q P(x+1, N)
$$

This states that the probability for the particle to be at site $x$ at time $N+1$ is simply $p$ times the probability of being at site $x-1$ at time $N$ (contribution of a step to the right) plus $q$ times the probability of being at site $x+1$ at time $N$ (step to the left). The case $p=q=1 / 2$ is the symmetric random walk, whereas for $p>q$ there is a uniform bias to the right and for $p<q$ a bias to the left. Now using the

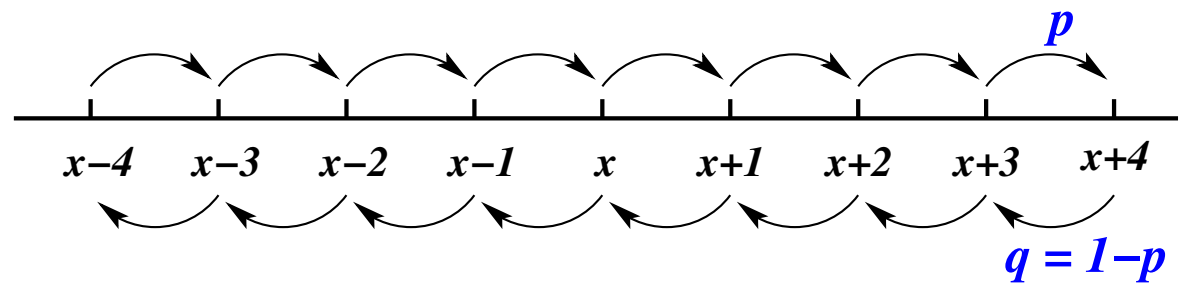

Figure 3.7: The hopping process for the one-dimensional nearest neighbor random walk defined by equation (3.34) 
master equation (3.34) we can obtain a differential equation when both space and time variables are continuous. We note that

$$
P(x, N+1)-(p+q) P(x, N)=p[P(x-1, N)-P(x, N)]+q[P(x+1, N)-P(x, N)],
$$

since $p+q=1$ for conservation of probability at any site. Here we have assumed that there is no absorption at the sites (which is satisfied for our system). Assuming a small bias to the probability in the positive direction of chaotic motion, i.e., $p=(1+\delta) / 2$ and $q=(1-\delta) / 2$, we get

$$
\begin{gathered}
P(x, N+1)-P(x, N)=\frac{1}{2}[P(x-1, N)-2 P(x, N)+P(x+1, N)] \\
-\frac{\delta}{2}[P(x+1, N)-P(x-1, N)]
\end{gathered}
$$

which can be rewritten in the following form

$$
\begin{gathered}
\frac{P(x, N+1)-P(x, N)}{[(N+1)-N] \tau}=\left\{\frac{P(x+1, N)-2 P(x, N)+P(x-1, N)}{[(x+1)-x] l[x-(x-1)] l}\right\} \frac{l^{2}}{2 \tau} \\
-\left\{\frac{[P(x+1, N)-P(x-1, N)]}{[(x+1)-(x-1)] l}\right\} \frac{\delta l}{\tau},
\end{gathered}
$$

where $l$ and $\tau$ are the units of the interval of the position and time respectively. We observe that the left hand side of equation (3.37) is a finite difference approximation to the first time derivative of $P(x, t)$ in the limit of $\tau \rightarrow 0$. Similarly the first term on the right hand side is the finite difference approximation to the second spatial derivative of $P(x, t)$ multiplied with $l^{2} /(2 \tau)$ and the second term on the right hand side can be recognized as the finite difference approximation to the first spatial derivative of $P(x, t)$ multiplied with $\delta l / \tau$. In the continuum limit we allow the spatial and the time units to approach 0 i.e., $\tau \rightarrow 0, l \rightarrow 0$ while the time $N$ and position $x$ approach infinity (so that $N \tau$ and $x l$ remain finite). At the same time we allow $\delta \rightarrow 0$ such that $l^{2} / \tau \rightarrow D$ and $\delta l / \tau \rightarrow v$. Also we replace the finite values $N \tau$ by time $t$ and $x l$ by position $x$, then we find that $P(x, t)$ satisfies the fundamental convection-diffusion equation[73],

$$
\frac{\partial P(x, t)}{\partial t}+v \frac{\partial P(x, t)}{\partial x}=\frac{D}{2} \frac{\partial^{2} P(x, t)}{\partial x^{2}} .
$$

for the particle concentration $P(x, t)$ which is the continuum analogue of the occupational probability of the random walk. Here $v=\delta l / \tau=(p-q) l / \tau$ defines the bias velocity and $l^{2} / \tau$ is the diffusion coefficient associated with the random walk. For the case of a symmetric random walk (when $p=q$ ), the probability distribution obeys the simpler diffusion equation

$$
\frac{\partial P(x, t)}{\partial t}=\frac{D}{2} \frac{\partial^{2} P(x, t)}{\partial x^{2}} .
$$


The reason for assuming $\delta \rightarrow 0$ is the following. In the convection-diffusion equation (3.38), the factor $v / D$ diverges as $1 / l$ in the continuum limit. Therefore the convective term $\partial P / \partial x$ dominates over the diffusive term $\partial^{2} P / \partial x^{2}$. So, if we want to construct a non pathological continuum limit, the bias $\delta$ must be proportional to $l$ as $l \rightarrow 0$. This will ensure that in our equation both the first and the second order spatial derivative terms will simultaneously remain finite. The equation (3.38) is also called the Biased Diffusion Equation. This is because, the probability is biased in a single direction which is sufficient to drive the motion into this direction (which is one of the features of our chaotic trajectories).

There are several ways for solving the equation (3.38). One of the methods have been described in Appendix $\mathrm{D}$. The solution for the probability distribution for the biased-diffusion equation will lead to

$$
P(x, t)=\frac{1}{\sqrt{2 \pi D t}} \exp \left[-\frac{(x-v t)^{2}}{2 D t}\right] .
$$

In terms of the velocity, with appropriate normalization, we will obtain

$$
P(x / t, t)=\sqrt{\frac{t}{2 \pi D}} \exp \left[-\frac{t(x / t-v)^{2}}{2 D}\right] .
$$

which agrees with equation (2.47), if we equate $\bar{v}_{t}$ with $x / t$ and $v$ with the average chaotic transport velocity $v_{\text {ch. }}$. Using this equation we can calculate the moments

$$
\begin{aligned}
& \left\langle\left(v_{t}-v_{\mathrm{ch}}\right)^{n}\right\rangle=\int_{-\infty}^{+\infty}\left(v_{t}-v_{\mathrm{ch}}\right)^{n} \sqrt{\frac{t}{2 \pi D}} \exp \left[-\frac{t\left(v_{t}-v_{\mathrm{ch}}\right)^{2}}{2 D}\right] d v_{t} \\
& =\frac{1}{\sqrt{\pi \beta}} \int_{-\infty}^{+\infty} \gamma^{n} \exp \left[-\frac{\gamma^{2}}{\beta}\right] d \gamma, \quad\left(\text { where } \beta=2 D / t, \gamma=v_{t}-v_{\mathrm{ch}}\right) \\
& =\left\{\begin{array}{cc}
\frac{\beta^{n / 2}}{\sqrt{\pi}} \int_{0}^{\infty} e^{-x} x^{(n-1) / 2} d x & (n \text { even })\left(\text { where } x=\gamma^{2} / \beta\right) \\
0 & \text { otherwise }
\end{array}\right. \\
& =\left\{\begin{array}{cc}
\frac{\beta^{n / 2}}{\sqrt{\pi}} \Gamma\left(\frac{n+1}{2}\right) & (n \text { even }) \\
0 & \text { otherwise }
\end{array} .\right.
\end{aligned}
$$

These results are shown in figure 3.8 for the distribution and in figure 3.9 for the moments of the velocity distribution. For these figures we have taken the value of cyclotron radius $r_{c}=4$ and $b=1$. As expected they very much agree with the distribution of velocities even for small time scales (as small as 10). However for much smaller values of time, the distribution shows a different behavior which do not agree with our expected results (see the distributions plotted in figure 3.8 (b) plotted for times $t=4,6,8$ and 10 with square, $\star,+$ and circle respectively). We observe that the distribution slowly approaches 
the expected Gaussian distribution of equation (3.41). The reason is the following. In deriving the Gaussian distribution in equation (3.41) we have assumed the initial condition $P(x, t=0)=\delta(x)$, which is not strictly true for the way we generate the initial conditions. The $N=10^{7}$ number of initial conditions were generated with equal probability of $x \in(0,1)$, but with the velocities (characterized by the inclination angle $\phi$ ) satisfying the probability distribution in equation (3.1). This is to ensure that our chaotic phase space has a uniform distribution. Moreover the dynamics of the electron is strictly not a continuous time random walk (the dynamics are represented by the values of $\Delta x(\phi)$ and $\Delta(\phi)$ ). Therefore, there is a time scale involved with our system during which the distribution approaches a Gaussian. This behavior is also reflected in the distribution of various moments of the distribution as shown in figure 3.9. However for larger time scales this effect becomes negligible and we get back the desired results.
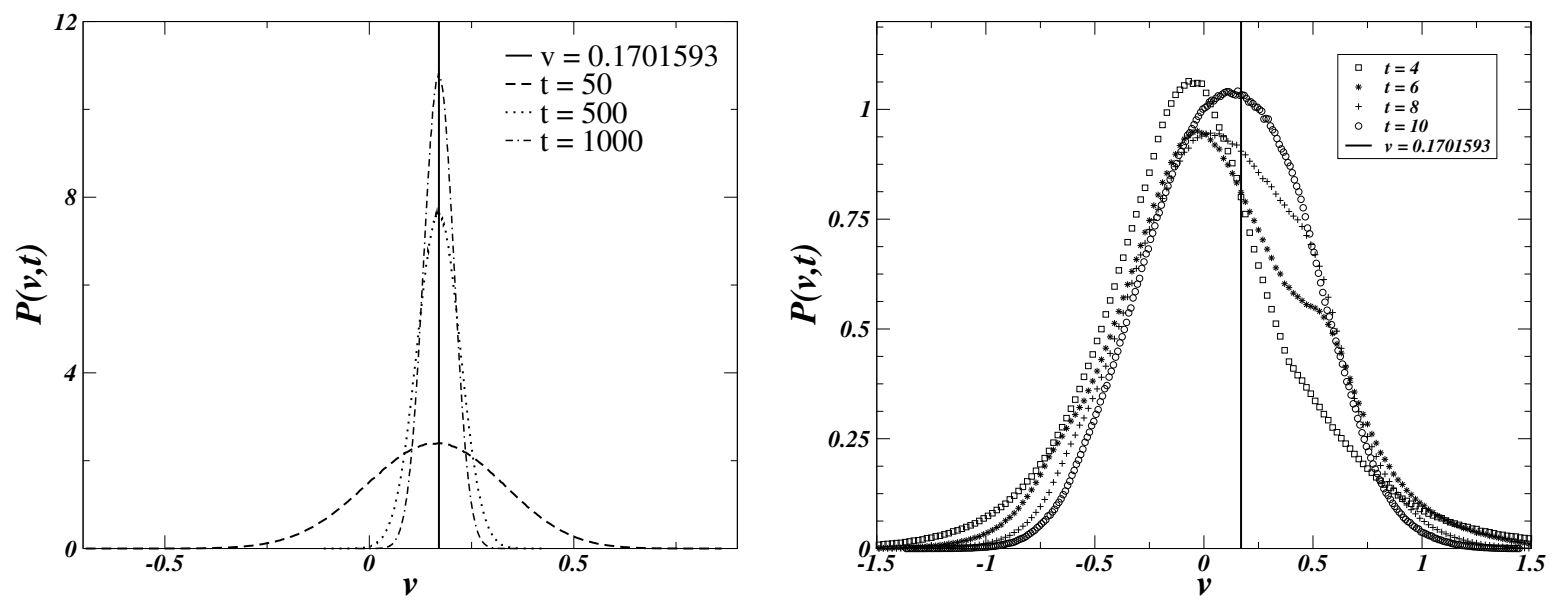

Figure 3.8: (a) The distribution of average velocities at various points of time $t=50,500$ and 1000 for the billiard chain with a rough boundary. The distribution very much agrees with the equation (3.41). The analytical value of average chaotic transport velocity as predicted from equation (3.20) has been shown with a vertical line. This predicted value nearly agrees with the statistical average value of $v_{\mathrm{ch}}=0.1695554$. (b) The small time distribution of velocity propagator at times $t=4$ (square), $6(\star)$, $8(+)$ and 10 (circle) for the billiard chain with a rough boundary. The analytical value of average chaotic transport velocity as predicted from equation (3.20) has been shown with a vertical line. For both of these $N=10^{7}$ number of phase space points have been chosen, with probability of inclination as in equation (3.1) along the line $y=0$ and $x \in(0,1)$, and propagated along the channel to find these distributions.

\subsection{Summary and Conclusion}

In the second chapter we showed that the magnetic billiard models support directed chaotic transport in the presence of an asymetrically placed semi-circular scatterers and a suitable perpendicular 


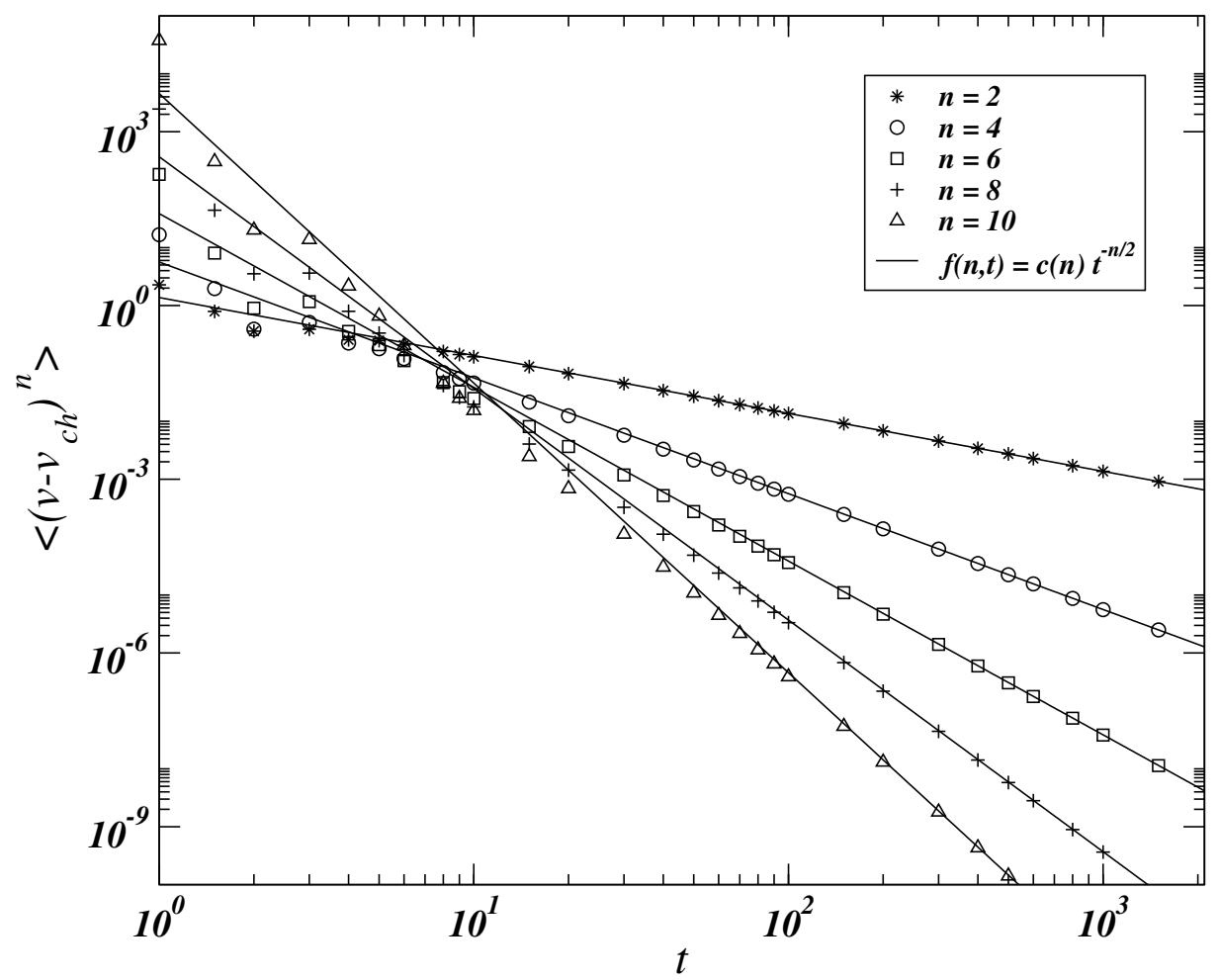

Figure 3.9: The distribution of various moments (of velocities) for the billiard chain with a rough boundary obtained numerically (symbols) has been compared with the analytical prediction (straight line) provided in equation (3.42). Clear deviations are seen away from the power law behavior for smaller time scales. These deviations are attributed to correlations resulting from our distribution of particles. Nevertheless for larger time scales the results very much agree with the results of a Gaussian distribution.

magnetic field breaking time reversal symmetry. However if the scatterer radius is very small the dynamics essentially becomes non-deterministic and they can be treated as random. This similarity with the random surfaces helped us extending the work of the previous chapter to a model of a billiard chain with a single rough surface (in the current chapter). In terms of the phase space and the chaotic transport velocity all of them show same kind of features, visually, ergodicity of the chaotic phase space and directedness of chaotic trajectories. Using a map of the chaotic orbits to the rough boundary we are able to show that the chaotic transport velocity can be calculated independently (without using a sum rule). However the important feature of the rough billiard model is the calculation of the diffusion constant using only the system parameters, which is impossible in the toy models of chapter two. Another consequence of the mapping is the calculation of average lateral displacements and the corresponding time probabilistically. This made the handling of the problem to a height where we are able to question the behavior of the system based on simple principles of random walk. In short we addressed a system 
where directed chaos leads to biased diffusion in case of an infinite system. In this regime of biased diffusion the chaotic dynamics can be approximated by a non-deterministic random walk in a billiard with a rough boundary, although our original model can be deterministic and has no disorder. 


\section{Chapter 4}

\section{Transmission in a Finite Rough Magnetic Billiard}

The chess-board is the world; the pieces are the phenomena of the universe; the rules of the game are what we call the laws of Nature. The player on the other side is hidden from us. We know that his play is always fair, and patient. But also we know, to our cost, that he never overlooks a mistake, or makes the smallest allowance for ignorance.

Thomas Henry Huxley (1825-95)

\subsection{Introduction}

According to Ohm's law, the resistance of a wire is proportional to its length. This is a straightforward consequence of the diffusive motion of electrons in the disordered potential of a normal material. However, unlike the time when $\mathrm{Ohm}$ arrived at his fundamental observation, conductors can be tailormade today with almost complete control over the microscopic structure. Therefore it is important to understand the consequences of non-diffusive electron dynamics on the electronic conductance or other transport properties. This question has been studied in much detail for semiconductor nanostructures in which the motion of electrons is ballistic rather than diffusive [75, 76, 77, 78, 79, 80]. In such systems disorder is negligible and consequently all transport properties are determined by the shape of the sample, as in a billiard model. For example, in the ideal case of a perfectly clean nanowire with parallel walls the resistance should be zero independent of the length, and indeed this remarkable prediction has been confirmed experimentally [78]. Besides the ballistic systems, the effects of anomalous diffusion on the electronic or thermal conduction properties has also attracted a lot of attention [69, 81, 82, 83].

In the previous chapter we studied the transport mechanism of the infinite magnetic billiard, but so far it could not provide us any information in case of an electronic setup of a finite sample (of length 
$L$ ) coupled to two electron reservoirs (see figure 4.1). In such systems the conductance play a major role. In our system, where there is coexistence of directed chaos (ballistic motion) along with diffusion (even in absence of any potential gradient), the conductance will depend on the system parameters, namely the width $b$, length $L$ and the external magnetic field characterized by the cyclotron radius $r_{c}$. For our purpose of calculations we assume a moderate magnetic field s.t., $r_{c}>b$. And for calculational

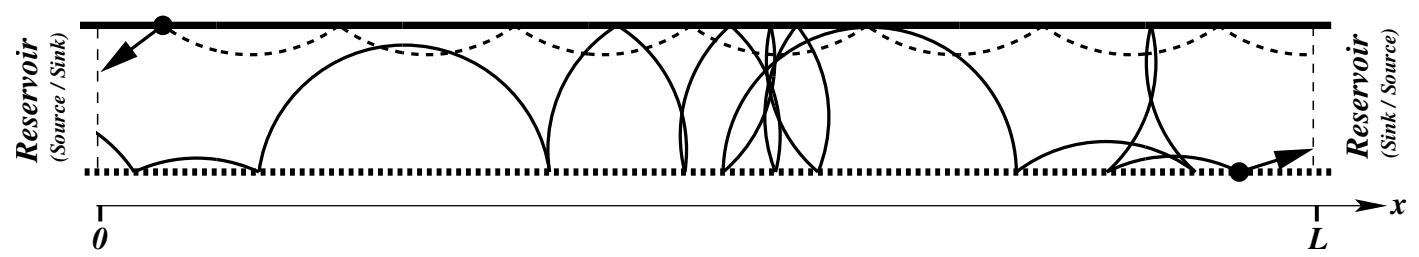

Figure 4.1: In case of an electronic setup, the finite sized magnetic billiard (of length $L$ ) is coupled to two electron reservoirs placed on either side of the waveguide. The electron reservoirs can act either as a source (providing electrons for conduction) or sink (draining out electron from the waveguide).

purpose we assume the value of $b=1$. In this case there are no pinned cyclotron orbits inside the bulk of the sample; and the phase space contains only two types of electron trajectories namely regular orbits skipping along the clean channel of the boundary and chaotic (or random) orbits which can get reflected from both the walls. On the average the chaotic orbits compensate the motion of the regular ones, making the system unbiased as a whole. However, we have seen that the chaotic transport velocity $\dot{x}$ of an electron is diffusive around its mean value. The average drift velocity can be calculated using a phase space sum rule or from the detailed dynamics of the chaotic trajectories by means of a mapping to the rough boundary. The value of the average chaotic transport velocity and also a diffusion coefficient are given in equations (3.20) and (3.29) respectively. We also are able to understand the dispersion of chaotic trajectories by replacing the microscopic dynamics of the model by using a Fokker-Planck Equation (FPE) for biased diffusion (see equation (3.38)). The question arises, if this much is sufficient to understand the conduction inside a finite channel? The answer clearly lies in understanding the origin of the electron transmission inside our billiard channel.

\subsection{Conductance in a finite Wire}

For nanoscale systems the electronic conductance is obtained within the framework of LandauerBüttiker formula [38, 75]. It is given in terms of the total transmission $T(L)$ as

$$
G(L)=\frac{2 e^{2}}{h} T(L)
$$

To derive this we consider a 1D wire connected to two large contacts by two leads as in figure 4.2. Lets 


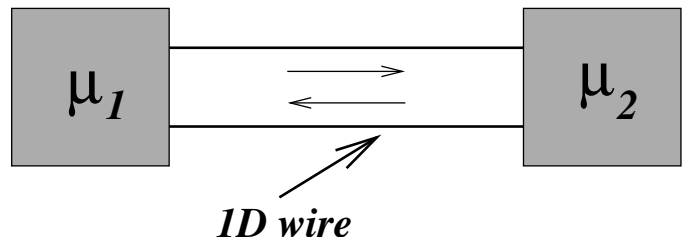

Figure 4.2: A 1D conductor connected to two reservoirs of chemical potentials $\mu_{1}$ and $\mu_{2}$.

assume that the number of occupied channels within the 1D conductor is $N$ and $T$ is the transmission probability. At zero temperature, the influx of electrons from reservoir 1 (at chemical potential $\mu_{1}$ ) to the reservoir 2 (at chemical potential $\mu_{2}$ ) is given by

$$
I_{1}^{+}=\frac{2 e}{h} N\left(\mu_{1}-\mu_{2}\right) .
$$

So the out-flux from reservoir 2 will be

$$
I_{2}^{+}=T I_{1}^{+}=\frac{2 e}{h} N T\left(\mu_{1}-\mu_{2}\right),
$$

and the rest of the flux is reflected back to reservoir 1 :

$$
I_{1}^{-}=\frac{2 e}{h} N(1-T)\left(\mu_{1}-\mu_{2}\right) .
$$

Therefore, the net current an any point in the device is

$$
I=I_{1}^{+}-I_{1}^{-}=I_{2}^{+}=T I_{1}^{+}=\frac{2 e}{h} N T\left(\mu_{1}-\mu_{2}\right) .
$$

Hence the conductance equal to

$$
G=\frac{I}{\left(\mu_{1}-\mu_{2}\right) /|e|}=\frac{2 e^{2}}{h} N T,
$$

providing the Landauer formula.

For our purpose we approximate the transmission semi-classically, $T \sim N t(L)$, where $t(L)$ denotes the total classical probability that the electron is transmitted through a sample of length $L$ if it enters the system at $x=0$ (i.e., from the left (see figure 4.1) with random initial conditions $P(y, \varphi)=\cos \varphi$ $(y \in[0,1], \varphi \in[-\pi / 2, \pi / 2]$ ) (see equation (2.8)). As per our direction of magnetic field these fall entirely within the chaotic component of phase space. Similarly, we define the probability $t^{\prime}(L)$ that an electron is transmitted if it enters at $x=L$ (i.e., from the right of the channel) with an analogous distribution but with $\varphi \in[\pi / 2,3 \pi / 2]$. If the system is in thermal equilibrium then the total transmission 
probability must be the same for the two distinct transport directions:

$$
t(L)=t^{\prime}(L) .
$$

If we assume that $t \neq t^{\prime}$, then it would mean there are absorbing points within our system or there is accumulation of particles in one of the reservoirs. But at thermal equilibrium this is not allowed. Also our system does not have any explicit potential centers inside it where absorption can occur. The direction independence of transmission probability can also be proved rigorously based on the fact that the scattering map of our Hamiltonian system is area preserving. Although relation (4.7) is not specific to directed chaos, it has profound consequences in the present model. While $t(L)$ is entirely due to chaotic trajectories whose properties are not immediately accessible, $t^{\prime}(L)$ can be decomposed into conditional probabilities for the respective components (namely regular and chaotic trajectories):

$$
t^{\prime}(L)=\mu_{\mathrm{reg}} t_{\mathrm{reg}}^{\prime}(L)+\mu_{\mathrm{ch}} t_{\mathrm{ch}}^{\prime}(L)
$$

where

$$
\mu_{\mathrm{reg}}=\frac{\int_{\mathrm{reg}} d y d \varphi \cos \varphi}{\int_{0}^{b} d y \int_{\pi / 2}^{3 \pi / 2} d \varphi \cos \varphi}=-\frac{1}{2 b} \int_{\mathrm{reg}} d y d \varphi \cos \varphi
$$

is the relative area of the regular component in the transversal Poincaré section (Figure 3.2(c)), and $\mu_{\mathrm{ch}}=1-\mu_{\mathrm{reg}}$ denotes the relative area of the chaotic trajectories in that section. From the dynamics of the regular trajectories, we can calculate $\mu_{\text {reg }}$ as

$$
\mu_{\mathrm{reg}}=-\frac{1}{2 b} \int_{0}^{b} d y \int_{\pi-\alpha(y)}^{\pi+\alpha(y)} \cos \varphi d \varphi .
$$

where $\pi \pm \alpha(y)$ denotes the maximum (minimum) deviation required for a trajectory starting in the phase space containing the point $y$ and still remaining regular. We already have calculated $\alpha$ in case of a billiard channel with a scatterer (refer to equation (2.35)). Therefore, for our purpose of a waveguide with a single rough wall we can assume $R=0$ and obtain

$$
\alpha(y)=\arccos \left(1-\frac{y}{r_{c}}\right) .
$$

Hence

$$
\begin{aligned}
\mu_{\mathrm{reg}} & =-\frac{1}{2 b} \int_{0}^{b} d y \int_{\pi-\alpha(y)}^{\pi+\alpha(y)} \cos \varphi d \varphi \\
& =\frac{1}{2} \frac{\theta-\cos \theta \sin \theta}{1-\cos \theta}
\end{aligned}
$$




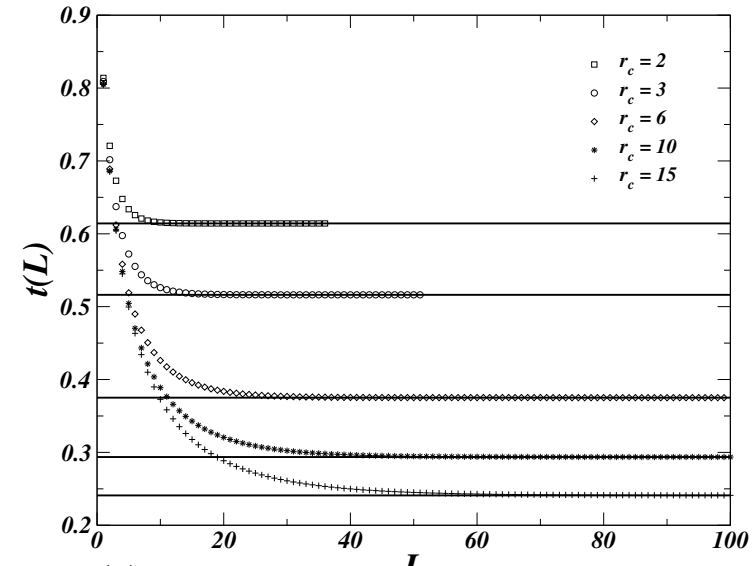

(a)

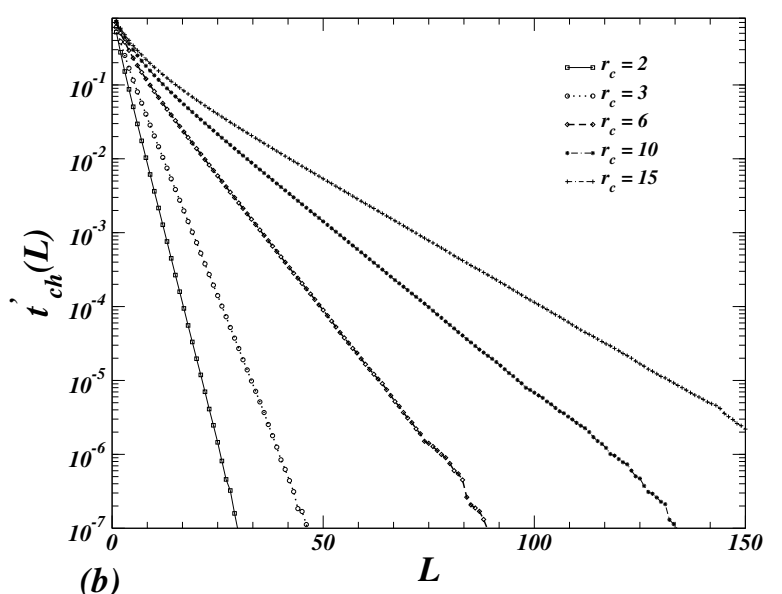

(b)

Figure 4.3: (a) The total transmission $t(L)$ and $(b)$ the transmission $t_{\mathrm{ch}}^{\prime}(L)$ resulting from chaotic trajectories as a function of the system length for various values of the cyclotron radius. The figure $(a)$ shows that the total transmission approaches a constant value predicted by equation (4.13) (plotted with a horizontal line) and the chaotic transmission $t_{\mathrm{ch}}^{\prime}(L)$ decreases exponentially with the increase in system length.

where $\theta=\arccos \left(1-b / r_{c}\right)$ provides the maximum deviation of a regular skipping orbit from the horizontal. See Appendix $C$ for details of this calculation.

For chaotic trajectories moving through arbitrarily long samples against the average chaotic flow, the time averaged velocity cannot converge to $v_{\mathrm{ch}}>0$. In other words they must need to be reflected in the positive direction. Therefore, it is clear that $t_{\mathrm{ch}}^{\prime}(L) \rightarrow 0$ for $L \rightarrow \infty$. Moreover, because of lack of backscattering along the regular trajectories, we must have $t_{\text {reg }}^{\prime}(L) \equiv 1$. Taken together these two facts yield a remarkable result which is illustrated in the figure 4.3 (a). For long systems, the probability that a chaotic trajectory transmits from $x=0$ to $x=L$ is given by the relative phase space area occupied by the counter moving regular skipping trajectories:

$$
t(L) \rightarrow \mu_{\text {reg }} \quad(L \rightarrow \infty)
$$

This result has been confirmed in figure 4.3 (a), where the horizontal line represents the value of $\mu_{\text {reg }}$. The goal now remains to understand quantitatively how $t_{\mathrm{ch}}^{\prime}(L)$ decays from $t_{\mathrm{ch}}^{\prime}(0)=1$ to zero as the length of the system increases. The reason for $t_{\mathrm{ch}}^{\prime}(0)=1$ is the following. If the system size decreases to zero (i.e., $L \rightarrow 0$ ), then almost all of the trajectories will transmit through the waveguide providing $t(L)=1$. Since $\mu_{\mathrm{reg}}+\mu_{\mathrm{ch}}=1$, we will get from equation (4.7) and (4.8) that $t_{\mathrm{ch}}^{\prime}(L) \rightarrow 1$ as $L \rightarrow 0$. The results of $t^{\prime}(L)$ using numerical simulations for a wide range of values of the cyclotron radius is shown in figure 4.3 (b). The data suggests an exponential behavior for long wires. In order to find out how the chaotic transmission $t_{\mathrm{ch}}^{\prime}(L)$ decreases exponentially to zero we replace the microscopic dynamics 
by the convection-diffusion equation. This has already been encountered in the previous chapter while describing the distribution of particle positions and/or velocities in case of a very long wire. However for an electron to be transmitted requires it to enter at one end of the channel and exit at the opposite end. Therefore, the details of the transmission probabilities require the calculation of exit probabilities in case of a biased random walk and the transmission probability based on them. We discuss them in the following section.

\subsubsection{Transmission Probabilities based on Biased Diffusion}

To describe the transmission in the framework of a FPE for biased diffusion (see equation 3.38), we assume absorbing boundary conditions at $x=0$ and at $x=L$. Then the probabilities to reach these exits from a point $0<x<L$ within the random walk (see figure 4.4) are [74, 84]

$$
\pi_{L}^{\prime}(x)=\frac{e^{-x / \lambda_{\mathrm{ch}}}-e^{-L / \lambda_{\mathrm{ch}}}}{1-e^{-L / \lambda_{\mathrm{ch}}}}
$$

and

$$
\pi_{L}(x)=1-\pi_{L}^{\prime}(x)=\frac{1-e^{-x / \lambda_{\mathrm{ch}}}}{1-e^{-L / \lambda_{\mathrm{ch}}}}
$$

respectively. The detailed calculations are provided in Appendix E. Here

$$
\lambda_{\mathrm{ch}}=D /\left(2 v_{\mathrm{ch}}\right)
$$

denotes the Peclet length of the biased diffusion process. Figure 4.5 shows the variation of this value with the ratio of the cyclotron radius to the width of the channel.

In order to contribute to the transmission from the right to the left end, for a wire of length $L$, a chaotic particle should first be transmitted through a segment of length $l<L$ and then starting from $x=L-l$, be absorbed at $x=0$ (figure 4.4). Based on this argument, we propose a recursion relation

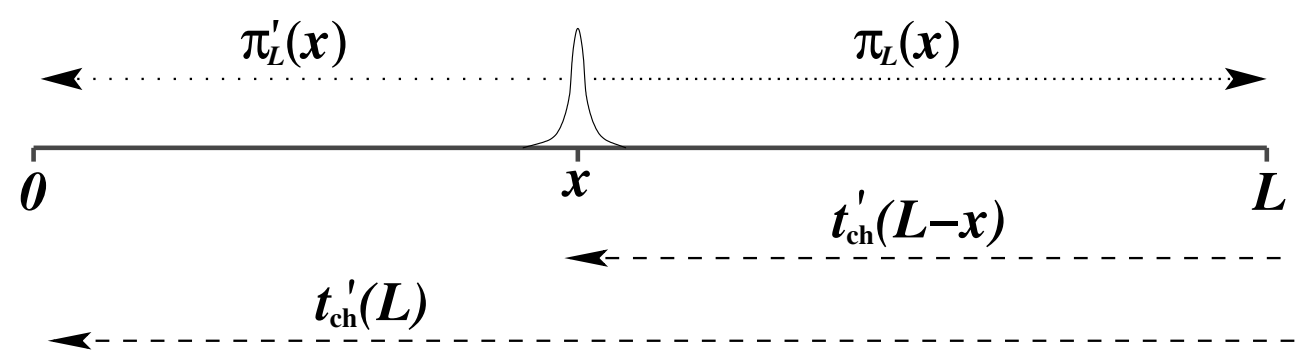

Figure 4.4: The transmission probability $t_{\mathrm{ch}}^{\prime}(L)$ can be related to the exit probability $\pi_{L}^{\prime}(x)$ of a random walk starting at $x$ (inside the system) by a simple recursion relation (4.17). 


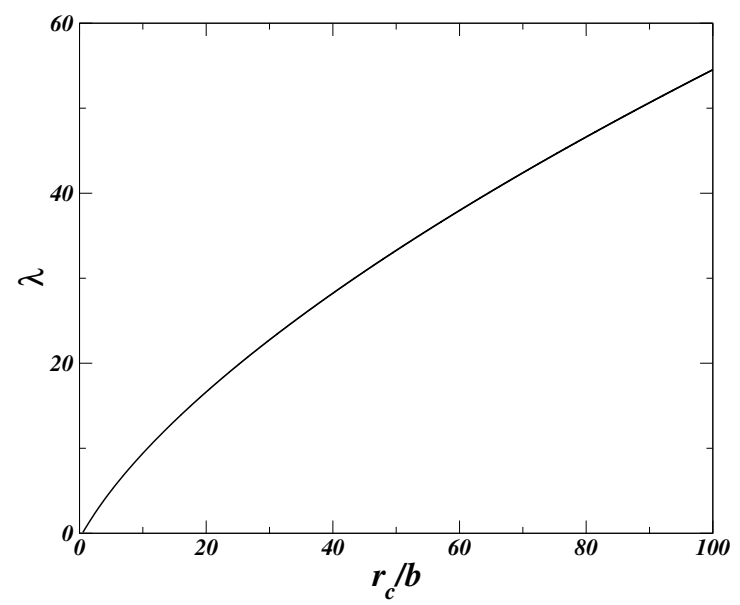

Figure 4.5: The variation of the Peclet length with the ratio of the cyclotron radius $r_{c}$ to the width of the channel $b$, when the magnetic field is not too strong $\left(r_{c} \geqslant 0.5 b\right)$.

for the chaotic transmission

$$
t_{\mathrm{ch}}^{\prime}(L)=t_{\mathrm{ch}}^{\prime}(l) \pi_{L}^{\prime}(L-l) .
$$

This condition can't be applied for any arbitrary value of $l$. For example, if we assume $l=0$, then $\pi_{L}^{\prime}(L)=0$ and simultaneously $t_{\mathrm{ch}}^{\prime}(L)$ vanishes. This is equivalent to, for a diffusing particle starting at one end of the absorbing boundaries the probability to reach the opposite end is identically zero. This is perfectly correct for the case of a random walk with absorbing boundaries. But for our sample this cannot be true. The reason for this limitation can be understood in the following way. In replacing the original dynamical system by a 1D FPE, we have discarded all the information about the momentum of the electrons. Strictly speaking, it is then impossible to define a transmission probability, since this will require entering the system with a given direction. Hence for equation (4.17) to be valid, the length scale for momentum correlations $\lambda_{\mathrm{ch}}$ should be negligible compared to the distance from the boundaries i.e.,

$$
\lambda_{\text {ch }} \ll l \quad \text { and } \quad \lambda_{\text {ch }} \ll L-l .
$$

Under this assumption we can write to leading order

$$
\pi_{L}^{\prime}(L-l)=\frac{e^{-(L-l) / \lambda_{\mathrm{ch}}}-e^{-L / \lambda_{\mathrm{ch}}}}{1-e^{-L / \lambda_{\mathrm{ch}}}} \simeq e^{-(L-l) / \lambda_{\mathrm{ch}}} .
$$

With this the recursion relation can be rewritten as

$$
t_{\mathrm{ch}}^{\prime}(L)=t_{\mathrm{ch}}^{\prime}(l) e^{-(L-l) / \lambda_{\mathrm{ch}}}
$$


which has a solution

$$
t_{\mathrm{ch}}^{\prime}(L)=c \exp \left(-L / \lambda_{\mathrm{ch}}\right)
$$

where $c$ is a constant. This reproduces the asymptotic decay of the transmission probability $t_{\mathrm{ch}}^{\prime}(L)$. It says that $t_{\mathrm{ch}}^{\prime}(L)$ decays exponentially with a characteristic length scale $\lambda_{\mathrm{ch}}$ when the system length is very large.

But this does not solve the problem! We still have to find an appropriate pre-factor. One of the very crude ways is to extrapolate the answer for $t_{\mathrm{ch}}^{\prime}(L)$ to the region of $L=0$. From equation (4.21), this will produce the answer $c=1$. However this value of $c$ does not reproduce the correct pre-factor. The other way is to extend the recursion relation (4.17) to $t(L)$, which will mean that for transmission from left to right end of a sample of length $L$ (which is exclusively via chaotic trajectories), the particle must transmit through a segment of length $l$ and then starting from $x=l$, be absorbed at $x=L$. This will provide us

$$
t(L)=t(l) \pi_{L}(l)
$$

This leads to

$$
\begin{aligned}
\mu_{\mathrm{reg}}+\mu_{\mathrm{ch}} c \exp \left(-L / \lambda_{\mathrm{ch}}\right) & =\left[\mu_{\mathrm{reg}}+\mu_{\mathrm{ch}} c e^{-l / \lambda_{\mathrm{ch}}}\right] \frac{1-e^{-l / \lambda_{\mathrm{ch}}}}{1-e^{-L / \lambda_{\mathrm{ch}}}} \\
& =\mu_{\mathrm{reg}}+\left(\mu_{\mathrm{ch}} c-\mu_{\mathrm{reg}}\right) e^{-l / \lambda_{\mathrm{ch}}}+\text { higher order terms. }
\end{aligned}
$$

If we compare both the sides of the equation then the leading order on the right hand side of the exponentials is $\exp \left(-l / \lambda_{\mathrm{ch}}\right)$. Clearly for the probability decaying exponentially with length $L$ cannot decrease with another length $l<L$. Therefore, the coefficient of this leading order must vanish. This provides us

$$
\mu_{\mathrm{ch}} c-\mu_{\mathrm{reg}}=0 \quad \text { or } \quad c=\frac{\mu_{\mathrm{reg}}}{\mu_{\mathrm{ch}}} .
$$

With this solution for the constant $c$ we can write

$$
t_{\mathrm{ch}}^{\prime}(L)=\frac{\mu_{\mathrm{reg}}}{\mu_{\mathrm{ch}}} \exp \left(-L / \lambda_{\mathrm{ch}}\right)
$$

In figure (4.6) we compare this result with the numerical calculations. We find that the solutions based on the FPE was able to predict the correct length scale $\lambda_{\mathrm{ch}}$ involved with the decay but it is not accurate enough to predict the pre-factor $c$ of the exponential decay (even $c=1$ is not a correct pre-factor). This is not a surprise. According to equation (4.21), a different pre-factor corresponds to an additive constant in the length $L$ of the system. This type of error is expected after discarding the correlation length of the momentum. Moreover, for short systems the behavior is clearly not exponential. This type of systematic errors could not be explained on the basis of the biased diffusion alone. Therefore, we conclude that a satisfactory theory cannot be based only on the basis of the FPE for biased diffusion. 


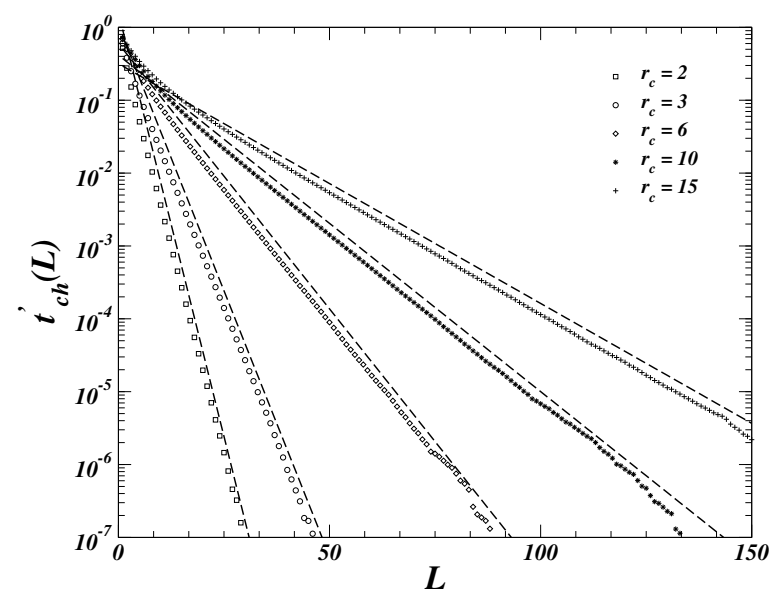

Figure 4.6: Comparison of numerical results for the chaotic transmission $t_{\mathrm{ch}}^{\prime}(L)$ (symbols) with the prediction of expression (4.25) (dashed line). The prediction was able to produce the length scale associated with the decay, but can not reproduce the correct pre-factor.

\subsubsection{Persistent Random Walk}

The simple random walk (FPE) provided us a valuable illustration as to how the transmission probabilities decay exponentially for large system lengths. Nevertheless it does not produce much details about the properties of the system e.g., it can not produce the small length scale behavior of transmission and also it is unable to produce a correct pre-factor for the asymptotic decay of transmissions. The reason being we do not have any information about the initial momentum of the system. Therefore, it seems we need to take care of this momentum in order to get a better description of our system. The answer lies with correlated (or persistent) random walk. In case of a persistent random walk (PRW) we add the memory of the previous step so that the walker continues in the same direction. In our model the chaotic trajectories always try to move in the direction of positive velocity (i.e., the motion is always left to right in other words from $x=0$ to the direction of $x=L$ ), whereas in the other direction the transmission probabilities of the chaotic trajectories decay to zero. This is equivalent to say that the walker always tries to move in a single direction (in our model this is from $x=0$ to $x=L$ ). This walk is referred to as a correlated random walk or a persistent random walk. The discrete time version of this model have been studied from a long time $[70,85,86,87]$. This model has been applied also to billiard models in order to understand deterministic diffusion [88]. The continuous version of the symmetric case (which leads to the telegrapher's equation [73, 89]) is the simplest model describing diffusion like processes involving not only the position of the particle but also a crude form of momentum (which is equivalent to memory). It has also been applied to problems of solid-state physics and thermodynamics [90, 91, 92].

For our purpose we initially discuss the asymmetric version of the correlated random walk and 
see how this can define some of the properties related to our system. Finally we will move to the scattering model describing this walk and explain the dynamics of transmission in our model. To understand the application of this model; instead of considering the dynamics over the entire length of the waveguide, we divide the full length of the wire into $n$ equal parts each of length $L_{1}$, so that $L=n L_{1}$. After each individual sections of length $k L_{1}, k \in 1,2, \ldots n$ the particle will have certain probability to get transmitted to the next segment of the wire. But we assume that the probabilities are direction dependent i.e., at any point of length of the system the particle will have probability for transmission $t_{k}$ provided the particle has come from left to right and $t_{k}^{\prime}$ if the particle has transmitted from right to left. Accordingly $r_{k}=1-t_{k}$ and $r_{k}^{\prime}=1-t_{k}^{\prime}$ are the corresponding reflection probabilities. This picture is equivalent to the persistent random walk (correlated random walk) with unequal reflection probabilities. Initially we describe this biased persistent random walk. Based on this we calculate an equivalent FPE and describe the expressions for velocity and the diffusion constant.

\subsection{Biased Persistent Random Walk (BPRW)}

As the biased random walk, this walk also takes place in 1D. However the rules of the movement are direction dependent. In general, the concerned particle (here an electron) does not change its direction at every collision, instead it retains the direction of motion with some probability. The rules for the movement of the particle is following [73, 93]:

- If the particle finds itself at position $x$ at time $t$, having arrived there from site $x-1$ it moves from site $x$ to $x+1$ with probability $p^{+}$or to site $x-1$ with probability $q^{+}=1-p^{+}$on the next move. However, if it has arrived from site $x+1$ then it moves to site $x-1$ with probability $p^{-}$or to site $x+1$ with probability $q^{-}=1-p^{-}$(see figure 4.7).

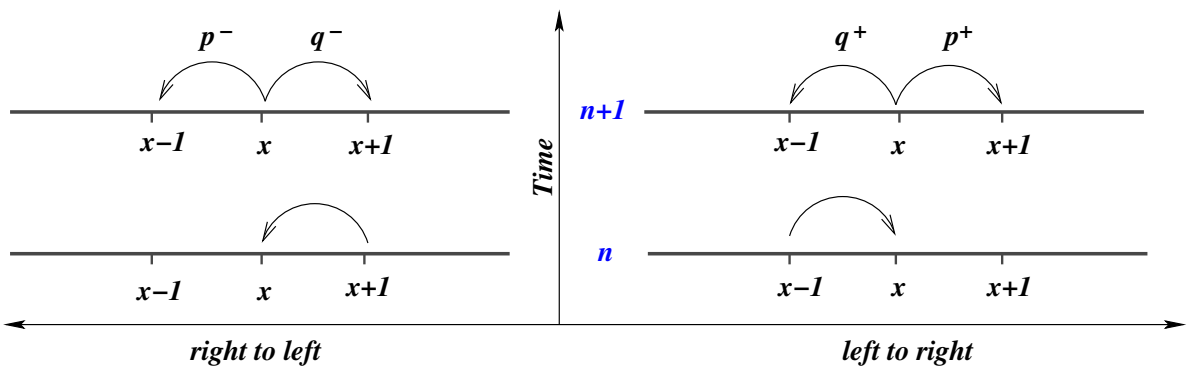

Figure 4.7: The asymmetric persistent walk (biased persistent random walk) takes place in 1D. A particle walks in the direction of motion of the previous step with probability $p^{+}$if it has arrived there from the left in the previous step and it reverses its direction with probability $q^{+}$. However if it has arrived from right then it continues to move in the left direction with probability $p^{-}$and reverses its movement with probability $q^{-}$. 


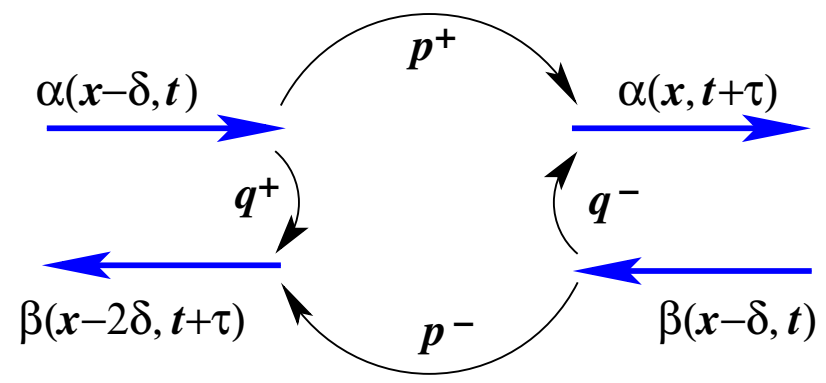

Figure 4.8: The scattering model describing the asymmetric persistent walk (biased persistent random walk) (See figure 4.7 for details in 1D).

In comparison to the history based game of Parrondo [18] (where the probability to win depends on the previous two steps), we can think BPRW as a history based game where the probability to win (movement in a single direction) only depends on the previous step.

We can combine these facts to make a scattering picture of this model. It is shown in figure 4.8 Using this scattering model, we can easily write a differential equation for BPRW in the following way. Let $\alpha(x, t)$ be the probability that the particle is at the position $x$ at time $t$ and arrived there from left, whereas $\beta(x, t)$ is the probability that the particle is at point $x$ at time $t$ and arrived there from right. Therefore, $\alpha(x, t)$ and $\beta(x, t)$ characterize right and left moving particles respectively. With a step size of length $\delta$ occurring at time intervals of length $\tau$, we can write the following coupled equations for the probabilities at the next time step

$$
\begin{aligned}
& \alpha(x, t+\tau)=p^{+} \alpha(x-\delta, t)+q^{-} \beta(x-\delta, t), \\
& \beta(x, t+\tau)=p^{-} \beta(x+\delta, t)+q^{+} \alpha(x+\delta, t) .
\end{aligned}
$$

Making use of a Taylor expansion in equations (4.26) and (4.27), and using the relation $p^{ \pm}=1-q^{ \pm}$, we will find to leading order

$$
\begin{aligned}
\alpha(x, t)+\tau \frac{\partial \alpha(x, t)}{\partial t} & =\left(1-q^{+}\right)\left[\alpha(x, t)-\delta \frac{\partial \alpha(x, t)}{\partial x}\right]+q^{-}\left[\beta(x, t)-\delta \frac{\partial \beta(x, t)}{\partial x}\right] \\
\Rightarrow \frac{\partial \alpha}{\partial t}+\frac{\delta}{\tau} \frac{\partial \alpha}{\partial x} & =-\frac{q^{+}}{\tau} \alpha+\frac{q^{-}}{\tau} \beta+\frac{q^{+} \delta}{\tau} \frac{\partial \alpha}{\partial x}-\frac{q^{-} \delta}{\tau} \frac{\partial \beta}{\partial x}
\end{aligned}
$$

Similarly from equation (4.27), we obtain

$$
\begin{aligned}
\beta(x, t)+\tau \frac{\partial \beta(x, t)}{\partial t} & =\left(1-q^{-}\right)\left[\beta(x, t)+\delta \frac{\partial \beta(x, t)}{\partial x}\right]+q^{+}\left[\alpha(x, t)+\delta \frac{\partial \alpha(x, t)}{\partial x}\right] \\
\Rightarrow \frac{\partial \beta}{\partial t}-\frac{\delta}{\tau} \frac{\partial \beta}{\partial x} & =-\frac{q^{-}}{\tau} \beta+\frac{q^{+}}{\tau} \alpha-\frac{q^{-} \delta}{\tau} \frac{\partial \beta}{\partial x}+\frac{q^{+} \delta}{\tau} \frac{\partial \alpha}{\partial x} .
\end{aligned}
$$


As the time step decreases to zero i.e., $\tau \rightarrow 0$, the probability of a persistent direction should tend to unity, whereas the probability of reversal should tend to zero. Therefore, for small $\tau$, we can write

$$
q^{+}=\lambda^{+} \tau \quad \text { and } \quad q^{-}=\lambda^{-} \tau
$$

$\lambda^{ \pm}$are the rates of reversals for the right (left) direction of transport, respectively. In the limit when $\delta$ and $\tau$ approaches zero such that $\delta / \tau \rightarrow v$, we can rewrite the above equations

$$
\begin{aligned}
& \frac{\partial \alpha(x, t)}{\partial t}+v \frac{\partial \alpha(x, t)}{\partial x}=-\lambda^{+} \alpha(x, t)+\lambda^{+} \beta(x, t), \\
& \frac{\partial \beta(x, t)}{\partial t}-v \frac{\partial \beta(x, t)}{\partial x}=\lambda^{+} \alpha(x, t)-\lambda^{-} \beta(x, t) .
\end{aligned}
$$

Adding and subtracting these two equations yields,

$$
\begin{aligned}
& \frac{\partial[\alpha(x, t)+\beta(x, t)]}{\partial t}+v \frac{\partial[\alpha(x, t)-\beta(x, t)]}{\partial x}=0 \\
& \frac{\partial[\alpha(x, t)-\beta(x, t)]}{\partial t}+v \frac{\partial[\alpha(x, t)+\beta(x, t)]}{\partial x}=-2\left[\lambda^{+} \alpha(x, t)-\lambda^{-} \beta(x, t)\right] .
\end{aligned}
$$

Substituting $P(x, t)=\alpha(x, t)+\beta(x, t)$ and $Q(x, t)=\alpha(x, t)-\beta(x, t)$, we find that they satisfy

$$
\begin{aligned}
& \frac{\partial P(x, t)}{\partial t}+v \frac{\partial Q(x, t)}{\partial x}=0 \\
& \frac{\partial Q(x, t)}{\partial t}+v \frac{\partial P(x, t)}{\partial x}=\left(\lambda^{-}-\lambda^{+}\right) P(x, t)-\left(\lambda^{+}+\lambda^{-}\right) Q(x, t) .
\end{aligned}
$$

As the next step we differentiate equation (4.34) w.r.t. time $t$ and equation (4.35) w.r.t. position $x$. This yields

$$
\begin{aligned}
& \frac{\partial^{2} P(x, t)}{\partial t^{2}}+v \frac{\partial}{\partial t} \frac{\partial Q(x, t)}{\partial x}=0 \\
& \frac{\partial}{\partial x} \frac{\partial Q(x, t)}{\partial t}+v \frac{\partial^{2} P(x, t)}{\partial x^{2}}=\left(\lambda^{-}-\lambda^{+}\right) \frac{\partial P(x, t)}{\partial x}-\left(\lambda^{+}+\lambda^{-}\right) \frac{\partial Q(x, t)}{\partial x} .
\end{aligned}
$$

As the final step we multiply equation (4.37) with $v$ and subtract relation (4.37) from equation (4.36) and make use of the relation (4.34) between $Q$ and $P$, to find $P$ satisfying

$$
\frac{\partial^{2} P(x, t)}{\partial t^{2}}+\left(\lambda^{+}+\lambda^{-}\right) \frac{\partial P}{\partial t}=v^{2} \frac{\partial^{2} P(x, t)}{\partial x^{2}}+v\left(\lambda^{+}-\lambda^{-}\right) \frac{\partial P(x, t)}{\partial x} .
$$


In the limit when $\lambda^{+}=\lambda^{-}=\lambda$, i.e., when the rate of reversals are direction independent, we get the symmetric correlated random walk or simply the persistent random walk, whose probability density satisfies the telegrapher's equation

$$
\frac{\partial^{2} P(x, t)}{\partial t^{2}}+2 \lambda \frac{\partial P(x, t)}{\partial t}=v^{2} \frac{\partial^{2} P(x, t)}{\partial x^{2}} .
$$

where we have assumed

$$
P(x, t)=\alpha(x, t)+\beta(x, t)
$$

It denotes the probability density function associated with the position $x$ at time $t$ without any mention of direction in which the particle is moving. The telegrapher's equation was originally derived by W. Thomson (Lord Kelvin) to describe the propagation of telegraph signals in underwater cables [73].

Looking back into equation (4.38), it is clearly different to the ordinary biased random walk leading to a biased diffusion equation (refer to equation (3.38)). In the biased persistent random walk, the difference in the reversal rates, $\lambda^{+}-\lambda^{-}$provides the drift term (i.e., the first order $x$-derivative term). However both the equations can be related. To relate this equation to the FPE, we assume that the time $t$ and spatial co-ordinate $x$ are very large i.e., $x \rightarrow \infty, t \rightarrow \infty$ and are of the same order of magnitude. In this limit we assume

$$
t=\frac{\tilde{t}}{\epsilon} \quad \text { and } \quad x=\frac{\tilde{x}}{\epsilon} \quad \text { where } \quad 0<\epsilon \ll 1 .
$$

Substituting back into the equation (4.38), gives rise to

$$
\epsilon^{2} \frac{\partial^{2} P}{\partial \tilde{t}^{2}}+\left(\lambda^{+}+\lambda^{-}\right) \epsilon \frac{\partial P}{\partial \tilde{t}}=v^{2} \epsilon^{2} \frac{\partial^{2} P}{\partial \tilde{x}^{2}}+v\left(\lambda^{+}-\lambda^{-}\right) \epsilon \frac{\partial P}{\partial \tilde{x}} .
$$

For very small $\epsilon$ this equation can be approximated to

$$
\frac{\partial P}{\partial t}=v\left(\frac{\lambda^{+}-\lambda^{-}}{\lambda^{+}+\lambda^{-}}\right) \frac{\partial P}{\partial x}=v\left(\frac{q^{+}-q^{-}}{q^{+}+q^{-}}\right) \frac{\partial P}{\partial x}
$$

Multiplying with $x$ and integrating over the range $x:\{-\infty, \infty\}$ yields

$$
\frac{d\langle x\rangle}{d t}=-v_{e f f} \int_{-\infty}^{\infty} x \frac{\partial P}{\partial x} d x
$$

where, we have set

$$
\begin{array}{r}
v_{e f f}=v\left(\frac{q^{-}-q^{+}}{q^{+}+q^{-}}\right)=v\left(\frac{\lambda^{-}-\lambda^{+}}{\lambda^{+}+\lambda^{-}}\right) \\
\text {and }\langle x\rangle=\int_{-\infty}^{\infty} x P(x, t) d x
\end{array}
$$


In order to evaluate the integral in equation (4.44), we assume that the probability density function $P(x, t)$ vanishes sufficiently rapidly at infinity and integrate the equation by parts

$$
\int_{-\infty}^{+\infty} x \frac{\partial P}{\partial x} d x=\left.x P(x, t)\right|_{-\infty} ^{+\infty}-\int_{-\infty}^{+\infty} P(x, t) d x=-1
$$

Therefore,

$$
\frac{d\langle x\rangle}{d t}=v_{e f f}
$$

Now integrating with time in the range $t:\{0, t\}$ we obtain

$$
\left.\langle x\rangle\right|_{t}-\left.\langle x\rangle\right|_{0}=v_{e f f} t
$$

As in the case of FPE approach, we assume a delta function distribution for the particle at time $t=$ $0, P(x, 0)=\delta(x)$. The property of this delta function at $t=0$ says that $\left.\langle x\rangle\right|_{0}=0$. This provides the moment $\langle x\rangle$ to be

$$
\langle x\rangle=v_{e f f} t=v \frac{q^{-}-q^{+}}{q^{+}+q^{-}} t .
$$

In other words the drift velocity of the particle in case of BPRW depends on the difference of the reverse probabilities between the forward $q^{-}$and the backward $q^{+}$directions. This is completely different than the FPE approach. Within the FPE a similar calculation will show that

$$
\langle x\rangle=v t .
$$

So, the effect of unequal scattering in both the directions of transport reduces the value of velocity by a factor depending on the values of reversal probabilities. However we can not relate the asymptotic equation (4.43) directly with the FPE. In order to do that we use of this asymptotic relation and write

$$
\frac{\partial^{2} P(x, t)}{\partial t^{2}}=v_{e f f}^{2} \frac{\partial^{2} P(x, t)}{\partial x^{2}} .
$$

Substituting back into equation (4.38), we obtain in the next approximation

$$
\begin{aligned}
\left(\lambda^{+}+\lambda^{-}\right) \frac{\partial P}{\partial t} & =v^{2} \frac{\partial^{2} P}{\partial x^{2}}+v\left(\lambda^{+}-\lambda^{-}\right) \frac{\partial P}{\partial x}-v_{e f f}^{2} \frac{\partial^{2} P}{\partial x^{2}} \\
\Rightarrow \frac{\partial P}{\partial t} & =-v\left(\frac{\lambda^{-}-\lambda^{+}}{\lambda^{-}+\lambda^{+}}\right) \frac{\partial P}{\partial x}+v^{2}\left[\frac{4 \lambda^{+} \lambda^{-}}{\left(\lambda^{+}+\lambda^{-}\right)^{3}}\right] \frac{\partial^{2} P}{\partial x^{2}} .
\end{aligned}
$$


which looks similar in the form as in the FPE for biased diffusion. But with an effective velocity as in equation (4.45) and an effective diffusion constant given by

$$
D_{e f f}=v^{2}\left[\frac{8 \lambda^{+} \lambda^{-}}{\left(\lambda^{+}+\lambda_{-}\right)^{3}}\right] \text {. }
$$

Using these relations for the effective velocity and the effective diffusion constant, we can calculate an effective length scale associated with the BPRW model. It reads,

$$
\lambda_{e f f}=\frac{D_{e f f}}{2 v_{e f f}}=4 v \frac{\lambda^{+} \lambda^{-}}{\left(\lambda^{+}+\lambda^{-}\right)^{2}\left(\lambda^{-}-\lambda^{+}\right)}=4 \Delta x \frac{q^{+} q^{-}}{\left(q^{+}+q^{-}\right)^{2}\left(q^{-}-q^{+}\right)},
$$

where, $\Delta x$ is the length of a single step in the BPRW model. If we assume that this value is exactly equal to the Peclet length $\lambda_{\mathrm{ch}}=D /\left(2 v_{\mathrm{ch}}\right)$ from the FPE approach then, the length scale $\Delta x$ which can be written as

$$
\Delta x=\frac{\left(q^{+}+q^{-}\right)^{2}\left(q^{-}-q^{+}\right)}{4 q^{+} q^{-}} \lambda_{\mathrm{ch}}=\frac{\left(q^{+}+q^{-}\right)^{2}\left(q^{-}-q^{+}\right)}{8 q^{+} q^{-}} \frac{D}{v_{\mathrm{ch}}} .
$$

should remain a constant (since the velocity, see figure 4.9 (a), of the persistent random walk as given in equation (4.45) is a constant ). Remember the constancy of this value will hold true if the BPRW model has a constant microscopic velocity given by $v=\Delta x / \Delta t$. In figure $4.9 \mathrm{~b}$ b) we have plotted the value of $\Delta x$ from equation (4.56) as a function of the length of the system. It shows that for shorter system sizes $\Delta x$ grows (to a good approximation exponentially). But once the system length exceeds approximately twice the Peclet length of the associated FPE, $2 \lambda_{\text {ch }}$, the value of $\Delta x$ remains nearly a constant. It is no surprise at all. In the framework of BPRW we have derived an effective FPE which is appropriate in the approximations of large spatial and temporal coordinates. Therefore, the crossover from BPRW to
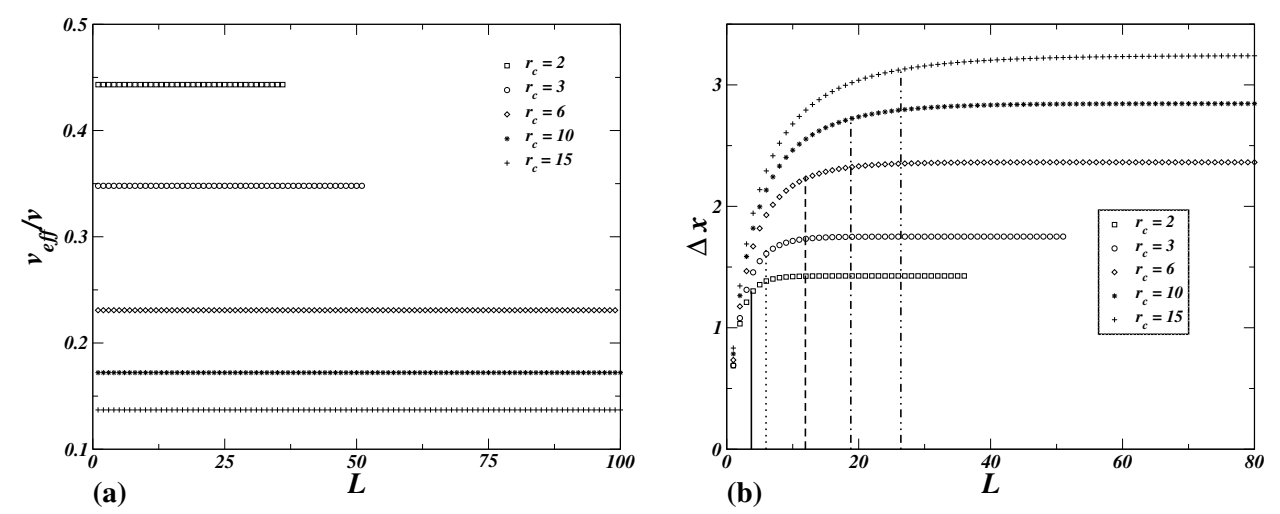

Figure 4.9: (a) The effective velocity in equation (4.45) as a function of the length of the waveguide remains a constant, whereas (b) the associated length scale $\Delta x$ given in equation (4.56) varies to a constant value. The vertical lines in (b) indicate the positions of twice the Peclet length $2 \lambda_{\mathrm{ch}}$. 
FPE will be expected to be of the order of the length scale where the momentum correlations vanish, which is of the order of $\lambda_{\mathrm{ch}}$. This explains the reason why the FPE approach was able to reproduce the asymptotic length scale associated with the system (even though it could not reproduce the pre-factor). Indeed, if we see the results of the FPE with the correct pre-factor, then we find that the results very well match with the numerical results of transmission probabilities after a value of $L \approx 2 \lambda_{c h}$.

Now we have to implement this picture in our system in order to understand the transmission in a finite channel. For this reason we divide the total length of the system into $n$ equal parts each measuring length $L_{1}$, such that $L=n L_{1}$. Each section of length $L_{1}$ can be treated as a scatterer which scatter particles to right hand side (transmission $t$ if the particle enters from left) or to left hand side (transmission $t^{\prime}<t$ if the particle enters from right hand direction). For our purpose the chaotic trajectories transmit in different ways for left to right than from right to left transmission, therefore $t \neq t^{\prime}$. Then the incoming probabilities can be related to the outgoing probabilities with the help of a classical scattering matrix. We can modify this picture to relate the probabilities on the right hand side with the probabilities of the left hand side of the scatterer. The matrix relating these probabilities is called the transfer matrix. The details of this picture and the relevant connections with the transmission in our model are the subjects of discussion in the following section of this chapter.

\subsection{Transfer Matrix Formalism for Transmission}

The classical scattering matrix relates the outgoing probabilities $\left(a_{+}, b_{-}\right)$in terms of the incoming probabilities $\left(a_{-}, b_{+}\right)$as

$$
\left(\begin{array}{l}
a_{+} \\
b_{-}
\end{array}\right)=S\left(\begin{array}{l}
a_{-} \\
b_{+}
\end{array}\right)=\left(\begin{array}{ll}
r & t^{\prime} \\
t & r^{\prime}
\end{array}\right)\left(\begin{array}{l}
a_{-} \\
b_{+}
\end{array}\right)
$$

where $S$ is the relevant scattering matrix for the scatterer (as shown in figure 4.8 with the incoming and outgoing probabilities given by $\left(a_{-}, b_{+}\right)=(\alpha(x-\delta, t), \beta(x-\delta, t))$ and $\left(a_{+}, b_{-}\right)=(\alpha(x, t+\tau), \beta(x-2 \delta, t+$ $\tau)$ ), respectively; and the transmission and the reflection probabilities are given by $t=p^{+}$and $t^{\prime}=p^{-}$. Accordingly $r=q^{+}$and $r^{\prime}=q^{-}$). In order to implement the transfer matrix model to our picture we must relate the probabilities at length $k L_{1}$ to the probabilities at length $(k-1) L_{1}$ i.e., we should relate the probabilities $\left(b_{-}, b_{+}\right)$with $\left(a_{-}, a_{+}\right)$. They are related by a transfer matrix $T$. From the relations of equation (4.57), we can find the relevant transfer matrix

$$
\left(\begin{array}{c}
b_{-} \\
b_{+}
\end{array}\right)=T\left(\begin{array}{l}
a_{-} \\
a_{+}
\end{array}\right)=\left(\begin{array}{cc}
\frac{t+t^{\prime}-1}{t^{\prime}} & \frac{1-t^{\prime}}{t^{\prime}} \\
\frac{-(1-t)}{t^{\prime}} & \frac{1}{t^{\prime}}
\end{array}\right)\left(\begin{array}{c}
a_{-} \\
a_{+}
\end{array}\right) .
$$

See Appendix Ffor details of relating the components of the scattering matrix with the components of the transfer matrix. Since we have $n$ such scatterers over the entire length of the channel, we will have $n$ 
(a)
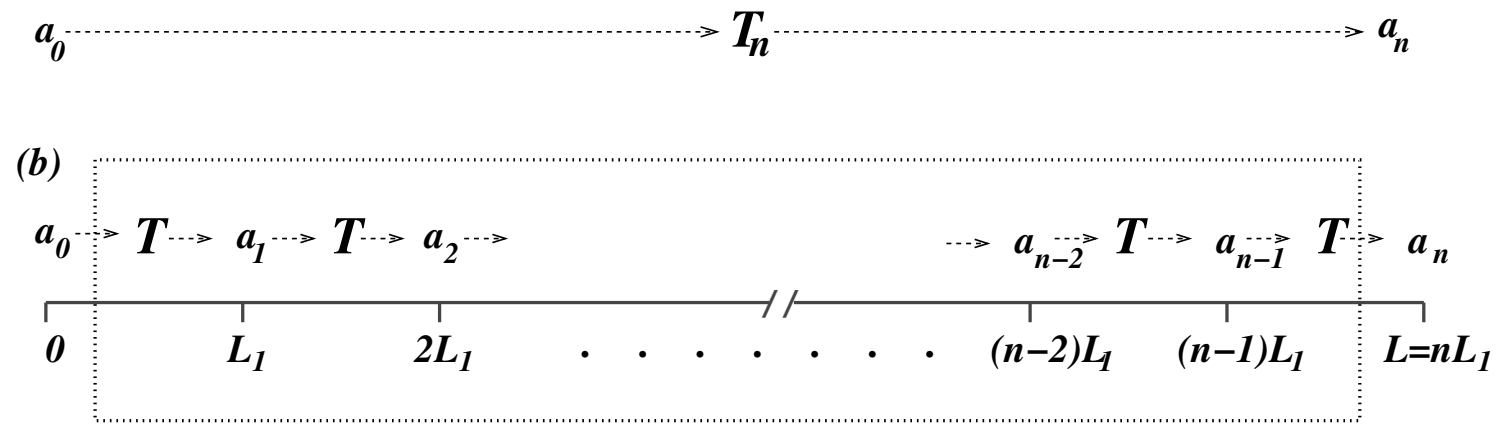

Figure 4.10: In the framework of transfer matrix the total transfer matrix $T_{n}$ in $(a)$ can be broken up into $n$ equivalent transfer matrices $T$ as in $(b)$. Then the final probability $a_{n}=T_{n} a_{0}$ is related to $a_{0}$ with $n$ number of $T$ matrices as in equation (4.62).

such matrices (as shown in figure 4.10 $(b)$ ) coupled to each other forming the transfer matrix $T_{n}$ for total transmission (as in figure 4.10 $(a)$ ). If $t_{n}$ is the total transmission probability in the forward direction (left to right transmission) and $t_{n}^{\prime}$ is the total transmission probability in the backward direction (right to left transmission), both being over the entire length of the channel, then we can write this transfer matrix in the same form as in equation (4.58) but with $t$ and $t^{\prime}$ replaced by $t_{n}$ and $t_{n}^{\prime}$, respectively. Therefore we obtain

$$
T_{n}=\left(\begin{array}{cc}
\frac{t_{n}+t_{n}^{\prime}-1}{t_{n}^{\prime}} & \frac{1-t_{n}^{\prime}}{t_{n}^{\prime}} \\
\frac{-\left(1-t_{n}\right)}{t_{n}^{\prime}} & \frac{1}{t_{n}^{\prime}}
\end{array}\right)=\left(\begin{array}{cc}
T_{n}^{1,1} & T_{n}^{1,2} \\
T_{n}^{2,1} & T_{n}^{2,2}
\end{array}\right)
$$

which relates $a_{n}$ to $a_{0}$ by

$$
a_{n}=T_{n} a_{0},
$$

where $a_{n}$ is the matrix containing the probabilities at the exit end of the channel and $a_{0}$ is the matrix containing probabilities at the entrance end of the channel. Since we have broken up the total length into $n$ equal parts each of length $L_{1}$ each part will have a transfer matrix given by equation (4.58). Therefore, if $a_{k}$ is the output amplitude for length $k L_{1}$ of the channel we can write

$$
a_{k}=T a_{k-1}=T T a_{k-2}=\ldots=\underbrace{T T \ldots T T}_{\mathrm{k}-\text { terms }} a_{0} .
$$

Hence, for the entire channel, which contain $n$ equal sections, we can write

$$
a_{n}=\underbrace{T T \ldots T T}_{\text {n-terms }} a_{0} .
$$


Comparing equations (4.60) and (4.62), we find

$$
T_{n}=\underbrace{T T \ldots T T}_{\mathrm{n} \text {-terms }} .
$$

Using this relation we can solve for the transmission probability $t_{n}$ when the particle enters from left, s.t., $a_{0}=(1,0)$ and also the transmission probability $t_{n}^{\prime}$ when the particle enters from right, s.t., $a_{0}=$ $(0,1)$. The details of this derivation are provided in Appendix $\mathrm{G}$. Then the solution will lead to the transmission probabilities as

$$
t_{n}=\frac{1-\frac{r^{\prime}}{r}}{\left(\frac{t^{\prime}}{t}\right)^{n}-\frac{r^{\prime}}{r}} \quad \text { and } \quad t_{n}^{\prime}=\frac{1-\frac{r}{r^{\prime}}}{\left(\frac{t}{t^{\prime}}\right)^{n}-\frac{r}{r^{\prime}}}
$$

Thus, for very large system length i.e., when $L \rightarrow \infty$ (or in terms of number of scatterers $n \rightarrow \infty$ ) we obtain

$$
t_{\infty}=\lim _{n \rightarrow \infty} t_{n}=\lim _{n \rightarrow \infty} \frac{1-r^{\prime} / r}{\left(t^{\prime} / t\right)^{n}-r^{\prime} / r}=1-\frac{r}{r^{\prime}} .
$$

and

$$
t_{\infty}^{\prime}=\lim _{n \rightarrow \infty} t_{n}^{\prime}=\lim _{n \rightarrow \infty} \frac{1-r / r^{\prime}}{\left(t / t^{\prime}\right)^{n}-r / r^{\prime}}=t_{\infty}\left(\frac{t}{t^{\prime}}\right)^{-n} .
$$

Moreover the expressions in equation (4.64) can be related with each other by

$$
t_{n}=\left(1-\frac{r}{r^{\prime}}\right)+\frac{r}{r^{\prime}} t_{n}^{\prime}
$$

which looks familiar in the form with the relation of the total transmission probability in one direction which has been provided in terms of the conditional probabilities of the chaotic and the regular trajectories of the opposite direction (refer to equation (4.8)). Indeed they will be exactly equal if we assume

$$
\mu_{\mathrm{reg}}=\left(1-\frac{r}{r^{\prime}}\right) \quad \text { and } \quad t_{n}^{\prime}=t_{\mathrm{ch}}^{\prime}(L) .
$$

But can we do so? As there is no direct connection between the parameters of BPRW and the dynamics of our original model, we must make use of the information about the underlying phase space structure in order to close this gap. The phase space dynamics suggests that as the system length $L$ increases to infinity the transmission saturates at a constant value determined only from the dynamics of the regular skipping trajectories (see equation (4.13). Therefore,

$$
t_{\infty}=1-\frac{r}{r^{\prime}}=\mu_{\mathrm{reg}} \quad \text { or } \quad \frac{r}{r^{\prime}}=\mu_{\mathrm{ch}} .
$$


We also can conclude this result from the relation between $t(L)$ and $t_{\mathrm{ch}}^{\prime}(L)$ which is given in equation (4.8) and reads

$$
t=\mu_{\mathrm{reg}}+\mu_{\mathrm{ch}} t^{\prime}
$$

In terms of the reversal probabilities $r$ and $r^{\prime}$, this equation changes to

$$
1-r=\mu_{\mathrm{reg}}+\mu_{\mathrm{ch}}\left(1-r^{\prime}\right)
$$

Solving this equation we will obtain the required result as in equation (4.69). Furthermore, we compare the results obtained within the FPE approach (see equation (4.21) ) and the results of transfer matrix (refer to equation (4.66)). This yields

$$
c \exp \left(-\frac{L}{\lambda_{\mathrm{ch}}}\right)=c \exp \left(-n \frac{L_{1}}{\lambda_{\mathrm{ch}}}\right)=\mu_{\mathrm{reg}}\left(\frac{t}{t^{\prime}}\right)^{-n} .
$$

Now it is easy to read off the correct pre-factor of the asymptotic exponential decay

$$
c=\mu_{\text {reg }} .
$$

On the other hand we can infer that

$$
\exp \left(\frac{L_{1}}{\lambda_{\mathrm{ch}}}\right)=\frac{t}{t^{\prime}}
$$

and substitute back these values into equations (4.64). The final result for the chaotic transmission probability from right to left is then

$$
t_{\mathrm{ch}}^{\prime}(L)=\frac{\mu_{\mathrm{reg}}}{\exp \left(L / \lambda_{\mathrm{ch}}\right)-\mu_{\mathrm{ch}}},
$$

while the total transmission is given by

$$
t(L)=\frac{\mu_{\mathrm{reg}}}{1-\mu_{\mathrm{ch}} \exp \left(-L / \lambda_{\mathrm{ch}}\right)} .
$$

The results of these equations (4.75) and (4.76) are compared to the numerical data in figure 4.11. From these figures, we can see small systematic errors for small values of cyclotron radius. These can be attributed to direct trajectories which escape from the system before being able to get scattered within it. Some examples are given in figure 4.12. The details of these trajectories will depend on the system parameters. In case of smaller cyclotron radius a trajectory entering at $x=0$ with a steep angle will again reach $x=0$ just by completing a simple arc (as in figure $4.12(a)$ ). From the figure we calculate

$$
r_{c} \sin \left(\frac{\pi}{2}+\varphi\right)=r_{c} \cos \varphi=\frac{y}{2} .
$$



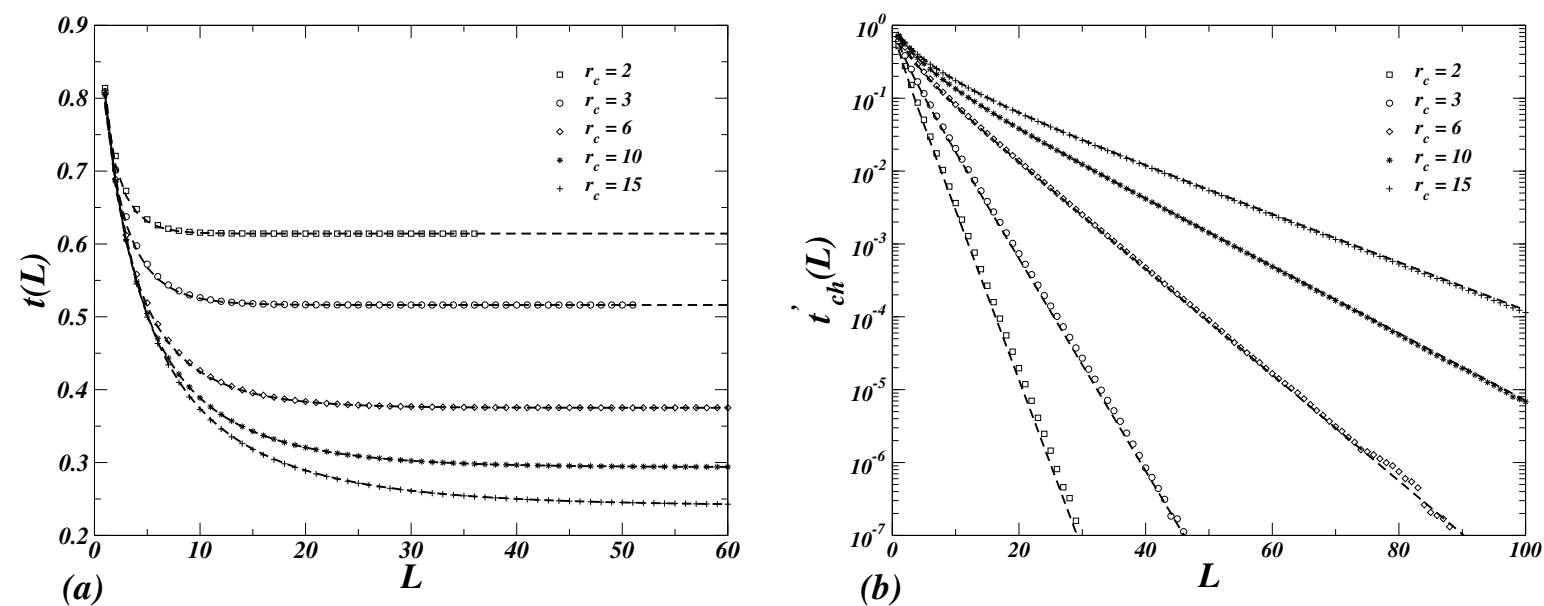

Figure 4.11: The final results for (a) total transmission (dashed line) from equation (4.76) is compared with the corresponding numerical results (in symbols) and $(b)$ the chaotic transmission (dashed line) from equation (4.75) is shown along with the numerical results (symbols). The predicted analytical results are in good agreement with the numerics even at small length scales.
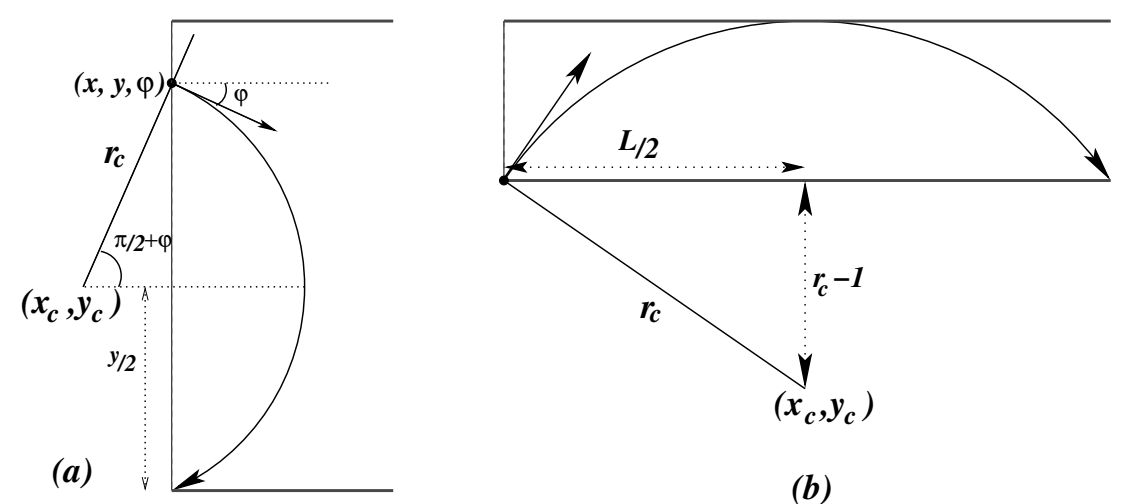

(b)

Figure 4.12: For $(a)$ small and $(b)$ large values of cyclotron radius some of the trajectories will escape the system without being scattered within the channel. These type of systematic errors will show small deviations in the expected values of transmission probabilities.

Therefore, if the incident angle $\varphi$ is such that

$$
r_{c} \cos \varphi \leqslant \frac{y}{2}
$$

then a trajectory will get reflected without being scattered within the system. Hence, the number of these trajectories will decrease as $r_{c}^{-1}$ and therefore they will lead to deviations from equation (4.76) for small cyclotron radius. Similarly for a large cyclotron radius a shallow incidence will result in direct 
transmission of trajectories (figure $4.12(b)$ ). From the geometry of the figure we can calculate that, a trajectory starting at $(x=0, y=0)$ will escape without scattering from the lower wall if

$$
\frac{L^{4}}{4}=r_{c}^{2}-\left(r_{c}-1\right)^{2}=2 r_{c}-1 \approx 2 r_{c},
$$

since the cyclotron radius is large. So, if the length of the system is such that

$$
L \leq \sqrt{8 r_{c}}
$$

then some of the trajectories will get transmitted through the channel before being scatterered within the system. Presence of these trajectories implies, there is a correlation among the incoming and outgoing trajectories. In our treatment this type of correlations is absent. Therefore, these trajectories can be more than just an extra phase space region where transmission or reflection is certain. They might lead to corrections in the transmission and reflection probabilities for the "main" chaotic components and this in return can result in an exponent differing from $\lambda_{\mathrm{ch}}$. Nonetheless these corrections are small and thus can be neglected as a first approximation.

However the FPE approach and the transfer matrix formulation within the BPRW model are mutually incompatible for short system sizes i.e., the length scale associated with the BPRW which can be calculated using equation (4.66)

$$
\tilde{\lambda}=\frac{L_{1}}{\ln \left(t / t^{\prime}\right)}
$$

is not compatible with the Peclet length associated with the FPE, $\lambda_{\mathrm{ch}}=D /\left(2 v_{\mathrm{ch}}\right)$. See the figure 4.13 for a comparison. But as the length of the system increases they come closer to each other. As

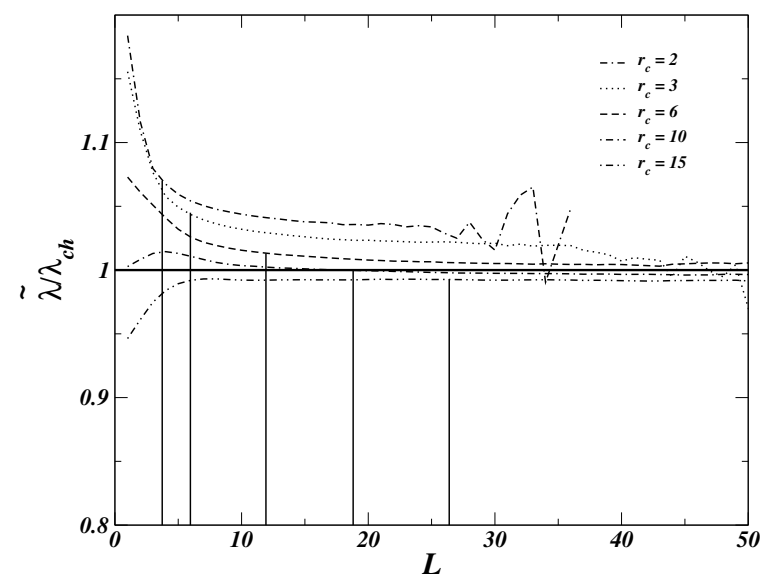

Figure 4.13: Ratio of effective Peclet length $\tilde{\lambda}$ within the BPRW approach, predicted from equation (4.81), to the Peclet length of FPE approach $\lambda_{\mathrm{ch}}$. The ratio remains close to unity. The vertical lines show the position of $2 \lambda_{\mathrm{ch}}$ for a comparison with the figure 4.9 
a comparison to the previous result in $\Delta x$ which shows a crossover around $2 \lambda_{\mathrm{ch}}$, the results obtained from the ratios of Peclet lengths is not encouraging. The possible explanation is that the equation (4.66) is valid only at the asymptotic scale, i.e., when $n \rightarrow \infty$, or the system lengths is very large. Therefore, the results will not be valid at shorter system lengths. In this regime of shorter system lengths the detailed analysis will involve the calculation of $r$ and $r^{\prime}$ based on the microscopic dynamics of the system (which are not immediately accessible based on system parameters).

There is one more important consequence of the transmission probabilities as given in equations (4.75) and (4.76). In deriving those results we have used an appropriate synthesis between the FPE and the BPRW. This reproduces the appropriate results for the transmission. This is not at all obvious. Indeed, in an analogous approach (see section 4.5) for the unbiased case the FPE approach is not able to provide a correct pre-factor for the Ohm's Law, while PRW yields

$$
t(L)=\frac{D}{v_{\mathrm{ch}} L} .
$$

\subsection{Ohm's Law: A FPE and PRW approach}

FPE approach provides the exit probabilities as given in equations (4.14) and (4.15). Assuming $v_{\mathrm{ch}} \rightarrow 0$ or $\lambda_{\mathrm{ch}} \rightarrow \infty$, we can approximate these equations to

$$
\pi_{L}^{\prime}(x)=\frac{\left(1-x / \lambda_{\mathrm{ch}}\right)-\left(1-L / \lambda_{\mathrm{ch}}\right)}{1-\left(1-L / \lambda_{\mathrm{ch}}\right)}=1-\frac{x}{L}
$$

and

$$
\pi_{L}(x)=\frac{1-\left(1-x / \lambda_{\mathrm{ch}}\right)}{1-\left(1-L / \lambda_{\mathrm{ch}}\right)}=\frac{x}{L} .
$$

Now the recursion relation (4.17) for transmission $t_{\mathrm{ch}}^{\prime}$, which reads

$$
t_{\mathrm{ch}}^{\prime}(L)=t_{\mathrm{ch}}^{\prime}(l) \pi_{L}^{\prime}(L-l)
$$

should still remain valid. It will provide,

$$
\begin{aligned}
t_{\mathrm{ch}}^{\prime}(L) & =t_{\mathrm{ch}}^{\prime}(l)\left(1-\frac{L-l}{L}\right) \\
\Rightarrow L t_{c h}^{\prime}(L) & =l t_{c h}^{\prime}(l) \\
\Rightarrow t_{\mathrm{ch}}^{\prime}(L) & \propto \frac{1}{L}
\end{aligned}
$$

This is Ohm's law with an unknown pre-factor. Whereas, within the BPRW approach we find, the equations for $t_{n}^{\prime}$ and $t_{n}$ as given in equation (4.64) are of the form $0 / 0$. Therefore, we need to look for 
the transfer matrix

$$
T=\left(\begin{array}{cc}
2-1 / t & 1 / t-1 \\
1-1 / t & 1 / t
\end{array}\right),
$$

and then determine the transmission for $n$ number of scatterers. We can calculate that

$$
T_{n}=\underbrace{T T \ldots T T}_{\mathrm{n} \text {-times }}=\left(\begin{array}{cc}
(n+1)-n / t & n / t-n \\
n-n / t & n / t-(n-1)
\end{array}\right)=\left(\begin{array}{cc}
2-1 / t_{n} & 1 / t_{n}-1 \\
1-1 / t_{n} & 1 / t_{n}
\end{array}\right) .
$$

This yields,

$$
\frac{1}{t_{n}}=\frac{n}{t}-(n-1)=\frac{1-t}{t} n+1
$$

Which can be further simplified, for large $n \rightarrow \infty$, to provide

$$
t_{n} \approx \frac{t}{1-t} \frac{1}{n}
$$

It remains to calculate an appropriate diffusion constant for the PRW. For this reason we need to take a look into the scattering map and then calculate an appropriate diffusion constant. See Appendix for details of this calculation. We find that the diffusion constant for the PRW satisfies the following equation

$$
D=\frac{t}{1-t} \frac{\Delta^{2} x}{\Delta t}
$$

Therefore, we get

$$
t(L)=t_{n}=\frac{D}{n} \frac{\Delta t}{\Delta^{2} x}=\frac{D}{v_{\mathrm{ch}}} \frac{1}{L}
$$

where

$$
v_{\mathrm{ch}}=\frac{\Delta x}{\Delta t} .
$$

This result is in agreement with the relation (4.82).

\subsection{Recursive Formulation for Transmission}

It was fascinating to observe that a novel combination of FPE and BPRW is able to reproduce the results of transmission on a wider scale. Independent of these we can use a different approach involving the addition of all the paths of a particle leading to transmission in order to find the transmission probabilities. This mechanism is described below.

Implementing the scattering picture (figure 4.14), we can calculate that a particle entering from left will get transmitted to right with probability $t$ and get reflected with probability $r=1-t$. Similarly for a particle entering from the other direction (right) will get transmitted with probability $t^{\prime}$ and get 


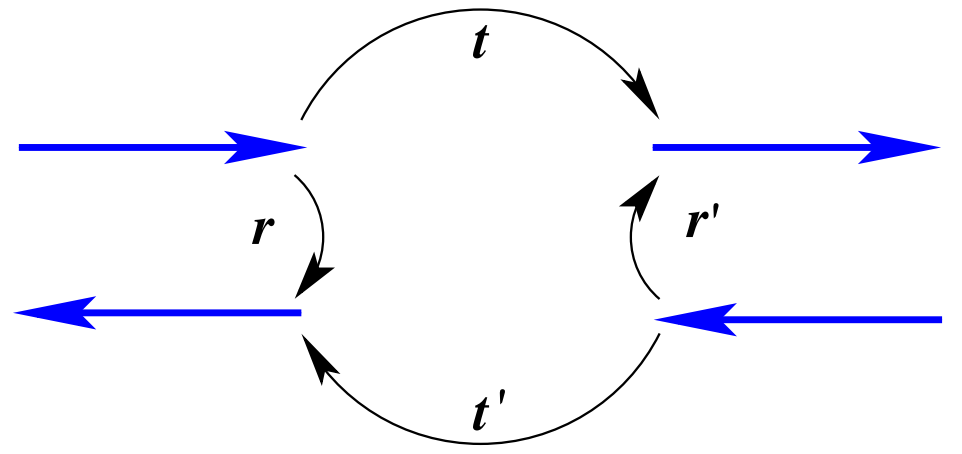

Figure 4.14: Scattering at a single impurity center. The particle entering from left will travel right with a transmission probability $t$, and will be reflected in the same direction with probability $r=1-t$. Similarly, the particle entering from right will be transmitted with probability $t^{\prime}$ and will be reflected with probability $r^{\prime}=1-t^{\prime}$.

reflected with probability $r^{\prime}=1-t^{\prime}$. This provides the transmission and reflection probabilities at a single impurity site (of length $L_{1}=L / n$ ) to be

$$
\begin{array}{ll}
t_{1}=t, & r_{1}=1-t_{1} \\
t_{1}^{\prime}=t^{\prime}, & r_{1}^{\prime}=1-t_{1}^{\prime} .
\end{array}
$$

where, $t_{1}$ and $r_{1}$ are the transmission and reflection probabilities of a particle entering from left for a single impurity center and the primes denote the equivalent ones if it enters from right.

However, if we add another scattering site to the existing one (see figure 4.15), the particle might have to undergo multiple reflections between the two scatterers before coming out in any one of the directions. For simplicity we elaborate the transmission from left to right. A particle entering from

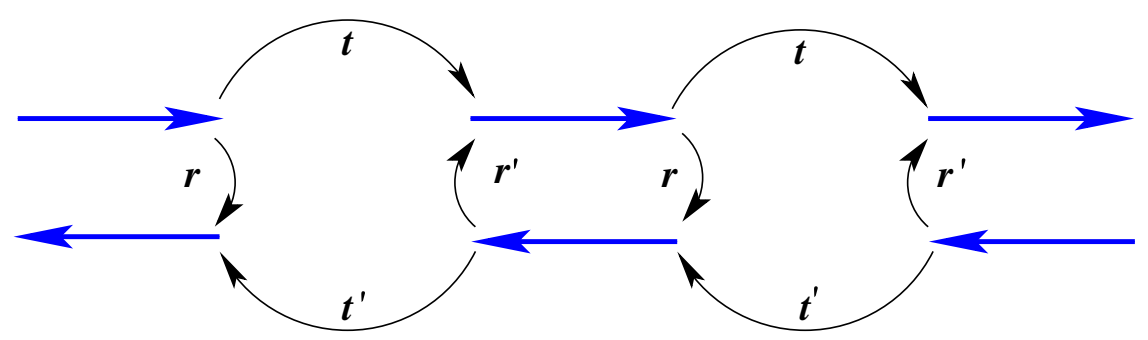

Figure 4.15: Scattering at two impurity centers. The particle entering from left/right might have to perform multiple maneuvers (multiple reflections) between the two centers before being transmitted/reflected in any of the directions. The total probability of transmission/reflection will be then the addition of all the possible paths leading to the final destination. 


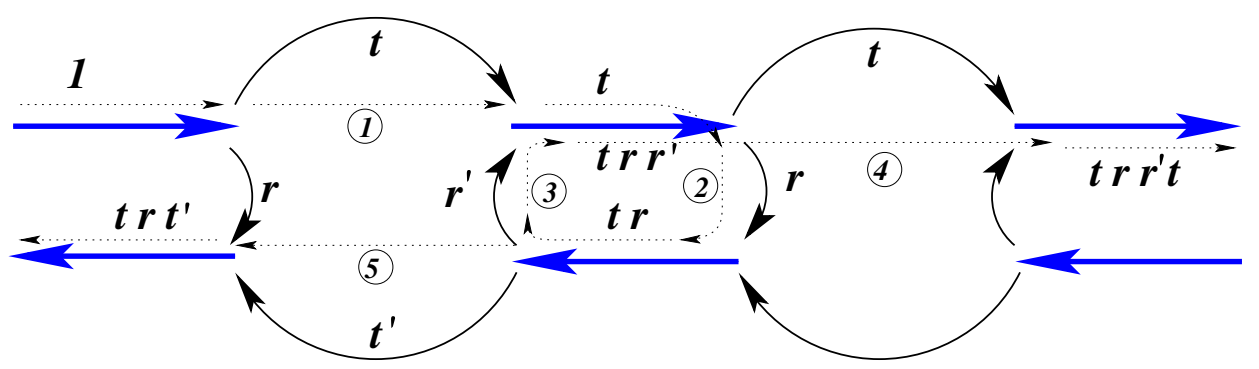

Figure 4.16: For a one loop transmission (path 1 (transmission $t) \rightarrow 2$ (reflection $r$ ) $\rightarrow 3$ (reflection $r^{\prime}$ ) $\rightarrow 4$ (transmission $t$ ), denoted inside circles), the total contribution will be the multiplication of individual probability contributions at each point of transmission/reflection. This provides a total contribution of $t r r^{\prime} t$ to one loop transmission. Then the total probability to get transmitted across the two sites will be the addition of many such loops. Similarly if the particle get reflected (path 1 (transmission $t) \rightarrow 2$ $($ reflection $r) \rightarrow 5$ (transmission $\left.t^{\prime}\right)$ ) the contribution to reflection will be $t r t^{\prime}$ and the total probability of reflection for two sites will be addition of several of these type of paths. (see text for details)

the left might pass across the two sites directly. Then the probability of transmission will be $t$ for the first site and then $t$ also at the second site, providing the transmission probability to be $t^{2}$. However, if instead of directly transmitting across the second site it performs a single loop reflection between them (figure 4.16) and then finally comes out in the right direction, the transmission probability will be $t$ for the transmission at the first site, $r$ for the reflection at the second site, $r^{\prime}$ for another reflection at the first site, and finally $t$ at the last site. This provides the the transmission probability for a single loop to be $t^{2} r r^{\prime}$. Similarly we can have $4,6,8, \ldots$ number of reflections ( $n$ numbers each from both the sites for $2 n$ number of reflections) before being transmitted through two sites. This will provide the total transmission probability (for left to right transmission) for two sites to be

$$
\begin{aligned}
t_{2} & =t^{2}+t\left(r r^{\prime}\right) t+t\left(r r^{\prime}\right)^{2} t+t\left(r r^{\prime}\right)^{3} t+\ldots \\
& =t^{2} \sum_{n=0}^{\infty}\left(r r^{\prime}\right)^{n} \\
& =\frac{t^{2}}{1-r r^{\prime}} \\
& =\frac{t t_{1}}{1-r r_{1}^{\prime}}
\end{aligned}
$$

The reason for the last modification will be clear once we do the calculations for three or more impurity sites. But before going to calculate the contributions for three or more sites, we need to find out the contribution of reflection in the left direction. In a similar way, as in the transmission for two sites, we 
can calculate the reflection probability by summing over all the possible paths leading to reflection

$$
\begin{aligned}
r_{2} & =r+t r t^{\prime}+t r\left(r^{\prime} r\right) t+\operatorname{tr}\left(r^{\prime} r\right)^{2} t+\ldots \\
& =r+r t t^{\prime} \sum_{n=0}^{\infty}\left(r^{\prime} r\right)^{n}=r\left(1+\frac{t t^{\prime}}{1-r^{\prime} r}\right)=r_{1}+\frac{t_{1} r t_{1}^{\prime}}{1-r r_{1}^{\prime}} .
\end{aligned}
$$

Note that

$$
\begin{aligned}
r_{2}+t_{2} & =r_{1}+\frac{t_{1} r t_{1}^{\prime}}{1-r r_{1}^{\prime}}+\frac{t t_{1}}{1-r r_{1}^{\prime}} \\
& =\frac{r_{1}\left(1-r r_{1}^{\prime}\right)+t_{1} r t_{1}^{\prime}+t t_{1}}{1-r r_{1}^{\prime}} \\
& =\frac{r_{1}\left(1-r r_{1}^{\prime}\right)+t_{1}\left(r t_{1}^{\prime}+t\right)}{1-r r_{1}^{\prime}} \\
& =\frac{r_{1}\left(1-r r_{1}^{\prime}\right)+t_{1}\left[r\left(1-r_{1}^{\prime}\right)+(1-r)\right]}{1-r r_{1}^{\prime}} \\
& =1,
\end{aligned}
$$

in conformity with the conservation of probability. Similar procedure for the transmission in the opposite direction (left to right) will lead to the transmission probability

$$
\begin{aligned}
t_{2}^{\prime} & =t^{\prime 2}+t^{\prime}\left(r^{\prime} r\right) t^{\prime}+t\left(r^{\prime} r\right)^{2} t^{\prime}+t^{\prime}\left(r^{\prime} r\right)^{3} t^{\prime}+\ldots \\
& =\frac{t^{\prime 2}}{1-r^{\prime} r}=\frac{t^{\prime} t_{1}^{\prime}}{1-r r_{1}^{\prime}},
\end{aligned}
$$

and the reflection probability

$$
\begin{aligned}
r_{2}^{\prime} & =r^{\prime}+t^{\prime} r^{\prime} t+t^{\prime} r^{\prime}\left(r r^{\prime}\right) t+t^{\prime} r^{\prime}\left(r r^{\prime}\right)^{2} t+\ldots \\
& =r^{\prime}\left(1+\frac{t^{\prime} t}{1-r r^{\prime}}\right) \\
& =r^{\prime}+\frac{t^{\prime} r_{1}^{\prime} t}{1-r r_{1}^{\prime}} .
\end{aligned}
$$

When we add a third site, to the existing two (see figure 4.17 (a)), the entire calculation becomes a mess. To simplify, we replace the first two sites with a single scatterer with the reflection and transmission probabilities as in equations (4.95)-(4.99). The net will be a wire involving two scatterers (as 


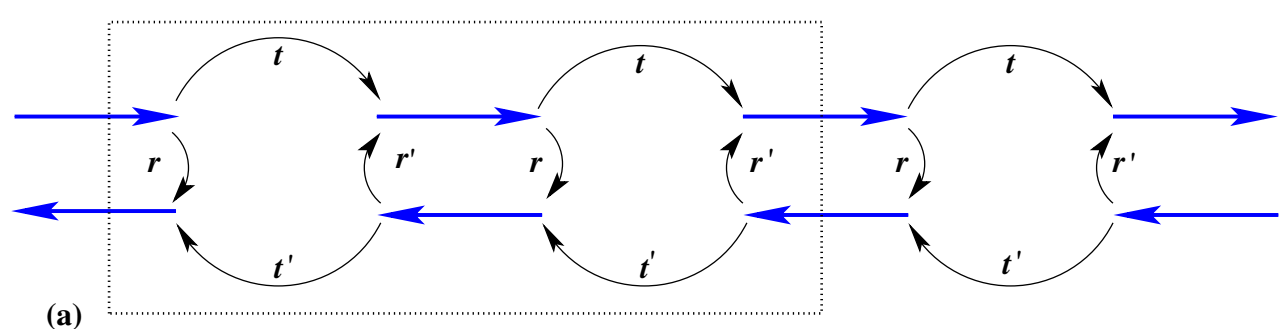

(a)

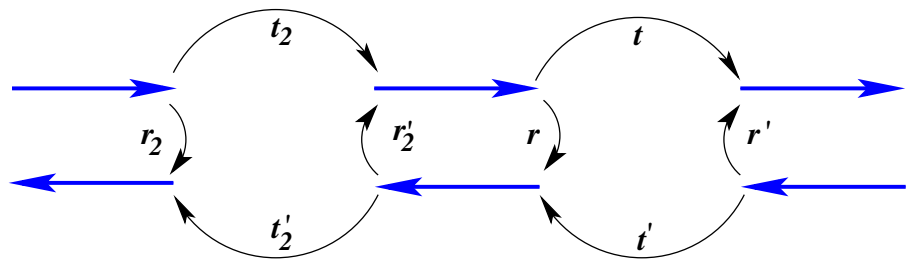

(b)

Figure 4.17: (a) Scattering at three impurity centers. The particle entering from left/right will have to go through trajectories with complex patterns involving many orders of reflections between each sites with various degrees. Therefore, for simplification purpose, we replace the first two sites with an effective scatterer with appropriate reflection/transmission probabilities as shown in (b).

shown in figure 4.17(b)), one with an effective transmission and reflection probabilities of $t_{2}, r_{2}$ (for left to right transmission and reflection), and $t_{2}^{\prime}$ and $r_{2}^{\prime}$ (for right to left transmission and reflection); and another with transmission and reflection probabilities of $t, r$ for one direction (left to right transmission), and $t^{\prime}, r^{\prime}$ for the other direction (right to left transmission) of transport. Now, we can repeat the calculations of two scattering sites to calculate the transmission and reflection probabilities for three sites,

$$
\begin{aligned}
t_{3} & =t_{2} t+t_{2}\left(r r_{2}^{\prime}\right) t+t_{2}\left(r r_{2}^{\prime}\right)^{2} t+\ldots \\
& =t_{2} t \sum_{n=0}^{\infty}\left(r r_{2}^{\prime}\right)^{n}=\frac{t t_{2}}{1-r r_{2}^{\prime}}
\end{aligned}
$$

for the left to right transmission and

$$
\begin{aligned}
r_{3} & =r_{2}+t_{2} r t_{2}^{\prime}+t_{2} r\left(r_{2}^{\prime} r\right) t_{2}^{\prime}+t_{2} r\left(r_{2}^{\prime} r\right)^{2} t_{2}^{\prime}+\ldots \\
& =r_{2}+r t_{2} t_{2}^{\prime} \sum_{n=0}^{\infty}\left(r_{2}^{\prime} r\right)^{n} \\
& =r_{2}+\frac{t_{2} r t_{2}^{\prime}}{1-r r_{2}^{\prime}} .
\end{aligned}
$$

for the left to left reflection. Similarly the transmission and reflection probabilities for a particle entering 
from the right hand side can be calculated using the same geometry as

$$
\begin{aligned}
t_{3}^{\prime} & =t^{\prime} t_{2}^{\prime}+t^{\prime}\left(r_{2}^{\prime} r\right) t_{2}^{\prime}+t^{\prime}\left(r_{2}^{\prime} r\right)^{2} t_{2}^{\prime}+\ldots \\
& =t^{\prime} t_{2}^{\prime} \sum_{n=0}^{\infty}\left(r_{2}^{\prime} r\right)^{n}=\frac{t^{\prime} t_{2}^{\prime}}{1-r r_{2}^{\prime}}
\end{aligned}
$$

and

$$
\begin{aligned}
r_{3}^{\prime} & =r^{\prime}+t^{\prime} r_{2}^{\prime} t+t^{\prime} r_{2}^{\prime}\left(r r_{2}^{\prime}\right) t+t^{\prime} r_{2}^{\prime}\left(r r_{2}^{\prime}\right)^{2} t+\ldots \\
& =r^{\prime}+t^{\prime} r_{2}^{\prime} t \sum_{n=0}^{\infty}\left(r r_{2}^{\prime}\right)^{n}=r^{\prime}+\frac{t^{\prime} r_{2}^{\prime} t}{1-r r_{2}^{\prime}}
\end{aligned}
$$

In general, we can continue this calculation with addition of more and more scattering sites and end up with a recursion relation between the transmission and reflection probabilities for $n+1$ sites in terms of the transmission and/or reflection probabilities for $n$ scattering centers, yielding

$$
\begin{array}{rlrl}
t_{n+1}=\frac{t t_{n}}{1-r r_{n}^{\prime}}, & r_{n+1} & =r_{n}+\frac{t_{n} r t_{n}^{\prime}}{1-r r_{n}^{\prime}} \\
t_{n+1}^{\prime} & =\frac{t^{\prime} t_{n}^{\prime}}{1-r r_{n}^{\prime}}, & r_{n+1}^{\prime} & =r^{\prime}+\frac{t^{\prime} r_{n}^{\prime} t}{1-r r_{n}^{\prime}}
\end{array}
$$

where, $t_{1}=t, r_{1}=r, t_{1}^{\prime}=t^{\prime}$ and $r_{1}^{\prime}=r^{\prime}$. To check the conservation of probabilities we observe that

$$
\begin{aligned}
t_{n+1}+r_{n+1} & =\frac{t t_{n}}{1-r r_{n}^{\prime}}+r_{n}+\frac{t_{n} r t_{n}^{\prime}}{1-r r_{n}^{\prime}}=\frac{t t_{n}+r_{n}\left(1-r r_{n}^{\prime}\right)+t_{n} r t_{n}^{\prime}}{1-r r_{n}^{\prime}} \\
& =\frac{r_{n}\left(1-r r_{n}^{\prime}\right)+t_{n}\left(t+r t_{n}^{\prime}\right)}{1-r r_{n}^{\prime}}=\frac{r_{n}\left(1-r r_{n}^{\prime}\right)+t_{n}\left[1-r+r\left(1-r_{n}^{\prime}\right)\right]}{1-r r_{n}^{\prime}} \\
& =r_{n}+t_{n}=1,
\end{aligned}
$$

using the method of induction. Similarly $t_{n}^{\prime}+r_{n}^{\prime}=1$. Therefore, the scattering matrix for $n+1$ sites will be

$$
S_{n+1}=\left(\begin{array}{cc}
r_{n+1} & t_{n+1}^{\prime} \\
t_{n+1} & r_{n+1}^{\prime}
\end{array}\right)=\left(\begin{array}{cc}
r_{n}+\frac{t_{n} r t_{n}^{\prime}}{1-r r_{n}^{\prime}} & \frac{t^{\prime} t_{n}^{\prime}}{1-r r_{n}^{\prime}} \\
\frac{t t_{n}}{1-r r_{n}^{\prime}} & r^{\prime}+\frac{t^{\prime} r_{n}^{\prime} t}{1-r r_{n}^{\prime}}
\end{array}\right) .
$$

Now we need to know only the transmission/reflection probabilities for a single scatterer $t, t^{\prime}$ (or $\left.r, r^{\prime}\right)$ in order to calculate the transmission/reflection probabilities for any length of the wire. Since $t, t^{\prime}$ for a scatterer of length $L_{1}$ depend on the microscopic details of our system we obtain them from numerics and then use the recursion relations to find the transmission/reflection probabilities for the 


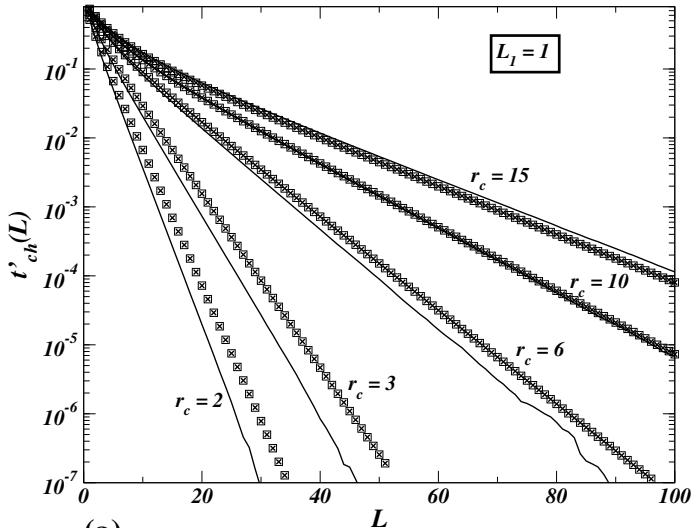

(a)

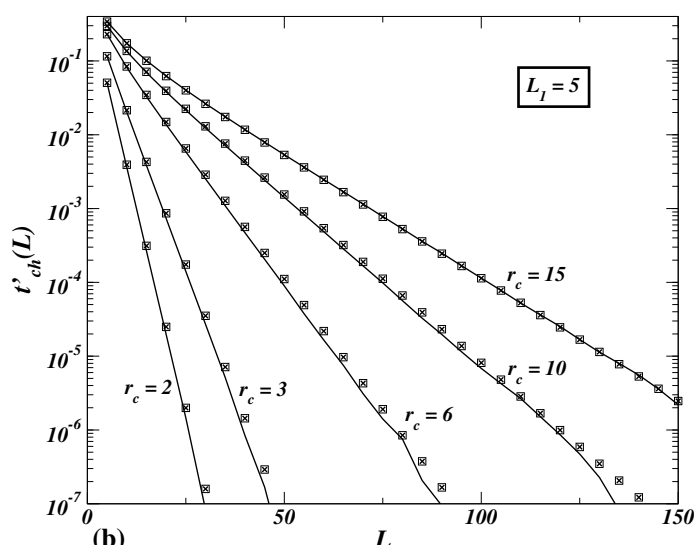

(b)

Figure 4.18: The results of the recursive formulation for the chaotic transmission $t_{n}^{\prime}(\times)$ are compared with the actual numerical data (lines) and the results of the transfer matrix formalism (in squares, from equations (4.64) for scatterer segments of length (a) $L_{1}=1$ and (b) $L_{1}=5$. We observe good agreement between the results of Recursive formalism and the transfer matrix formalism, but they do not agree with the actual numerical results for small $L_{1}$. However the results nearly match each other with the numerical results for the value of $L_{1}=5$. This shows that there is a length scale associated with the scattering matrix formalism or the recursive formalism.

other units. The results of theses calculations are shown in figure 4.18 (a) for the length of segment $L_{1}=1$ and in figure 4.18 (b) for $L_{1}=5$. We find that the results of this analysis agrees with the calculations of transfer matrix (equation (4.64)). Moreover, for small cyclotron radius and for small

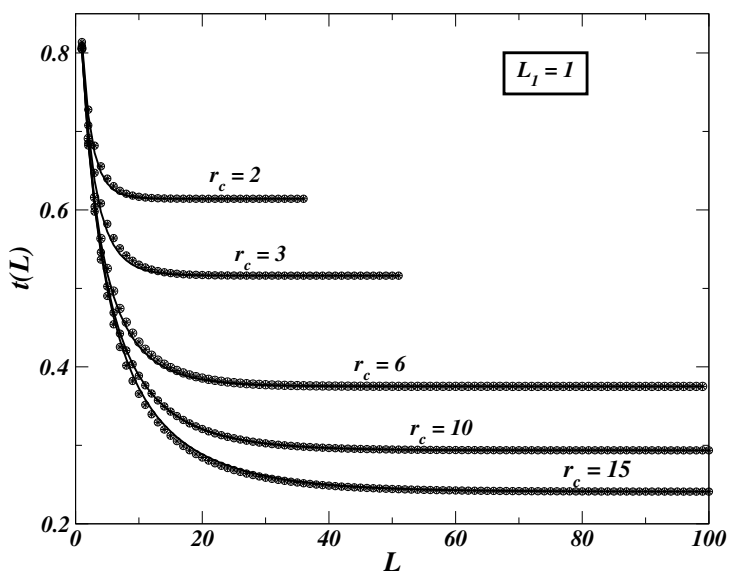

Figure 4.19: Same as in figure 4.18, but only for $t_{n}$ when the length scale of the scatterer $L_{1}=1$. It shows the matching of transmission results with each other $(*$ :recursive, circle:transfer matrix and line:numerical data). The reason for this matching is that the asymptotic decay of $t_{n}$ to $\mu_{\text {reg }}$ completely takes over the discrepancies observed with the results of $t_{n}^{\prime}$ at $L_{1}=1$ (see figure 4.18 (a)). 
value of segment dimension $\left(L_{1}=1\right.$ in figure $\left.4.18(\mathrm{a})\right)$ the results stay above the actual numerical values whereas for larger cyclotron radius they stay below the numerical results. However as we increase the value of $L_{1}$, the results come closer to the numerical values (see figure 4.18 (b) where the segment length was chosen to be $L_{1}=5$ ). In comparison to the length scale associated with the results of $t_{n}^{\prime}$, we find the results for $t_{n}$ match each other even at $L_{1}=1$. The simple reason for this disagreement is that the errors between the numerical and the transfer matrix/recursive formalism is very small compared to the value of $\mu_{\text {reg. }}$. Thus, the asymptotic results agree very much even at the smaller length scale. Nevertheless there are small differences between the actual numerical results and the results of transfer matrix/recursive formulation (even at $L_{1}=5$ ). These can be attributed to the trajectories escaping the system (either via reflection or direct transmission) without getting scattered from the lower wall (diffusive wall) of the channel.

We have seen in the microscopic model of BPRW that the occupational probability satisfy an effective FPE at higher values of time and length. Also we have seen that, when the models (FPE and BPRW) are combined together, they are able to reproduce the correct length scale associated with the decay of the transmission probability and also the correct pre-factor associated with that. Can we reproduce some of these results based on the recursive formalism? Indeed we can. If we look into the scattering matrix for $n$-sites (see equation (4.106)), then at asymptotic length scales of the system i.e., when $L \rightarrow \infty$ or equivalently when $n \rightarrow \infty$, then $t_{n}^{\prime}$ decays exponentially $t_{n} \rightarrow t_{\infty}$ (a constant value). Accordingly, $r_{n}^{\prime}$ approaches unity and $r_{n} \rightarrow 1-t_{\infty}$. Substitution of $r_{n}^{\prime}=1$ in the term $t_{n+1}^{\prime}$ of the scattering matrix, equation (4.106), will produce

$$
t_{n+1}^{\prime}=\left.\frac{t^{\prime} t_{n}^{\prime}}{1-r r_{n}^{\prime}}\right|_{r_{n}^{\prime}=1}=\frac{t^{\prime}}{1-r} t_{n}^{\prime}=\frac{t^{\prime}}{t} t_{n}^{\prime},
$$

which has a solution

$$
t_{n}^{\prime} \sim\left(\frac{t^{\prime}}{t}\right)^{n}
$$

Therefore, the pre-factor still remain undetermined. Nevertheless, the transfer matrix approach produces the correct pre-factor. If we look into the equations (4.66) and take the limit $n \rightarrow \infty$, then for $t>t^{\prime}$ we get

$$
\lim _{n \rightarrow \infty} t_{\infty}^{\prime}=\left(1-\frac{r}{r^{\prime}}\right)\left(\frac{t^{\prime}}{t}\right)^{n} .
$$

Therefore, what remains is to prove that $\left(t^{\prime} / t\right)^{n}$ decays exponentially with an appropriate length scale and find out this length scale within the framework of BPRW. The continuous version of BPRW leading to an equivalent FPE suggests that the spatial and the temporal coordinates need to be very large which has been taken care of by assuming $n \rightarrow \infty$. By writing $\left(t^{\prime} / t\right)^{n}$ in the form

$$
\left(\frac{t^{\prime}}{t}\right)^{n}=\left(1-\frac{t-t^{\prime}}{t}\right)^{n}=\left(1-\frac{r^{\prime}-r}{1-r}\right)^{n} .
$$


we can observe that it decays exponentially for large $n$. To reproduce a length factor, we note from the effective velocity and the effective diffusion constant within the BPRW (from equations (4.45) and (4.54), that

$$
v_{e f f}=v \frac{r^{\prime}-r}{r+r^{\prime}} \quad \text { and } \quad D_{e f f}=v^{2} \tau \frac{8 r^{\prime} r}{\left(r+r^{\prime}\right)^{3}},
$$

where $\tau$ is the time scale associated with the velocity $v$ and they are related with each other by a length scale $\delta$

$$
v=\frac{\delta}{\tau}
$$

Now $D_{\text {eff }}$ can be written using $v_{e f f}$ as

$$
\begin{aligned}
D_{e f f} & =v^{2} \tau \frac{2}{r+r^{\prime}}\left[1-\frac{\left(r^{\prime}-r\right)^{2}}{\left(r+r^{\prime}\right)^{2}}\right] \\
& =v^{2} \tau \frac{2}{r+r^{\prime}}\left[1-\left(\frac{v_{e f f}}{v}\right)^{2}\right] .
\end{aligned}
$$

Looking at the figure 4.9 (a) we observe that $\left(\frac{v_{e f f}}{v}\right)^{2} \ll 1$. Therefore, we can safely assume,

$$
D_{e f f} \approx v^{2} \tau \frac{2}{r+r^{\prime}}
$$

Note that this assumption will be true as long as $v_{e f f}^{2} \ll v^{2}$ which means weak bias for the electrons inside the channel. Therefore, for weak bias of electrons we get

$$
v_{e f f}=v \frac{r^{\prime}-r}{r+r^{\prime}} \quad \text { and } \quad D_{e f f}=v^{2} \tau \frac{2}{r+r^{\prime}}
$$

Using these two equations and the approximation that $r \rightarrow 0, r^{\prime} \rightarrow 0$ (in the continuum limit) we can approximate

$$
\frac{r^{\prime}-r}{1-r} \approx \delta \frac{2 v_{e f f}}{D_{e f f}}=\frac{L}{n \lambda_{e f f}} .
$$

where,

$$
\lambda_{e f f}=\frac{D_{e f f}}{2 v_{e f f}} \quad \text { and } \quad \delta=L / n .
$$

Substituting back into equation (4.110) yields

$$
\begin{aligned}
\lim _{n \rightarrow \infty}\left(1-\frac{r^{\prime}-r}{1-r}\right)^{n} & =\lim _{n \rightarrow \infty}\left(1-\frac{L}{n \lambda_{e f f}}\right)^{n} \\
& =\exp \left(-\frac{L}{\lambda_{e f f}}\right) .
\end{aligned}
$$


Therefore, the chaotic transmission $t_{n}^{\prime}$ will decay in an exponential way

$$
t^{\prime}(L) \sim \exp \left(-\frac{L}{\lambda_{e f f}}\right) .
$$

On the other hand the transfer matrix approach will yield

$$
t^{\prime}(L)=\left(1-\frac{r}{r^{\prime}}\right) \exp \left(-\frac{L}{\lambda_{e f f}}\right)=c \exp \left(-\frac{L}{\lambda_{c h}}\right) .
$$

which has a solution of the form

$$
c=c_{1}\left(1-\frac{r}{r^{\prime}}\right) \quad \text { and } \quad \frac{1}{\lambda_{\mathrm{ch}}}=\frac{1}{\lambda_{e f f}}+\frac{\ln c_{1}}{L} .
$$

$c_{1}$ remains undetermined. Therefore, the results of the FPE are in general not compatible with the results of BPRW. But if $c_{1}=1$ then it will infer that

$$
1-\frac{r}{r^{\prime}}=c \quad \text { and } \quad \lambda_{\mathrm{ch}}=\lambda_{e f f} .
$$

This confirms that if the bias is small and the length of the system is very large then the chaotic transmission $t^{\prime}$ should decay exponentially with an length scale determined from the velocity and the diffusion constant of the system. It shows the connection between the length scales of the exponential decay within the FPE and the BPRW.

Again for the Ohm's law the transmission should decay as inverse of length

$$
t_{n}=t_{n}^{\prime}=\frac{\gamma}{n}
$$

where $\gamma$ is a constant. Substituting this value into the scattering matrix (4.106), yields

$$
t_{n+1}=\frac{\gamma}{n+1}=\frac{t(\gamma / n)}{1-(1-t)(1-\gamma / n)} .
$$

Solving for $\gamma$ we find it satisfies,

$$
\gamma=\frac{t}{1-t}
$$

in conformity with the results obtained using the transfer matrix formalism (see section 4.5) for details.

What we find from the recursive formalism is that the results of it are not different from the results of transfer matrix formulation. Also we find that the results of FPE and transfer matrix/recursive formulation are able to reproduce the asymptotic relation of the $t^{\prime}(L)$. Moreover for weak bias the Peclet lengths within FPE and BPRW can be equated to each other. 


\subsection{Summary and Discussion}

In this chapter we have extended the discussion of the rough billiard model, introduced in chapter three for transport, for transmission of carriers across a disordered wire. In short we discussed the effect of non-diffusive behavior of electrons on the conductance within a finite wire. The most intriguing feature of our model is its simple structure and the description based on a novel combination of few random walks. From structural point of view, the regular trajectories transport without any back scattering in a single direction. In addition there is a chaotic component in the same direction as the regular ones. Whereas in the other direction the entire dynamics of the electrons is chaotic and cannot be handled easily. Nevertheless because of equilibrium, the total current inside the wire must be zero. This made us to describe the transmission dynamics of chaotic electrons moving in one direction in terms of the components of regular and the chaotic ones moving in the opposite direction. As the regular trajectories are a part of an integrable component, it remained to understand how the chaotic trajectories are moving against the direction of their average velocity. To treat them on the basis of a random walk, we use the simple convection-diffusion equation (FPE), which is able to describe the dynamics and the transmission at the asymptotic regime, where the things are much simpler. But it is unable to reproduce the correct pre-factor and the understanding of small scale deviations. However the BPRW model is able to describe the dynamics only on the basis of the microscopic dynamics of the system. Again neither of them are able to describe the correct picture of the model individually. Nonetheless the combination of these two provided a good agreement with the results of numerics. It is not at all obvious. We have seen how the FPE is not able to provide any pre-factor in case of the Ohm's law whereas the BPRW is able to predict the correct pre-factor. Therefore, in approaching this problem only by a single model (FPE which do not take care of correlations and BPRW which do take care of correlations) will not solve the entire problem. For shorter length scales we need to take care of momentum correlations which are much more important for the conductance in a finite wire, whereas for larger length scales diffusion dominates which can be treated within the FPE.

Within the continuum approach of BPRW, we are able to show a regime where the momentum correlations does not play much role and we can treat the electrons as diffusive objects. This crossover from the correlated behavior to the diffusive behavior occur at a length scale which is of the order of $2 \lambda_{\mathrm{ch}}$. This is not at all obvious. The fact is, after the Peclet length scale the dynamics of the electrons essentially becomes diffusive and therefore the FPE can be applied safely with the correct pre-factor. Therefore, on treating the electrons as random objects can be useful in reproducing the results for larger length and larger time scales of the system. But for the shorter length scales there is a competition between the momentum correlations and the diffusive dynamics of the electrons. At these time scales the electron will not have accessed the required phase space region, so that they effectively can be treated as random objects. Therefore the FPE fails. In order to take care of such effects, we use the BPRW model. But the problem of the BPRW model is we need the access to the microscopic dynamics 
of the electrons which itself is a problem. Some of the properties of transmission can be accessed within the dynamics of the regular trajectories (providing the value of asymptotic conductance) and the FPE model (providing the correct exponential length scale). The result is a combined model describing the conductance properties of the electron inside a finite disordered wire at any length scales.

Moreover, within the Recursive/Transfer matrix formulation we show that the length sclaes of the FPE and the BPRW can be equal provided we have weak bias of the electrons inside the channel and the constant factor $c_{1}=1$ (refer to equation (4.121)). It is known that 1D systems with disorder has localization effects and universal conductance fluctuations decaying to zero as the length of the system $L \rightarrow \infty$. In contrast, our system showing directed chaos has a value of conductance which remains a constant as the wire length $L \rightarrow \infty$. These contradicting statements can be reconciled as follows. It is known that in such systems with directed chaos the localization length diverges with the number of transversal modes. Therefore, even for a moderate number of modes the localization length would exceed the sample length. In treating the electrons classically, the number of transversal modes $N>>1$. Therefore, in this regime, we can ignore the localization effects providing the required result. 


\section{Chapter 5}

\section{Delay Time Distribution}

And these little things may not seem like much but after a while they take you off on a direction where you may be a long way off from what other people have been thinking about.

Roger Penrose

\subsection{Introduction}

The problem of time delay in quantum scattering was first considered by Eisenbud (in his Ph.D. thesis) and E. P. Wigner [94]. Later Felix T. Smith [95] extended the discussions to multi channel modes providing a lifetime matrix. This matrix provides a way to calculate the Wigner-Smith time delay which is interpreted as the typical time spent by the particle (undergoing scattering) in the interaction region. With the growing interest in studies of disordered mesoscopic systems, the Wigner time delay has attracted a great deal of attention in the domain of chaotic scattering. So, the properties of time delay have been studied in disordered systems and dispersive media [96, 97, 98, 99, 100, 101] even in cases of localized waves [102, 103, 104, 105]. Because of the original problem (of phase delay) in scattering systems, analysis of time delay has been used to understand the properties of chaotic scattering using microwave experiments $[106,107,108,109,110]$; and to understand the behavior of absorption [111, 112]. More recently time delay properties has been implemented as a way to probe the eigenfunction fractality in power-law banded random matrix models [113]. Interestingly it has been shown that the tails of the time delay distribution follow a universal power law [98, 99, 100, 104, 105, 109, 114]. Several methods has been applied in order to understand the properties of the delay time. A path integral approach has been applied for the analysis of delay time for wave packet scattering [115]. It has been shown that an imaginary potential can be a paradigm in connecting the reflection coefficients to the distribution of time delay [116]. In some cases Random Matrix theory has been applied to understand the dynamics when there is broken time-reversal symmetry [117]. But more interestingly a 
general class of Fokker-Planck Equation of exponential functionals of Brownian motion with drift have been implemented [97]. For the study of delay time distribution in our system of a random waveguide with corrugated surfaces (figure 3.1), we will implement a probabilistic approach in order to understand the nature of the probability distribution of time delays. For our model we will treat the electrons as classical objects with no interaction among them. Because of the presence of a transversal magnetic field perpendicular to the plane of the model, some of the electrons will get transmitted and some of them will get reflected. We know from the previous chapter that the conductance in one direction is determined fully by the characteristics of only the chaotic trajectories (from left to right), while the transmission in the other direction contains a mixture of chaotic as well as regular skipping orbits. Also in the limit of the system length $L \rightarrow \infty$ the characteristic transmission from the right to the left is mainly governed by the dynamics of regular trajectories. Therefore, it is interesting to note that the characteristic time distributions must be different for transmission in left to right direction, from the opposite direction. In the former case the dynamics is fully chaotic, whereas in the latter case the dynamics is mainly regular (when $L \rightarrow \infty$ ). We will use the probabilistic approach in order to find the behavior of time delay in both these cases of transport. Some of the aspects of this approach are discussed below.

In probabilistic models time delays have been treated as First-Passage Problems. The problem is based on a few basic questions associated with the systems. Some of them are, given a system with initial conditions

- what is the probability that a diffusing particle hits one of the boundary points of the system for the first time at time $t$ ?

- What is the probability that the particle has not hit the boundary by time $t$ ?

- How long does it take to hit one of the boundary points?

The first question deals with finding the first-passage characteristics of the system. The second question addresses the survival probability in an open system; while the third one deals with the average time (more general, the moments of the first-passage probability) taken by a particle before it can hit the boundary. As the survival probability and the average mean time can be related to the first-passage probability (FPP), we only need to calculate the first-passage probability for the electron inside the waveguide. For our calculations, we assume absorbing boundary conditions at both the ends of our system. The reason is, a particle getting into the system must come out of the channel at one of the either end points of the channel; once it comes out of the channel it no longer remains a part of the process. We know that the chaotic trajectories has a diffusive behavior about the mean value. In the previous chapters we are able to find a characteristic density distribution for the occupational probabilities of the electrons. We also have seen that for large enough system lengths the dynamics of transmission can be treated in terms of a biased diffusion equation. So, for the time being we will 
consider length scales of the system such that $L>>\lambda_{\mathrm{ch}}$ i.e., the system size is such that the momentum correlations has already vanished in the chaotic part of the trajectories and the distribution is essentially Gaussian. We can use this fact to calculate the delay time distribution. But first, let us relate the First Passage Probability (FPP) to the characteristic occupational probability of a system.

\subsection{Connection between FPP and the probability density}

In the previous chapters, we have studied the dynamics of an electron inside the waveguide with a disordered boundary using Markov chains. Therefore it is sufficient to treat a random walk inside the channel with average spacing $\Delta x(\phi)$ and average time $\Delta l(\phi) / v_{0}$, where $\phi$ is the angle of scattering at the disordered boundary with the probability of scattering at an angle $\phi$ being $P(\phi)=(1 / 2) \sin \phi$. These relations and the corresponding average chaotic velocity and an associated diffusion constant has been calculated in the previous chapters and are provided in equations (3.6), (3.7), (3.20) and (3.30). Using these, we have shown that the occupational probability of an electron inside an infinite waveguide is a Gaussian peaked at the position of mean velocity times the time.

So, for the connection between the FPP and the occupational probability of the particle, we reconsider the same random walk and define $P(x, t)$ as the occupational probability; i.e., the probability that a random walk is at site $x$ at time $t$. Similarly we define $F(x, t)$ be the FPP, namely the probability that the random walk visits $x$ for the first time at time $t$, both $P(x, t)$ and $F(x, t)$ being obtained with the same initial conditions for the particle. Once a particle reaches a point $x$ from its starting point, there is no further contribution to the FPP $F(x, t)$, although the same might contribute to the occupational probability $P(x, t)$. Therefore, it is clear that $F(x, t)$ vanishes much more rapidly (as a function of time) than the counterpart occupational probability $P(x, t)$. Moreover, for a random walk to be at $x$ at time $t$, the walker must first reach $x$ at some earlier step $t^{\prime}$ and then return to $x$ after an additional time $t-t^{\prime}$

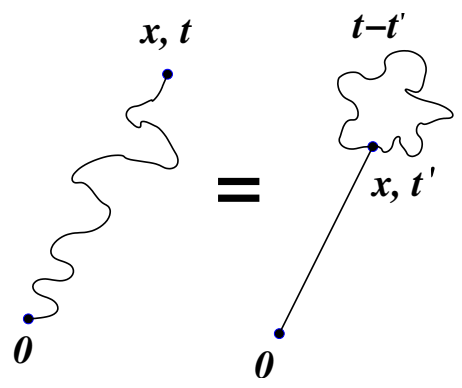

Figure 5.1: Diagrammatic relation between the occupational probability of a random walk (curved line) and the first passage probability (straight line). For the particle to be at $x$ at time $t$, it must reach $x$ at an earlier time $t^{\prime}$ and then return to the same position after an additional time of $t-t^{\prime}$. 
steps. This is illustrated in figure 5.1. This helps to write the connection between $F(x, t)$ and $P(x, t)$ as

$$
P(x, t)=\sum_{t^{\prime} \leqslant t} F\left(x, t^{\prime}\right) P\left(0, t-t^{\prime}\right) .
$$

For continuous in space and -time processes we can rewrite this equation (5.1) in the form of an integral

$$
P(x, t)=\int_{0}^{t} F\left(x, t^{\prime}\right) P\left(0, t-t^{\prime}\right) d t^{\prime} .
$$

As the Laplace convolution of two functions $g$ and $h$ is given by

$$
g(t) * h(t)=\int_{0}^{t} g\left(t-t^{\prime}\right) h\left(t^{\prime}\right) d t^{\prime},
$$

and the Laplace transform of the convolution is the multiplication of the individual Laplace transforms, we perform a Laplace transform of equation (5.2), by multiplying both sides with $e^{-s t}$ and integrating from 0 to $\infty$ over the time variable $t$, to obtain the simpler form

$$
P(x, s)=F(x, s) P(0, s),
$$

where

$$
\begin{aligned}
P(x, s) & =\int_{0}^{\infty} e^{-s t} P(x, t) d t=\mathcal{L}(P(x, t)), \\
\text { and } F(x, s) & =\int_{0}^{\infty} e^{-s t} F(x, t) d t=\mathcal{L}(F(x, t)) .
\end{aligned}
$$

are the Laplace transforms of the probabilities $P(x, t)$ and $F(x, t)$ respectively. Using equation (5.3) we can obtain the FPP $F(x, t)$ by inverting the Laplace transform

$$
F(x, t)=\mathcal{L}^{-1}\left(\frac{P(x, s)}{P(0, s)}\right) .
$$

Here we want to point out that the Laplace transform contains the information about all moments of the FPP distribution. This can be seen as follows. We know that the Laplace transform is

$$
F(x, s)=\int_{0}^{\infty} e^{-s t} F(x, t) d t .
$$

Differentiating $F(x, s), n$ times with respect to $s$ and evaluating the integral at $s=0$, yields

$$
\left.(-1)^{n} \frac{\partial^{n}}{\partial s^{n}} F(x, s)\right|_{s=0}=\int_{0}^{\infty} t^{n} F(x, t) d t=\left\langle t^{n}\right\rangle .
$$


In other words, the moments of the first-passage probability distribution are the Taylor coefficients of the Laplace transform at $s=0$, multiplied by $(-1)^{n}$. This provides the answer to the third question, namely the mean passage time for the diffusing particle. We will use this information (of Laplace transformations) and the informations obtained in the previous chapters to calculate the delay time probability for an electron inside the waveguide with a disordered boundary.

\subsection{Distribution of delay times for the finite channel}

We have obtained the probability distribution, therefore we can perform a Laplace transform over it and then find the solution; but we are not implementing that! Instead we calculate the distribution of delay times by first Laplace transforming the equation and then inverting it. The reason will be clear once we apply this formalism to the electronic setup of our system. To solve for FPP, we assume that the biased diffusing particle initiates its motion at some position $x=x_{0}\left(x_{0} \gg \lambda_{\mathrm{ch}}\right.$ (the reason is, we assume the situation where the particle has already diffused into the system so that the Gaussian approximation for the probability distribution $P(x, t)$ is valid) and it gets absorbed when it hits either ends of the boundaries. Therefore, we need to solve the convection-diffusion equation

$$
\frac{\partial P(x, t)}{\partial t}+v \frac{\partial P(x, t)}{\partial x}=\frac{D}{2} \frac{\partial^{2} P(x, t)}{\partial x^{2}}
$$

subject to the initial condition $P(x, t=0)=\delta\left(x-x_{0}\right)$ and the boundary conditions $P(x, 0)=P(x, L)=0$, where $L$ is the length of the finite channel.

In the derivation following [74], we can write the solution for the Laplace transform of the concentration for this problem in the entire domain as

$$
P(x, s)=\frac{2 e^{v\left(x-x_{0}\right) / D}}{D w(s) \sinh [w(s) L]} \sinh \left[w(s) x_{<}\right] \sinh \left[w(s)\left(L-x_{>}\right)\right] .
$$

where $x_{>}=\max \left(x, x_{0}\right)$ and $x_{<}=\min \left(x, x_{0}\right)$. See Appendix $\square$ for details of the steps followed. Using this we can calculate the flux (or the Laplace transform of the FPP) to $x=L$

$$
j_{+}\left(x_{0}, s\right)=v P_{>}(x, s)-\left.\frac{D}{2} \frac{\partial P_{>}(x, s)}{\partial x}\right|_{x=L}=e^{v\left(L-x_{0}\right) / D} \frac{\sinh \left[w(s) x_{0}\right]}{\sinh [w(s) L]} .
$$

Clearly in case of an electronic setup (of our model) the transmission from left to right is only by chaotic trajectories whereas on the other direction the transmission consists of both regular skipping orbits and chaotic trajectories. We concentrate on the chaotic transmission from left to right. Then for an electron to pass from $x=0$ to $x=L$ within a time $t$ for the first time, it should pass from $0 \rightarrow x_{0}$ for the first time within a time $t^{\prime}<t$ and then starting from $x_{0}$ it should transmit to $x=L$. Based on this argument we can write a recursion relation for the distribution of delay times of an electron entering at 


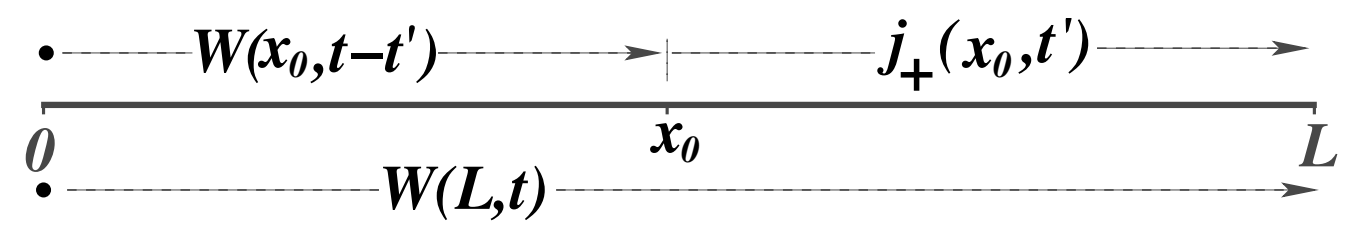

Figure 5.2: For the electron entering at $x=0$ and exiting at $x=L$, the particle should pass through the intermediate point $x_{0}$ within the delay time and then exit from $x=x_{0}$ to $x=L$ within the first passage probability. This provides a recursion relation between the delay time distributions between the points $x=x_{0}$ and $x=L$ which is shown in equation (5.10).

$x=0$ and exiting at $x=L$

$$
W(L, t)=\int_{0}^{t} W\left(x_{0}, t-t^{\prime}\right) j_{+}\left(x_{0}, t^{\prime}\right) d t^{\prime} .
$$

This equation will be valid as long as we chose the position $x_{0}$ in such a way that the momentum correlations have no effect on it. This is ensured by the assumption $x_{0} \gg \lambda_{\mathrm{ch}}$. Since the right hand of the integral is a convolution, the Laplace transform of the above equation easily provides

$$
W(L, s)=W\left(x_{0}, s\right) j_{+}\left(x_{0}, s\right) .
$$

where

$$
\begin{aligned}
W(L, s) & =\int_{0}^{\infty} e^{-s t} W(L, t) d t \\
\text { and } j_{+}\left(x_{0}, s\right) & =\int_{0}^{\infty} e^{-s t} j_{+}\left(x_{0}, t\right) d t .
\end{aligned}
$$

We already have calculated, in equation (5.9), that within the FPE the FPP to the exit in the direction of $x=L$ for a particle starting from a point $x=x_{0}$ is $j_{+}\left(x_{0}, s\right)$. On substitution we find

$$
W(L, s)=W\left(x_{0}, s\right) e^{v\left(L-x_{0}\right) / D} \frac{\sinh \left[w(s) x_{0}\right]}{\sinh [w(s) L]}
$$

which has a solution

$$
W(L, s)=C \frac{e^{v L / D}}{\sinh [w(s) L]} .
$$

where $C$ is a constant and can be determined from the normalization of the delay time distribution $W(L, t)$. Analytically the inverse Laplace transform for the above equation is not that easy, so we assume a large $L$ limit of the system $L \rightarrow \infty$. Then we can approximate the above equation

$$
W(L, s) \approx C e^{v L / D} \exp [-w(s) L]=C \exp \left[\frac{\left(v-\sqrt{v^{2}+2 D s}\right) L}{D}\right], \quad(L \rightarrow \infty)
$$




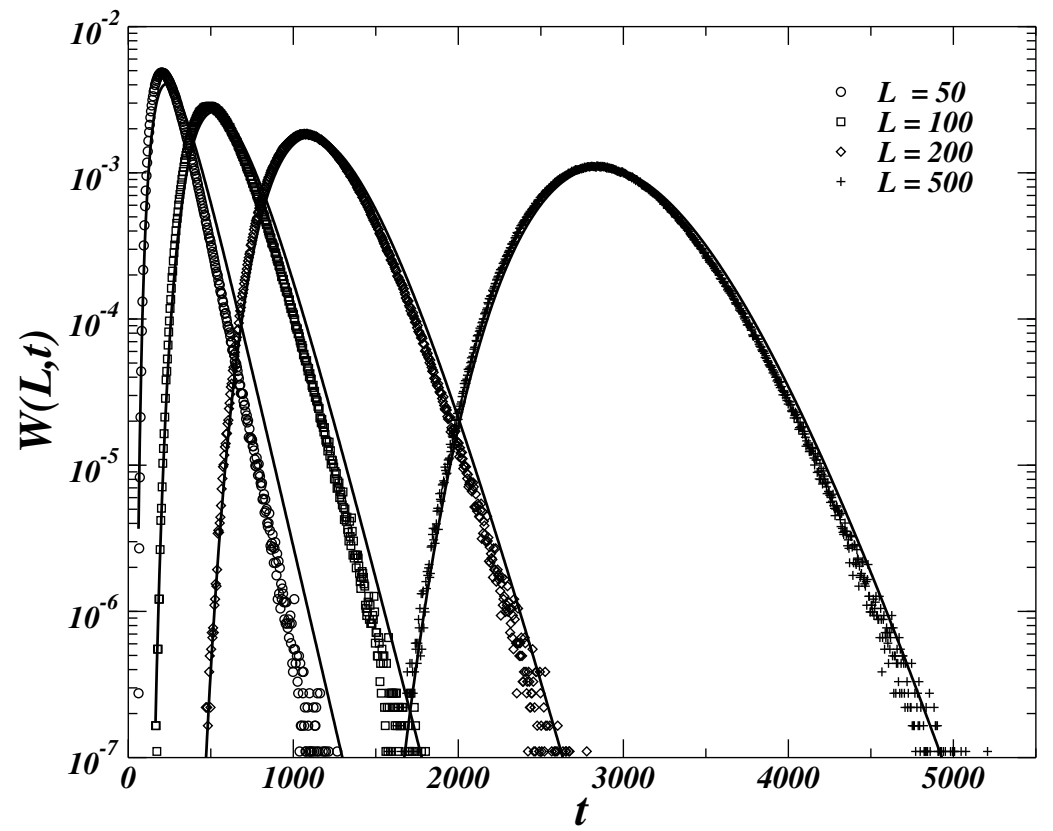

Figure 5.3: The prediction of the equation (5.15) (plotted with lines) has been compared with the results of the transmission delay time distribution of the numerical simulation (plotted with symbols) obtained by simulating $N=10^{7}$ trajectories entering at $x=0$ equally distributed in the phase space i.e., with probability $P(\phi)=(1 / 2) \cos \phi,(\phi \in[-\pi / 2: \pi / 2])$ and $P(y)=1,(y \in[0,1])$. The value of cyclotron radius used for the simulation is $r_{c}=4 b$ and $b=1$.

after substitution of $w(s)$. Performing an inverse Laplace transform provides

$$
W(L, t)=\frac{L}{\sqrt{2 \pi D t^{3}}} \exp \left[-\frac{(L-v t)^{2}}{2 D t}\right]
$$

after normalization. Clearly the distribution is centered about the mean value of position. It is a consequence of directedness of chaotic orbits. The results of this prediction are shown in figure 5.3 for various lengths of the system. The results very well match the predictions of the equation. However we can observe systematic deviations when the length of the system is small (see the tails of the distribution when $L$ is small). The obvious reason is that the approximation of $L \rightarrow \infty$ does not hold true in this regime. To get a better understanding of these deviations, we do a scaling of the distribution which we discuss below.

\subsubsection{Scaling of the Delay time distribution}

In order to scale the distribution, so that different distributions can be compared on a single basis, we change the variable from time $t$ to a dimensionless variable $\tau=t(v / L)$ and note that the probability 
distribution is normalized to unity

$$
\int_{0}^{\infty} W(L, t) d t=\int_{0}^{\infty} \frac{L}{\sqrt{2 \pi D t^{3}}} \exp \left[-\frac{(L-v t)^{2}}{2 D t}\right] d t=1 .
$$

Now replacing the time $t$ with the dimensionless variable $\tau$ we obtain

$$
\int_{0}^{\infty} W(L, t) d t=\int_{0}^{\infty} W_{s}(L, \tau) d \tau=\int_{0}^{\infty} \sqrt{\frac{L v}{2 \pi D \tau^{3}}} \exp \left[-L v \frac{(1-\tau)^{2}}{2 D \tau}\right] d \tau=1 .
$$

This provides the scaled probability distribution

$$
W_{s}(L, \tau)=\sqrt{\frac{L v}{2 \pi D \tau^{3}}} \exp \left[-L v \frac{(1-\tau)^{2}}{2 D \tau}\right] .
$$

The results of this scaling are shown in figure 5.4. The figure clearly shows the deviations of the

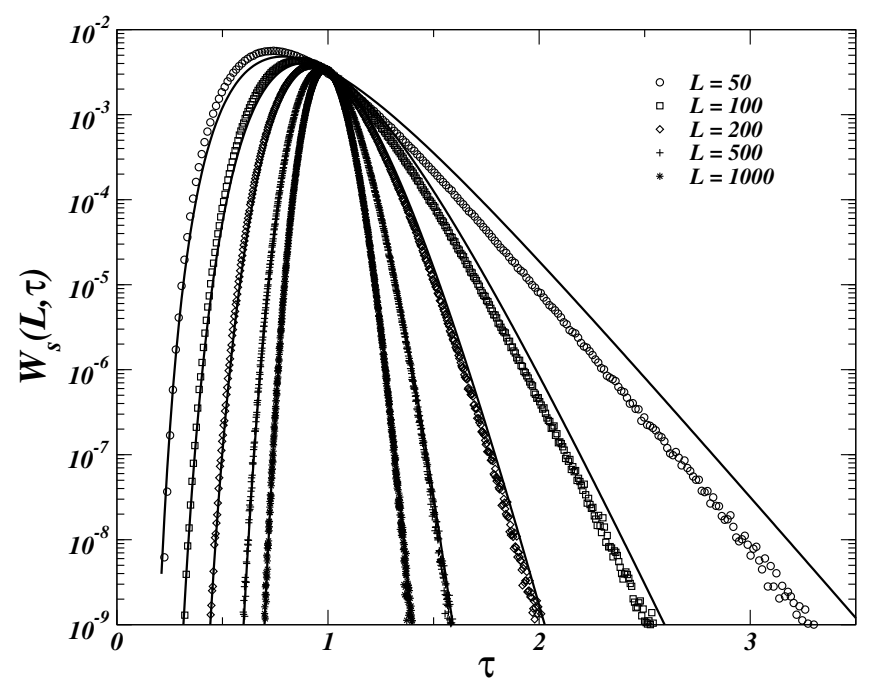

Figure 5.4: The results for the scaled distribution (5.18) (plotted using lines) for the transmission delay times has been compared with the results of the numerical simulation (in symbols). The conditions used for the numerical distribution are same as in figure (5.3).

prediction for smaller length scales of the system (even at $L=100$ we can observe deviations for larger time scales). However as the system size increases the results agree very well with the predictions (compare the result for $L=500$ or 1000). In order to understand qualitatively the results for the deviations at larger time scales and smaller length scales of the system we expand the results of equation 
(5.13) for small value of $s$ (in other words large values of $t$ ). This yields

$$
W(L, s) \sim \frac{\exp \left(\frac{L v}{D}\right)}{\sinh \left(\frac{L v}{D}\right)+s \frac{L}{v} \cosh \left(\frac{L v}{D}\right)}
$$

whose inverse Laplace transform can be written in the form

$$
W(L, t) \propto \frac{v}{L}\left[1+\tanh \left(\frac{L v}{D}\right)\right] \exp \left[-\frac{t v}{L} \tanh \left(\frac{L v}{D}\right)\right] .
$$

This can be written in the form

$$
W(L, t)=\alpha \exp (-\mu t) .
$$

where $\alpha$ and $\mu$ are constants which depend on the parameters of the system. The results of the distribution from numerical simulations has been fitted to this equation for larger values of time and are shown in figure 5.5 for various system lengths. As can be seen they agree very much with the expected

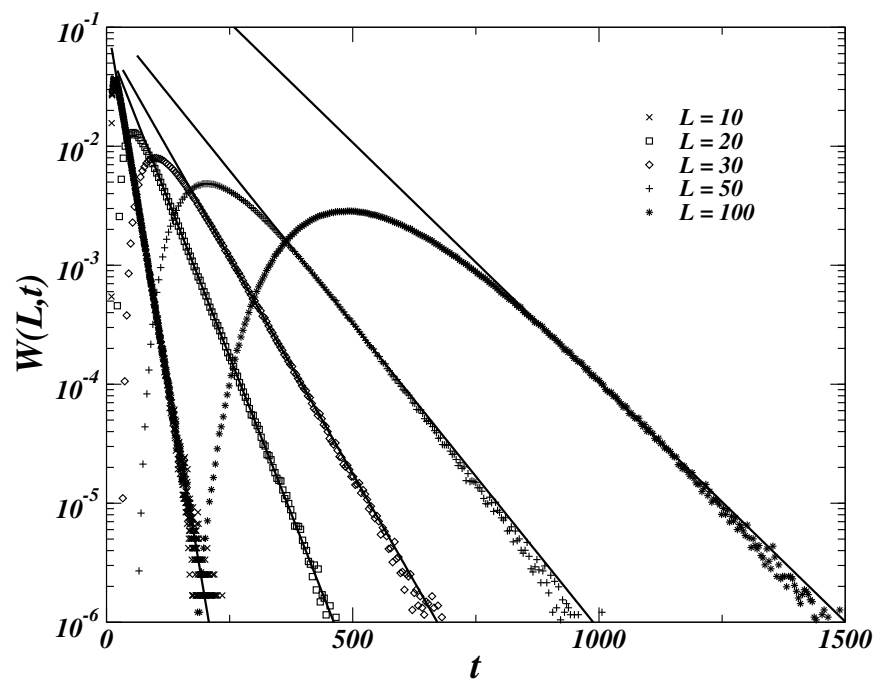

Figure 5.5: The delay time distribution (symbols) has been plotted against an exponential fitted function (lines) as in equation (5.21). The results clearly shows the exponential nature of the delay time distribution for large values of $t$. The fitted exponential function agrees with numerical results especially for smaller values of $L$ and for $t>L / v_{\mathrm{ch}}$.

results (especially for smaller values of $L$ and for $t>L / v_{\mathrm{ch}}$ ). However there is disagreement between the value of $\mu$ obtained from the fitted value with the analytical value in equation (5.20). The analytical prediction says that

$$
\mu=\frac{v}{L} \tanh \left(\frac{L v}{D}\right) .
$$


For a comparison of these results see table (5.1). The reason for these discrepancies are not clear, but the corrections can be fitted to a curve $f(L)=\alpha+\beta / L^{2}$. Nevertheless there are much more interesting results in the reflection properties, which we are discussing below.

\begin{tabular}{c|c|c}
\hline Length & Analytical & Fitted \\
\hline \hline 10 & 0.0143945 & 0.05623 \\
20 & 0.00839025 & 0.02428 \\
30 & 0.00566538 & 0.01673 \\
50 & 0.00340315 & 0.01184 \\
100 & 0.00170159 & 0.00927 \\
\hline \hline
\end{tabular}

Table 5.1: Comparison of the values of $\mu$ obtained from expression (5.22) with the fitted value.

\subsection{Distribution of Reflection Time Delays}

We have seen the characteristic nature of the transmission time reflecting the directedness of the chaotic trajectories. On the other hand, as the length of the system grows $L \rightarrow \infty$, almost none of the chaotic trajectories move against the direction of drift. Therefore, for large system lengths the delay time distribution of the reflected trajectories should remain independent of the length of the system. This is observed in figure 5.6. As the trajectories get reflected in the same direction they entered, the quantitative nature of the time delay can't be based on the transmission of chaotic trajectories.

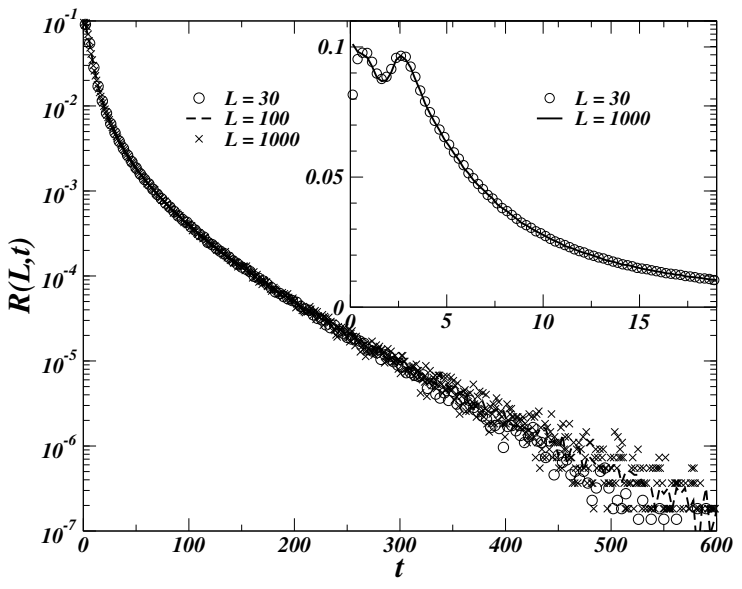

Figure 5.6: The distribution of reflection delay times for various lengths of the system $L=30,100$ and $L=1000$. It shows that the distribution is independent of the length of the system for large values of system length $L$. Inset shows that the distribution initially increases for a short while and then decreases. 
Nevertheless we can provide some qualitative arguments which will provide some insight into the basic nature of the distribution.

If the particles are entering from left then because of momentum correlations the electrons will move relatively at a faster speed for a certain time. Let $x_{0}$ be the length the electron needs to travel before we can neglect these correlations, $t_{1}$ be the time scale for the particle to travel this length $x_{0}$ and $t_{2}$ be the time scale taken by the particles to get reflected from this point to the absorbing wall at $x=0$. Clearly $t_{2} \gg t_{1}$. This is because the trajectories moving against the drift take a larger time to travel the length $x_{0}$ than the time taken by the particles to travel along the drift. With this argument we can assume that the total time taken by the electrons to reach the boundary at $x=0$ (the point at which they entered) to be $t=t_{1}+t_{2} \approx t_{2}$. Therefore, we can neglect the time $t_{1}$ and then find out the probability of a particle to diffuse from a point $x_{0}$ and get absorbed at the absorbing boundary at $x=0$. This is exactly $j_{-}\left(x_{0}, t\right)$, the flux to $x=0$ for an electron starting from the point $x=x_{0}$, which can be calculated from the expression of $P_{<}(x, s)$ provided in equation (5.8)

$$
\begin{aligned}
j_{-}\left(x_{0}, s\right) & =-v P_{<}(x, s)+\left.\frac{D}{2} \frac{\partial P_{<}(x, s)}{\partial x}\right|_{x=0} \\
& =\exp \left(-\frac{v x_{0}}{D}\right) \frac{\sinh \left[w(s)\left(L-x_{0}\right]\right.}{\sinh [L w(s)]} \\
& =\exp \left(-\frac{v x_{0}}{D}\right)\left\{\cosh \left[w(s) \lambda_{\mathrm{ch}}\right]-\sinh \left[w(s) x_{0}\right] \operatorname{coth}[w(s) L]\right\}
\end{aligned}
$$

Since we are interested in large length scales of the system $L \rightarrow \infty$ for which $\operatorname{coth}[w(s) L] \simeq 1$, we obtain

$$
j_{-}\left(x_{0}, s\right) \approx \exp \left(-\frac{v x_{0}}{D}\right) \exp \left[w(s) x_{0}\right]=\exp \left[\frac{-\left(v-\sqrt{v^{2}+2 D s}\right) x_{0}}{D}\right] .
$$

Inverse Laplace transform provides us the value for the reflection delay time distribution $R(L, t)$ as

$$
R(L, t)=\frac{x_{0}}{\sqrt{2 \pi D t^{3}}} \exp \left[-\frac{\left(x_{0}+v t\right)^{2}}{2 D t}\right] .
$$

So, we expect that the distribution of reflection time delay will follow

$$
R(L, t) \propto t^{-3 / 2} \exp \left(-\beta t-\frac{\gamma}{t}\right) .
$$

with $\beta \approx v^{2} /(2 D)=v /\left(4 \lambda_{\mathrm{ch}}\right)$ and $\gamma=x_{0}^{2} /(2 D)$. The results are shown in figure 5.7 for various values of cyclotron radius. This shows that there is very much agreement between the data and the predictions. None the less there are discrepancies for very short time scales.

We have assumed that the total time is equivalent to the total diffusive time. Also the reflecting electrons need to move against the actual drift motion of the chaotic electrons in order to get reflected. 

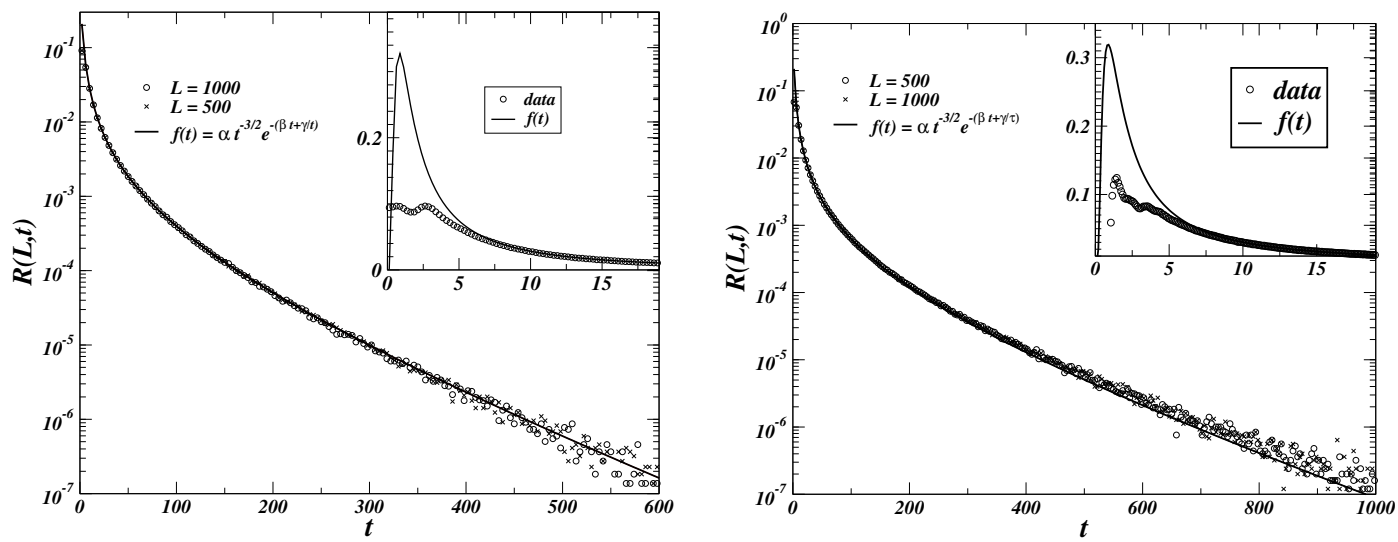

Figure 5.7: The delay time distribution of reflected trajectories for $r_{c}=4$ (left) and $r_{c}=6$ (right) fitted against the function (5.26). The values of $\alpha, \beta$ and $\gamma$ are provided in table 5.2. The inset shows that the fitted function can not reproduce the results for very short times.

\begin{tabular}{c|c|c|c|c|c}
\hline$r_{c}$ & $\alpha$ & $\beta$ & $\gamma$ & $v^{2} /(2 D)$ & $x_{0}=\sqrt{2 D \gamma}$ \\
\hline \hline 4 & 1.12224 & 0.01025 & 1.24661 & 0.010564 & 1.848406 \\
6 & 1.15679 & 0.00603 & 1.29647 & 0.005713 & 2.052475 \\
8 & 1.12955 & 0.00382 & 1.31517 & 0.003763 & 2.178581 \\
10 & 1.12563 & 0.00278 & 1.35209 & 0.002746 & 2.292484 \\
15 & 1.13845 & 0.00162 & 1.9506 & 0.001576 & 2.926563 \\
\hline \hline
\end{tabular}

Table 5.2: Values of $\alpha, \beta$ and $\gamma$ and the analytical value of $v^{2} /(2 D)$ for various values of cyclotron radius. The table also provides an estimation for the value of $x_{0}$.

So, they have an average velocity which is negative. Therefore the probability should start decreasing right from the point where $x_{0}$ is located. So, the position of the initial peak in the distribution will provide the information about the value of $x_{0}$. From the fitted curve we can observe that the value of $\gamma$ is not good enough to reproduce the exact value of $x_{0}$ or the initial peak in the distribution. In contrast we find that, for larger values of $r_{c}$, the numerical values of the first peak almost remains a constant $x_{0} \approx 1.5$. Nevertheless the value of $\beta$ nearly matches the analytical value of $v^{2} /(2 D)$. The reason is following. As the time $t$ increases the approximation $t \approx t_{2}$ becomes more and more realistic, but for small times the error shows up. Therefore, the value of $x_{0}$, being a small time factor, can not be obtained from the numerical value of $\gamma$ (which has been obtained in the approximation of $t_{2} \gg t_{1}$ ). To summarize the results, we are able to understand quantitatively and qualitatively how the delay time distribution of the chaotic trajectories will behave in a system supporting directed chaos. There is still one more question remained. What about the regular trajectories? We know that the regular trajectories never get backscattered and always move in a single direction. We also know that the transmission 
probability for these regular skipping orbits in the direction of their travel is always unity. So it will be really interesting to have a look at their time delays. In the second chapter where we introduced our toy models, we already have encountered some divergences in the velocity distribution (for a finite system) of these trajectories. Therefore, we hope the same effect should be observed in the delay time properties of these regular skipping orbits.

\subsection{Delay Time Distribution of the Regular Skipping Orbits}

Since the regular skipping orbits never get back-scattered the study of the delay time distribution of these trajectories means we are dealing with the transmission delay properties of them. As in the delay time properties of the chaotic trajectories reflected the directedness of the chaotic trajectories, we expect the regular trajectories to show divergences in the distribution. Indeed if we take a look at the delay time distribution of the regular skipping orbits, we can observe the divergences reappearing (see figure 5.8).
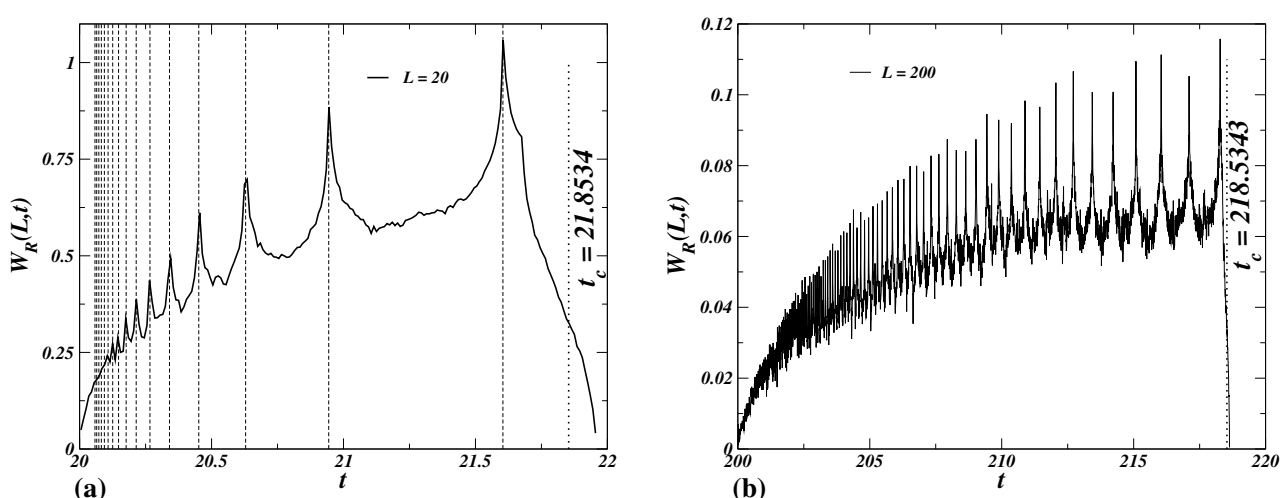

Figure 5.8: The delay time distribution of the regular skipping orbits show regular occurrence of divergences at some particular times marked by the vertical dashed line in (a) for the length of the system $L=20$. The number of these peaks gets increased in number as we go to higher lengths of the system size as shown in (b) for $L=200$. The distribution remains bounded within two time scales one equal to the length of the system and another $t_{c}$ provided by the average value of the critical value of velocity beyond which the trajectory becomes chaotic. The value of cyclotron radius chosen for these graphs is $r_{c}=4 b$, while the number of initial trajectories $N=10^{7}$ are equally distributed in the region of negative velocity of the Poincaré section. For clarity reasons we have discarded the chaotic tail in (a).

However for small length scales there will be a contribution of the chaotic trajectories moving from right to left to the time delay of electrons. But the time scale of occurring these chaotic distributions will be more than the time scales at which the regular skipping orbits occur. In other words there is a clear separation between the time at which the regular skipping orbits transmit and the time at which the chaotic orbits transmit from right to the left end of the channel. This feature of chaotic and regular 


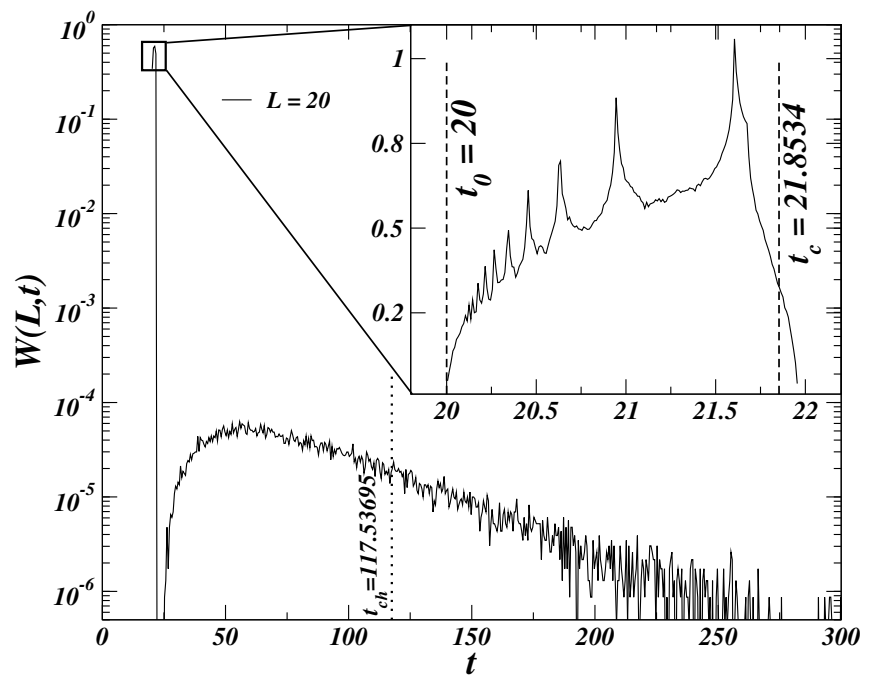

Figure 5.9: For shorter system lengths, the distribution of delay times of electrons, moving from right to left of the channel, will show a chaotic tail. However the probability of chaotic trajectories getting transmitted against the drift decreases with the increase in the length of the system. The inset shows the distribution of only the regular skipping orbits (plotted in a different scale). As can be seen the distribution of regular skipping orbits is enclosed within two time scales $t_{0}$ and $t_{c}$ (see the text for details). The average chaotic time $t_{\mathrm{ch}}$ is shown with a vertical dotted line.

orbits is reflected in figure 5.9. Calculating the time scales involved with this separation, of chaotic orbits from the regular trajectories, will provide the upper bound of the regular delay time distribution. The details of these calculations are discussed in the following section.

\subsubsection{Time Scales within the Distribution}

We start a trajectory at $(x=0, y, \varphi)$ with $\varphi \in[\pi / 2,3 \pi / 2]$. For this trajectory to be a regular skipping orbit it should satisfy the following condition (see figure 5.10)

$$
\varphi_{\min }(y) \leqslant \varphi \leqslant \varphi_{\max }(y),
$$

otherwise the trajectory becomes chaotic. The equalities on the both the ends will mean that the lower boundary becomes a tangent to the trajectory. We can call $\varphi_{\min }(y)$ and $\varphi_{\max }(y)$ the minimum and the maximum possible angles at $(x, y)$ such that a trajectory always remains a regular skipping orbit. These bounds of the angle hold the clue to the minimum and the maximum limit of the time scales involved with the time delay distribution of the regular trajectories. Looking at these regular trajectories, they require that the current arc's center to remain a distance $r_{c}$ away from the disordered lower boundary. Using this the expression of these bounded values of $\varphi$ has already been calculated in equation 2.36 


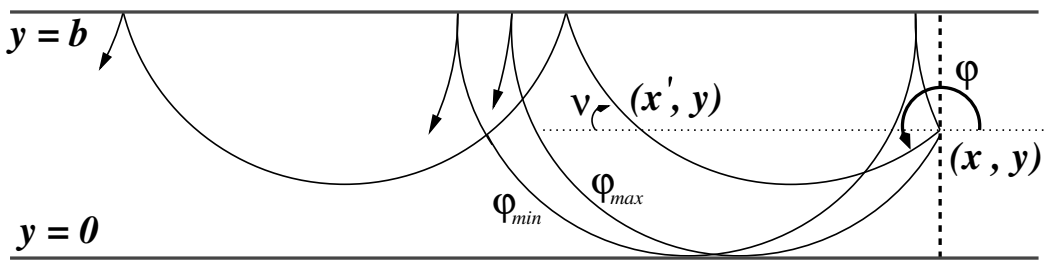

Figure 5.10: Any trajectory starting at any point $(x, y, \varphi)$ with $\varphi \in[\pi / 2,3 \pi / 2]$ to be a regular skipping orbit should follow the condition in equation (5.27). If the angle is such that $\varphi<\varphi_{\min }$ or $\varphi>\varphi_{\max }$, then the trajectory gets reflected randomly from the lower boundary making it a part of chaotic trajectories.

(we recover our results by substituting $R=0$ in the expression of $\alpha(y)$ provided in equation (2.35)). However things are different at the end points (i.e., when we have $y=0$ or $y=b$ ) along the vertical axis. At $y=0$ the only possible allowed angle is

$$
\pi \leqslant \varphi \leqslant \pi
$$

whereas at $y=b$, the allowed ranges for the angle $\phi$ to be regular skipping is

$$
\pi<\phi \leqslant \pi+\arccos \left(1-\frac{b}{r_{c}}\right) .
$$

Based on this information we have calculated that the average velocities $v_{\text {reg }}$ of all the regular skipping orbits will satisfy

$$
-1 \leqslant v_{\text {reg }} \leqslant-\frac{\sqrt{b\left(2 r_{c}-b\right)}}{r_{c} \arccos \left(1-\frac{b}{r_{c}}\right)} .
$$

For details of these derivations see the section 2.4. Hence the time taken by the regular skipping orbits through a channel of length $L$ in the negative direction will be within the range

$$
L \leqslant t \leqslant t_{c},
$$

where $t_{c}$ is given by

$$
t_{c}=\frac{L}{\frac{\sqrt{b\left(2 r_{c}-b\right)}}{r_{c} \arccos \left(1-\frac{b}{r_{c}}\right)}}=\frac{L r_{c} \arccos \left(1-\frac{b}{r_{c}}\right)}{\sqrt{b\left(2 r_{c}-b\right)}} .
$$

The value of $t_{c}$ can be recognized as the average time taken by the trajectory which has the rough boundary as its tangent and transmits a length of $L$ in the negative direction. In the inset of figure 5.9 these two values have been marked with two dashed lines at $t_{0}=L$ and at $t_{c}$. We find there is some small probability of regular trajectories having time greater than $t_{c}$. The reason is following. In performing 
the calculations by mapping a point $(x=0, y, \varphi)$ to the point $\left(x^{\prime}, y=b, \phi\right)$, we have discarded the initial displacement. By discarding this initial displacement we make a small error which for $L \rightarrow \infty$ approaches zero. Thus, this type of errors are expected in the distributions showing some probabilities beyond $t_{c}$. This effect gets enhanced for smaller lengths of the system.

On the other hand the chaotic trajectories have a mean velocity in the opposite direction of the regular ones. This balances the total current inside the system. Moreover, they get reflected randomly from the lower boundary $(y=0)$ with a probability distribution $P(\phi)=(1 / 2) \sin \phi$. As a result of the motion against the drift velocity and because of random scattering the chaotic trajectories stay longer inside the channel. We also can prove it quantitatively based on the sum rule

$$
\begin{aligned}
\Omega_{\mathrm{reg}} v_{\text {reg }}+\Omega_{\mathrm{ch}} v_{\mathrm{ch}} & =0, \\
\Rightarrow-\frac{v_{\mathrm{ch}}}{v_{\text {reg }}} & =\frac{\Omega_{\mathrm{reg}}}{\Omega_{\mathrm{ch}}} .
\end{aligned}
$$

If $r_{c}>b$ i.e., when the magnetic field is of moderate strength (to remind you, our calculations are based on this regime of magnetic field), the chaotic trajectories remain in both panels of the Poincaré section; whereas the regular ones remain only in a single direction (see figure 3.2). Therefore,

$$
\begin{aligned}
\frac{\Omega_{\mathrm{reg}}}{\Omega_{\mathrm{ch}}} & <1 \\
\Rightarrow \quad v_{\mathrm{ch}} & <-v_{\text {reg }},
\end{aligned}
$$

using equation (5.33). Hence, the average time taken by the chaotic trajectories to transmit through the channel with a disordered boundary of length $L$ will be greater than the time taken by the regular trajectories to transmit through the same length of the channel in the opposite direction. The chaotic time scale

$$
t_{c h}=\frac{L}{v_{\mathrm{ch}}}
$$

is provided with a dotted line in figure 5.9. Apart from these time scales, we can observe peaks in the delay time distribution of the regular skipping trajectories. Our system does not have any pulsating behavior. Therefore, at first instant the system has nothing to do with the occurrence of these peaks in the distribution. Instead the peaks has something to do with the way the electrons travel through the finite channel. To complete the picture we need to know the time taken by a trajectory entering at $x=0$ and exiting at $x=-L$. Initially we describe this dynamics (of time) in a simpler way by assuming that the trajectories has no separation along the vertical axis and they start at the point $(x=0, y=b)$ with an angle $\phi$ satisfying the condition (5.29). Then we will describe the dynamics in terms of electrons entering at any point $(x=0, y, \varphi)$ with $\varphi$ satisfying the condition (5.27). The following section deals with the special set of trajectories entering with an initial point $(x=0, y=b, \phi)$. 


\subsubsection{Special set of trajectories entering at $(0, b, \phi)$}

The reason for assuming these trajectories to be special will be clear only when we will discuss the general case of any trajectory entering at $(0, y, \varphi)$. Nevertheless the dynamics of these special trajectories entering at $(0, b, \phi)$ is simple to describe as we have only one parameter to vary namely the angle $\phi$ which has an upper and a lower bound provided in equation (5.29). A typical trajectory with this initial condition which transmits through the finite channel of length $L$ is shown in figure 5.11. From this diagram, we can easily observe that the electron entering at $(0, b, \phi)$ in order to transmit

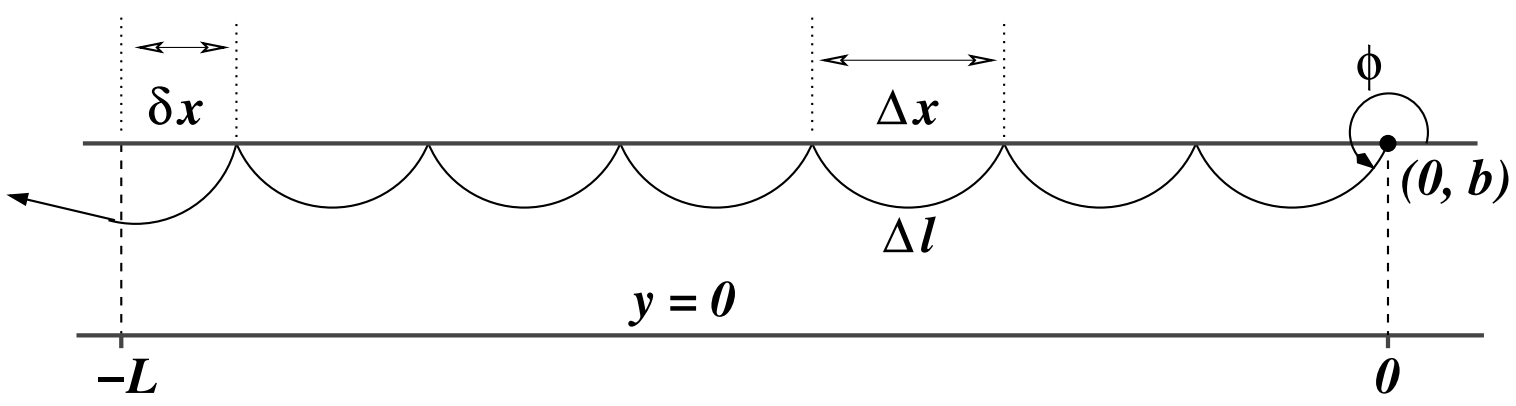

Figure 5.11: A regular trajectory entering the channel at $(0, b, \phi)$ will eventually have to cross the entire length $L$ of the finite channel. Depending on the initial angle $\phi$, the trajectory can exactly fit into the entire length of the channel with an integer number of equal sections each of length $\Delta x$ (in which case the final section will have length zero) or it may not (leaving a remainder section of length $\delta x$ ). Accordingly the time will depend on $\Delta x$ and $\delta x$. See the text for details.

through the finite channel of length $L$ has to span an integer (say $m$ ) number of equal lateral sections each of length $\Delta x=2 r_{c} \sin \phi$ and the rest amount given by

$$
\delta x=L-m|\Delta x| \quad(0 \leqslant \delta x<|\Delta x|)
$$

Therefore, the total length $L$ of the finite channel can be written as

$$
L=2 m r_{c}|\sin \phi|+\delta x .
$$

So, the total time taken by the trajectory can be written as

$$
t(L, \phi)=m t(\Delta x)+t(\delta x)=m \Delta l+t(\delta x)=m 2 r_{c}(\phi-\pi)+t(\delta x)
$$

since the time taken by the trajectory (which is always positive) equals the length of the trajectory $\left(v_{0}=1\right)$. What remains is to calculate the length of the trajectory for passing through the segment of length $\delta x$. This can be calculated easily, provided we know the angle $\varphi^{\prime}$ (see figure 5.12) made by the 


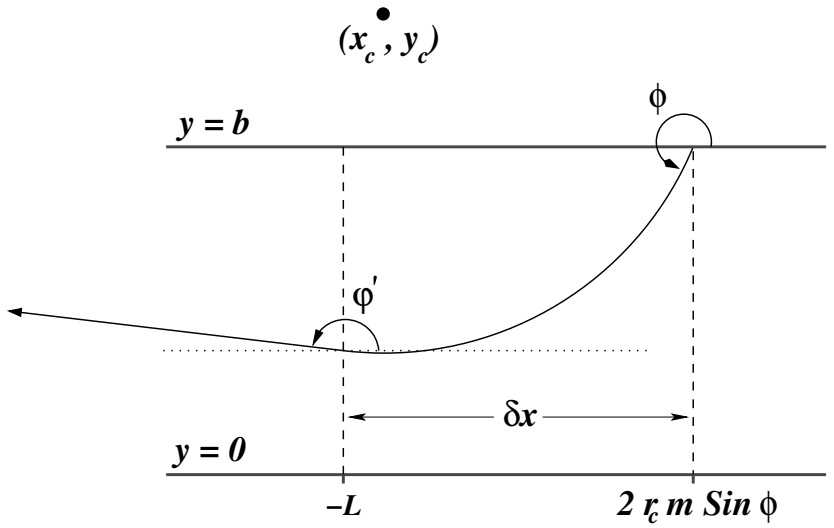

Figure 5.12: The center $\left(x_{c}, y_{c}\right)$ of the exit point and the beginning point for this last section of the trajectory of lateral distance $\delta x$ remains a constant. This provides a way for the calculation of the final angle $\varphi^{\prime}$ at the exit point.

trajectory at the exit point. For, if the length of this section of trajectory is $l$ then

$$
\varphi^{\prime}(l)=\phi-\frac{l}{r_{c}} \Rightarrow l=r_{c}\left(\phi-\varphi^{\prime}\right) .
$$

The final exit angle $\varphi^{\prime}$ can be calculated, if we note that the center for the exit point (at the angle $\phi^{\prime}$ ) and the initial point of this last section (of lateral distance $\delta x$ ) of the trajectory remains a constant. This provides,

$$
\begin{aligned}
x_{c}=x+r_{c} \sin \varphi^{\prime} & =2 m r_{c} \sin \phi+r_{c} \sin \phi=-L+r_{c} \sin \varphi^{\prime} \\
\Rightarrow \varphi^{\prime} & =\arcsin \left[(2 m+1) \sin \phi+\frac{L}{r_{c}}\right] .
\end{aligned}
$$

With this value of the exit angle $\varphi^{\prime}$ we can write the time taken by the last section (of lateral length $\delta x$ ) of the trajectory to be

$$
\begin{aligned}
t(\delta x) & =r_{c}\left(\phi-\varphi^{\prime}\right) \\
& =r_{c}\left(\phi-\arcsin \left[(2 m+1) \sin \phi+\frac{L}{r_{c}}\right]\right) .
\end{aligned}
$$

Therefore, the total time taken by a regular trajectory starting at a point $(0, b, \phi)$ and exiting a finite channel of length $L$ in the negative direction will be

$$
t(L, \phi)=2 m r_{c}(\phi-\pi)+r_{c}\left(\phi-\arcsin \left[(2 m+1) \sin \phi+\frac{L}{r_{c}}\right]\right),
$$




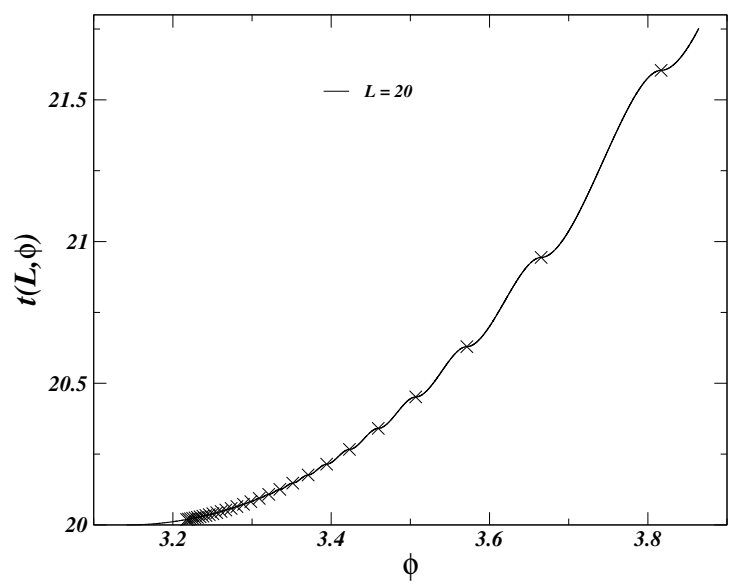

Figure 5.13: Variation of time $t(L, \phi)$ from equation (5.42) as a function of the angular coordinate $\phi$ for the trajectories starting at the point $(0, b)$. The points marked with a $\times$ show the stationary points of the curve where the partial derivative of time $t(L, \phi)$ with respect to the angular coordinate $\phi$ vanishes. Because of this vanishing derivative the classical probability diverges at these points, providing peaks in the delay time distribution.

where $m$ is given by

$$
m=\left[\frac{-L}{2 r_{c} \sin \phi}\right],
$$

with [...] denoting the integer part of the variable (...). In figure 5.13, we have plotted this time as a function of angular variation of $\phi$. We can observe several phenomena. Some of them are

- The time $t(L, \phi)$ increases as a function of the initial angle $\phi$. And since $|\sin \phi|$ increases as a function of $\phi \in[\pi: 3 \pi / 2]$, we conclude that the probability will also increase with the increase in time. This is observed in the delay time distributions (see figure 5.8) in having an average increase in the probability while increasing the value of time (apart from the occurrence of the divergences).

- There are some stationary points in the curve (marked by $\times$ ), for which the angular derivative of time vanishes. If we look into the delay time distribution (figure 5.8(a)), these points in time exactly corresponds to the points where the divergences occur.

By calculating the angles for which these stationary points appear, we find that at these points the length of the channel $L$ is exactly divisible by the lateral displacement $\Delta x$. In other words, we can fit exactly an integer number of arcs into the length of the channel. So, the peaks occur if the length of the channel is in commensuration with the lateral displacement $\Delta x$. These type of observations has also been seen in coherent electron focusing experiments [118]. Nevertheless, we need to understand why the delay probability becomes infinite at these points. If the probability for getting reflected at an angle $\phi$, for 
an electron at the point $(0, b)$, is $P(\phi)$, then the probability that a particle will exit at time $t(L, \phi)$ (for a channel of length $L$ ) will be proportional to $P(\phi)$. So, we can write the probability distribution $W_{R}(L, \tau)$ as

$$
W_{R}(L, \tau) \propto \int_{\phi} d \phi P(\phi) \delta[t(L, \phi)-\tau] .
$$

By the properties of the Dirac delta function, we can rewrite the above equation

$$
W_{R}(L, \tau) \propto \int_{\phi} d \phi P(\phi) \sum_{i} \frac{\delta\left(\phi-\phi_{i}\right)}{\left|f^{\prime}\left(\phi_{i}\right)\right|} .
$$

where $f(\phi)=t(L, \phi)-\tau$ and $\phi_{i}$ are the zeros of the function $f(\phi)$

$$
f\left(\varphi_{i}\right)=0 .
$$

Therefore, if $\tau$ is such that the derivative

$$
\frac{\partial t(L, \phi)}{\partial \phi}=0,
$$

then the integral in equation (5.44) diverges. This says that the divergences in the delay time distribution corresponds to the stationary points in the time curve. In other words divergences in the delay time distribution of the regular trajectories occur if the lateral length $\Delta x$ is in commensuration with the finite length of the channel. This also explains that if the length of the channel increases there will be larger number of peaks visible in the distribution as more and more nodes appear for which the total length $L$ is in commensuration with the lateral length $\Delta x$ of the trajectory.

\subsubsection{General set of trajectories}

Unlike the special set of trajectories, where the electrons only enter at $(0, b, \phi)$, the electrons traveling through the general set of trajectories enter as in case of an electronic setup (of the finite channel as a wire). The electrons enter from the right hand side of the channel at any point in the regular phase space region and exit at any point depending on their initial point. One such trajectory is shown in figure 5.14. Once the trajectory hits the boundary $y=b$ the dynamics can be described as in the case of special set of trajectories. Nevertheless we need to take into account the initial section of trajectory joining the point $(0, y)$ to the boundary $y=b$. Additionally we can extend this trajectory to a previous point inside the reservoir $(x, b, \phi)$, such that the dynamics does not change, (see figure 5.14) and subtract the additional length we have extended to the reservoir. We will follow the later approach. We already have calculated the time taken by a trajectory entering at $(0, b, \phi)$ to exit a finite channel of length $L$. In extending the trajectory into the reservoir we effectively have extended the channel length by an amount $x$. Therefore, the total time $t(L, \varphi)$ taken by the trajectory from the point $(0, y, \varphi)$ inside 


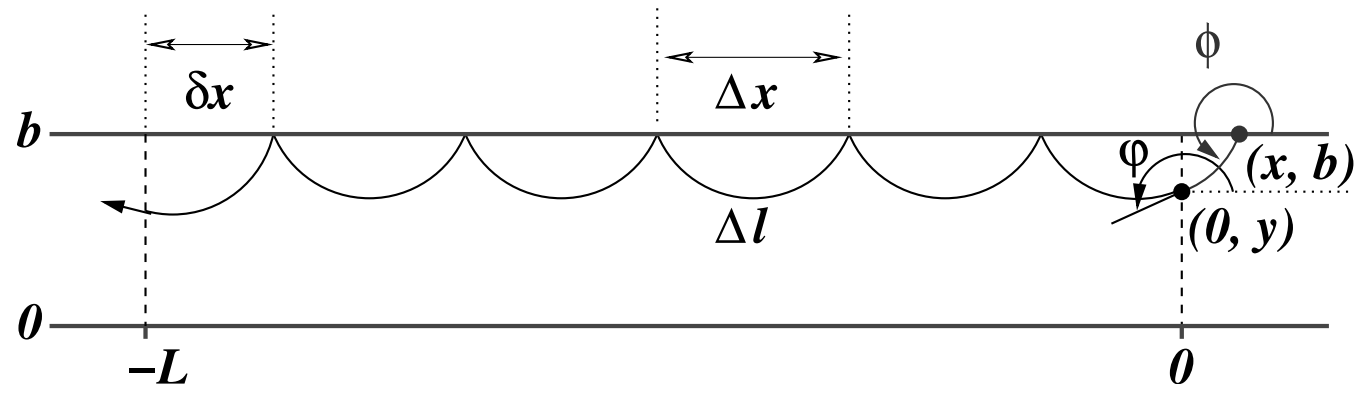

Figure 5.14: Any general regular electron entering the channel at $(0, y, \varphi)$ can be mapped to a point inside the reservoir $(x, b, \phi)$. Because of this mapping we extend the horizontal length of the trajectory by an amount $x$ into the reservoir. This simple construction helps to describe the dynamics in terms of the special set of trajectories (which are much more simpler). Nevertheless, we need to take care of this extra lateral displacement of $x$.

the reservoir to exit the channel will be

$$
t(L, y, \varphi)+t(x)=2 m r_{c}(\phi-\pi)+r_{c}\left(\phi-\arcsin \left[(2 m+1) \sin \phi+\frac{L+x}{r}\right]\right) .
$$

where $t(x)$ is the additional time we have added in extending the trajectory into the reservoir $(x, b, \phi)$ and $m$ is given by

$$
m=\left[\frac{-(L+x)}{2 r_{c} \sin \phi}\right] .
$$

To calculate the relation between $\phi$ and $\varphi$; and $x, t(x)$, we need to consider that the starting point of the trajectory $(0, y, \phi)$ and the extended point $(x, b, \phi)$ share a common center of curvature $\left(x_{c}, y_{c}\right)$ and the length of the trajectory is related to the angles $\varphi$ and $\phi$ by the relation

$$
l=r_{c}(\phi-\varphi)=t(x) .
$$

From the common value of $y_{c}$, we obtain

$$
y_{c}=y-r_{c} \cos \varphi=b-r_{c} \cos \phi \text {. }
$$

It provides the value of $\phi$ as

$$
\phi=\arccos \left(\frac{b-y}{r_{c}}+\cos \phi\right) .
$$

Now to find out the value of $x$, we use the other common point $x_{c}$. This provides

$$
x_{c}=0+r_{c} \sin \varphi=x+r_{c} \sin \phi \Rightarrow x=r_{c}(\sin \varphi-\sin \phi) .
$$




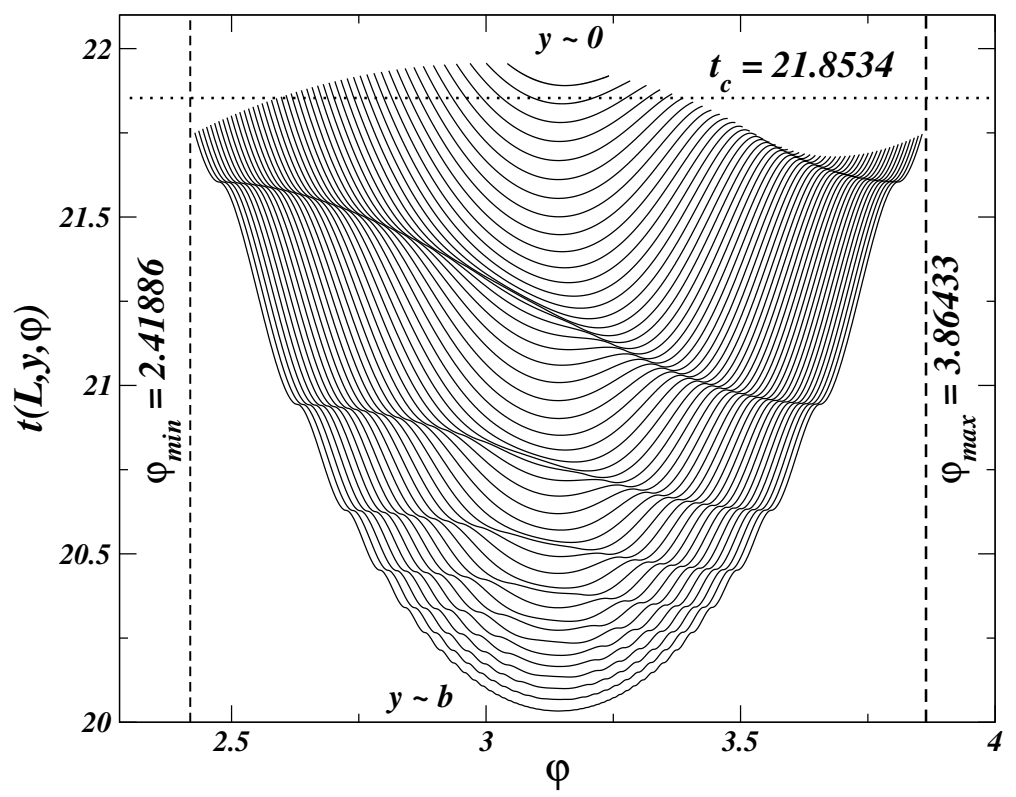

Figure 5.15: The variation of time $t(L, y, \varphi)$ as in equation (5.54) as a function $\varphi$ for various values of y. $y \simeq b$ corresponds to the outer curves and then it decreases to zero towards the inside. The critical average time $t_{c}$ is plotted with a dotted line and $\varphi_{\min }, \varphi_{\max }$ are the outer boundaries for the allowed values of $\varphi$ when $y \rightarrow b$. The different curves corresponding to different values of $y$ do not intersect each other. They initially decrease as $\varphi$ increases to $\pi$ and then increase with increasing the value of $\varphi$.

Substituting all of these values we obtain the final result in terms of $y$ and $\varphi$ as

$$
\begin{gathered}
t(L, y, \varphi)=(2 m+1) r_{c} \arccos \left(\frac{y-b}{r}-\cos \varphi\right) \\
+r_{c} \arcsin \left\{\frac{L}{r_{c}}+r_{c} \sin \varphi-2 m \sin \left[\arccos \left(\frac{y-b}{r_{c}}-\cos \varphi\right)\right]\right\} \\
+r_{c}\left[\varphi-\pi-\arccos \left(\frac{y-b}{r_{c}}-\cos \varphi\right)\right]
\end{gathered}
$$

The results are shown in figure 5.15. From this figure, we note that :

- The curves corresponding to different values of $y$ do not intersect with each other.

- For some values of angles $\varphi$ the angular derivative of $t(L, y, \varphi)$ vanishes i.e.,

$$
\left.\frac{\partial t(L, y, \varphi)}{\partial \varphi}\right|_{y}=0 .
$$

These points form a dense structure of strictures (curves) running from left hand side of the plot 
to the right hand side of the plot.

We have discussed (in section 5.5.2) that the points where the angular derivative of time vanishes correspond to a peak in the probability density. Now the question arises if this is really the cause of the peaks in the probability density. At first glance it is clear that indeed this is not so. Because the strictures look rather continuous as a function of time. Once they vanish at the right hand side they reappear on the left hand side of the plot. However the peaks occur only at the right hand point of the strictures (where they vanish). To complete this picture we add the time obtained for the special set of trajectories and plot the points where the peaks occur. This resulting curve along with the distribution is shown in figure 5.16. We observe, indeed the peak positions correspond to the times where the strictures vanish

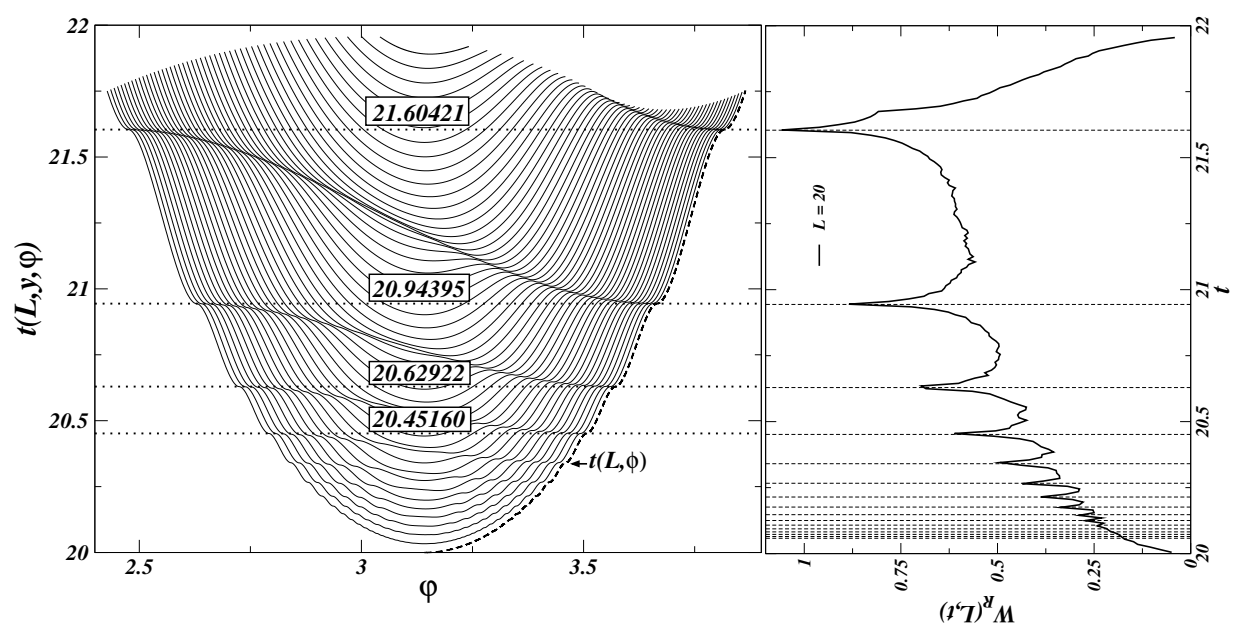

Figure 5.16: The peaks in the distribution occur only when the derivative of $t(L, \phi)$ vanishes. These points corresponds to the end points of the strictures (thick black lines) on the higher values of $\varphi$ and reappearance of them on the lower value of $\varphi$. Moreover, the time derivative of the probability will follow a pattern as like these strictures. It is not clear if there is some correspondence between these strictures and the derivative of the probability distribution.

(towards the higher value of angular coordinate $\varphi$ ) and reappear (towards the lower value of angular coordinate $\varphi$ ). In other words they corresponds to the points where the angular derivative of $t(L, \phi)$ vanishes which specifies only a small set of points for which the angular derivative of $t(L, y, \varphi)$ vanish at constant $y$. Therefore, the trajectories starting at $(0, b, \phi)$ deserve a special place in the probability distribution. There is something more as well. The derivative of the delay time distribution will follow a pattern as like the strictures in the time plot. Nonetheless, it is not immediately clear if there is one to one correspondence between the derivative of the probability distribution w.r.t. time and the strictures in the time plot. Quantitatively we can think of the probability distribution as

$$
W_{R}(L, \tau) \propto \int_{y} d y \int_{\varphi} P(\varphi) \delta(t(L, y, \varphi)-\tau) d \varphi
$$


where $P(\varphi)=(1 / 2) \cos \varphi$ is the probability density of an electron to enter into the channel from the reservoir at an angle $\varphi$. Since, the delta function involves both the values $y$ and $\varphi$, the integration will involve derivatives of $t(L, y, \varphi)$ w.r.t. $y$ as well as $\varphi$. Therefore, we suspect that both the derivatives w.r.t. $y$ and w.r.t. $\varphi$ vanish at these points of the curve where there is a discontinuity in the strictures. Where as on the strictures inside the curve i.e., for times not corresponding to the peaks at least one of the derivative of $t(L, y, \varphi)$ remains finite, providing a finite value for the delay time probability. This can

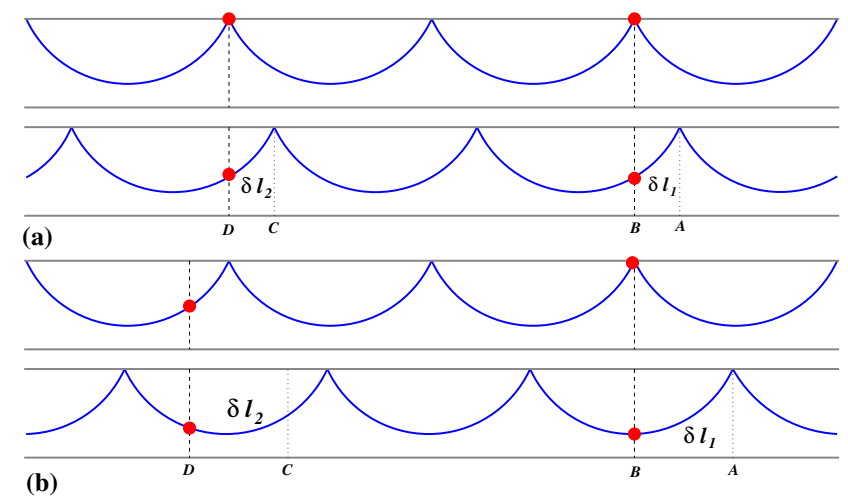

Figure 5.17: (a) If the lateral displacement $\Delta x$ is in commensuration with the length of the finite channel then all the points on the trajectory contribute to the same delay time (i.e., $\delta l_{1}-\delta l_{2}=0$ ), whereas if the trajectory is not in commensuration with the length of the channel, as in (b), then the points on the trajectory contribute to probabilities at different times $\left(\delta l_{1}-\delta l_{2} \neq 0\right)$. Therefore, in the former case we expect a peak in the distribution whereas in the latter case we may not expect one.

be understood in the following way as well. Think of a trajectory which is in commensuration with the length of the channel (see figure 5.17(a)). Then all the points on the curve will contribute to the same time i.e., if we move the trajectory either right hand side or left hand side then the value of $y$ as well as $\varphi$ will change but the time will remain the same. As a result the probability will diverge. Whereas if we change the trajectory such that it is not in commensurability with the length of the channel (see figure 5.17 (b)) then on moving over the same trajectory will result in having a different time at the exit. Therefore, they will not contribute to the peaks in the distribution.

So qualitatively we are able to explain the occurrence of peaks as constructively contributing to the same time for different trajectories. Mathematically we argue that these peaks occur only if both the spatial $y$ and the angular $\varphi$ derivative of the time function $t(L, y, \varphi)$ vanish. Nevertheless it remains to prove the occurrence of the peaks on the basis of the integral (5.56). In the following section we detail out the mathematics involving this prove. 


\subsubsection{Occurrence of peaks in the probability distribution}

For our purpose of the proof we assume that the upper wall of the channel is disordered and the lower wall support specular reflections. This does not change the dynamics of the distribution but interchanges the signs of the velocities of the regular and chaotic trajectories. Then the distribution function $W(L, \tau)$, which is a function of $t(L, y, \varphi)$, and is distributed according to the measure

$$
d \mu_{\perp, \mathrm{reg}}=\frac{1}{2} d y d \varphi \cos \varphi \Theta\left(y-r_{c}[1+\cos \varphi]\right)
$$

as

$$
W(L, \tau)=\frac{1}{\mu_{\perp, \text { reg }}} \int d \mu_{\perp, \text { reg }} \delta(T(L, y, \varphi)-\tau)
$$

can be written by mapping the entrance point $(y, \varphi)$ to a point on the boundary $(\xi, \phi)(\xi$ is the distance of the first reflection at the clean wall from the entrance into the system and $\phi$ is the base angle at this reflection). Then the phase space measure in equation (5.57) can be written as

$$
d \mu_{\perp, \mathrm{reg}}=\frac{1}{2} d \phi \sin \varphi \Theta\left(\cos \phi-1+\frac{b}{r_{c}}\right) 2 d \xi \Theta\left(\frac{\Delta x(\phi)}{2}-|\xi|\right) .
$$

The first $\Theta$ function restricts the variable $\phi$ to the regular components only, providing

$$
0 \leqslant \phi \leqslant \phi_{\max }
$$

where

$$
\phi_{\max }=\arccos \left(1-\frac{b}{r_{c}}\right),
$$

and the second $\Theta$ function just says that all the relative locations between the entrance to the channel $x=0$ and the base point are equivalent. $\Delta x(\phi)=2 r_{c} \sin \phi$ is the base length of an arc with base angle $\phi \geq 0$. The longitudinal dislocation of $\xi$ of the trajectory we choose such that

$$
-\frac{\Delta x(\phi)}{2} \leqslant \xi \leqslant \frac{\Delta x(\phi)}{2} .
$$

By analogy we have again

$$
W(L, \tau)=\frac{1}{\mu_{\perp, \text { reg }}} \int d \phi \sin \varphi \Theta\left(\cos \phi-1+\frac{b}{r_{c}}\right) d \xi \Theta\left(\frac{\Delta x(\phi)}{2}-|\xi|\right) \delta(t(L, \phi, \xi)-\tau) .
$$

As already mentioned, by the properties of the Dirac delta function, the evaluation of this integral involves the derivatives of the function $t(L, \phi, \xi)-\tau$. Therefore, it is sufficient to show that the points obtained during the analysis of the special set of trajectories are only the points where the divergences occur. For this we note that, the time taken by a typical trajectory (as shown in figure 5.18) entering the 


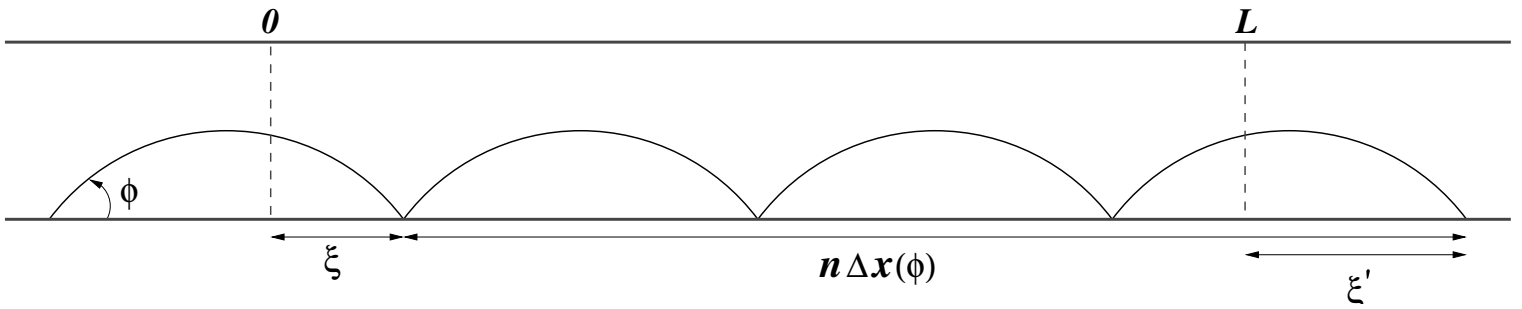

Figure 5.18: The total time taken by the trajectory can be calculated using the addition of all the times taken by the electron for each individual sections of the trajectory before it comes out of the channel.

channel at $(x=0, y, \varphi)$ is

$$
t(L, y, \varphi)=n \Delta l(\phi)-s(\phi, n \Delta x(\phi)-L+\xi)+s(\phi, \xi),
$$

where $s$ represents the time taken by the electron before (after) the initial reflection (final exit) from inside the channel, and $n$ is given by

$$
n=\left[\frac{L}{\Delta x(\phi)}+\frac{1}{2}\right] .
$$

As $\Delta l(\phi)=2 r_{c} \phi$ is the time taken by the electron between two consecutive reflections from the lower wall (this is equal to the length of the trajectory between the reflections) inside the channel, we can write the total time as

$$
t(L, \phi, \xi)=2 r_{c} n \phi-s\left(\phi, 2 r_{c} n \sin \phi-L+\xi\right)+s(\phi, \xi) .
$$

Using the base angle $\phi$ and the entrance angle $\varphi$ we can calculate the horizontal dislocation $\xi$ and the arc length $s$ as

$$
\begin{aligned}
2 \xi=\Delta x(\phi)-\Delta x(\varphi) & =2 r_{c}(\sin \phi-\sin \varphi), \\
2 s=\Delta l(\phi)-\Delta l(\varphi) & =2 r_{c}(\phi-\varphi) .
\end{aligned}
$$

Using the above two equations we can write

$$
\begin{aligned}
s(\phi, \xi) & =r_{c}(\phi-\varphi) \\
& =r_{c}\left[\phi-\arcsin \left(\sin \phi-\frac{\xi}{r_{c}}\right)\right] .
\end{aligned}
$$

In particular we note that

$$
s(\phi, 0)=0 \quad \text { and } \quad s(\phi, \Delta x(\phi) / 2)=r_{c} \phi,
$$


and we extend this by the definition

$$
\begin{aligned}
s(\phi,-\xi) & =-s(\phi, \xi) \\
s(\phi, \xi+\Delta x(\phi)) & =s(\phi, \xi) .
\end{aligned}
$$

Using these definitions we note that for $L=n \Delta x(\phi)$, the delay time $t(L, y, \phi)=n \Delta l(\phi)$ does not depend on the dislocation $\xi$. Moreover these values of $\phi=\phi_{n}$ are stationary positions of the delay time for zero dislocation $(\xi=0)$ since

$$
\begin{aligned}
\left.\frac{d}{d \phi} t(L, \phi, 0)\right|_{\phi=\phi_{n}} & =\frac{d}{d \phi}\left[2 r_{c} n \phi-s\left(\phi, 2 r_{c} n \sin \phi-L\right)+s(\phi, 0)\right]_{\phi=\phi_{n}} \\
& =2 r_{c} n-\left.\frac{\partial s\left(\phi, 2 r_{c} n \sin \phi-L\right)}{\partial \phi}\right|_{\phi=\phi_{n}}-\left.\frac{\partial s\left(\phi, 2 r_{c} n \sin \phi-L\right)}{\partial \xi^{\prime}}\right|_{\phi=\phi_{n}} \times 2 r_{c} n \cos \phi_{n}
\end{aligned}
$$

Now from equation (5.69) we note that

$$
\frac{\partial s\left(\phi, \xi^{\prime}\right)}{\partial \phi}=r_{c}\left(1-\frac{\cos \phi}{\sqrt{1-\left(\frac{\xi^{\prime}}{r_{c}}-\sin \phi\right)^{2}}}\right)
$$

and

$$
\frac{\partial s\left(\phi, \xi^{\prime}\right)}{\partial \xi^{\prime}}=\frac{1}{\sqrt{1-\left(\frac{\xi^{\prime}}{r_{c}}-\sin \phi\right)^{2}}}
$$

which for $\phi=\phi_{n}$ (or $\xi^{\prime}=2 r_{c} n \sin \phi-L=0$ ) becomes

$$
\frac{\partial s(\phi, 0)}{\partial \phi}=0 \quad \text { and } \quad \frac{\partial s(\phi, 0)}{\partial \xi^{\prime}}=\sec \phi_{n}
$$

Substitution of these values into equation (5.72) yields

$$
\begin{aligned}
\left.\frac{d}{d \phi} t(L, \phi, 0)\right|_{\phi=\phi_{n}} & =2 r_{c} n-0-\sec \phi_{n} \times 2 r_{c} n \cos \phi_{n} \\
& =0 .
\end{aligned}
$$

However if $\xi=0$ but $\phi \neq \phi_{n}$ then the derivatives in the equations (5.73) and (5.74) still contributes finite values. Therefore, we proved that the peaks occur only at those points, such that the longitudinal displacement $\Delta x(\phi)$ is in commensuration with the length of the channel $L$, set by the special set of trajectories only. 


\subsection{Summary and Discussion}

In this chapter we extended the discussion of the disordered billiard channel, studied in the previous chapters, into studying the classical time delay of trajectories. We show that the chaotic time delay follow the directed behavior of the electrons by having a peak at the point of its average position and showed that for larger time scales and small system lengths, the distribution would follow a decay of exponential nature. This is different from what people have observed in disordered systems [96, 97, 98, 99, 100, 101]. And unlike the power law behavior [98, 99, 100, 104, 105, 109, 114] we obtain an exponential decay for shorter system sizes. Also we are able to show that the reflection time delay will follow a combination of exponential and power law behavior, and is independent of the length of the channel for large system lengths. What is interesting in the system is the electrons move in different ways while transmitting from the left hand side of the channel than while transmitting from right hand side of the channel. In the former case all the trajectories are chaotic having an average non zero velocity. While in the later case we have mostly regular trajectories transmitting through the channel. It is interesting to note that; even though the transmission in both directions are independent of direction, they show different behaviors in terms of their time delays. For the chaotic trajectories directing along the channel with a positive velocity provide only a single peak characterizing the average velocity of the electron, while the regular trajectories provide peaks in the distribution. Qualitatively, we are able to show that the peaks are divergences in the probability distribution. Nevertheless, it still remains to evaluate the un-formidable looking integral (5.56) for any quantitative understanding of the entire dynamics. 


\section{Chapter 6}

\section{Summary and Outlook}

"There is no end, but at every end there is always a new beginning ..."

We reported our study of directed chaotic motion in quasi-one-dimensional billiard chains in the presence of a moderate magnetic field, s.t. the corresponding cyclotron radius $r_{c}>b$ ( $b$ is the width of the channel). It leads to a simple phase space containing a mixture of two sets. One consisting of regular trajectories, which specularly get reflected from one of the clean walls of the channel and the other a chaotic trajectory due of heavy scattering (a cause of disorder or periodically placed scatterers). At thermal equilibrium the net transport arising from these two sets of trajectories cancel each other. This provided a way to calculate the average chaotic transport velocity using the underlying phase space structure. We also are able to understand qualitatively and quantitatively the aspects of transmission in the case of a finite disordered wire. In short we show that the transport is independent of direction in absence of any bias, and approaches a constant value if the length of the system $L \rightarrow \infty$. Using a novel combination of Fokker-Planck Equation (FPE) for biased diffusion and a Biased Persistent Random Walk (BPRW) we show that the decrease in transmission to this constant value is exponential. Also we found that there is an underlying probabilistic equation under the BPRW model, which can be expressed as an equivalent FPE in the infinite limit of $x \rightarrow \infty, t \rightarrow \infty$. Within this limit, we show that the Peclet length scales of the FPE and the BPRW can be equal for low bias but in general they are not equal. Nonetheless it remains to be seen, if we can apply the microscopic picture of the BPRW (involving the calculation of reflection probabilities $r$ and $r^{\prime}$ ) independent of the FPE to calculate the transmission probability associated with the system. We also studied the time delay properties and show that the directed behavior of the chaotic trajectories is retained for the transmission of chaotic trajectories. In contrast the reflection delay times of the chaotic trajectories remains independent of the system length for large values of system lengths. It is known that disordered wires show localization effects and universal conductance fluctuations. So, it will be interesting to do the quantum version of this model to look for such effects. Moreover the transmission time delays of the chaotic trajectories have been calculated using the FPE only. So, what will happen to the time delay probabilities if we 
replace the FPE by the BPRW ? 
Appendices 



\section{Appendix A}

\section{Length of a section on the trajectory}

To calculate the length of a section of a trajectory in terms of its inclination angle $\phi$ we note that when $\phi^{\prime}=0$, see figure A.1 a), the angle made by the end points of the section of the trajectory ( $A$ and $B$ ) at the center of the current arc's circle is $2 \phi$. This can be calculated using the knowledge that, the tangent at a point on the circle is perpendicular to the radial vector of the circle at that point. Therefore, the angle $\angle B A C=\pi / 2-\phi$. Also the triangle $\triangle A B C$ is an isosceles triangle with the sides $A C$ and $B C$ of equal length, the length being equal to cyclotron radius $r_{c}$. From the properties of an isosceles triangle we have the $\angle A B C=\angle B A C=\pi / 2-\phi$. We also know from the properties of the triangles that the sum of all the interior angles is equal to $\pi$ (or 180 degrees). Therefore, the end points of the current arc will make an angle

$$
\angle A C B=\pi-(\angle A B C+\angle B A C)=2 \phi
$$

(a)

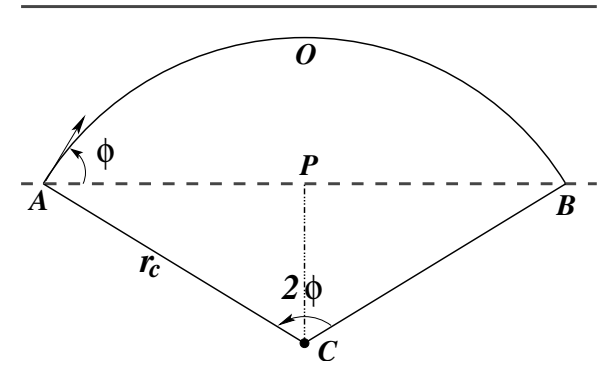

(b)

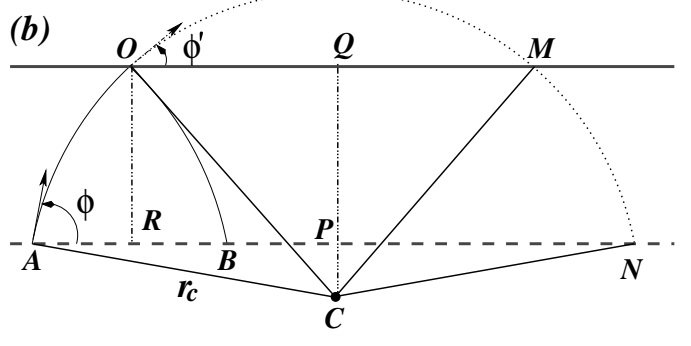

Figure A.1: Section of a trajectory $(A O B)$ between two successive reflections from the lower boundary (points $A$ and $B$ ) is shown here with an intermediate point $O$ for $(a) \phi^{\prime}=0$ and (b) $\phi^{\prime} \neq 0 . \phi$ is the inclination angle of the arc with the lower boundary and $\phi^{\prime}$ is the inclination angle of the section of the arc at the upper wall of the channel. 
at the center of the current cyclotron circle. Hence, the length of the current section of the trajectory will be

$$
\Delta l(\phi)=2 r_{c} \phi
$$

To calculate the horizontal distance traveled during this section of the trajectory, we could drop a perpendicular from the center of the current cyclotron orbit to the line $A B$. This perpendicular line will divide the line $A B$ into two sections of equal length at some point (denoted by $P$ ). Since the angle $\angle B A C=\angle P A C=\pi / 2-\phi$, by triangle law we can write the length of section $A P$ as $|\overline{A P}|=$ $r_{c} \cos (\angle P A C)=r_{c} \sin \phi$, providing the total horizontal length of this section of trajectory $|\overline{A B}|=$ $2|\overline{A P}|=2 r_{c} \sin \phi$.

On the other hand when $\phi^{\prime} \neq 0$ (figure A.1 $(b)$ ) we can extend the trajectory, as if the upper wall is not there, to another point $N$ on the lower wall. Then as discussed above we will have the values of the angles $\angle A C N=2 \phi$ and $\angle O C M=2 \phi^{\prime}$. By symmetry we can say that the section of the arc $O B$ and the arc $M N$ are of equal length. Therefore the length of the section of the trajectory traveled by the electron will be equal to $|\overparen{\mathrm{AO}}|+|\overparen{\mathrm{OB}}|=|\overparen{\mathrm{AO}}|+|\overparen{\mathrm{MN}}=| \mathrm{AOMN}|-| \overparen{\mathrm{OM}} \mid$. Since the angle $\angle A C N=2 \phi$ therefore, $|\mathrm{AOMN}|=2 r_{c} \phi$ and since $\angle O C M=2 \phi^{\prime}$ we will have $|\overparen{\mathrm{OM}}|=2 r_{c} \phi^{\prime}$. This will provide the length of the section of the trajectory when $\phi^{\prime} \neq 0$ to be

$$
\Delta l(\phi)=2 r_{c}\left(\phi-\phi^{\prime}\right) .
$$

In order to calculate the horizontal distance traveled by this section of trajectory i.e., $|\overline{A B}|$ we drop another perpendicular from the reflection point of the trajectory at the upper wall (shown with the point $O$ in figure A.1 $(b)$ ) to the lower boundary. Then the point at the lower boundary $P$ divides the segment $A B$ into two equal halfs. Now from construction; $|\overline{R P}|=|\overline{O Q}|=r_{c} \sin \phi^{\prime}$. Therefore, $|\overline{A R}|=|\overline{A P}|$ $|\overline{R P}|=r_{c}\left(\sin \phi-\sin \phi^{\prime}\right)$. Thus providing the value of horizontal length of this section of trajectory when $\phi^{\prime} \neq 0$ to be $|\overline{A B}|=2|\overline{A R}|=2 r_{c}\left(\sin \phi-\sin \phi^{\prime}\right)$.

What remained is to calculate the value of $\phi^{\prime}$ in terms of $\phi$. In order to do this we note that $|\overline{C Q}|=r_{c} \sin (\angle Q O C)=r_{c} \cos \phi^{\prime}$. Similarly $|\overline{P C}|=r_{c} \sin (\angle P A C)=r_{c} \cos \phi$. Since $|\overline{P Q}|=b$ and $|\overline{C Q}|$ $=|\overline{C P}|+|\overline{P Q}|$ we find

$$
r_{c} \cos \phi^{\prime}=r_{c} \cos \phi+b .
$$

This provides the result for $\phi^{\prime}$ as in equation (3.8) to be

$$
\phi^{\prime}=\arccos \left(\cos \phi+\frac{b}{r_{c}}\right) .
$$

This equation will be valid when the upper most point on the arc remains above the upper boundary i.e., when

$$
y_{c}+r_{c} \geqslant b \quad \text { or } \quad-r_{c} \cos \phi+r_{c} \geqslant b \Rightarrow \cos \phi \leqslant 1-\frac{b}{r_{c}} .
$$


When $\phi^{\prime}=0$, we have $\sin \phi^{\prime}=0$. So, we can extend all of these equations into a single equation and write

$$
\begin{aligned}
\Delta x(\phi) & =2 r_{c}\left(\sin \phi-\sin \phi^{\prime}\right) \\
\text { and } \Delta l(\phi) & =2 r_{c}\left(\phi-\phi^{\prime}\right) .
\end{aligned}
$$

where $\phi^{\prime}$ is given by

$$
\phi^{\prime}=\left\{\begin{array}{ll}
\arccos \left(\cos \phi+\frac{b}{r_{c}}\right) & \left(\cos \phi \leqslant 1-\frac{b}{r_{c}}\right) \\
0 & \left(\cos \phi>1-\frac{b}{r_{c}}\right)
\end{array},\right.
$$

in conformity with equations (3.6) (3.7) and (3.8). 



\section{Appendix B}

\section{Jacobian of a transformation}

Given a set of equations $\vec{y}=\left\{y_{i}: i=1,2, \ldots, n\right\}$ in $n$ variables $\vec{x}=\left\{x_{i}: i=1,2, \ldots, n\right\}$

$$
\begin{aligned}
y_{1} & =f_{1}\left(x_{1}, x_{2}, \ldots, x_{n}\right) \\
y_{2} & =f_{2}\left(x_{1}, x_{2}, \ldots, x_{n}\right) \\
& \vdots \\
y_{n} & =f_{n}\left(x_{1}, x_{2}, \ldots, x_{n}\right),
\end{aligned}
$$

which can be written in the form

$$
\vec{y}=\left[\begin{array}{l}
f_{1}(\vec{x}) \\
f_{2}(\vec{x}) \\
\vdots \\
f_{n}(\vec{x})
\end{array}\right]
$$

the Jacobian Matrix of this transformation is defined by

$$
J\left(x_{1}, x_{2}, \ldots, x_{n}\right)=\left[\begin{array}{lll}
\frac{\partial y_{1}}{\partial x_{1}} & \cdots & \frac{\partial y_{1}}{\partial x_{n}} \\
\vdots & \ddots & \vdots \\
\frac{\partial y_{n}}{\partial x_{1}} & \cdots & \frac{\partial y_{n}}{\partial x_{n}}
\end{array}\right]
$$

The determinant of this matrix, also called the Jacobian, is denoted by

$$
J=\left|\frac{\partial\left(y_{1}, y_{2}, \ldots, y_{n}\right)}{\partial\left(x_{1}, x_{2}, \ldots, x_{n}\right)}\right|
$$


Taking the differential of equations (B.2), we find

$$
\left[\begin{array}{l}
d y_{1} \\
d y_{2} \\
\vdots \\
d y_{n}
\end{array}\right]=\left[\begin{array}{lll}
\frac{\partial y_{1}}{\partial x_{1}} & \cdots & \frac{\partial y_{1}}{\partial x_{n}} \\
\vdots & \ddots & \vdots \\
\frac{\partial y_{n}}{\partial x_{1}} & \cdots & \frac{\partial y_{n}}{\partial x_{n}}
\end{array}\right]\left[\begin{array}{l}
d x_{1} \\
d x_{2} \\
\vdots \\
d x_{n}
\end{array}\right]
$$

Thus, the Jacobian $J$ provides the ratios of n-dimensional volumes in $y$ and $x$

$$
d y_{1} d y_{2} \ldots d y_{n}=\left|\frac{\partial\left(y_{1}, y_{2}, \ldots, y_{n}\right)}{\partial\left(x_{1}, x_{2}, \ldots, x_{n}\right)}\right| d x_{1} d x_{2} \ldots d x_{n} .
$$

For the transformation in equations $3.97-3.11$

$$
\begin{aligned}
x & =x \\
\varphi(\phi, l) & = \begin{cases}\phi-\frac{l}{r_{c}} & l \leqslant \frac{\Delta l(\phi)}{2} \\
-\varphi(\phi, \Delta l-l) & l>\frac{\Delta l(\phi)}{2}\end{cases} \\
y(\phi, l) & =r_{c} \cos \varphi(\phi, l)-r_{c} \cos \phi,
\end{aligned}
$$

the Jacobian matrix will be

$$
\left[\begin{array}{lll}
\frac{\partial x}{\partial x} & \frac{\partial x}{\partial l} & \frac{\partial x}{\partial \phi} \\
\frac{\partial y}{\partial x} & \frac{\partial y}{\partial l} & \frac{\partial y}{\partial \phi} \\
\frac{\partial \varphi}{\partial x} & \frac{\partial \varphi}{\partial l} & \frac{\partial \varphi}{\partial \phi}
\end{array}\right]=\left\{\begin{array}{ccc}
{\left[\begin{array}{ccc}
1 & 0 & 0 \\
0 & \sin \left(\phi-\frac{l}{r_{c}}\right) & -r_{c} \sin \left(\phi-\frac{l}{r_{c}}\right)+r \sin \phi \\
0 & -\frac{1}{r_{c}} & 1
\end{array}\right] \quad l \leqslant \frac{\Delta l(\phi)}{2}} \\
{\left[\begin{array}{ccc}
1 & 0 & 0 \\
0 & \sin \left(\frac{\Delta l-l}{r_{c}}-\phi\right) & r_{c} \sin \left(\frac{\Delta l-l}{r_{c}}-\phi\right)+r_{c} \sin \phi \\
0 & -\frac{1}{r_{c}} & -1
\end{array}\right] \quad l>\frac{\Delta l(\phi)}{2}}
\end{array} .\right.
$$

The determinant of both the matrices is then

$$
J=\sin \phi=2 P(\phi),
$$

since the normalized probability of scattering at the lower wall at an inclination angle $\phi$ is (refer to equation (3.1)

$$
P(\phi)=\frac{1}{2} \sin \phi \quad 0 \leqslant \phi \leqslant \pi .
$$




\section{Appendix C}

\section{Calculation of $\langle\Delta x\rangle_{\phi}$ and $\langle\Delta l\rangle_{\phi}$}

The value of $\Delta x$ and $\Delta l$ are given by

$$
\Delta x(\phi)=2 r_{c}\left(\sin \phi-\sin \phi^{\prime}\right), \quad \delta l(\phi)=2 r_{c}\left(\phi-\phi^{\prime}\right),
$$

where $\phi^{\prime}$ is given in equation (3.8). Therefore, with $P(\phi)=(1 / 2) \sin \phi$ we can calculate

$$
\begin{aligned}
\langle\Delta x\rangle_{\phi} & =r_{c} \int_{0}^{\pi} d \phi \sin \phi\left(\sin \phi-\sin \phi^{\prime}\right)=-r_{c} \int_{0}^{\pi} d(\cos \phi)\left(\sqrt{1-\cos ^{2} \phi}-\sqrt{1-\cos ^{2} \phi^{\prime}}\right) \\
& =r_{c} \int_{-1}^{+1} d c \sqrt{1-c^{2}}-r_{c} \int_{-1}^{1-b / r_{c}} d c \sqrt{1-\left(c+\frac{b}{r}\right)^{2}} \quad(\text { where } c=\cos \phi) \\
& =\frac{r_{c}}{2}\left[c \sqrt{1-c^{2}}+\arcsin c\right]_{-1}^{1}-\frac{r_{c}}{2}\left[\left(c+\frac{b}{r_{c}}\right) \sqrt{1-\left(c+\frac{b}{r_{c}}\right)^{2}}+\arcsin \left(c+\frac{b}{r_{c}}\right)\right]_{-1}^{1-b / r_{c}} \\
& =\frac{\pi}{2} r_{c}-\frac{r_{c}}{2}\left[\frac{\pi}{2}+\left(1-\frac{b}{r_{c}}\right) \sqrt{\frac{b}{r_{c}}\left(2-\frac{b}{r_{c}}\right)}-\arcsin \left(\frac{b}{r_{c}}-1\right)\right] \\
& =\frac{\pi}{2} r_{c}-\frac{r_{c}}{2}\left[\frac{\pi}{2}+\cos \theta \sqrt{(1-\cos \theta)(1+\cos \theta)}+\arcsin (\cos \theta)\right] \quad\left(\cos \theta=1-\frac{\mathrm{b}}{\mathrm{r}_{\mathrm{c}}}\right) \\
& =\frac{r_{c}}{2}\left[\left(\frac{\pi}{2}-\arcsin (\cos \theta)\right)-\cos \theta \sin \theta\right] \\
& =\frac{r_{c}}{2}[\arccos (\cos \theta)-\cos \theta \sin \theta] \\
& =\frac{r_{c}}{2}(\theta-\sin \theta \cos \theta),
\end{aligned}
$$


142

Chapter C. Calculation of $\langle\Delta x\rangle_{\phi}$ and $\langle\Delta l\rangle_{\phi}$

and

$$
\begin{aligned}
\langle\Delta l\rangle_{\phi} & =r_{c} \int_{0}^{\pi} d \phi \sin \phi\left(\phi-\phi^{\prime}\right) \\
& =-r_{c} \int_{0}^{\pi} d(\cos \phi)\left[\arccos (\cos \phi)-\arccos \left(\cos \phi+\frac{b}{r_{c}}\right)\right] \\
& =r_{c} \int_{-1}^{+1} d c \arccos (c)-r_{c} \int_{-1}^{1-b / r_{c}} d c \arccos \left(c+\frac{b}{r_{c}}\right) \quad(\text { where } c=\cos \phi) \\
& =r_{c}\left[c \arccos (c)-\sqrt{1-c^{2}}\right]_{-1}^{1}-r_{c}\left[\frac{\pi}{2} c-\left(c+\frac{b}{r_{c}}\right) \arcsin \left(c+\frac{b}{r_{c}}\right)-\sqrt{1-\left(c+\frac{b}{r_{c}}\right)^{2}}\right]_{-1}^{1-b / r_{c}} \\
& \left.=\pi r_{c}-r_{c}\left\{\left(1-\frac{b}{r_{c}}\right)\left[\frac{\pi}{2}+\arcsin \left(1-\frac{b}{r_{c}}\right)\right]+\sqrt{\frac{b}{r_{c}}\left(2-\frac{b}{r_{c}}\right)}\right)\right\} \\
& =\pi r_{c}-r_{c}\left[\frac{\pi}{2} \cos \theta+\cos \theta \arcsin (\cos \theta)+\sqrt{(1-\cos \theta)(1+\cos \theta)}\right] \quad\left(\cos \theta=1-\frac{b}{r_{c}}\right) \\
& =\pi r_{c}-r_{c}\left[\frac{\pi}{2} \cos \theta+\cos \theta\left(\frac{\pi}{2}-\arccos (\cos \theta)\right)+\sin \theta\right] \\
& =r_{c}\left[\pi-\left(\frac{\pi}{2} \cos \theta+\frac{\pi}{2} \cos \theta\right)+\arccos (\cos \theta) \cos \theta-\sin \theta\right] \\
& =r_{c}[\pi(1-\cos \theta)+\theta \cos \theta-\sin \theta] \\
& =r_{c}\left[\pi \frac{b}{r_{c}}-(\sin \theta-\theta \cos \theta)\right]\left(1-\cos \theta=\frac{b}{r_{c}}\right) \cdot
\end{aligned}
$$

conforming the results in equations (3.18) and (3.19). And for the expression of $\mu_{\text {reg }}$ provided in equation (4.12)

$$
\begin{aligned}
\mu_{\mathrm{reg}} & =-\frac{1}{2 b} \int_{0}^{b} d y \int_{\pi-\alpha(y)}^{\pi+\alpha(y)} \cos \varphi d \varphi \quad\left(\alpha(y)=\arccos \left[1-\frac{y}{r_{c}}\right]\right) \\
& =\frac{1}{b} \int_{0}^{b} d y \sin \left[\arccos \left(1-\frac{y}{r_{c}}\right)\right] \\
& =\frac{1}{b} \int_{0}^{b} d y \sqrt{1-\left(1-\frac{y}{r_{c}}\right)^{2}}=-\frac{r_{c}}{b} \int_{1}^{1-b / r_{c}} \sqrt{1-t^{2}} d t \quad\left(t=1-\frac{y}{r_{c}}\right) \\
& =\frac{r_{c}}{2 b}(\theta-\cos \theta \sin \theta)=\frac{1}{2} \frac{\theta-\cos \theta \sin \theta}{1-\cos \theta} \quad\left(\frac{b}{r_{c}}=1-\cos \theta\right) .
\end{aligned}
$$




\section{Appendix D}

\section{Solution of the Biased Diffusion Equation}

The biased diffusion equation is given by

$$
\frac{\partial P(x, t)}{\partial t}+v \frac{\partial P(x, t)}{\partial x}=\frac{D}{2} \frac{\partial^{2} P(x, t)}{\partial x^{2}} .
$$

In order to solve this equation we introduce the Fourier transform of the concentration

$$
P(k, t)=\int_{-\infty}^{\infty} P(x, t) e^{i k x} d x
$$

Then multiplying equation (D.1) by $e^{i k x}$ and integrating over $x$, will provide

$$
\int_{-\infty}^{\infty} \frac{\partial P(x, t)}{\partial t} e^{i k x} d x+v \int_{-\infty}^{\infty} \frac{\partial P(x, t)}{\partial x} e^{i k x} d x=\frac{D}{2} \int_{-\infty}^{\infty} \frac{\partial^{2} P(x, t)}{\partial x^{2}} e^{i k x} d x
$$

We note that

$$
\int \frac{\partial P(x, t)}{\partial t} e^{i k x} d x=\frac{\partial P(k, t)}{\partial t}, \quad \int \frac{\partial P(x, t)}{\partial x} e^{i k x} d x=P(x, t) e^{i k x}-i k \int P(x, t) e^{i k x} d x,
$$

and

$$
\int \frac{\partial^{2} P(x, t)}{\partial x^{2}} e^{i k x} d x=\frac{\partial P(x, t)}{\partial x} e^{i k x}-i k P(x, t) e^{i k x}-k^{2} \int P(x, t) e^{i k x} d x,
$$

Therefore for fast decay of probabilities as $x \rightarrow \pm \infty$, we can simplify the biased diffusion equation to

$$
\frac{\partial P(k, t)}{\partial t}=\left(i k v-\frac{D}{2} k^{2}\right) P(k, t) .
$$

Now it's easy to solve this equation, with the initial condition $P(x, t=0)=\delta(x)$, to obtain

$$
P(k, t)=P(k, 0) \exp \left[\left(i k v-\frac{D}{2} k^{2}\right) t\right]=\exp \left[\left(i k v-\frac{D}{2} k^{2}\right) t\right],
$$


Now we can invert the the Fourier transform to get back the probability distribution

$$
P(x, t)=\frac{1}{2 \pi} \int_{-\infty}^{\infty} \exp \left[\left(i k v-\frac{D}{2} k^{2}\right) t-i k x\right] d k .
$$

In order to solve this integration we note that

$$
\left(i k v-\frac{D}{2} k^{2}\right) t-i k x=-\frac{D}{2} t\left[k-\frac{i(v t-x)}{D t}\right]^{2}-\frac{(v t-x)^{2}}{2 D t} .
$$

Then the integration in equation (D.6) results in a Gaussian form of the probability distribution

$$
\begin{aligned}
P(x, t) & =\frac{1}{2 \pi} \exp \left[-\frac{(v t-x)^{2}}{2 D t}\right] \int_{-\infty}^{\infty} \exp \left[-\frac{D}{2} t\left(k-i \frac{v t-x}{D t}\right)^{2}\right] d k \\
& =\frac{1}{\sqrt{2 \pi D t}} \exp \left[-\frac{(x-v t)^{2}}{2 D t}\right] .
\end{aligned}
$$




\section{Appendix E}

\section{Exit Probabilities for Biased Random Walk}

We will calculate exit probabilities using the electrostatics approach. According to this approach [74], "For a diffusing particle that is initially at $\vec{r}_{0}$ inside a domain with absorbing boundary conditions, the eventual hitting probability to a boundary point $\vec{r}_{B}$ equals the electric field at this same location when a point charge of magnitude $1 /\left(D \Omega_{d}\right)$ is placed at $\vec{r}_{0}$ and the domain boundary is grounded"; where, $D$ is the diffusion constant associated with the diffusing particle and $\Omega_{d}$ is the $d$-dimensional volume element associated with the dimension of the problem.

To apply this formalism, we integrate the FPE over all time

$$
\int_{0}^{\infty} \frac{\partial P(x, t)}{\partial t} d t=-v \frac{d}{d x} \int_{0}^{\infty} P(x, t) d t+\frac{D}{2} \frac{d^{2}}{d x^{2}} \int_{0}^{\infty} P(x, t) d t
$$

with the initial condition that at time $t=0$, the particle is at $x=x_{0}$ within the system (see figure E.1).

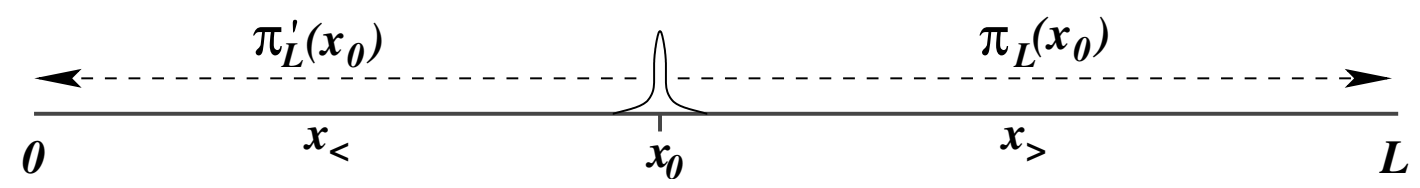

Figure E.1: To calculate the exit probabilities, the particle starts its motion from $x=x_{0}$ at time $t=0$ and then moves to either of the directions. Depending on the direction of motion of the particle the exit probabilities will be different.

We now consider spatial domains for which eventual absorption is certain i.e.,

$$
\lim _{t \rightarrow \infty} P(x, t)=0 .
$$


This reduces the above equation to

$$
\frac{D}{2} \frac{d^{2} C}{d x^{2}}-v \frac{d C}{d x}=-\delta\left(x-x_{0}\right),
$$

where $C(x)$ is the time integrated density

$$
C(x)=\int_{0}^{\infty} P(x, t) d t .
$$

For solving the equation (E.3) with (i) the boundary condition that the particle gets absorbed at the boundary, $C(0)=C(L)=0$ and (ii) the initial condition that at time $t=0$ the diffusing particle is at position $x=x_{0}, P(x, 0)=\delta\left(x-x_{0}\right)$; we assume, for $x \neq x_{0}$, a solution of the form $C(x)=A \exp (\alpha x)$. Substituting this back into equation (E.3), we obtain

$$
\frac{D}{2} \alpha^{2}-v \alpha=0
$$

Solving it provides $\alpha=0$ or $\alpha=2 v / D=1 / \lambda$. Therefore, the solutions can be written as

$$
C_{0}=1 \quad \text { and } \quad C_{1}=\exp (x / \lambda)
$$

In general, a combination of both of them is also a solution of the original equation. Therefore, for the boundary value problem we assume a solution of the form

$$
C=a+b \exp (x / \lambda)
$$

with $a$ and $b$ being some constants. Now the boundary condition that $C(0)=0$ and $C(L)=0$ says that

$$
a+b=0 \quad(x=0)
$$

and

$$
a+b \exp (L / \lambda)=0 \quad(x=L) .
$$

With these equations we can write the Green's function in the region $x<x_{0}\left(C_{<}(x)\right)$ and in the region $x>x_{0}\left(C_{>}(x)\right)$ as

$$
C_{<}(x)=a_{<}[1-\exp (x / \lambda)]
$$

and

$$
C_{>}(x)=a_{>}[1-\exp ((x-L) / \lambda)] .
$$

where $a_{<}$and $a_{>}$are constants. To determine these constants we use the continuity of the solution at 
$x=x_{0}$. This provides

$$
a_{<}\left[1-\exp \left(\frac{x_{0}}{\lambda}\right)\right]=a_{>}\left[1-\exp \left(\frac{x_{0}-L}{\lambda}\right)\right] .
$$

giving a relation between the ratios of $a_{<}$to $a_{>}$. Using this we can write the solutions in terms of an unknown constant $A$ as

$$
\begin{aligned}
& C_{<}(x)=A\left[1-\exp \left(\frac{x_{0}-L}{\lambda}\right)\right]\left[1-\exp \left(\frac{x}{\lambda}\right)\right] \\
& C_{>}(x)=A\left[1-\exp \left(\frac{x_{0}}{\lambda}\right)\right]\left[1-\exp \left(\frac{x-L}{\lambda}\right)\right] .
\end{aligned}
$$

Now to determine the last constant we integrate the equation $(\overline{E .3})$ on a small region $\epsilon$ about $x_{0}$ : $\left\{x_{0}-\epsilon, x_{0}+\epsilon\right\}$ to find

$$
\frac{D}{2}\left(\left.\frac{d C}{d x}\right|_{x_{0}+\epsilon}-\left.\frac{d C}{d x}\right|_{x_{0}-\epsilon}\right)-v\left(\left.C\right|_{x_{0}+\epsilon}-\left.C\right|_{x_{0}-\epsilon}\right)=-1
$$

Continuity of the solution at $x=0$ suggests that $\left.C\right|_{x_{0}+\epsilon}-\left.C\right|_{x_{0}-\epsilon}=0$, therefore,

$$
\left.\frac{d C_{<}(x)}{d x}\right|_{x_{0}}-\left.\frac{d C_{>}(x)}{d x}\right|_{x_{0}}=-\frac{2}{D}
$$

Solving this yields

$$
A=\frac{-1}{v \exp \left(x_{0} / \lambda\right)[1-\exp (-L / \lambda)]}
$$

So, the solution takes the form

$$
C(x)=-\frac{1}{v} \frac{\left[1-e^{x_{<} / \lambda}\right]\left[1-e^{\left(x_{>}-L\right) / \lambda}\right]}{e^{x_{0} / \lambda}\left[1-e^{-L / \lambda}\right]} .
$$

where $x_{<}=\min \left(x, x_{0}\right)$ and $x_{>}=\max \left(x, x_{0}\right)$. Now we can calculate the flux to the boundary $x=0$ or to $x=L$ to find the exit probabilities

$$
\begin{gathered}
\pi_{L}^{\prime}\left(x_{0}\right)=\left.\left[-v C(x)+D \frac{d C(x)}{d x}\right]\right|_{x=0}=\frac{e^{-x_{0} / \lambda}-e^{-L / \lambda}}{1-e^{-L / \lambda}} \\
\pi_{L}\left(x_{0}\right)=\left.\left[v C(x)-D \frac{d C(x)}{d x}\right]\right|_{x=L}=\frac{1-e^{-x_{0} / \lambda}}{1-e^{-L / \lambda}}
\end{gathered}
$$

reproducing the results we have used for our problem. 



\section{Appendix F}

\section{Relation between components of scattering matrix and transfer matrix}

Consider the scattering process shown in figure F.1 then the incoming probabilities can be related to the outgoing probabilities by the equations

$$
\begin{aligned}
& b_{-}=t a_{-}+r^{\prime} b_{+} \\
& a_{+}=r a_{-}+t^{\prime} b_{+},
\end{aligned}
$$

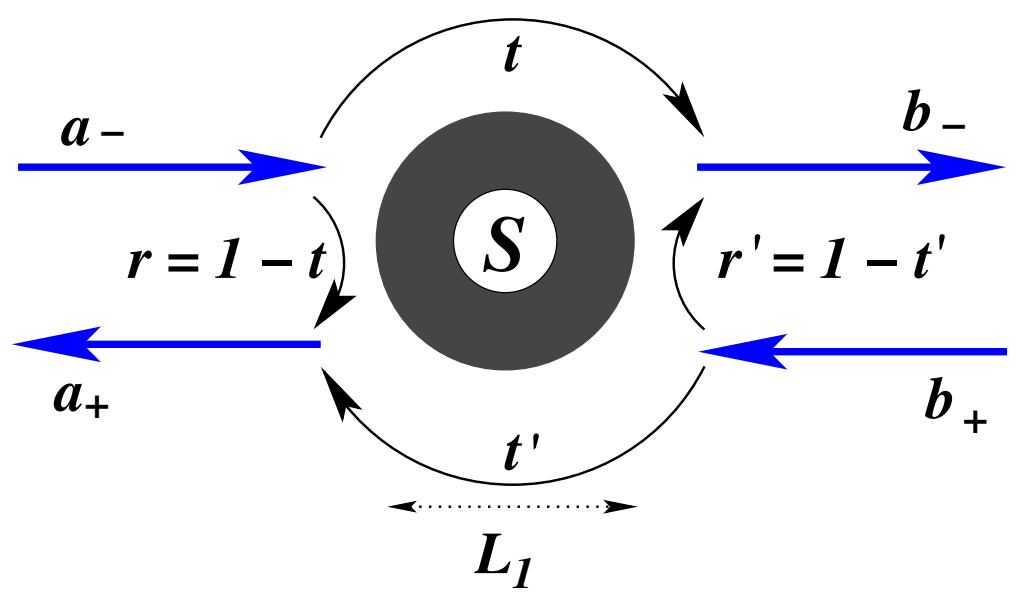

Figure F.1: Scattering model for BPRW describing transmission probabilities. The outgoing probabilities $\left(a_{+}, b_{-}\right)$relate themselves to the incoming ones $\left(a_{-}, b_{+}\right)$using a scattering matrix (equation (F.3)). This can be modified to relate the right hand probabilities $\left(b_{-}, b_{+}\right)$with the left hand probabilities $\left(a_{-}, a_{+}\right)$using a transfer matrix (equation (F.6) $)$. 
or in matrix form as

$$
\left(\begin{array}{l}
a_{+} \\
b_{-}
\end{array}\right)=S\left(\begin{array}{c}
a_{-} \\
b_{+}
\end{array}\right)=\left(\begin{array}{ll}
r & t^{\prime} \\
t & r^{\prime}
\end{array}\right)\left(\begin{array}{l}
a_{-} \\
b_{+}
\end{array}\right)
$$

where $S$ is the scattering matrix for the scattering process depicted in figure F.1. Now from equation (F.2) we can write

$$
\begin{aligned}
b_{+} & =\frac{1}{t^{\prime}} a_{+}-\frac{r}{t^{\prime}} a_{-} \\
& =\frac{1}{t^{\prime}} a_{+}+\frac{t-1}{t^{\prime}} a_{-}
\end{aligned}
$$

Using this equation we can write from equation (F.1)

$$
\begin{aligned}
b_{-} & =t a_{-}+r^{\prime} b_{+} \\
& =t a_{-}+r^{\prime}\left(\frac{1}{t^{\prime}} a_{+}+\frac{t-1}{t^{\prime}} a_{-}\right) \\
& =\frac{1-t^{\prime}}{t^{\prime}} a_{+}+\left(t+r^{\prime} \frac{t-1}{t^{\prime}}\right) a_{-} \\
& =\frac{1-t^{\prime}}{t^{\prime}} a_{+}+\frac{t t^{\prime}+\left(1-t^{\prime}\right)(t-1)}{t^{\prime}} a_{-} \\
& =\frac{1-t^{\prime}}{t^{\prime}} a_{+}+\frac{t+t^{\prime}-1}{t^{\prime}} a_{-}
\end{aligned}
$$

The equations (F.4) and (F.5) can be rewritten in terms of a matrix form (providing the transfer matrix $T)$ as

$$
\left(\begin{array}{c}
b_{-} \\
b_{+}
\end{array}\right)=T\left(\begin{array}{l}
a_{-} \\
a_{+}
\end{array}\right)=\left(\begin{array}{cc}
\frac{t+t^{\prime}-1}{t^{\prime}} & \frac{1-t^{\prime}}{t^{\prime}} \\
\frac{-(1-t)}{t^{\prime}} & \frac{1}{t^{\prime}}
\end{array}\right)\left(\begin{array}{l}
a_{-} \\
a_{+}
\end{array}\right) .
$$

conforming the result in equation 4.58 . 


\section{Appendix G}

\section{Transmission probabilities using the transfer matrix}

As can be seen the final step of multiplying $n$ transfer matrices (refer to equation (4.63)) is a tedious job as the equations brings in more and more complications with each multiplication. However, there is an easier way. It is to find the eigenvalues and eigenvectors of matrix $T$. For the transfer matrix in equation (4.58), the eigenvalues and the corresponding eigenvectors can be found by solving for $\xi$ in the equation

$$
\operatorname{Det}(T-\xi I)=0
$$

where $I$ is the identity matrix of the same dimension as that of the matrix $T$. Solving this equation yields the eigenvalues $\xi_{1(2)}$ and the corresponding eigenvectors $V_{1(2)}$. They are

$$
\begin{array}{ll}
\xi_{1}=1, & V_{1}=\left(\begin{array}{c}
\frac{1-t^{\prime}}{1-t} \\
1
\end{array}\right) \\
\xi_{2}=\frac{t}{t^{\prime}}, & V_{2}=\left(\begin{array}{c}
1 \\
1
\end{array}\right) .
\end{array}
$$

For right to left transmission we need

$$
a_{0}=\left(\begin{array}{l}
0 \\
1
\end{array}\right)=\frac{1-t}{t^{\prime}-t} V_{1}+\frac{1-t^{\prime}}{t-t^{\prime}} V_{2}
$$


Operating $T_{n}$ from equation (4.63), we will obtain

$$
\begin{aligned}
T_{n}\left(\begin{array}{l}
0 \\
1
\end{array}\right) & =\underbrace{T T \ldots T T}_{\mathrm{n} \text {-terms }}\left[\frac{1-t}{t^{\prime}-t} V_{1}+\frac{1-t^{\prime}}{t-t^{\prime}} V_{2}\right] \\
& =E_{1}^{n} \frac{1-t}{t^{\prime}-t} V_{1}+E_{2}^{n} \frac{1-t^{\prime}}{t-t^{\prime}} V_{2} \\
& =\frac{1-t}{t^{\prime}-t}\left(\begin{array}{c}
\frac{1-t^{\prime}}{1-t} \\
1
\end{array}\right)+\left(\frac{t}{t^{\prime}}\right)^{n} \frac{1-t^{\prime}}{t-t^{\prime}}\left(\begin{array}{l}
1 \\
1
\end{array}\right) \\
& =\left(\begin{array}{c}
\frac{1-t_{n}^{\prime}}{t_{n}^{\prime}} \\
\frac{1}{t_{n}^{\prime}}
\end{array}\right)=\left(\begin{array}{l}
T_{n}^{1,2} \\
T_{n}^{2,2}
\end{array}\right),
\end{aligned}
$$

using equation (4.59) and after inserting the values of $\xi_{1(2)}$ and $V_{1(2)}$ from equations (G.2) and (G.3). We can solve for $t_{n}^{\prime}$ by using the components of $T_{n}$

$$
\frac{T_{n}^{1,2}}{T_{n}^{2,2}}=1-t_{n}^{\prime} \Rightarrow t_{n}^{\prime}=1-\frac{T_{n}^{1,2}}{T_{n}^{2,2}} .
$$

As the final step, we substitute the components $T_{n}^{1,2}$ and $T_{n}^{2,2}$ from equation (G.5) into equation (G.7). This yields

$$
t_{n}^{\prime}=1-\frac{\frac{1-t^{\prime}}{t^{\prime}-t}+\left(\frac{t}{t^{\prime}}\right)^{n} \frac{1-t^{\prime}}{t-t^{\prime}}}{\frac{1-t}{t^{\prime}-t}+\left(\frac{t}{t^{\prime}}\right)^{n} \frac{1-t^{\prime}}{t-t^{\prime}}}=\frac{1-\frac{1-t}{1-t^{\prime}}}{\left(\frac{t}{t^{\prime}}\right)^{n}-\frac{1-t}{1-t^{\prime}}}=\frac{1-\frac{r}{r^{\prime}}}{\left(\frac{t}{t^{\prime}}\right)^{n}-\frac{r}{r^{\prime}}} .
$$

Similarly, for left to right transmission, we assume the initial probability

$$
a_{0}=\left(\begin{array}{l}
1 \\
0
\end{array}\right)=\frac{1-t}{t-t^{\prime}} V_{1}+\frac{1-t}{t^{\prime}-t} V_{2}
$$


to obtain

$$
\begin{aligned}
T_{n}\left(\begin{array}{l}
1 \\
0
\end{array}\right) & =\left(\begin{array}{c}
\frac{t_{n}+t_{n}^{\prime}-1}{t_{n}^{\prime}} \\
\frac{-\left(1-t_{n}\right)}{t_{n}^{\prime}}
\end{array}\right)=\left(\begin{array}{c}
T_{n}^{1,1} \\
T_{n}^{2,1}
\end{array}\right) \\
& =\underbrace{T T \ldots T T}_{\mathrm{n} \text {-terms }}\left[\frac{1-t}{t-t^{\prime}} V_{1}+\frac{1-t}{t^{\prime}-t} V_{2}\right] \\
& =\frac{1-t}{t-t^{\prime}}\left(\begin{array}{c}
1-t^{\prime} \\
1-t \\
1
\end{array}\right)+\left(\frac{t}{t^{\prime}}\right)^{n} \frac{1-t}{t^{\prime}-t}\left(\begin{array}{c}
1 \\
1
\end{array}\right) .
\end{aligned}
$$

This will provide us a relation between $t_{n}$ and $t_{n}^{\prime}$ as

$$
\frac{T_{n}^{1,1}}{T_{n}^{2,1}}=\frac{t_{n}+t_{n}^{\prime}-1}{t_{n}-1}
$$

which solves to

$$
\begin{aligned}
t_{n} & =1+\frac{t_{n}^{\prime}}{\frac{T_{n}^{1,1}}{T_{n}^{2,1}}-1}=1+\frac{t_{n}^{\prime}}{\frac{\left(\frac{1-t}{t-t^{\prime}}\right)\left(\frac{1-t^{\prime}}{1-t}\right)+\left(\frac{t}{t^{\prime}}\right)^{n}\left(\frac{1-t}{t^{\prime}-t}\right)}{\left(\frac{1-t}{t-t^{\prime}}\right)+\left(\frac{t}{t^{\prime}}\right)^{n}\left(\frac{1-t}{t^{\prime}-t}\right)}-1} \\
& =1+t_{n}^{\prime}\left(\frac{1-t}{t^{\prime}-t}\right)\left[\left(\frac{t}{t^{\prime}}\right)^{n}-1\right] .
\end{aligned}
$$

In order to simplify this equation further, we note from equation (G.8) that $t_{n}^{\prime}$ satisfy

$$
t_{n}^{\prime}\left[\left(\frac{t}{t^{\prime}}\right)^{n}-1\right]=\left(\frac{t-t^{\prime}}{1-t^{\prime}}\right)\left(1-t_{n}^{\prime}\right) \quad \text { or } \quad t_{n}^{\prime}\left(\frac{1-t}{t^{\prime}-t}\right)\left[\left(\frac{t}{t^{\prime}}\right)^{n}-1\right]=\left(\frac{1-t}{1-t^{\prime}}\right)\left(t_{n}^{\prime}-1\right) .
$$

Therefore, we rewrite the expression for $t_{n}$ from equation (G.12) as

$$
t_{n}=1+\left(\frac{1-t}{1-t^{\prime}}\right)\left(t_{n}^{\prime}-1\right)=\left(1-\frac{1-t}{1-t^{\prime}}\right)+\frac{1-t}{1-t^{\prime}} t_{n}^{\prime}=\frac{t-t^{\prime}}{1-t^{\prime}}+\frac{1-t}{1-t^{\prime}} t_{n}^{\prime}
$$

Now we substitute the value of $t_{n}^{\prime}$ from equation (G.8) into equation (G.14) to obtain an expression of $t_{n}$ in terms of $t$ and $t^{\prime}$ as

$$
t_{n}=\frac{\frac{t^{\prime}-t}{1-t}}{\left(\frac{t^{\prime}}{t}\right)^{n}-\frac{1-t^{\prime}}{1-t}} \cdot=\frac{1-\frac{r^{\prime}}{r}}{\left(\frac{t^{\prime}}{t}\right)^{n}-\frac{r^{\prime}}{r}} .
$$





\section{Appendix H}

\section{BPRW - A discrete approach}

We will use the scattering matrix in order to calculate the various contributions to the phase space. The structure of phase space suggests that the scattering matrix associated with our system is given by

$$
S=\left(\begin{array}{cc}
r & t^{\prime} \\
t & r^{\prime}
\end{array}\right)
$$

with $r+t=r^{\prime}+t^{\prime}=1$ for conservation of probability. In the infinite system if the fraction of forward and backward movers is $f_{ \pm}$, then within one scattering they are related by

$$
\left(\begin{array}{c}
f_{-}^{t+1} \\
f_{+}^{t+1}
\end{array}\right)=S\left(\begin{array}{c}
f_{+}^{t} \\
f_{-}^{t}
\end{array}\right)
$$

where, $f_{ \pm}^{t}$ are the fraction of backward and forwards movers at time $t$. The above matrix equations can be rewritten as

$$
\left(\begin{array}{c}
f_{+}^{t+1} \\
f_{-}^{t+1}
\end{array}\right)=\left(\begin{array}{ll}
t & r^{\prime} \\
r & t^{\prime}
\end{array}\right)\left(\begin{array}{c}
f_{+}^{t} \\
f_{-}^{t}
\end{array}\right)
$$

The eigenvalues and the eigenvectors of this scattering matrix are given by

$$
\begin{array}{cc}
\xi_{1}=1, \quad V_{1}=\left(\begin{array}{c}
\frac{r^{\prime}}{r} \\
1
\end{array}\right), \\
\xi_{2}=\left(1-r-r^{\prime}\right), \quad V_{2}=\left(\begin{array}{c}
-1 \\
1
\end{array}\right)
\end{array}
$$

The constancy of the chaotic phase space volume says that for all $t$

$$
f_{+}+f_{-}=1
$$


We can solve the scattering matrix to obtain

$$
\begin{aligned}
& f_{+}^{t+1}=t f_{+}^{t}+r^{\prime} f_{-}^{t} \\
& f_{-}^{t+1}=r f_{+}^{t}+t^{\prime} f_{-}^{t}
\end{aligned}
$$

We are looking for stationary solutions i.e., when the scattering is one to one and area preserving, then $f_{+}^{t+1}=f_{+}^{t}$ and from the fact that the total chaotic phase space area is $f_{+}+f_{-}=1$ we get a solution for the stationary states $f_{+}^{s}$ and $f_{-}^{s}$ as

$$
\begin{aligned}
f_{+}^{s} & =\frac{r^{\prime}}{r+r^{\prime}}, \\
f_{-}^{s} & =\frac{r}{r+r^{\prime}} .
\end{aligned}
$$

This is the property of the first eigenvector $V_{1}$, whose eigenvalue is just unity. This can be shown by writing

$$
\left(\begin{array}{c}
\frac{r^{\prime}}{r+r^{\prime}} \\
\frac{r}{r+r^{\prime}}
\end{array}\right)=\left(\frac{r}{r+r^{\prime}}\right)\left(\begin{array}{c}
\frac{r^{\prime}}{r} \\
1
\end{array}\right)
$$

In the spirit of sum rule the different occupations $f_{ \pm}$are related to different available phase spaces of the chaotic trajectories. Therefore, the chaotic velocity which is given by the relative phase space area of the chaotic trajectories in the stationary state will be

$$
v_{\mathrm{ch}}=\frac{f_{+}-f_{-}}{f_{+}+f_{-}}=\frac{r^{\prime}-r}{r^{\prime}+r},
$$

in confirmation with the relation (4.45) (for dimensionality reasons this must be multiplied with $v=$ $\Delta x / \Delta l)$. However, the entire phase space of the system should be balanced. So, we postulate a regular component of the phase space with volume $R$ (see figure H.1) such that

$$
\begin{aligned}
R+C & =1 \\
R+f_{-} C & =f_{+} C
\end{aligned}
$$

where, $C$ is the total volume of the chaotic phase space. As the regular trajectories does not get scattered an any moment of time, the dynamics in 1D walk suggest that they always have to move in backward direction with velocity $v_{R}=-1$. At any moment the net current inside the system must vanish. Therefore the equilibrium condition becomes

$$
R+f_{-} C=f_{+} C,
$$




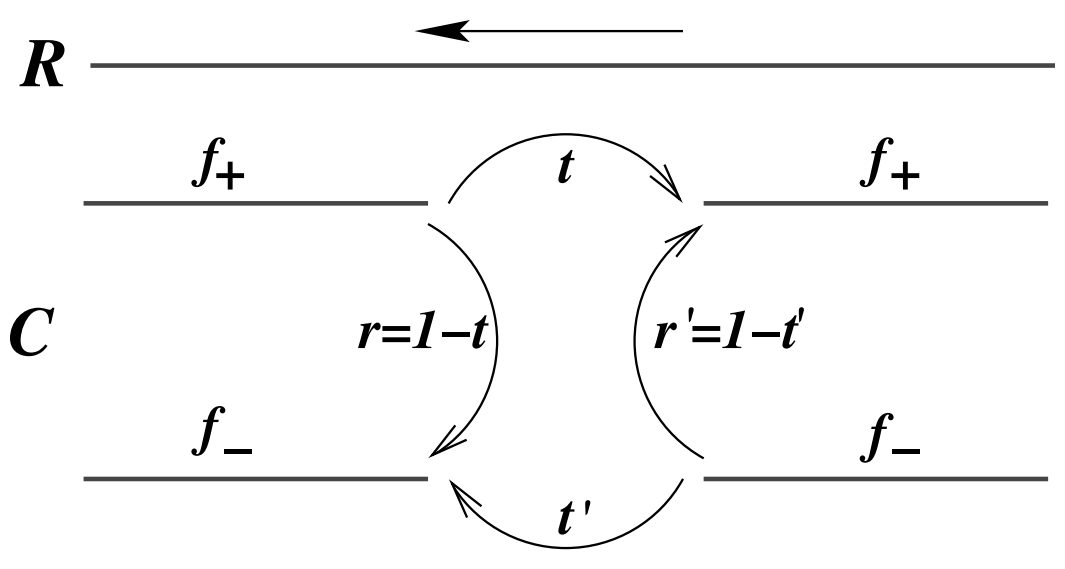

Figure H.1: The persistent random walk and the phase space dynamics. $R$ is the fraction of regular trajectories which does not get scattered and move in a single direction (backward motion). $C$ is the fraction of chaotic trajectories out of which $f_{+}$is the fraction of chaotic particles which move in forward direction and $f_{-}$is the fraction of particles moving in the negative direction. The resultant bias of the chaotic trajectories is then given by $f_{+}-f_{-}$in stationary state.

in other word the total current in the backward direction which is $R+f_{-} C$ must equal to the current in the forward direction $f_{+} C$. We can solve this equation to obtain in the stationary state

$$
\begin{aligned}
& C=\frac{r+r^{\prime}}{2 r^{\prime}} \\
& R=\frac{r^{\prime}-r}{2 r^{\prime}}
\end{aligned}
$$

Since, the total fraction of particles moving in each direction is equal to $R+f_{-} C=f_{+} C$ out of which $R$ numbers would saturate to infinity, the asymptotic value of transmission will be given by

$$
t_{\infty}=\frac{R}{f_{+} C}=\frac{\frac{r^{\prime}-r}{2 r^{\prime}}}{\left(\frac{r^{\prime}}{r+r^{\prime}}\right)\left(\frac{r+r^{\prime}}{2 r^{\prime}}\right)}=\frac{r^{\prime}-r}{r^{\prime}}=1-\frac{r}{r^{\prime}}
$$

in conformity with relation (4.69). The rest remained is to calculate the diffusion constant based on the relaxation mechanism, defined by the other eigenvalue of the scattering matrix. Within the relaxation mechanism we can prepare the initial state

$$
a_{+, 0}=\left(\begin{array}{l}
1 \\
0
\end{array}\right)
$$


for transmission in the + ve direction. This can be written in terms of the eigenvector $V_{2}$ and the stationary states $f_{ \pm}^{s}$ as

$$
a_{+, 0}\left(\begin{array}{l}
1 \\
0
\end{array}\right)=\left(\begin{array}{c}
f_{+}^{s} \\
f_{-}^{s}
\end{array}\right)-f_{-}^{s}\left(\begin{array}{c}
-1 \\
1
\end{array}\right)=\left(\begin{array}{c}
f_{+}^{s} \\
f_{-}^{s}
\end{array}\right)-f_{-}^{s} V_{2} .
$$

Similarly for the negative direction of transport we can define an initial state

$$
a_{-, 0}=\left(\begin{array}{l}
0 \\
1
\end{array}\right)=\left(\begin{array}{c}
f_{+}^{s} \\
f_{-}^{s}
\end{array}\right)+f_{+}^{s}\left(\begin{array}{c}
-1 \\
1
\end{array}\right)=\left(\begin{array}{c}
f_{+}^{s} \\
f_{-}^{s}
\end{array}\right)+f_{+}^{s} V_{2} \text {. }
$$

Therefore, at any other time $n$, we can write the evolution of these states as

$$
\begin{aligned}
a_{+, n} & =\underbrace{S S \ldots S S}_{\mathrm{n}-\text { times }} a_{+, 0} \\
& =\left(\begin{array}{c}
f_{+}^{s} \\
f_{-}^{s}
\end{array}\right)-\left(1-r-r^{\prime}\right)^{n} f_{-}^{s}\left(\begin{array}{c}
-1 \\
1
\end{array}\right) .
\end{aligned}
$$

Similarly, for the state $a_{-, n}$

$$
a_{-, n}=\left(\begin{array}{c}
f_{+}^{s} \\
f_{-}^{s}
\end{array}\right)+\left(1-r-r^{\prime}\right)^{n} f_{+}^{s}\left(\begin{array}{c}
-1 \\
1
\end{array}\right) .
$$

Therefore, the velocity correlation function can be calculated as

$$
\begin{aligned}
\left\langle v_{0} v_{n}\right\rangle= & +\left[(1) f_{+}^{s}-(0) f_{-}^{s}\right]\left[\left(f_{+}^{s}-\left(1-r-r^{\prime}\right)^{n} f_{-}^{s}(-1)\right)-\left(f_{-}^{s}-\left(1-r-r^{\prime}\right)^{n} f_{-}^{s}(1)\right)\right] \\
& +\left[f_{+}^{s}(0)-(1) f_{-}^{s}\right]\left[\left(f_{+}^{s}+\left(1-r-r^{\prime}\right)^{n} f_{+}^{s}(-1)\right)-\left(f_{-}^{s}+\left(1-r-r^{\prime}\right)^{n} f_{+}^{s}(1)\right)\right] \\
= & f_{+}^{s}\left[v_{\mathrm{ch}}+2\left(1-r-r^{\prime}\right)^{n} f_{-}^{s}\right]-f_{-}^{s}\left[v_{\mathrm{ch}}-2\left(1-r-r^{\prime}\right)^{n} f_{+}^{s}\right] \\
= & v_{c h}^{2}+4\left(1-r-r^{\prime}\right)^{n} f_{+}^{s} f_{-}^{s} .
\end{aligned}
$$


Now the diffusion constant associated with the problem can be computed using the Green-Kubo formula

$$
\begin{aligned}
D & =\sum_{n=-\infty}^{\infty}\left\langle\left[v_{0}-v_{\mathrm{ch}}\right]\left[v_{n}-v_{\mathrm{ch}}\right]\right\rangle \\
& =\sum_{n=-\infty}^{\infty}\left(\left\langle v_{0} v_{n}\right\rangle-v_{\mathrm{ch}}^{2}\right) \\
& =4 f_{+} f_{-} \sum_{n=-\infty}^{\infty}\left(1-r-r^{\prime}\right)^{n} \\
& =4 f_{+} f_{-}\left(-1+2 \sum_{n=0}^{\infty}\left(1-r-r^{\prime}\right)^{n}\right) \\
& =4 f_{+} f_{-}\left(\frac{2}{1-\left(1-r-r^{\prime}\right)}-1\right) \\
& =\frac{8 r r^{\prime}}{\left(r+r^{\prime}\right)^{3}}-\frac{4 r r^{\prime}}{\left(r+r^{\prime}\right)^{2}} .
\end{aligned}
$$

In the limit of $r, r^{\prime} \rightarrow 0$, the first term in the diffusion equation dominates over the second term and we recover the result of continuum limit derived in equation (4.54). And in the limit of $r=r^{\prime}$ or the PRW we find the result for diffusion constant as

$$
D=\frac{1}{r}-1=\frac{1-r}{r}=\frac{t}{1-t},
$$

which has been used to obtain Ohm's law in equation (4.92). 



\section{Appendix I}

\section{Laplace transform solution for the FPP}

The convection-diffusion equation is given by

$$
\frac{\partial P(x, t)}{\partial t}+v \frac{\partial P(x, t)}{\partial x}=\frac{D}{2} \frac{\partial^{2} P(x, t)}{\partial x^{2}}
$$

To solve this for the Laplace transform of the probability distribution, with the initial condition $P(x, t=$ $0)=\delta\left(x-x_{0}\right)$ and the boundary conditions $P(x, 0)=P(x, L)=0$, we perform a Laplace transform to obtain

$$
\begin{aligned}
\int_{0}^{\infty} e^{-s t} \frac{\partial P(x, t)}{\partial t} d t & +v \int_{0}^{\infty} e^{-s t} \frac{\partial P(x, t)}{\partial x} d t=\frac{D}{2} \int_{0}^{\infty} e^{-s t} \frac{\partial^{2} P(x, t)}{\partial x^{2}} d t \\
\Rightarrow s P(x, s)+v P^{\prime}(x, s) & =\frac{D}{2} P^{\prime \prime}(x, s),
\end{aligned}
$$

where the primes denote partial derivative with respect to the position variable $x$. Now its easy to solve the above Laplace transformed equation. We assume a solution in the form $P(x, s)=\beta e^{\alpha x}$ and plug this into equation (I.2). This provides a characteristic equation in $\alpha$

$$
\alpha^{2}-\alpha \frac{2 v}{D}-\frac{2 s}{D}=0
$$

which can be solved yielding

$$
\alpha_{ \pm}=\frac{1}{D}\left(v \pm \sqrt{v^{2}+2 D s}\right)=\frac{v}{D} \pm w(s)
$$

where, $w(s)=\frac{\sqrt{v^{2}+2 D s}}{D}$. Therefore, the elementary solution of the Laplace transformed equation can be written in the form

$$
P(x, s)=A_{+} e^{\alpha_{+} x}+A_{-} e^{\alpha_{-} x} .
$$




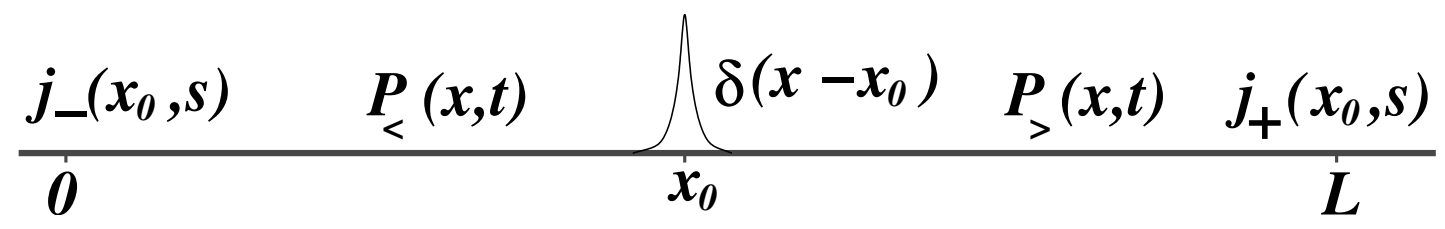

Figure I.1: The solution of the random walker for a finite channel of length $L$. The random walker starts its movement from a point $x_{0}$ at time $t=0$ within the channel. On both sides of $x_{0}$ the solutions are different. In the positions greater than the value of $x_{0}$ the solution is given by $P_{>}(x . t)$ whereas for smaller values $x<x_{0}$ the solution is given by $P_{<}(x . t)$. The Laplace transform of currents to each ends are denoted by $J_{+}\left(x_{0}, s\right)$ for forward movement to $x=L$ and $J_{-}\left(x_{0}, s\right)$ for the backward movement to $x=0$.

Now to obtain the coefficients $A_{ \pm}$we apply the boundary conditions $P(0, t)=P(L, t)=0$. For the boundary condition at $x=0$, we find $A_{+}=-A_{-}$and in the later case (i.e., for the boundary condition at $x=L$ ), we obtain $A_{+} e^{w(s) L}=-A_{-} e^{-w(s) L}$. Therefore for the two regions separated by the position $x=x_{0}$ (see figure I.1), we can assume solutions

$$
\begin{aligned}
P_{<}(x, s)=A_{+}\left(e^{\alpha_{+} x}-e^{\alpha_{-} x}\right) & =A e^{v x / D} \sinh [w(s) x], x<x_{0} \\
\text { and } P_{>}(x, s)=A_{+} e^{\alpha_{+} x}+A_{-} e^{\alpha_{-} x} & =A_{+}\left(e^{\alpha_{+} x}+\frac{A_{-}}{A_{+}} e^{\alpha_{-} x}\right) \\
\Rightarrow P_{>}(x, s)=A_{+}\left(e^{\alpha_{+} x}-e^{2 w(s) L} e^{\alpha_{-} x}\right) & =B e^{w(s) L} e^{v x / D} \sinh [w(s)(L-x)], x>x_{0} .
\end{aligned}
$$

The consequence of absorbing boundary conditions leads to the solutions having antisymmetric combinations of exponentials

$$
P(x, s)= \begin{cases}A e^{v x / D} \sinh [w(s) x] & \left(x<x_{0}\right) \\ B e^{w(s) L} e^{v x / D} \sinh [w(s)(L-x)] & \left(x>x_{0}\right),\end{cases}
$$

where $A$ and $B$ are constants. To obtain these constants we impose the continuity of solutions at $x=x_{0}$. This yields a relation between $A$ and $B$ as

$$
\begin{aligned}
A e^{v x_{0} / D} \sinh \left[w(s) x_{0}\right] & =B e^{w(s) L} e^{v x_{0} / D} \sinh \left[w(s)\left(L-x_{0}\right)\right] \\
\Rightarrow A & =B e^{w(s) L} \frac{\sinh \left[w(s)\left(L-x_{0}\right)\right]}{\sinh \left[w(s) x_{0}\right]}
\end{aligned}
$$


providing

$$
P(x, s)= \begin{cases}B e^{w(s) L} \frac{\sinh \left[w(s)\left(L-x_{0}\right)\right]}{\sinh \left[w(s) x_{0}\right]} e^{v x / D} \sinh [w(s) x] & \left(x<x_{0}\right) \\ B e^{w(s) L} e^{v x / D} \sinh [w(s)(L-x)] & \left(x>x_{0}\right) .\end{cases}
$$

The remaining constant $B$ can be found from the discontinuity of the first derivative of the concentration at the initial position $x=x_{0}$. For this purpose, we integrate the equation (I.2) over an infinitesimal interval around $x_{0}$, obtaining

$$
\int_{x_{0}-\epsilon}^{x_{0}+\epsilon} s P(x, s) d x+v \int_{x_{0}-\epsilon}^{x_{0}+\epsilon} P^{\prime}(x, s) d x=\frac{D}{2} \int_{x_{0}-\epsilon}^{x_{0}+\epsilon} P^{\prime \prime}(x, s) d x .
$$

The continuity of the probability density at $x_{0}$ says that the drift term in the integration vanishes in the limit $\epsilon \rightarrow 0$. Whereas the first term can be integrated using the fact that at time $t=0$ the particle is situated at $x=x_{0}$. This provides

$$
\int_{x_{0}-\epsilon}^{x_{0}+\epsilon} s P(x, s) d x=\int_{x_{0}-\epsilon}^{x_{0}+\epsilon} s \frac{-\delta\left(x-x_{0}\right)}{s} d x=-1
$$

Thus, the discontinuity in the wavefunction at $x_{0}$ takes the form

$$
\left.\frac{\partial P(x, s)}{\partial x}\right|_{x_{0}+\epsilon}-\left.\frac{\partial P(x, s)}{\partial x}\right|_{x_{0}-\epsilon}=-\frac{2}{D} .
$$

Using the values of the concentration from the equation (I.10), we can solve for $B$

$$
B=e^{-v x_{0} / D} \frac{\sinh \left[w(s) x_{0}\right]}{D w(s)\left[e^{2 w(s) L}-1\right]}=2 e^{-v x_{0} / D} e^{-w(s) L} \frac{\sinh \left[w(s) x_{0}\right]}{D w(s) \sinh [w(s) L]} .
$$

Substituting back into equation (I.10), we obtain the solution

$$
P(x, s)= \begin{cases}\frac{2 e^{v\left(x-x_{0}\right) / D}}{D w(s) \sinh [w(s) L]} \sinh [w(s) x] \sinh \left[w(s)\left(L-x_{0}\right)\right] & \left(x<x_{0}\right) \\ \frac{2 e^{v\left(x-x_{0}\right) / D}}{D w(s) \sinh [w(s) L]} \sinh \left[w(s) x_{0}\right] \sinh [w(s)(L-x)] & \left(x>x_{0}\right) .\end{cases}
$$

in conformity with the equation (5.8). 



\section{References}

[1] R. P. Feynman, R. B. Leighton, and M. Sands, The Feynman Lectures on Physics AddisonWesley (1966).

[2] G. Bar-Nahum et al, Cell 120183 (2005).

[3] R. D. Astumian and P. Hänggi, Physics Today 55(11) 33 (2002).

[4] J. S. Bader et al, PNAS (Biophysics) 9613165 (1999).

[5] R. D. Astumian, Science 276917 (1997).

[6] P. Reimann, Phys. Rep. 36157 (2002).

[7] F. Jülicher, A. Ajdari, and J. Prost, Rev. Mod. Phys. 691269 (1997).

[8] A. Adjari and J. Prost, C. R. Acad. Sci. Paris. II 3151635 (1992).

[9] M. O. Magnaso, Phys. Rev. Lett. 711477 (1993).

[10] R. D. Astumian and M. Bier Phys. Rev. Lett 721766 (1994).

[11] J. Prost. et al, Phys. Rev. Lett. 722652 (1994).

[12] J. Rousselet et al, Nature 370446 (1994).

[13] P. Reimann, Phys. Rep. 290149 (1997).

[14] R. Tammelo, R Mankin and D. Martila, Phys. Rev. E 66051101 (2002).

[15] H. Linke, Chaos 15026111 (2005).

[16] B. Ai, L. Wang and L. Liu, Phys. Rev. E 72031101 (2006).

[17] G. P. Harmer and D. Abbott, Stat. Sci. 14206 (1999).

[18] J. M. R. Parrondo, G. P. Harmer and D. Abbott, Phys. Rev. Lett. 845226 (2000). 
[19] M. Borromeo, G. Costantini and F. Marchesoni Phys. Rev. E 65041110 (2002).

[20] J. D. Meiss, Rev. Mod. Phys. 64795 (1992).

[21] G. M. Zaslavsky and S. S. Abdullaev, Phys. Rev. E 513901 (1995).

[22] S. S. Abdullaev and K. H. Spatschek, Phys. Rev. E 60 R6287 (1999).

[23] J. L. Mateos, Phys. rev. Lett., 84258 (2000).

[24] R. Artuso, I. Guarneri and L. Rebuzzini, Chaos 10189 (2000).

[25] O. Farago and Y. Kantor, Physica A 249151 (1998).

[26] S. Flach et al, Phys. Rev. Lett. 842358 (2000).

[27] H. Schanz et al, Phys. Rev. E 71026228 (2005).

[28] H. Schanz et al, Phys. Rev. Lett. 87070601 (2001).

[29] P. Reimann and P. Hänggi, Appl. Phys. A 75169 (2002).

[30] T. Dittrich, Phys. Rep. 271267 (1996).

[31] H. Linke et al, Europhys. Lett. 44343 (1998).

[32] I. Zapata et al, Phys. Rev. Lett. 772292 (1996).

[33] I. Zapata et al, Phys. Rev. Lett. 80802 (1998).

[34] F. Falo et al, Europhys. Lett. 45700 (1999).

[35] C. Mennerat-Robilliard, Phys. Rev. Lett. 82851 (1999).

[36] T. S. Monteiro et al, Phys. Rev. Lett. 89194102 (2002).

[37] C. S. Lee, Nature (London) 400337 (1999).

[38] S. Datta, Electronic Transport in Mesoscopic Systems Cambridge University Press (1999).

[39] P. A. M. Benistant et al, Phys. Rev. B 33690 (1986).

[40] H. Schanz and M. Prusty, J. Phys. A: Math. Gen. 3810085 (2005).

[41] M. Prusty and H. Schanz, Phys. Rev. Lett. 96130601 (2006).

[42] J. P. Bird, J. Phys. : Condens. Matter 11 R413 (1999). 
[43] L. P. Lévy et al, Physica B 189204 (1993).

[44] N. A. C. Hutchings et al, Phys. Rev. E 70036205 (2004).

[45] M. Horvat and T. Prosen, J. Phys. A: Math. Gen. 373133 (2004).

[46] S. Lepri et al, J. Stat. Phys. 99857 (2000).

[47] C. P. Dettmann et al, Nature (London) 401875 (1999).

[48] C. P. Dettmann and E. G. D. Cohen, J. Stat. Phys. 101775 (2000).

[49] B. Li, L. Wang and B. Hu, Phys. Rev. Lett. 88223901 (2002).

[50] R. Artuso, Physica D 1091 (1997).

[51] G. Casati and T. Prosen, Phys. Rev. Lett. 834729 (1999).

[52] D. Alonso, A. Ruiz and I. de Vega, Phys. Rev. E 66066131 (2002).

[53] G. M. Nikolopoulos et al, Europhys. Lett. 65297 (2004).

[54] A. Rahman et al, Phys. Rev. B 73125313 (2006).

[55] K. B. Efetov, Phys. Rev. Lett. 79491 (1997).

[56] K. Hornberger and U. Smilansky, Physics Reports 367249 (2002).

[57] S. J. Lee et al, Physics Reports 3941 (2004).

[58] S. Flach et al, Phys. Rev. Lett. 842358 (2000).

[59] S. Denisov et al, Physica D 170131 (2002).

[60] B. Friedman and R. F. Martin, Phys. Lett. A 10523 (1984).

[61] A. Zacherl et al, Phys. Lett. A 114317 (1986).

[62] R. Artuso et al, J. Stat. Phys. 83145 (1996).

[63] S. Kohler, J. Lehmann, P Hänggi, Phys. Rep. 406379 (2005).

[64] T. S. Monteiro et al, Phys. Rev. Lett. 89194102 (2002).

[65] P. H. Jones et al, Preprint physics/0504096 (2005).

[66] W. Acevedo and T. Dittrich, Prog. Theor. Phys. Suppl. 150313 (2003). 
[67] Y. Zhang and S. Sundarajan, Appl. Phys. Lett. 88141903 (2006).

[68] K. M. Frahm and D. L. Shepelyansky, Phys. Rev. Lett. 78 (1440).

[69] M. Leadbeater et al, Phys. Rev. Lett. 811274 (1998).

[70] G. I. Taylor, Proc. Lond. Math. Soc. 20196 (1921).

[71] J. Vollmer, Phys. Rep. 372131 (2002).

[72] N. V. Brilliantov and T. Poeschel, Preprint cond-mat/0506256 (2005).

[73] R. M. Mazo, Brownian Motion, International Series of Monographs of Physics:112, Oxford Science Publications (2002).

[74] S. Redner, A Guide to First-Passage Processes Cambridge University Press, Oxford (2001).

[75] Y. Imry, Introduction to mesoscopic physics, Oxford University Press, Oxford (1997).

[76] C. M. Marcus et al, Phys. Rev. Lett. 69506 (1992).

[77] A. G. Huibers et al, Phys. Rev. Lett. 811917 (1998)

[78] R. de Picciotto et al, Nature 41151 (2001).

[79] D. Weiss et al, Phys. Rev. Lett. 662790 (1991).

[80] D. Weiss et al, Phys. Rev. Lett. 804118 (1993).

[81] R. Fleischmann, T. Geisel, and R. Ketzmerick, Europhys. Lett. 25219 (1994).

[82] B. W. Li and J. Wang, Phys. Rev. Lett., 91044301 (2003).

[83] S. Denisov, J. Klafter, and M. Urbakh, Phys. Rev. Lett. 91194301 (2003).

[84] C. W. Gardiner Handbook of Stochastic Methods, Springer series on Synergetics, Springer (2004).

[85] A. Chen and E. Renshaw, J. Appl. Prob. 29792 (1992).

[86] A. Chen and E. Renshaw, J. Appl. Prob. 31869 (1994).

[87] J. W. Hanneken and D. R. Franceschetti, J. Chem. Phys. 1096533 (1998).

[88] R Klages and N Korabel, J. Phys. A: Math. Gen. 354823 (2002).

[89] G. H. Weiss, Aspects and Applications of the Random Walk, North-Holland, Amsterdam, (2002). 
[90] D. D. Joseph and Luigi Preziosi, Rev. Mod. Phys. 6141 (1989).

[91] D. D. Joseph and Luigi Preziosi, Rev. Mod. Phys. 62375 (1990).

[92] D. Mugnai et al, Phys. Rev. Lett. 68259 (1992).

[93] E. Zauderer, Partial Differential Equations of Applied Mathematics, Wiley-Interscience Series in Pure and Applied Mathematics (1998).

[94] E. P. Wigner, Phys. Rev. 98145 (1955).

[95] F. T. Smith, Phys. Rev 118349 (1960).

[96] J. Heinrichs, J. Phys. : Condens. Matter 21559 (1990).

[97] A. Comtet and C. Texier, J. Phys. A :Math. Gen. 308017 (1997).

[98] C. Texier and A. Comtet, Phys. Rev. Lett 824220 (1999).

[99] C. J. Bolton-Heaton et al, Phys. Rev. B 6010569 (1999)

[100] J. Heinrichs, Phys. Rev. B 65075112 (2002).

[101] S. Kallush, E. Tannenbaum and B. Segev, Chem. Phys. Lett. 396261 (2004).

[102] H. Schomerus et al, Phys. Rev. E63 026605 (2001).

[103] H. Schomerus, Phys. Rev E.64 026606 (2001).

[104] A. Ossipov, T. Kottos and T. Geisel, Phys. Rev. B 6111411 (2000).

[105] A. A. Chabanov and A. Z. Genack, Phys. Rev. Lett 87233903 (2001).

[106] E. Doron, U. Smilansky and A. Frenkel, Phys. Rev. Lett. 653072 (1990).

[107] P. Seba et al, Phys. Rev. E 542438 (1996).

[108] P. W. Brouwer, K. M. Frahm and C. W. J. Beenakker, Phys. Rev. Lett. 784737 (1997).

[109] R. O. Vallejos et al, J. Phys. A :Math. Gen. 314885 (1998).

[110] K. J. H. van Bemmel et al, Physica Scripta T90 278 (2001).

[111] S. K. Joshi, A. K. Gupta and A. M. Jayannavar, Phys. Rev. B 581092 (1998).

[112] D. V. Savin and H. J. Sommers, Phys. Rev. E 68036211 (2003).

[113] J. A. Méndez-Bermúdez and T. Kottos, Phys. Rev. B 72064108 (2005). 
[114] B. Huckestein, R. Ketzmerick and C. H. Lewenkopf, Phys. Rev. Lett. 845504 (2000).

[115] D. Sokolovski and J. N. L. Conor, Phys. Rev A 426512 (1990).

[116] S. A. Ramakrishna and N. Kumar, Phys. Rev. B 613163 (2000).

[117] Y. Fyodorov and H-J. Sommers, Preprint cond-mat/9701037 (1997).

[118] H. van Houten et al, Phys. Rev. B 398556 (1989). 


\title{
Acknowledgments
}

\author{
"Sometimes our light goes out but is blown into flame by another being. Each of us owes deepest \\ thanks to those who have rekindled this light."
}

Dr. Albert Schweitzer

At last the days have come to move on, step by step, path by path but it will be incomplete without thanking those who made it possible.

First I would like to thank Prof. Theo Geisel and "Max-Planck-Institüt für Dynamik und Selbstorganization" (formerly "Max-Planck-Institüt für Strömungsforschung") for providing me with all kinds of necessary support to find a foothold inside the sea of knowledge. Being situated at the University city of Germany where the library has among one of the largest collections of books, it has provided me a wonderful oppurtinity to take another step in my life. Whether you talk about computing facilities, technical papers it have been always easy for me to search through them.

Then to my Guide Dr. Holger Schanz, for helping me take this big leap. He has given me all the freedom and independence to work out using my own crazy ideas. Yet sometimes they fail; but when I approach him, he will be ready to find a way to work them out and help me in every regards. He helped me try find a needle inside the haystack (of knowledge). Whenever there is a problem I used to approach him to discover some solutions, whether it is about the academic details or about papers or even for smaller details. He seems to be always ready to help me out from any kind of situations. He supported me in finding a research grant from Göttingen Graduate School of Physics. All in all I owe him a lot while moving forward in my life. I also thank the Göttingen Graduate School of Physics in providing me the necessary support through the Georg-Christoph-Lichtenberg Stipendium.

Next I would like to thank Regina, Corinna and Katharina for their priceless support while my stay in Göttingen. They made my stay easier. Whenever there is a problem in understanding something written in German or in academics they seems to be always ready to provide some solutions. So also I thank all others in the department for their invaluable support during my stay in Germany.

As far as things are concerned, life has never been easy to me. But I find here and there kindred spirits laying within my friends. I am thankful to them a lot, for not only being here but also in achieving 
pace after pace during all the years in my living. They are the stepping stones to my success. I would like to thank late Digant who was not only a very special friend but something more which I cannot express and his family for their valuable support, even when the things are going against me. Then there are other friends like him, specially Bishu, Bishnu, Biswajeet, Amarjeet and many others for their precious efforts during my hard days. I also thank Ritesh, Srikant from IISc Bangalore; Snigdha didi, Niharika Didi and Bhaina, Narendra, Birabara and Garuda bhai from IIT Bombay for their valuable support to encourage and lend a hand so that I can draw closer to this achievement.

There are few people whom I always remember. Among them are Anuradha Bhabhiji and Siddhartha Bhaia. I remember them for their valuable suggestions, whether I talk about coming to Germany at first instant or coming from Köln and receiving me at the Frankfurt airport or lending out the first hand guide to make my way to Göttingen. I would remember them for their constant inspirations regarding my research and also about small things in my life. I am proud to say that they have been more than friends to me. Thank you Siddhartha Bhai and Anuradha Bhabhiji for all these memories.

Sunit and Surya have facilitated my stay in this small but beautiful city in familiarizing with whereabouts of everyday needs. Especially I thank Sunit for his selfless support and invaluable suggestions. Pratap have been a very good friend as well. We have a nice stay together. He was always with me while roaming around the city and numerous other places. I thank him for recognizing me as I am and helping out in day to day lives. I also thank Krishna, Pradeep, Rekha and Debasmita for alleviating my spirit with small get togethers during my stay in this city.

There are numerous others like Udit, Priya, Harshad, Sunil, Jyoti didi, Hari Bhai to name a few who have made my stay in Germany full of events. I thank them for the numerous visits and events.

It would be incomplete without thanking my Parents and family members who are always been away from me since my childhood. I thank them for their countless patience and never-ending support towards achieving this goal. I owe them a lot who are always eager to hear a word or two, or see me when I come back home.

Last but not least it would be incomplete without thanking my most beautiful friend whom I have dedicated this work, who has been in my heart with me always and all the time and, make me recognize my abilities to try hard and get ahead.

Thanks and Thank you all for everything, 


\section{Erklärung}

1. Ich habe diese Arbeit selbständig und ohne unerlaubte Hilfe angefertigt und dabei nur die angegebenen Quellen benutzt.

2. Ich habe noch keinen Promotionsversuch unternommen.

Göttingen, den : 8. November 2006.

Manamohan Prusty 



\section{Lebenslauf}

\section{Persönlicher Status}

Geburtsdatum : $\quad$ 8. Januar 1980

Geburtsort : $\quad$ Dhenkanal in Orissa, Indien

Staatsangehörigkeit : Indien

\section{Ausbildung}

seit Oktober 2002

Doktorarbeit am Max-Planck-Institut für Dynamik und Selbstorganisation, Göttingen (Betreuer: Dr. Holger Schanz und Prof. Theo Geisel)

2001-2002 Arbeit am Centre for Theoretical Studies, Indian Institute of Science, Bangalore.

1999-2001 Masterarbeit in Physik am Indian Institute of Technology, Mumbai. Projekt: "Hydrogen atom and other Systems in Ellipsoidal Co-ordinates" (Betreuer: Prof. S. H. Patil).

Visiting Students' Research Program am Tata Institute of Fundamental Research, Mumbai. Titel: "Lepton - Nucleon Deep Inelastic Scattering” (Betreuer: Prof. R. S. Bhalerao).

1996-1999 Bachelor in Physik am Ravenshaw (Autonomous) College, Cuttack an der Vanivihar University, Bhubaneswar.

1989-1996

Schulbildung am Navodaya Vidyalaya Rengali (Prüfung durch das Central Board of Secondary Education). 



\section{Scientific Papers Published :}

1. H. Schanz and M. Prusty, "Directed chaos in a billiard chain with transversal magnetic field" in J. Phys. A : Math. and Gen., 38 10085-10100 (2005).

2. M. Prusty and H. Schanz, "Signatures of directed Chaos in the conductance of a nanowire" in Phys. Rev. Lett., 96130601 (2006).

3. M. Prusty and H. Schanz, "Self-pulsing of electron transmission by a transversal magnetic field" in Preprint :: cond-mat/0610799 (submitted to Phys. Rev. Lett.). 